\title{
Asymmetrische Allylierung von prochiralen Ketonen und deren Anwendung in der Synthese von Pinnatolid und Furopinnatin
}

\author{
Dissertation \\ zur Erlangung des Doktorgrades \\ „Doctor rerum naturalium” \\ der Mathematisch-Naturwissenschaftlichen Fakultäten \\ der Georg-August-Universität zu Göttingen
}

vorgelegt von

Thomas Wolfram

aus Sangerhausen

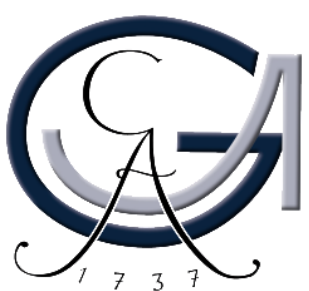

Göttingen 2012 

D7

Referent:

Korreferent:

Tag der mündlichen Prüfung:
Prof. Dr. Dr. h.c. Lutz F. Tietze

Prof. Dr. Ulf Diederichsen

19.04.2012 
Die vorliegende Arbeit wurde in der Zeit von Oktober 2008 bis April 2012 am Institut für Organische und Biomolekulare Chemie der Georg-August-Universität Göttingen unter der Leitung von Prof. Dr. Dr. h.c. Lutz F. Tietze angefertigt.

Mein besonderer Dank gilt Herrn Prof. Dr. Dr. h.c. Lutz F. Tietze für die interessante Themenstellung, sein stetes Interesse am Fortgang dieser Arbeit sowie für die zahlreichen Diskussionen und Anregungen. 
MEINER FAMILIE 



\section{INHALTSVERZEICHNIS}

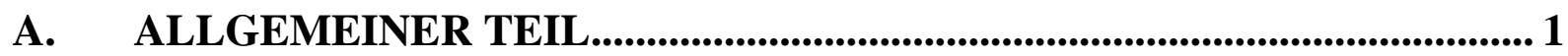

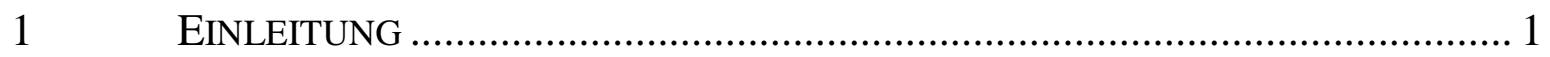

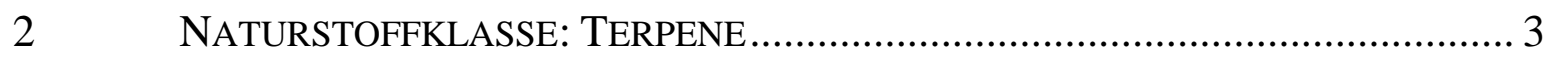

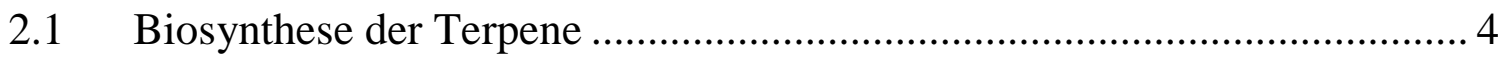

2.1.1 Mevalonsäure-abhängiger Biosyntheseweg....................................... 4

2.1.2 Methylerythritolphosphat-abhängiger Biosyntheseweg ....................... 6

2.1.3 Kopf-Schwanz-Verknüpfung der Isopreneinheiten .............................. 7

2.1.4 Vorkommen und Biosynthese von Furopinnatin und Pinnatolid........... 8

3 ASYMMETRISCHE ALLYLIERUNGEN VON PROCHIRALEN KETONEN ............ 10

3.1 Titankatalysierte Allylierung mit Tetraallylstannan ................................... 11

3.2 Kupferkatalysierte Allylierung mit einem Allylboronsäureester ............... 13

3.3 Silberkatalysierte Allylierung mit Allyltrimethox ysilan .............................. 14

3.4 Chromkatalysierte Allylierung mit Allylbromid ....................................... 15

3.5 Organokatalysierte Allylierung mit Allylboronsäureester .......................... 16

3.6 Anwendungen der asymmetrische Allylierungen von Ketonen in der

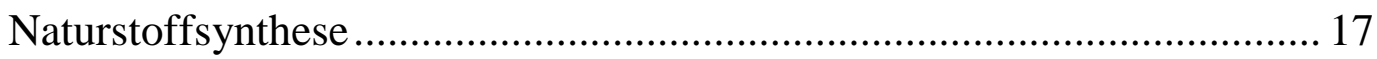

3.6.1 Totalsynthese von (-)-Acutumin ..................................................... 17

4 MULTIKOMPONENTEN-ALLYLIERUNGSREAKTIONEN ................................ 19

4.1 MCAR von Ketonen nach Tietze mit dem NPED-Auxiliar ........................ 20

4.1.1 Totalsynthese des polyoxygenierten Cembrens 59............................ 22

4.2 MCAR von Ketonen nach Tietze mit dem Phenyl-Benzyl-Auxiliar.......... 23

4.2.1 Totalsynthese von (-)-Taurospongin A ........................................... 24

4.2.2 Mechanismus der MCAR nach Tietze …............................................ 25

5 ZIELSETZUNG UND PLANUNG DER ARBEIT ……........................................ 28

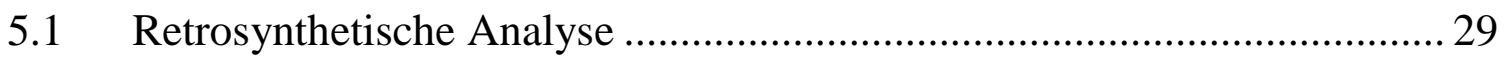

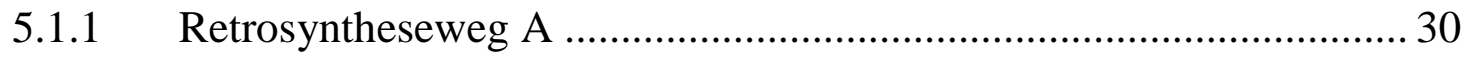

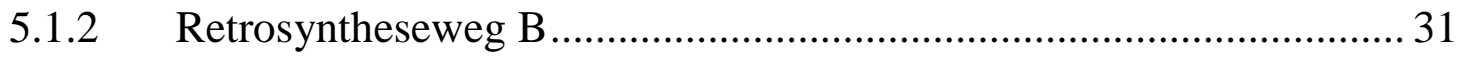

5.1.3 Definition der Teilaufgaben ........................................................... 32

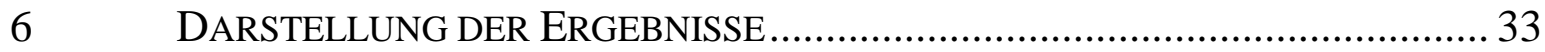




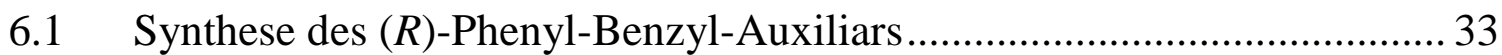

6.2 Syntheseroute A: Allylierung von Sulcaton .............................................. 35

6.3 Syntheseroute B: Allylierung von Lävulinsäuremethylester ……………... 39

6.3.1 Untersuchung der Tietze-Allylierung von prochiralen Ketoestern ..... 39

6.3.2 Diskussion spektroskopischer Daten von Homoallylether 89a .......... 42

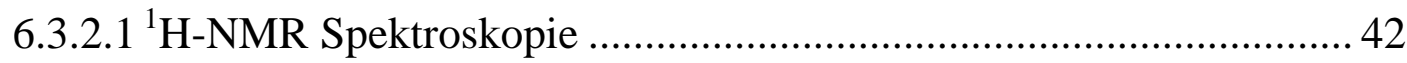

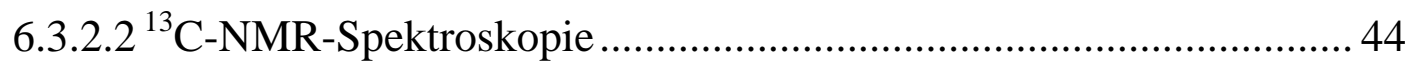

6.3.3 Synthese von Pinnatolid und Furopinnatin ........................................... 46

6.3.4 Abtrennung des anti-Diastereomers durch Umkristallisation des

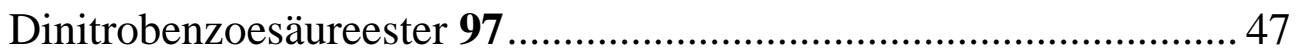

6.3.4.1 Röntgenstrukturanalyse des Dinitrobenzoesäureester 97 .................. 48

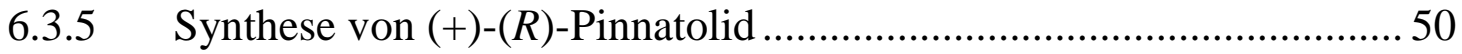

6.3.6 Diskussion spektroskopischer Daten von Pinnatolid........................... 52

6.3.6. ${ }^{1}$ H-NMR-Spektroskopie .................................................................. 52

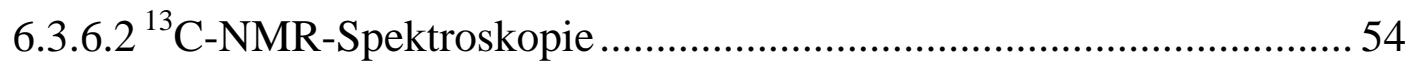

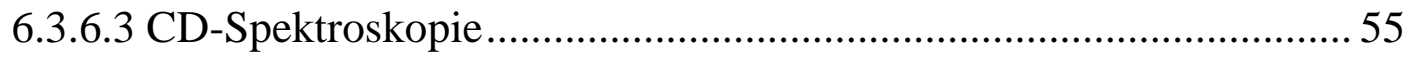

6.3.7 Untersuchungen zur Synthese von $(R)$-Furopinnatin.......................... 57

6.3.8 Diskussion spektroskopischer Daten des Acylfurans 112 .................. 62

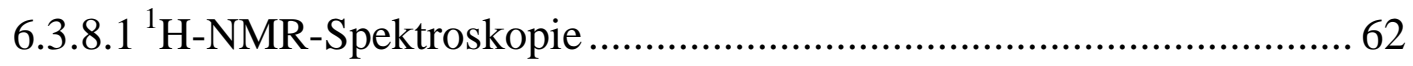

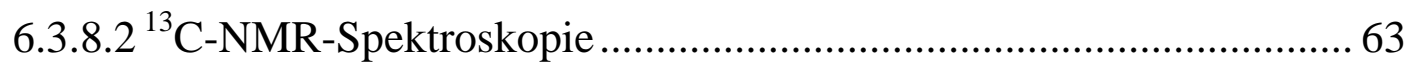

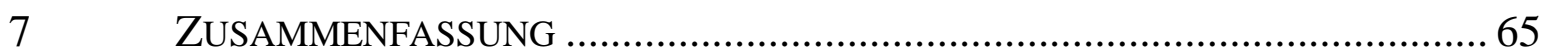

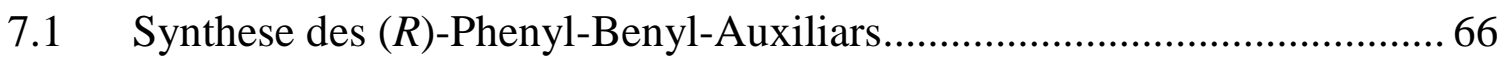

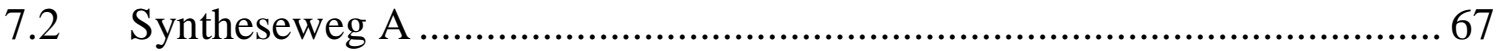

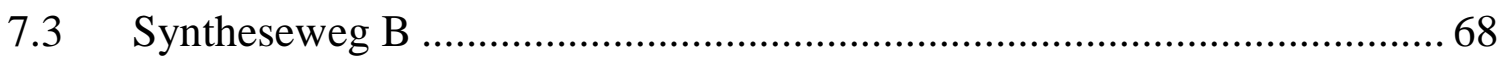

B. EXPERIMENTELLER TEIL ......................................................................... 71

1 ALLGEMEINE ARBEITSVORSCHRIFTEN ……......................................... 71

$1.1 \quad$ Verwendete Geräte ………………………............................................... 71

1.2 Chromatographische Methoden ………………................................ 73

1.3 Allgemeine Reaktionsvorschriften ............................................................ 74

1.3.1 Tietze-Allylierung von prochiralen Methylketonen (AAV1) ............. 74 
1.3.2 Abspaltung der Auxiliargruppe durch Hydrogenolyse (AAV2)........ 74

1.3.3 Abspaltung der Auxiliargruppe unter Birch-Bedingungen (AAV3) .. 74

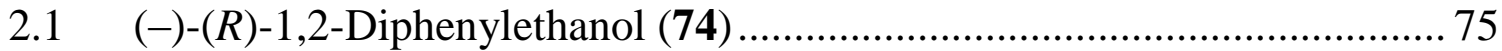

$2.2(+)-(R)-3,5$-Dinitrobenzoesäure(1,2-diphenylethyl)ester (75) .................. 76

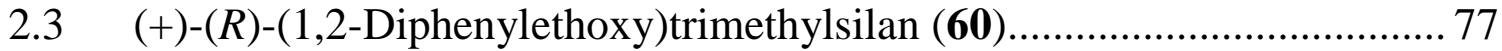

3 SYNTHESE VON (+)-( $R$ )-4,8-DIMETHYLNON-7-EN-4-OLID......................... 79

3.1 (+)-(R,R)-4,8-Dimethyl-4-(1,2-diphenylethoxy)non-1,7-dien (77) .......... 79

$3.2(+)-(R, R)-4,8$-Dimethyl-4-(1,2-diphenylethoxy)non-7-en-1-ol (78) .......... 80

3.3 (+)-(R,R)-4,8-Dimethyl-4-(1,2-diphenylethoxy)non-7-en-1-al (79) ......... 82

3.4 (+)-(R,R)-4,8-Dimethyl-4-(1,2-diphenylethoxy)non-7-ensäure (80) ........ 83

3.5 rac-2-Methyl-2-(4-methylpent-3-enyl)oxiran (83) ................................ 84

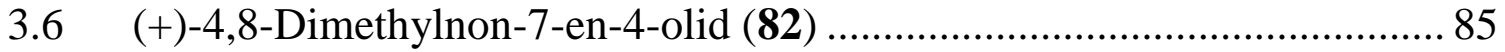

4 TIETZE-ALLYLIERUNG VON KETOESTERN ........................................... 87

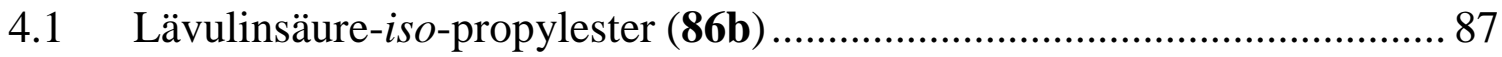

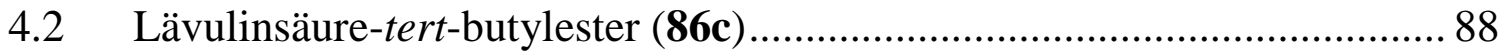

$4.3(+)-(R, R)-4-(1,2-D i p h e n y l e t h o x y)-4-m e t h y l-$ hept-6-ensäuremethylester (89a)

4.4 (+)-(R,R)-4-(1,2-Diphenylethoxy)-4-methyl-hept-6-ensäure-

iso-propylester $(\mathbf{8 9 b})$ 90

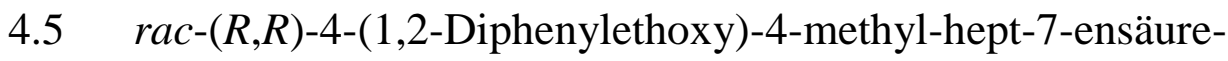
tert-butylester $(\mathbf{8 9 c})$

4.6 rac-(R,R)-5-(1,2-Diphenylethoxy)-5-methyl-oct-7-ensäuremethylester (90)

5 SYNTHESE VON (+)-(R)-PINNATOLID

5.1 (+)-(R,R)-4-(1,2-Diphenylethoxy)-4-methyl-6-oxo-

hexansäuremethylester (95)

5.2 (+)-(R,R)-4-(1,2-Diphenylethoxy)-6-hydroxy-4-methylhexansäuremethylester (96) 95

$5.3(+)-(R, R)-3,5$-Dinitrobenzoesäure-[3-(1,2-diphenylethoxy)6-methoxy-3-methyl-6-oxohexyl]ester (97) 97

5.4 (-)-(R)-5-(2-Hydroxyethyl)-5-methyl-dihydrofuran-2-on (98)................. 98 
5.5 (-)-(R)-5-(2-Hydroxy-4-methyl-pent-3-enyl)-5-methyl-

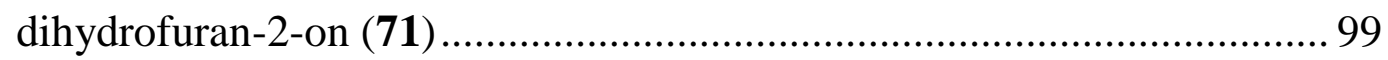

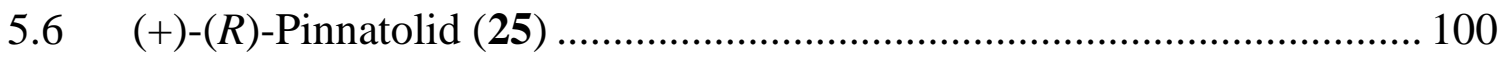

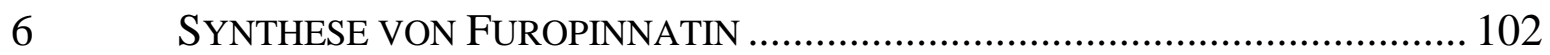

6.1 rac-(R,R)-5-(1,2-Diphenylethoxy)-5-methyloxepan-2-on (102)............. 102

6.2 rac-(R,R)-4-(1,2-Diphenylethoxy)-1-(3-furyl)-6-hydroxy-4-methyl1-hexanon (104)

6.3 rac-(4R,1"R)-4-(1,2-Diphenylethoxy)-

1-(tetrahydrofur-3-yl)6-hydroxy-4-methyl-1-hexanon (105)

6.4 (+)-(5R,1"R)-5-(1,2-Diphenylethoxy)-5-methyl-

7-(2-methylprop-1-enyl)oxepan-2-on (111a+b)

6.5 (-)-(4R,6R,1"R)-4,8-Dimethyl-4-(1,2-diphenylethoxy)-

1-(3-furyl)non-7-en-1-on (112a)

$6.6(+)-(4 R, 6 S, 1 " R)-4,$,8 -Dimethyl-4-(1,2-diphenylethoxy)-

1-(3-furyl)non-7-en-1-on (112b)

C. ANHANG

RÖNTGENSTRUKTURDATEN

ABKÜRZUNGEN UND AKRONYME.

LITERATUR

DANKSAGUNG. 


\section{A. AllGEMEINER TEIL}

\section{$1 \quad$ Einleitung}

Kohlenstoff - Element des Lebens. Aufgrund der Fähigkeit des Kohlenstoffs stabile Ketten aus Einfach- und Mehrfachbindungen mit Elementen, wie z.B. Stickstoff und Sauerstoff, aber vor allem mit sich selbst zu bilden, nimmt der Kohlenstoff eine Sonderstellung im Periodensystem der Elemente (PSE) ein. Des Weiteren ermöglicht die Ausbildung von bis zu vier Bindungen pro Kohlenstoffatom $\mathrm{zu}$ sich selbst oder anderen Elementen eine große strukturelle Diversität, welche sich in der großen Anzahl von bisher hergestellten organischen Verbindungen widerspiegelt.

Gegen Ende der 18. Jahrhunderts stellte J. J. Berzelsius die Theorie auf, dass organische Verbindungen nur in lebenden Organismen gebildet werden können und somit nicht aus anorganischen Stoffen darstellbar sind. Doch bereits 1828 konnte F. Wöhler anhand der Synthese von Harnstoff aus dem anorganischen Ammoniumcyanat die von J. J. Berzelsius aufgestellte Theorie widerlegen. F. Wöhler gilt fortan als Begründer der modernen organischen Chemie.

In Zusammenarbeit mit J. v. Liebig erkannte er, dass selbst Verbindungen mit gleicher Elementaranalyse (also gleicher Summenformel) dennoch verschieden sein können. Dieser Umstand wird Isomerie genannt. Dabei werden verschiedene Formen von Isomerie unterschieden. Wenn die Abfolge der einzelnen Atome einer Struktur (Konstitution) bei zwei Verbindungen gleich ist, spricht man von Konfigurations- bzw. Stereoisomeren. Eine besondere Form von Stereoisomeren sind die Enantiomere, welche sich zueinander wie die Hände eines Menschen verhalten. Obwohl sie vollkommen gleich scheinen, sind sie dennoch nicht identisch. Sie verhalten sich zueinander wie Objekt und Spiegelbild. Dieses Phänomen wird Chiralität (griech.: Händigkeit) genannt und nimmt gerade in Bezug auf Naturstoffe oder andere biologische Substanzen eine wichtige Rolle ein. Interaktionen von chemischen Verbindungen mit Organismen bzw. Lebewesen, wie z.B. die Wirkung von Medikamenten, aber auch bereits einfache Dinge, wie der spezifische Geruch von Substanzen sind an chirale Erkennungsmechanismen gebunden. Dadurch können die beiden Enantiomere einer chiralen Verbindung bei Wechselwirkung mit einem 
Lebewesen verschiedene Wirkungen hervorrufen. Zum Beispiel wird der Geruch des Terpens Limonen (1) abhängig von seiner enantiomeren Form unterschiedlich wahrgenommen. Während das (+)-Limonen, welches in Zitronenöl (65\%) und Orangenöl (>90\%) vorkommt, als ein orangenartiger Geruch empfunden wird, riecht das entgegengesetzte Enantiomer (-)-Limonen, welches vor allem in Nadelhölzern vorkommt, terpentinartig.

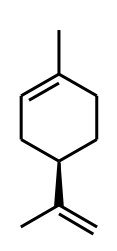

$(+)-1$

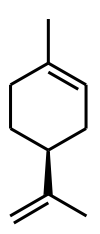

$(-)-1$

Abb. 1-1: Beide enantiomeren Formen von Limonen (1).

Trotz fortschrittlicher analytische Verfahren wie z.B. NMR-Spektroskopie zur Aufklärung der chemischen Struktur von Naturstoffen sowie die fortlaufende Entwicklung neuer Synthesemethoden zum stereoselektiven Aufbau von komplexen organischen Verbindungen gehört die Naturstoffsynthese bis heute zu den anspruchsvollsten Aufgaben der organischen Chemie. 


\section{Naturstoffklasse: Terpene}

Terpene, wie das bereits vorgestellte Limonen (1), sind eine Gruppe von Naturstoffen, welche formal Oligomere des Isoprens (2) sind und sich dennoch durch eine große strukturelle Diversität auszeichnen. Bis heute sind etwa 8.000 Terpene und etwa 30.0000 Terpenoide, wie die funktionalisierten -vorrangig polyoxygenierten- Terpene genannt werden, bekannt. Der Begriff „Terpen“ leitet sich vom Baumharz der Pistacia terebinthus, dem Terpentin, ab und wurde bereits Mitte der 19. Jahrhunderts von A. Kekulé für die im Terpentin enthaltenen Kohlenwasserstoffe geprägt. Ende des 19. Jahrhunderts erweiterte $O$. Wallach im Zusammenhang mit der Erforschung von pflanzlichen etherischen Ölen diese Definition. Die Erkenntnis, dass alle diese Naturstoffe aus sich wiederholenden $\mathrm{C}_{5}$-Einheiten, den Isopreneinheiten, aufgebaut sind und entweder durch eine „Kopf-Schwanz“-, „Kopf-Kopf“- oder „SchwanzSchwanz"-Beziehung (letztere bisher nicht beobachtet) untereinander verknüpft sein können, wurde in den Isoprenregeln festgehalten. ${ }^{[1]}$ Für ihre Leistungen um die Erforschung der Terpene wurden $O$. Wallach und L. Ruzicka jeweils mit dem Nobelpreis für Chemie $(1910,1939)$ geehrt.

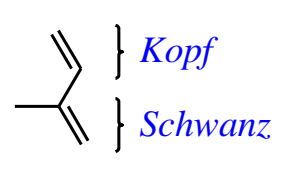

2
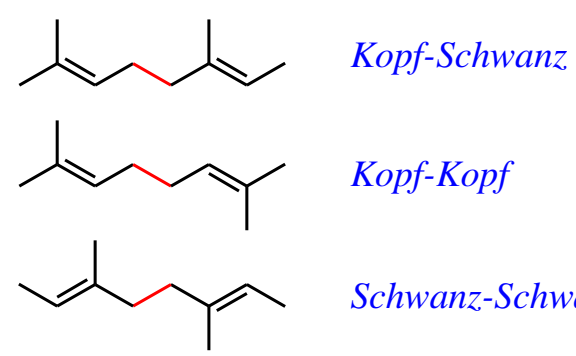

Kopf-Kopf

Abb. 2-1: Darstellung des Kopf-Schwanz-Aufbau von Isopren (2) und die möglichen Verknüpfungsmuster nach den Isoprenregeln ${ }^{[1]}$.

Die Klassifizierung der Terpene erfolgt nach der Anzahl der Isopreneinheiten aus denen sie aufgebaut sind: Hemiterpene $\left(\mathrm{C}_{5}\right)$, Monoterpene $\left(\mathrm{C}_{10}\right)$, Sesquiterpene $\left(\mathrm{C}_{15}\right)$, Diterpene $\left(\mathrm{C}_{20}\right)$, Sesterpene $\left(\mathrm{C}_{25}\right)$, Triterpene $\left(\mathrm{C}_{30}\right)$, usw. Naturstoffe, welche sich von den Terpenen ableiten, aber die Isoprenregeln verletzen (z.B. Steroide) werden ganz allgemein zu den Isoprenoiden gezählt. 


\subsection{Biosynthese der Terpene}

Der Aufbau der Isoprengrundeinheit, dem Isopentenyldiphosphat (IPP, 9) kann über zwei orthogonale Biosynthesewege erfolgen. ${ }^{[2]}$ Im Allgemeinen verwenden die Eukaryoten (Pflanzen, Tiere, Menschen) den Syntheseweg über die Mevalonsäure (MVA, 6) und die Prokaryoten (Bakterien) die Methylerythritolphosphat (MEP, 14)abhängige Route. ${ }^{[3]}$ Abweichend davon verläuft die IPP-Biosynthese von Archaebakterien ausschließlich über den MVA-Syntheseweg und pflanzliche Euzyten können zusätzlich in ihren Plastiden die MEP-Route zur Produktion von IPP nutzen.

\subsubsection{Mevalonsäure-abhängiger Biosyntheseweg}

Zum Aufbau der (R)-Mevalonsäure (MVA, 6) werden zunächst zwei Moleküle AcetylCoA (3) von der entsprechenden Thiolase in einer Claisen-Kondensationsreaktion zu Acetoacetyl-CoA (4) verknüpft. Anschließend wird in einer von der HMG-CoASynthase katalysierten Aldolreaktion eine weitere Acetyl-CoA-Einheit unter Bildung von (S)-Hydroxymethylglutaryl-(HMG)-CoA (5) an 4 addiert. Die durch die HMG-CoA-Reduktase vermittelte Reduktion des Thioesters 5 mit zwei NADPHReduktionsäquivalenten bildet schließlich MVA (6). Im Anschluss erfolgt eine von der MVA- bzw. MVAP-Kinase katalysierte Mono- und Bisphosphorylierung der terminalen Hydroxylgruppe von $\mathbf{6}$ unter Verbrauch von zwei Äquivalenten ATP. Im letzten Schritt der Biosynthese von IPP (9) aus MVA (6) vermittelt die MVAPPDecarboxylase zunächst die in situ Phosphorylierung des tertiären Alkohols von (R)-Mevalonsäure-5-diphosphat (MVAPP, 7) durch eine weitere ATP-Einheit und die anschließende Decarboxylierung der phosphorylierten Zwischenstufe 8 unter Eliminierung von Phosphat (s. Schema 2-1, linke Spalte). 

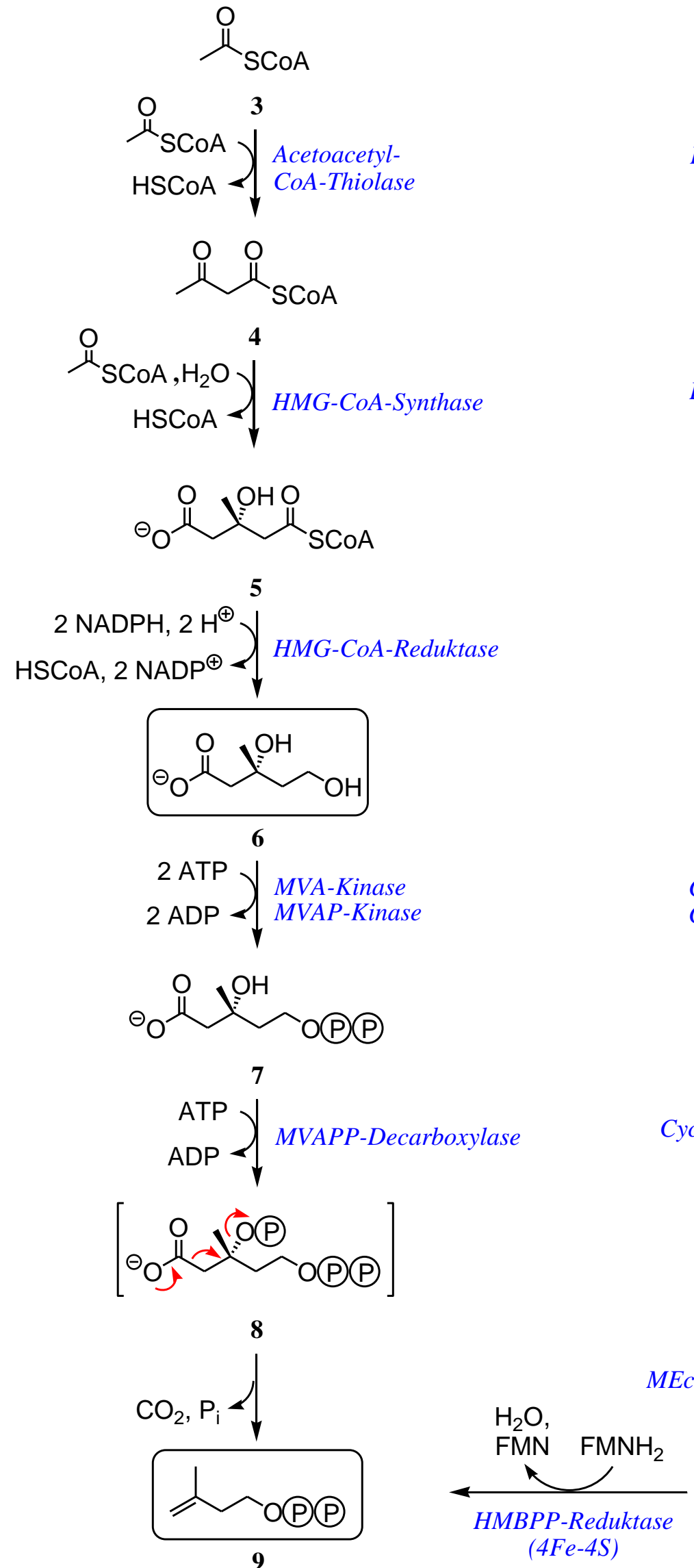

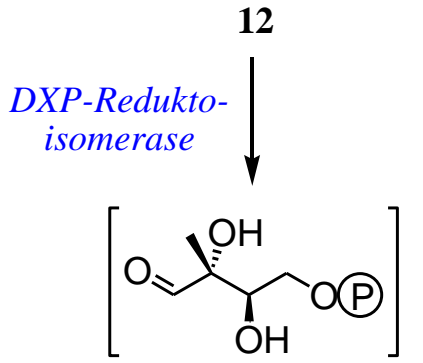

13

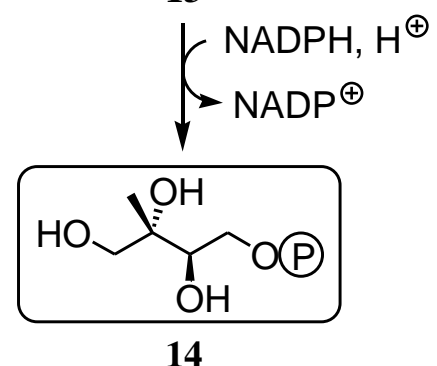

CME-Synthase
CME-Kinase<smiles>CC(CO)(CO)C(O)COC(=O)O</smiles>

15

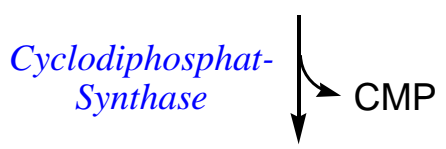<smiles>OCC12COCC(CO1)C2(O)CO</smiles>

16

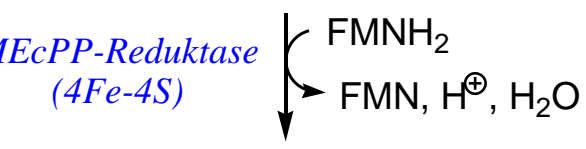<smiles>C/C(=C\CO[18O])CO</smiles>

17

Schema 2-1: Orthogonale Biosynthesewege des Isopentenyldiphosphat (IPP, 9) über Mevalonsäure (MVA, 6) und 2C-Methyl-D-erythritol-4-phosphat (MEP, 14). 


\subsubsection{Methylerythritolphosphat-abhängiger Biosyntheseweg}

Zum Aufbau von 2C-Methyl-D-erythritol-4-phosphat (MEP, 14) werden zunächst (R)-Glycerinaldehyd-3-phosphat (GAP, 10) und Pyruvat (11), welche als Stoffwechselzwischenprodukte (Glykolyse, Glyconeogese) auftreten, in Gegenwart der DXP-Synthase zu 1-Desoxy-D-xylulose-5-phosphat (DXP, 12) in einer durch das Coenzym Thiamindiphosphat (CoT) vermittelten, decarboxylativen Umpolungsreaktion miteinander verknüpft. Die DXP-Reduktoisomerase katalysiert die in situ Isomerisierung von DXP (12) zu 2C-Methyl-D-erythrose-4-phosphat (13) und dessen anschließende Reduktion zu MEP (14) unter Verbrauch eines NADPH-Reduktionsäquivalentes. Für den Mechanismus der Isomerisierung sind sowohl die direkte Umlagerung als auch eine Retroaldol-/Aldolreaktion über eine Endiol-Zwischenstufe denkbar. Zur Überführung von MEP (14) zu IPP (9) wird zunächst durch die CME-Synthase dessen Phosphatgruppe mit einer CTP-Einheit unter Abspaltung von Pyrophosphat verknüpft. Anschließend wird durch die CME-Kinase unter Verbrauch eines weiteren ATP-Moleküls die tertinale Alkoholfunktionalität phosphoryliert. Unter Abspaltung von CMP wird 15 von der Cyclodiphosphat-Synthase zu 2C-MethylD-erythritol-2,4-cyclo-diphosphat (MEcPP, 16) zyklisiert. Die abschließende Transformation von MEcPP (16) zu IPP (9) wird in zwei Schritten über (E)-4-Hydroxy-3-methyl-2-butenyldiphosphat (HMBPP, 17) mit je einer [4Fe-4S]Cluster vermittelten Reduktion erreicht. Die beiden verantwortlichen Enzyme, MEcPP- und HMBPP-Reduktase, ${ }^{[3 \mathrm{c}]}$ auch bekannt als IspG und IspH, benötigen dazu jeweils ein Flavodoxin, ein Flavinmononucleotid (FMN)-enthaltenes Protein, als Reduktionsäquivalent (s. Schema 2-1, rechte Spalte). 


\subsubsection{Kopf-Schwanz-Verknüpfung der Isopreneinheiten}

Für die unter den Terpenen am häufigsten verbreitete Kopf-Schwanz-Verknüpfung werden immer ein IPP (9) und ein Allyldiphosphat mit einander kombiniert. Das einfachste Allyldiphosphat ist 3,3-Dimethylallyldiphosphat (DMAPP, 19), welches in einer reversiblen Isomerisierung von IPP (9) in einem Protonierungs-/Deprotonierungsmechanismus gebildet wird. Die Prenyl-Transferase, GPP-Synthase, verknüpft DMAPP (19) und IPP (9) zum Monoterpen Geranyldiphosphat (GPP, 22). Mechanistisch wird dazu DMAPP (19) unter Abspaltung von Pyrophosphat in das Allylkation 20 überführt, welches nucleophil von IPP (9) angegriffen wird. Deprotonierung des dabei gebildeten tertiären Carbokations 21 liefert schließlich das GPP (22). Der Aufbau des Sesquiterpens Farnesyldiphosphat (FPP, 23) erfolgt in einem analogen Mechanismus ausgehend von GPP (22) unter Kopf-SchwanzVerknüpfung mit einem weiteren Molekül IPP (9). Anhand stereochemischer Untersuchungen mit Isotopenmarkierung (z.B. Deuterium, Tritium) konnte gezeigt werden, dass sowohl bei der Isomerisierung von IPP (9) zu DMAPP (19) als auch bei der Kopf-Schwanz-Verknüpfung durch die Prenyl-Transferasen, jeweils selektiv das pro $R$-Proton abstrahiert wird (s. Schema 2-2). ${ }^{[4]}$
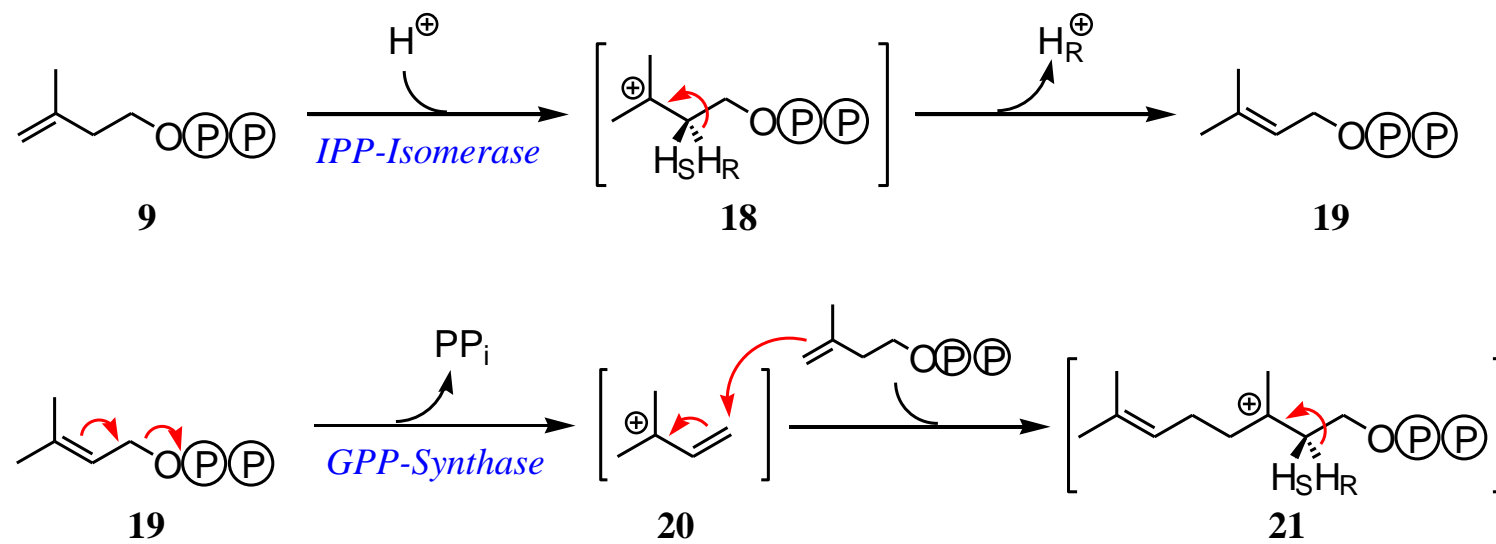

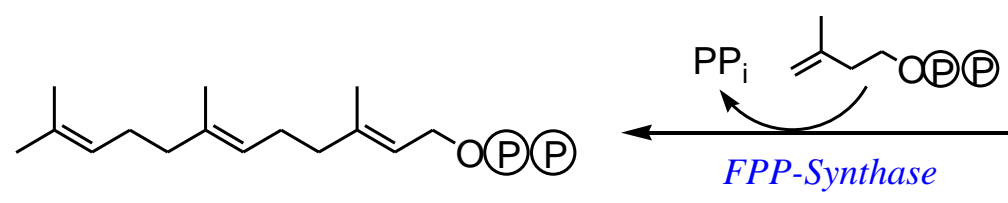

23

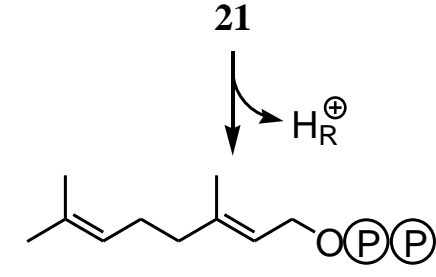

22

Schema 2-2: Isomerisierung von Isoprenyldiphosphat (IPP, 9) zu Dimethylallyldiphosphat (DMAPP, 19) und Aufbau von Gernanyldiphosphat (GPP, 22) und Farnesyldiphosphat (FPP, 23) durch Kopf-Schwanz-Verknüpfung von zwei bzw. drei Isopreneinheiten. 


\subsubsection{Vorkommen und Biosynthese von Furopinnatin und Pinnatolid}

Furopinnatin (24) und Pinnatolid (25) konnten von Bohlmann et al. aus dem namengebenden südafrikanischen Strauch Athanasia pinnata isoliert werden. ${ }^{[5]}$ Beide Verbindungen wurden auch in dem Strauchgewächs Athanasia crithmifolia nachgewiesen. Biologische Untersuchungen hinsichtlich der Wirkungsweise beider Substanzen für die Pfanze oder auf eine potentielle Anwendung als pharmakologische Wirkstoffe wurden bisher nicht durchgeführt. Die Konstitution der chemischen Strukturen von Furopinnatin(24) und Pinnatolid (25) konnten anhand ihrer analytischen Daten (Massenspektrometrie, NMR- und IR-Spektroskopie) aufgeklärt werden. Jedoch die absolute Konfigarition beider Naturstoffe ist bisher nicht bekannt.

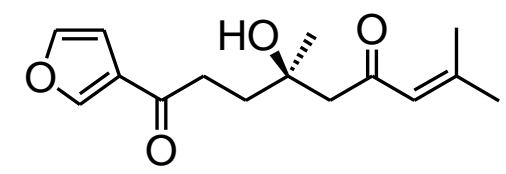

$(R)-24$

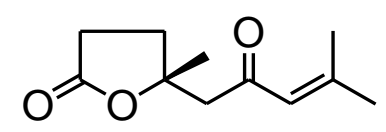

$(R)-25$

Abb. 2-2: $(R)$-Furopinnatin (24) und (R)-Pinnatolid (25).

Das Kohlenstoffgrundgerüst von Furopinnatin (24) setzt sich aus drei Isopreneinheiten zusammen, und gehört somit zu den Sesquiterpenen. Aufgrund des charakteristischen Furanrings findet man häufig auch die Bezeichnung Furosesquiterpen. Hinsichtlich der Biosynthese steht damit ebenfalls fest, dass FPP (23) eine direkte Vorstufe in der Biosynthese von $\mathbf{2 4}$ darstellt. Ein Vergleich beider chemischer Strukturen zeigt an welchen Stellen FPP selektiv oxidiert bzw. hydratisiert werden muss, um es in Furopinnatin (24) zu überführen. Der genaue Biosyntheseweg dieser Oxygenierungsreaktionen wurde bisher nicht aufgeklärt.

Die Struktur von Pinnatolid (25) lässt sich aufgrund seiner elf Kohlenstoffatome weder den Monoterpen $\left(\mathrm{C}_{10}\right)$ noch den Sesquiterpenen $\left(\mathrm{C}_{15}\right)$ zuordnen und wird deswegen zu den Isoprenoiden gezählt. Für die Biosynthese des Pinnatolids (25) vermuten Bohlmann et al., dass es sich um einen partiellen Abbauprozess (möglicherweise eine oxidative Spaltung) des Furopinnatins $\mathbf{2 4}$ handeln könnte. 


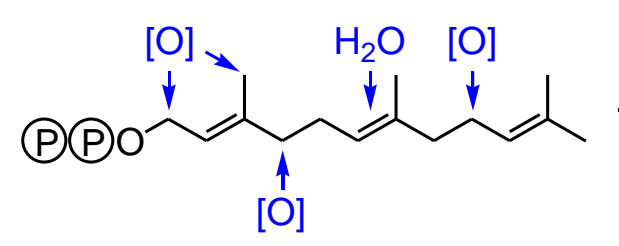

23

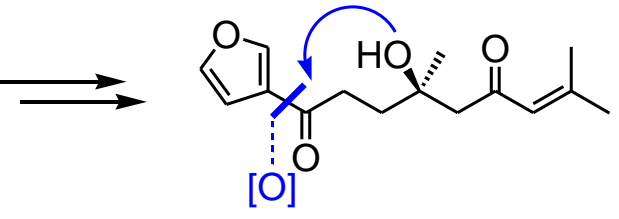

(R)-24

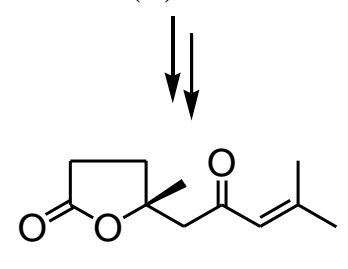

$(R)-25$

Schema 2-3: Biosynthesevorschlag für Furopinnatin (24) und Pinnatolid (25) durch

Polyoxygenierung von FPP (23) und oxidativen Abbauprozess von $\mathbf{2 4}$. 


\section{Asymmetrische Allylierungen von prochiralen Ketonen}

Allylierungen gehören neben der Aldolreaktion zu den am häufigsten eingesetzten asymmetrischen $\mathrm{C}-\mathrm{C}-$ Verknüpfungsreaktionen. Ihre Produkte, die Homoallylalkohole, lassen sich aufgrund der Allylgruppe in vielfältiger Weise transformieren und sind deshalb interessante Intermediate in Synthesen von Naturstoffen oder anderen biologischen aktiven Substanzen. Das Anwendungsspektrum reicht von Additionsreaktionen wie z.B. Epoxidierungen, Hydroborierung oder Wacker-Oxidation über oxidative Spaltungen wie Ozonolyse bis hin zu kettenverlängernden Reaktionen wie z.B. die Metathese.

Brown et al. und Roush et al. waren die Pioniere auf dem Gebiet der asymmetrischen Allylierung von Carbonylverbindungen. Während Brown für die Allylierung von Aldehyden (26) Allylborane, die sich von Terpenen - im Fall von 27 vom $\alpha$-Pinen ableiten, einsetzte, verwendete Roush Allylboronsäureester, wie 29, die sich auf Weinsäurederivate zurückführen lassen (s. Schema 3-1). ${ }^{[7,9]}$<smiles>[R][H]</smiles>

26<smiles>[R]C=O</smiles>

26

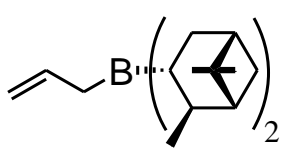

27
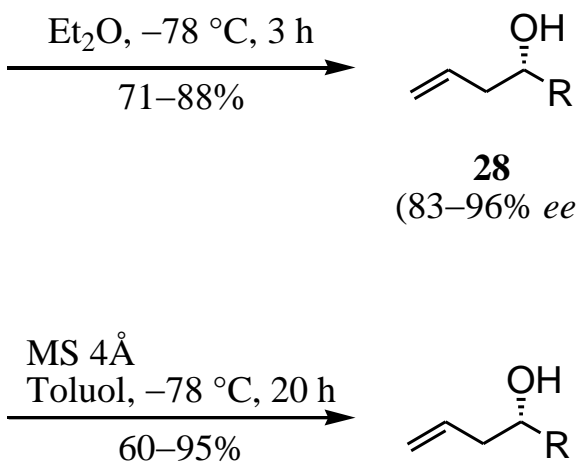

28 $(70-87 \%$ ee $)$

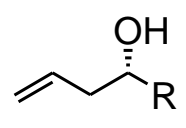

28 $(83-96 \%$ ee $)$

MS $4 \AA$

$\underset{\text { Toluol, }-78^{\circ} \mathrm{C}, 20 \mathrm{~h}}{\longrightarrow}$

29

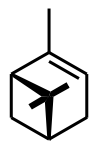

(+)- $\alpha$-Pinen<smiles>O=C([O-])C(O)C(O)C(=O)O</smiles>

(+)-L-Weinsäure

Schema 3-1: $\quad$ Asymmetrische Allylierungen von Aldehyden nach Brown ${ }^{[7]}$ und Roush. ${ }^{[9]}$

Da die Naturstoffe $\alpha$-Pinen und Weinsäure in beiden enantiomeren Formen natürlich vorkommen und somit sowohl $(R)-\mathbf{2 8}$ als auch $(S)$-28 synthetisch leicht zugänglich sind, fanden 27 und 29 in vielfältiger Weise in der Totalsynthese von Naturstoffen Anwendung. ${ }^{[8,10]}$ Der Nachteil beider Verfahren ist zum einen, dass sie für die Umsetzung von Ketonen nicht geeignet sind, und zum anderen die Notwendigleit des Einsatzes von äquimolaren Mengen des chiralen Induktors. 
Im Folgenden werden nun einige katalytische Verfahren zur enantioselektiven Allylierung von prochiralen Ketonen vorgestellt.

\subsection{Titankatalysierte Allylierung mit Tetraallylstannan}

Bereits 1999 stellten Tagliavini et al. das erste katalytische Verfahren zur enantioselektive Allylierung von Ketonen vor. Die Allylierungen von Acetophenon (30f) und Methyl- $n$-hexylketon mit Tetraallylstannan(31) als Allylierungsmittel in Gegenwart von 20 mol\% eines in situ gebildeten Ti(IV)-BINOL-Komplexes ergaben jedoch lediglich Enantiomerenüberschüsse von $65 \%$ bzw. 29\%. ${ }^{[12]}$ Erst die Weiterentwicklungen von Maruoka et al. ${ }^{[14]}$ und Walsh et al. ${ }^{[13]}$ konnten die enantioselektive, titankataylisierte Allylierung von prochiralen Ketonen „hoffähig“ machen.

Durch Untersuchungen der Struktur von Tagliavini's Ti(IV)-BINOL-Katalysatorsystems konnten Walsh et al. zeigen, dass es sich dabei um $\mathrm{Ti}(\mathrm{O} i \text {-Pr })_{2}(\mathrm{BINOL})$ handelt, welches in Lösung als Dimer und im festen Zustand als Trimer vorliegt. Des Weiteren konnte festgestellt werden, dass sich durch Zusatz von Isopropanol die ursprünglich von Tagliavini beobachten Selektivitäten signifikant steigern ließen (s. Tabelle 3-1). ${ }^{[13 a]}$

Tabelle 3-1: Titankatalysierte enantioselektive Allylierung von Ketonen nach Walsh. ${ }^{[13]}$

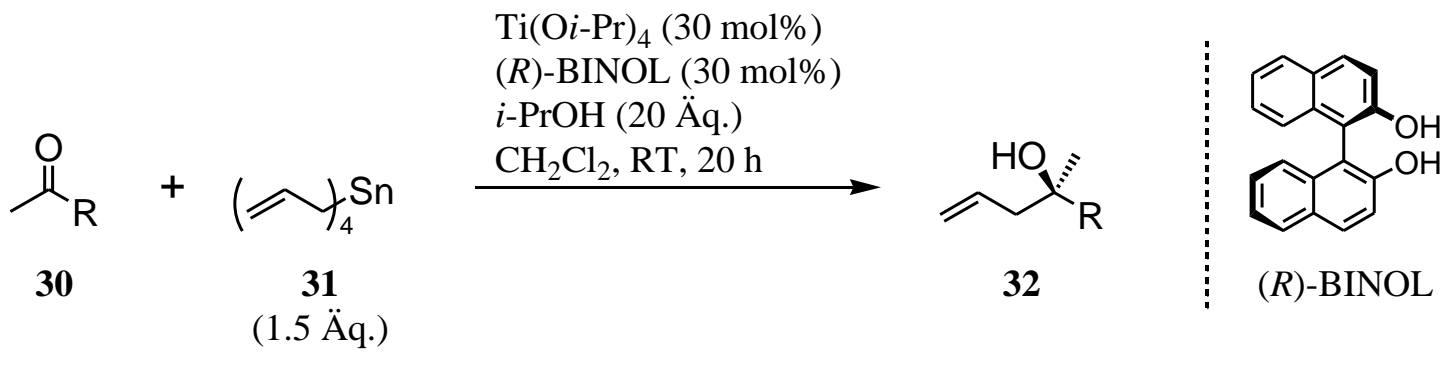

\begin{tabular}{c|c|l|c|c|c}
\hline Nr. & Keton & $\mathbf{R}$ & Homoallylalkohol & Ausbeute [\%] & $\boldsymbol{e}$ [\%] \\
\hline 1 & $\mathbf{3 0 g}$ & $m$-Tol & $\mathbf{3 2 g}$ & 82 & $96^{[\mathrm{a}]}$ \\
\hline & $\mathbf{3 0 h}$ & $4-\mathrm{OMeC}_{6} \mathrm{H}_{4}$ & $\mathbf{3 2 h}$ & 99 & 89 \\
\hline 2 & $\mathbf{3 0 j}$ & $c$-Hex-1-enyl & $\mathbf{3 2 j}$ & 88 & 90 \\
\hline & $\mathbf{3 0 k}$ & $\mathrm{CH}=\mathrm{CHPh}$ & $\mathbf{3 2 k}$ & 99 & 90 \\
\hline 4 & $\mathbf{3 0 c}$ & $\left(\mathrm{CH}_{2}\right) \mathrm{Ph}$ & $\mathbf{3 2 c}$ & 96 & 80 \\
\hline
\end{tabular}

[a] $20 \mathrm{~mol} \% \mathrm{Ti}(\mathrm{O} i \text {-Pr })_{4}$ und $20 \mathrm{~mol}(R)$-BINOL wurden verwendet. 
Mit dieser Allylierungsmethode werden die Homoallylalkohole 32 durchgängig in guten bis sehr guten Ausbeuten erhalten. Bei einer Katalysatorbeladung von 30 mol\% liegt die Enantioselektivität der Reaktion für aromatische und $\alpha, \beta$-ungesättigte Methylketone in einem guten bis sehr guten Bereich. Das aliphatische Methylketon 30c zeigt eine geringfügig schlechtere Selektivität, liegt aber mit immerhin $80 \%$ ee noch in einem respektablen Bereich.

Aufgrund neuerer Untersuchungen von Walsh et al. konnte ebenfalls die benötigte Menge an Katalysator weiter gesenkt werden. Die unter den optimierten Reaktionsbedingungen $\left(10 \mathrm{~mol} \% \mathrm{Ti}(\mathrm{O} i \text {-Pr })_{4}, 20 \mathrm{~mol} \%\right.$ BINOL und 3.0 Äq. Isopropanol, hochkonzentriert ${ }^{[13 c]}$ erhaltenen Selektivitäten weichen aber nur geringfügig von denen in Tabelle 3-1 präsentierten ab.

Mit dem von Maruoka entwickelten Bis-Ti(IV)-BINOL-Katalysatorkomplex (33) lassen sich Aldehyde bereits bei einer Katalysatorbeladung von 3-5 mol\% in guten bis sehr guten Ausbeuten und durchgehend hervorragenden Enantioselektivitäten $(\geq 97 \%$ ee $)$ allylieren. ${ }^{[14 a]}$

Tabelle 3-2: Titankatalysierte enantioselektive Allylierung von Ketonen nach Maruoka. ${ }^{[14]}$

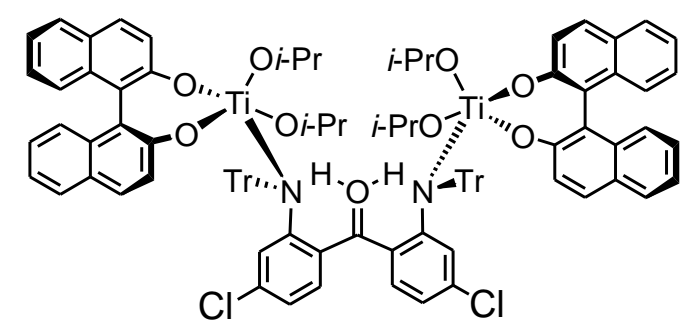

$(S, S)-\mathbf{3 3}$

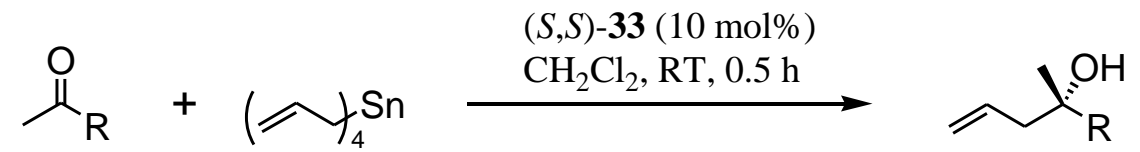

$30 \quad 31$

32

(1.5 Äq.)

\begin{tabular}{c|c|l|c|c|c}
\hline Nr. & Keton & $\mathbf{R}$ & Homoallylalkohol & Ausbeute [\%] & $\boldsymbol{e}$ [\%] \\
\hline 1 & $\mathbf{3 0 f}$ & $\mathrm{Ph}$ & $\mathbf{3 2 f}$ & 96 & 71 \\
\hline 2 & $\mathbf{3 0 h}$ & $4-\mathrm{OMeC}_{6} \mathrm{H}_{4}$ & $\mathbf{3 2 h}$ & 95 & 60 \\
\hline 3 & $\mathbf{3 0 i}$ & Naphthyl & $\mathbf{3 2 i}$ & 99 & 75 \\
\hline 4 & $\mathbf{3 0 k}$ & $\mathrm{CH}=\mathrm{CHPh}$ & $\mathbf{3 2 k}$ & 98 & 54 \\
\hline 5 & $\mathbf{3 0 c}$ & $\left(\mathrm{CH}_{2}\right)_{2} \mathrm{Ph}$ & $\mathbf{3 2 c}$ & 99 & 52 \\
\hline
\end{tabular}


Trotz Erhöhung der Katalysatormenge auf 10 mol\% konnte diese Methode jedoch nur bedingt auf die Allylierung von Ketonen übertragen werden (s. Tabelle 3-2). ${ }^{[14 b]}$ Obwohl unter den genannten Reaktionsbedingungen die Allylierungsprodukte 32 in nahezu quantitativen Ausbeuten erhalten werden, sind bei Einsatz von 10 mol\% des Katalysators $\mathbf{3 3}$ die Enantioselektivitäten für aromatische Ketone lediglich moderat und für $\alpha, \beta$-ungesättigte sowie aliphatische Ketone nur mäßig. Erst nach einer weiteren Erhöhung der Katalysatormenge auf $30 \mathrm{~mol} \%$ konnte für die Allylierung von Acetophenon (30f) eine gute Selektivität von $92 \%$ ee erreicht werden.

\subsection{Kupferkatalysierte Allylierung mit einem Allylboronsäureester}

Die kupferkatalysierte aysmmetrische Allylborierung von Ketonen wurde von Shibasaki et al. entwickelt. Der eigentliche Katalysator $\mathrm{CuF}-(R, R)-i$-Pr-DuPHOS wird dabei in situ aus Kupfer(II)-flourid und dem chiralen Liganden $(R, R)-i$-Pr-DuPHOS (35) im Verhältnis 1:2 gebildet. Die Zugabe von katalytischen Mengen an Lanthantriisopropoxid führt zu einer Beschleunigung der Reaktion und die Reaktionszeiten verkürzen sich von zuvor $20 \mathrm{~h}$ auf $1 \mathrm{~h}$ (s. Tabelle 3-3). ${ }^{[11]}$

Tabelle 3-3: Kupferkatalysierte enantioselektive Allylierung von Ketonen nach Shibasaki. ${ }^{[1]}$

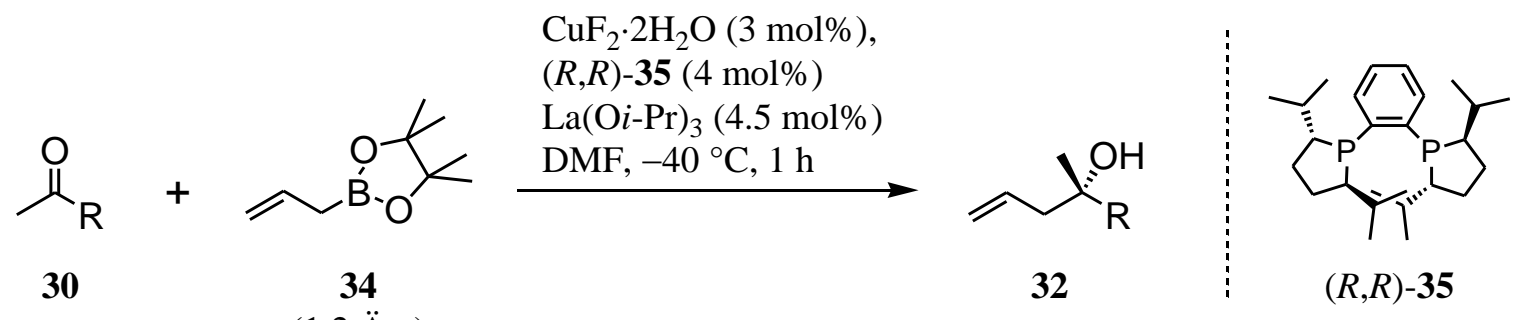

(1.2 Äq.)

\begin{tabular}{c|c|l|c|c|c}
\hline Nr. & Keton & $\mathbf{R}$ & Homoallylalkohol & Ausbeute [\%] & $\boldsymbol{e e ~ [ \% ] ~}$ \\
\hline 1 & $\mathbf{3 0 f}$ & $\mathrm{Ph}$ & $\mathbf{3 2 f}$ & 94 & 82 \\
\hline 2 & $\mathbf{3 0 g}$ & $m$-Tol & $\mathbf{3 2 g}$ & 83 & 83 \\
\hline 3 & $\mathbf{3 0 j}$ & $c$-Hex-1-enyl & $\mathbf{3 2} \mathbf{j}$ & 87 & 90 \\
\hline 4 & $\mathbf{3 0 c}$ & $\left(\mathrm{CH}_{2}\right) \mathrm{Ph}$ & $\mathbf{3 2 c}$ & 96 & 67 \\
\hline 5 & $\mathbf{3 0 e}$ & $t-\mathrm{Bu}$ & $\mathbf{3 2 e}$ & 99 & 91 \\
\hline
\end{tabular}


Während die Ausbeuten dieser Reaktion durchgängig im guten bis sehr guten Bereich liegen, variieren die Enantioselektivitäten abhängig vom elektronischen Charakter der Ketone. Aromatische und $\alpha, \beta$-ungesättigte Ketone lassen sich in guten bis sehr guten Enantioselektivitäten allylieren. Bei den aliphatischen Ketonen hingegen werden nur bei sterisch sehr anspruchsvollen Alkylresten, wie Tertbutyl, gute Selektivitäten erhalten. Aber gerade in Bezug auf $\alpha$-unverzweigte Ketone, wie 30c, liegt die Selektivität mit $67 \%$ ee lediglich in einem moderaten Bereich.

\subsection{Silberkatalysierte Allylierung mit Allyltrimethoxysilan}

Die silberkatalysierte asymmetrischen Allylierung mit Allyltrimethoxysilan (36) und den achsenchiralen Liganden 37 wurde von Yamamoto et al. enwickelt und eignet sich besonders für cyclische aromatische Ketone (s. Tabelle 3-4). ${ }^{[16]}$

Tabelle 3-4: Silberkatalysierte enantioselektive Allylierung von Ketonen nach Yamamoto. ${ }^{[16]}$

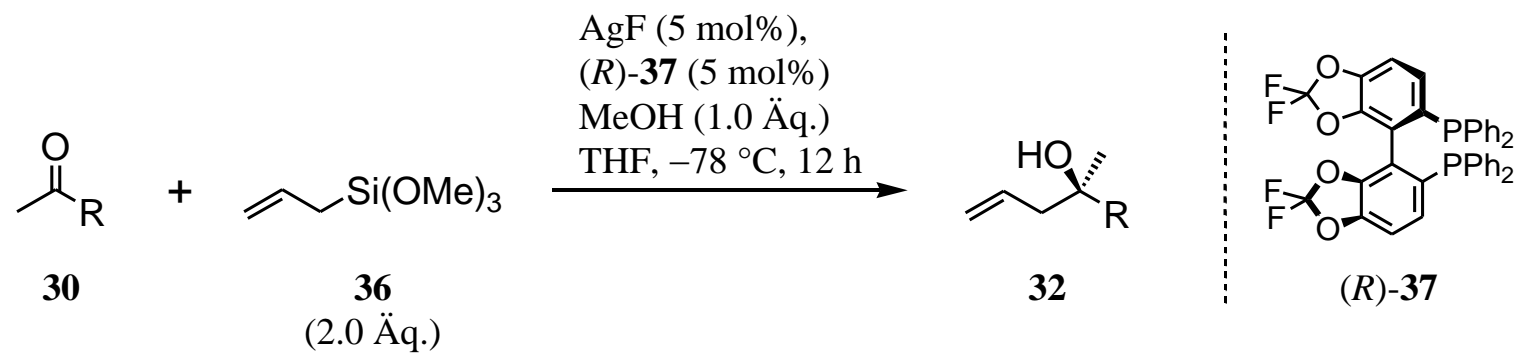

\begin{tabular}{c|c|l|c|c|c}
\hline Nr. & Keton & $\mathbf{R}$ & Homoallylalkohol & Ausbeute [\%] & $\boldsymbol{e e ~ [ \% ] ~}$ \\
\hline 1 & $\mathbf{3 0 f}$ & $\mathrm{Ph}$ & $\mathbf{3 2 f}$ & 96 & 82 \\
\hline 2 & $\mathbf{3 0 h}$ & $4-\mathrm{OMeC}_{6} \mathrm{H}_{4}$ & $\mathbf{3 2 h}$ & 63 & 78 \\
\hline 3 & $\mathbf{3 0 i}$ & Naphthyl & $\mathbf{3 2 i}$ & 98 & 95 \\
\hline 4 & $\mathbf{3 0 j}$ & $c-\mathrm{Hex}-1-$-enyl & $\mathbf{3 2 j}$ & 56 & 95 \\
\hline 5 & $\mathbf{3 0 c}$ & $\left(\mathrm{CH}_{2}\right)_{2} \mathrm{Ph}$ & $\mathbf{3 2 c}$ & 42 & 65 \\
\hline
\end{tabular}

Generell liegen die Ausbeuten und Enantioselektivitäten für aromatische Ketone in einem guten bis sehr guten Bereich. Aliphatische Ketone, wie 30c, sind unter den Reaktionsbedingungen von Yamamoto tendenziell schlechte Substrate und geben lediglich mäßige Ausbeuten und Enantioselektivitäten. 


\subsection{Chromkatalysierte Allylierung mit Allylbromid}

Die von Sigman et al. entwickelte Chrom(II)-katalysiete Allylierung von Ketonen gehört zu den Nozaki-Hiyama-Kishi-(NHK)-Reaktion, ${ }^{[17]}$ welche ursprünglich für die Addition von Vinyl- und Allylresten an Aldehyde entwickelt worden ist. Die katalytische Variante der NHK-Reaktion wurde von Fürstner et al. entwickelt. ${ }^{[18]}$ Dabei dient elementares Mangan als Reduktionsmittel und TMSCl ist aufgrund der intermediär gebildeten, stark substitutionsinerten $\mathrm{Cr}$ (III)-Komplexe erforderlich, um die Allylierungsprodukte aus diesen herauszulösen. Ohne hydrolytische Aufarbeitung mit ges. $\mathrm{NaHCO}_{3}$-Lsg. werden die entsprechenden Trimethylsilylether der Homoallylalkohole 32 erhalten.

Sigman et al. konnte unter Verwendung eines leicht modifizierbaren Aminosäureansatz zum Design von chiralen Liganden das Derivat des Dipeptids L-Pro-D-Val 39 als potenten Kandidaten für die asymmetrische Allylierung von Ketonen identifizieren (s. Tabelle 3-5).

Tabelle 3-5: Chromkatalysierte enantioselektive Allylierung von Ketonen nach Sigman. ${ }^{[19]}$

i) $\mathrm{CrCl}_{3}(10 \mathrm{~mol} \%), \mathrm{Mn}(0)(2.0$ Äq.), $39(10 \mathrm{~mol} \%), \mathrm{Et}_{3} \mathrm{~N}(20 \mathrm{~mol} \%)$, TMSCl (4.0 Äq.), THF, $0{ }^{\circ} \mathrm{C}, 24 \mathrm{~h}$

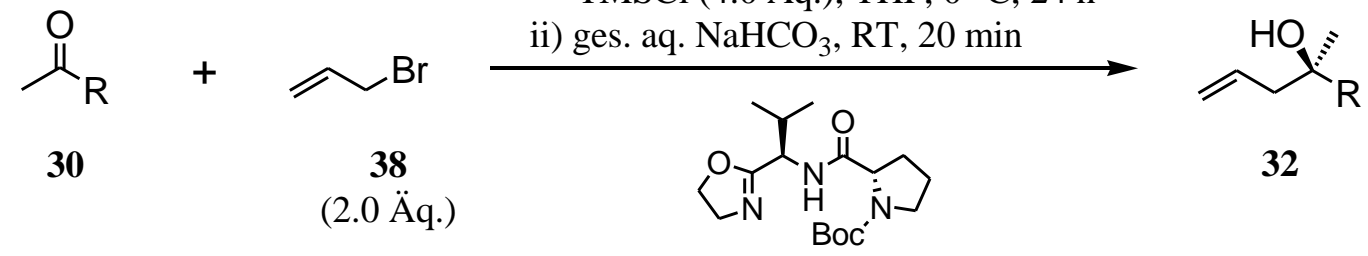

39

\begin{tabular}{c|c|l|c|c|c}
\hline Nr. & Keton & $\mathbf{R}$ & Homoallylalkohol & Ausbeute [\%] & ee [\%] \\
\hline 1 & 30f & Ph & 32f & 82 & 92 \\
\hline 2 & 30i & Naphthyl & 32i & 95 & 92 \\
\hline 3 & 30c & $\left(\mathrm{CH}_{2}\right)_{2} \mathrm{Ph}$ & 32c & 93 & 33 \\
\hline
\end{tabular}

Während aromatische Ketone sich in guten bis sehr guten Ausbeuten und Enantioselektivitäten allylieren lassen, versagt diese Methode hinsichtlich der Selektivität der Allylierung von aliphatischen, $\alpha$-unverzweigte Ketone, wie 30c, völlig. 


\subsection{Organokatalysierte Allylierung mit Allylboronsäureester}

Eine hervorragende Methode zur asymmetrischen Allyllierung von aromatischen Ketonen ist die von Schaus et al. entwickelte, organakatalysierte Allylborierung. Als Katalysator dient das BINOL-Derivat (S)-41, welches anfänglich noch mit einer Katalysatorbeladung von $15 \mathrm{~mol} \%$ bei $-35^{\circ} \mathrm{C}$ eingesetzt wurde. ${ }^{[20 a]}$ Anhand von Untersuchungen in Anlehnung an Walsh bei der titalkatalysierten Allylierung erkannte die Gruppe um Schaus ebenfalls einen direkten Zusammenhang zwischen der Enantioselektivität, der Katalysatorbeladung und der Menge an zusätzlich zugegebenen Alkohol. ${ }^{[20 b]}$

Tabelle 3-6: Organokatalysierte enantioselektive Allylierung von Ketonen nach Schaus. ${ }^{[20 \mathrm{~b}]}$

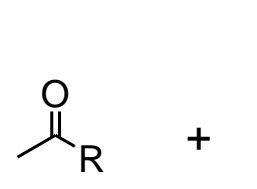

30

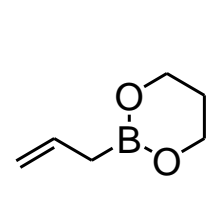

40

(1.5 Äq.)
(S)-41 (2 mol\%), $t$ - $\mathrm{BuOH}(2.0$ Äq.) $\stackrel{\text { Toluol, RT, } 24 \mathrm{~h}}{\longrightarrow}$<smiles>[R]C(C)(O)CC=C</smiles>

32

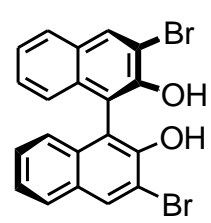

(S)-41

\begin{tabular}{c|c|l|c|c|c}
\hline Nr. & Keton & $\mathbf{R}$ & Homoallylalkohol & Ausbeute [\%] & ee [\%] \\
\hline 1 & $\mathbf{3 0 f}$ & $\mathrm{Ph}$ & $\mathbf{3 2 f}$ & 96 & 98 \\
\hline 2 & $\mathbf{3 0 h}$ & $4-\mathrm{OMeC}_{6} \mathrm{H}_{4}$ & $\mathbf{3 2 h}$ & 88 & 98 \\
\hline 3 & $\mathbf{3 0 j}$ & $c$-Hex-1-enyl & $\mathbf{3 2 j}$ & 96 & 96 \\
\hline
\end{tabular}

Unter den optimierten Reaktionsbedingungen (s. Tabelle 3-6) konnten selbst bei Umgebungstemperatur und lediglich $2 \mathrm{~mol} \%$ Katalysatorbeladung für aromatische Ketone wie 30f und 30h sowie für das zyklische Enon 30j durchgehend sehr gute Ausbeuten und exzellente Enantioselektivitäten ( $\geq 96 \%$ ee) erreicht werden. 


\subsection{Anwendungen der asymmetrische Allylierungen von Ketonen in der Naturstoffsynthese}

Da für die asymmetrische Allylierung von Aldehyden eine Vielzahl von stöchiometrischen (z.B. Brown) aber auch katalytischen Methoden (z.B. Maruoka) mit breiten Substratspekten zur Verfügung stehen, haben diese für den stereoselektiven Aufbau von sekundären Alkoholen vielfach Verwendung in Totalsynthesen von Naturstoffen gefunden. ${ }^{[8,15]}$ Hingegen wurden die großen Fortschritte auf dem Gebiet der asymmetrischen Allylierung von Ketonen erst etwa in den letzten 10 Jahren gemacht, wodurch der Einzug dieser Verfahren in die Totalsynthese von Naturstoffen bisher noch weitesgehend austeht.

\subsubsection{Totalsynthese von (-)-Acutumin}

Zum stereoselektiven Aufbau eines der quartären Stereozentrumen von (-)-Acutumin (46) strebten Castle et al. eine sehr elegante Kombination aus asymmetrischer Allylierung eines Enons und anschließender anionischer Oxy-Cope-Umlagerung an. Jedoch stellte sich in einer früheren Untersuchung von Castle et al. heraus, dass die katalytischen Methoden von Walsh, Shibasaki, Yamamoto, Sigman und Schaus bei der Allylierung von derartigen Enone, wie 42, entweder keinen Umsatz oder das Allylierungsprodukt lediglich in racemischer Form lieferten. Erst durch Verwendung des Nakamura-Reagenzes (43), welches ursprünglich von Nakamura et al. für die asymmetrische Allylierung von Alkinylketonen eingesetzt wurden war, ${ }^{[21]}$ gelang die asymmetrische Allylierung von Ketonen, wie 42. ${ }^{[23 a]}$ Anhand dieser Ergebnisse konnte der Homoallylalkohol 44 unter Verwendung von $(S, S)-43$ mit einer guten Ausbeute von $76 \%$ und einer Diastereoselektivität von 93:7 erhalten werden. Der Naturstoff (-)-46 war nach der von Kalium-tert-butoxid und einem Kronenether vermittelten anionischen Oxy-Cope-Umlagerung in sieben weiteren Schritten zugänglich. ${ }^{\text {[23b] }}$ 


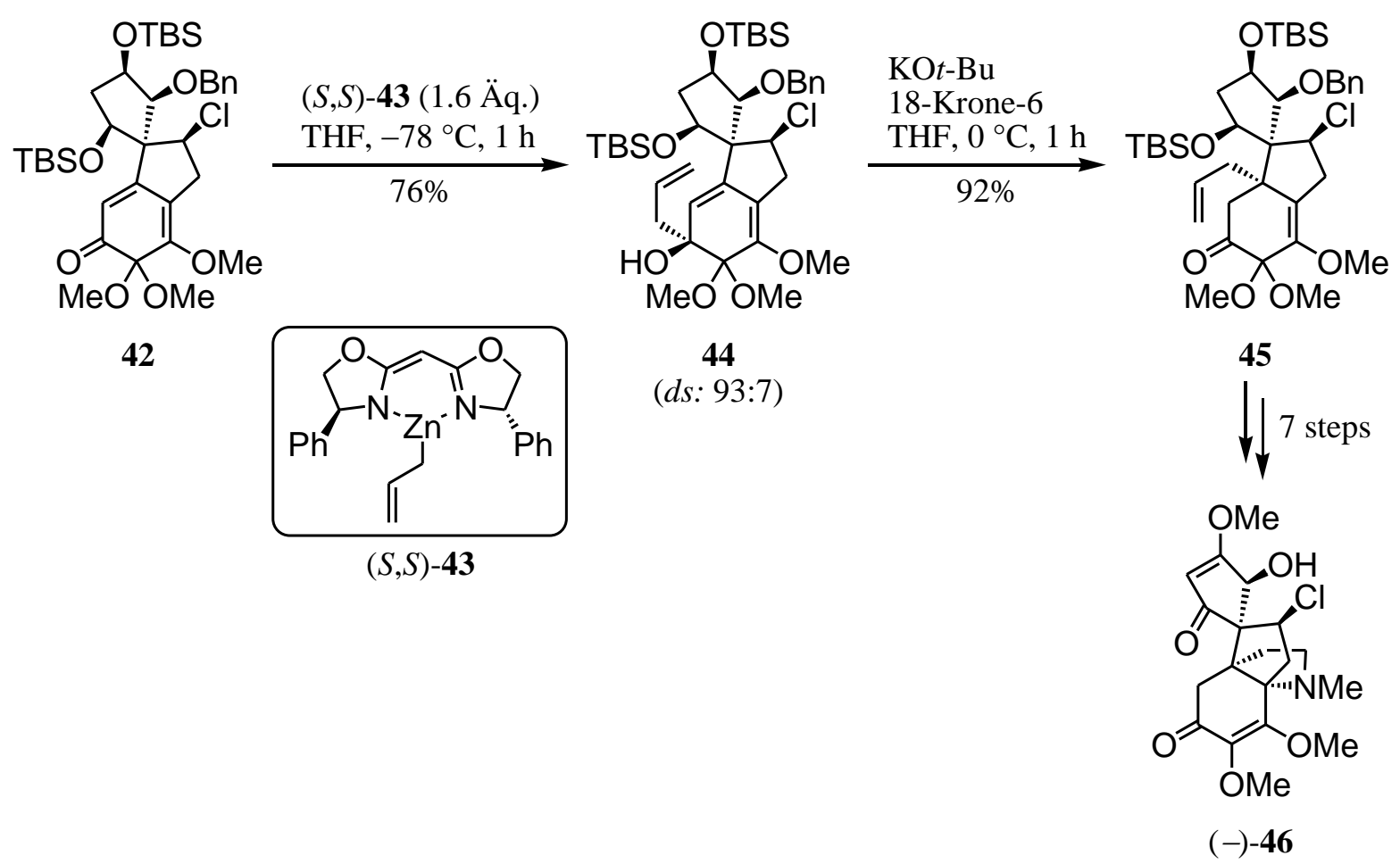

Schema 3-2: $\quad$ Ausschnitt aus der Totalsynthese von (-)-Acutumin (46) nach Castle. ${ }^{[23 b]}$ 


\section{Multikomponenten-Allylierungsreaktionen}

Die meisten in Kapitel 3 vorgestellen asymmetrischen Allylierungsmethoden erreichten bei der Allylierung von aliphatischen, $\alpha$-unverzweigten Ketonen lediglich mäßige Enantioselektiven und scheiterten somit an der schwierigen, facialen Differenzierung zwischen zwei unverzweigten Kohlenstoffresten an der Ketogruppe. Aus diesem Grund soll im Folgenden eine weitere Art von Allylierungsreaktion vorgestellt werden: die Multikomponent-Allylierungsreaktion (MCAR).

Bei dieser säurekatalytischen asymmetrischen Allylierungen mit untoxischem Allyltrimethylsilan (47) als Allylierungsmittel handelt es sich um eine modifizierte Sakurai-Hosomi-Reaktionen. ${ }^{[24]}$ Der Begriff Multikomponenten-Allylierungsreaktion kommt dadurch zustande, dass neben der zu allylierenden Carbonylverbindung und dem Allylierungsmittel 47 eine weitere Komponente zum Einsatz kommt: ein chiraler Silylether (48), welcher in der Reaktion als Auxiliar fungiert. Als Katalysatoren können sowohl Lewis-Säuren, wie z.B. $\mathrm{Ph}_{2} \mathrm{BOTf} \mathrm{TrClO}_{4},{ }^{[25 \mathrm{a}]}$ TMSOTf, ${ }^{[25 \mathrm{~b}, 26]}$ aber auch starke Brønsted-Säuren wie TfOH ${ }^{[28,33]}$ dienen.

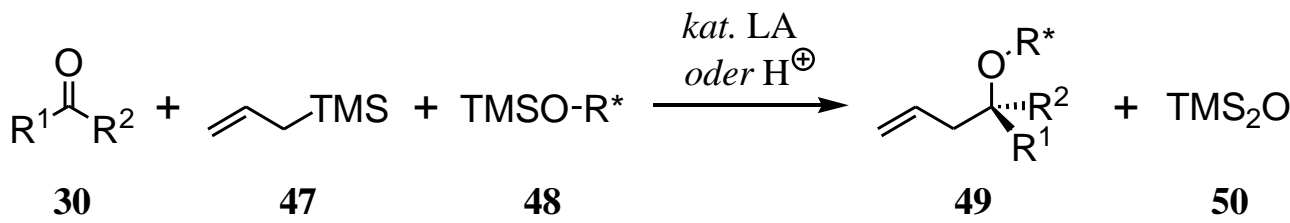

Schema 4-1: Allgemeine Reaktionsgleichung einer Multikomponenten-Allylierungsreaktion.

Während Mukaiyama et al.und Markó et al. für die asymmetrische Allylierung von Aldehyden das vom Phenylmethylcarbinol abgeleitete Auxiliar 51 und Rychnovsky et al.von Arylsilylcarbinolen abgeleitete Auxiliare, wie $\mathbf{5 2}$ verwendeten, setzten Tietze et al. zunächst das Norpseudoephedrin-Derivat 53 ein. Bei Verwendung von $\mathbf{5 2}$ kann anschließend durch Behandlung des Allylierungsproduktes mit einer Fluoridquelle, wie z.B. TBAF, die Auxiliargruppe auch in eine gewöhnliche Benzylschutzgruppe überführt werden. 
<smiles>COC(C)c1ccccc1</smiles>

$(S)-51$<smiles>CC[SiH2]C(OC)c1ccccc1</smiles>

$(S)-52$<smiles>COC(c1ccccc1)C(C)N[I+]C(F)(F)F</smiles>

$(S, S)-53$

Abb. 4-1: Auxiliare der asymmetrischen MCAR von Aldehyden nach Mukaiyama, Markó (51), ${ }^{[25]}$ Rychnovsky (52) ${ }^{[26]}$ und Tietze (53). ${ }^{[27]}$

\subsection{MCAR von Ketonen nach Tietze mit dem NPED-Auxiliar}

Die besten Selektivitäten bei der asymmetrischen Allylierung von Aldehyden wurden von Tietze et al. unter Verwendung des Norpseudoephedrin-Derivat (NPED, 53) erzielt. ${ }^{[27]}$ Auch die Allylierung von prochiralen Ketonen, wie beispielsweise Butanon (30a), an dem viele andere Allylierungsmethoden wegen der schwierigen faciale Differenzierung zwischen einem Methyl- und Ethylrest hinsichtlich der Selektivität scheiterten, gelang mit 90\% Ausbeute und in einer bemerkenswerten Diastereoselektivität von $90: 10{ }^{[28]}$

Tabelle 4-1: Ergebnisse der Allylierung nach Tietze mit dem NPED-Auxiliar (53).

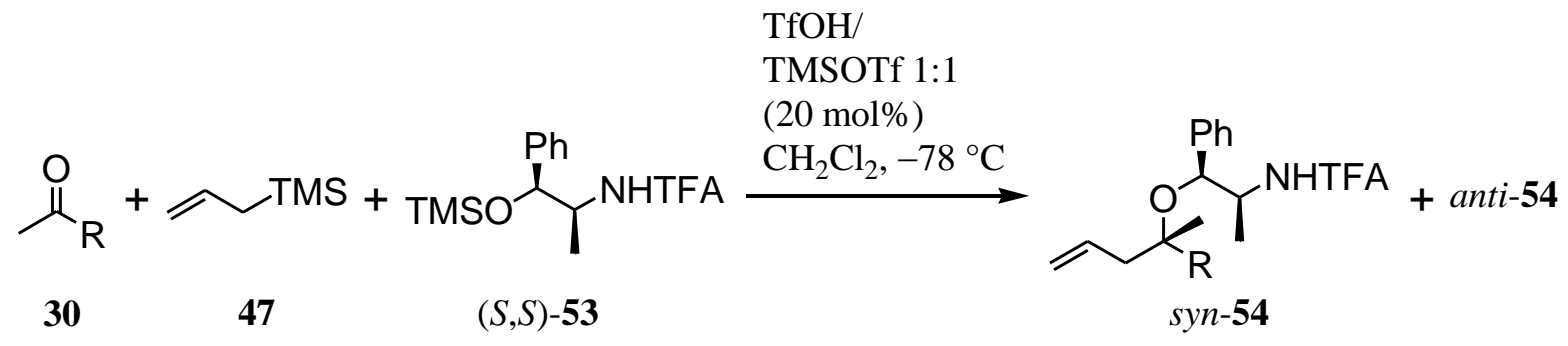

\begin{tabular}{c|c|l|c|c|c}
\hline Nr. & Keton & $\mathbf{R}$ & Homoallylether & Ausbeute [\%] & syn:anti \\
\hline 1 & $\mathbf{3 0 a}$ & Et & $\mathbf{5 4 a}$ & 87 & $90: 10$ \\
\hline 2 & $\mathbf{3 0 b}$ & Pent & $\mathbf{5 4 b}$ & 79 & $88: 12$ \\
\hline 3 & $\mathbf{3 0 c}$ & $\left(\mathrm{CH}_{2}\right)_{2} \mathrm{Ph}$ & $\mathbf{5 4 c}$ & 63 & $91: 9$ \\
\hline 4 & $\mathbf{3 0 d}$ & $i-\mathrm{Pr}$ & $\mathbf{5 4 d}$ & 59 & $96: 4$ \\
\hline 5 & $\mathbf{3 0 e}$ & $t-\mathrm{Bu}$ & $\mathbf{5 4}$ & 22 & $91: 9$ \\
\hline
\end{tabular}

Die Reaktion mit $\alpha$-unverzweigten, aliphatischen Ketonen gelingt dabei in guten Ausbeuten von 63-87\% und Diastereoselektivitäten, welche sich durchgängig im Bereich von 90:10 bewegen. Mit zunehmender Verzweigung in $\alpha$-Position sinkt die zu erwartende Ausbeute der Allylierung von fast $90 \%$ für das $\alpha$-unverzweigte Butanon (30a), über 59\% für das einfach $\alpha$-verzweigte 3-Methyl-2-butanon (30d) bis auf $22 \%$ 
für das doppelt $\alpha$-verzweigte Pinakolon (30e). Die Diastereoselektivität der Reaktion steigt dabei für 30d zunächst aufgrund der größeren sterischen Unterschiede zwischen dem Methyl- und dem Isopropylrest auf 96:4 an, fällt dann aber für 30e wieder auf 91:9 ab.

Zur Freisetzung der chiralen Homoallylalkohole $\mathbf{3 2}$ aus ihren Homoallylethern 54 muss eine reduktive Etherspaltung der benzylartigen Auxiliargruppe entweder unter den klassischen Birch-Bedingungen ${ }^{[29}{ }^{]}$oder unter ammoniakfreien Bedingungen mit 4,4'-Di-tert-butylbiphenyl (DBBP) ${ }^{[30]}$ durchgeführt werden (s. Schema 4-2).

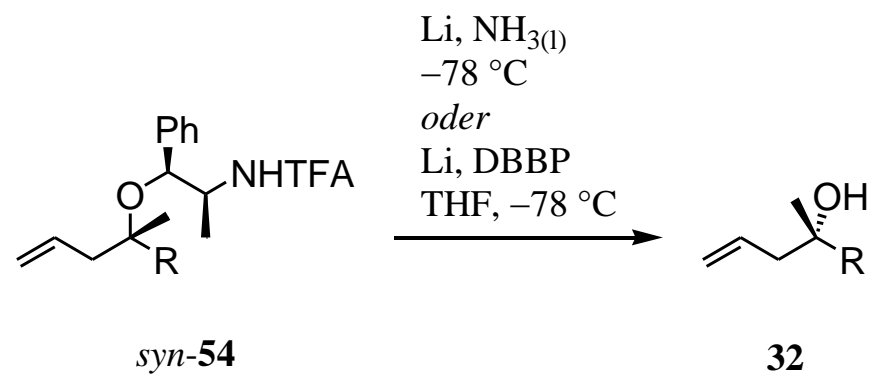

Schema 4-2: Reduktive Abspaltung der NPED-Auxiliargruppe unter Birch-Bedingungen.

Damit benötigt die MCAR nach Tietze im Vergleich zu anderen Allylierungsmethoden eine zusätzliche synthetische Stufe zur Abspaltung der Auxiliargruppe. Zur Steigerung der Effizienz der Reaktion hinsichtlich einer synthetischen Anwendbarkeit sollte die Auxiliargruppe selbst als Schutzgruppe dienen. Auf diese Weise würde die in einer Synthese meistens erforderliche Schützung des tertiären Alkohols entfallen, und damit sogar einen zusätzlichen Vorteil der MCAR nach Tietze einbringen. Jedoch die begrenzte Hydrolysestabilität der Trifluoracetamid-Funktionalität der NPED-Auxiliargruppe schränkt dessen Verwendbarkeit als Schutzgruppe deutlich ein.

Es wurden daher neue Auxiliare entwickelt, welche zum einen beispielsweise durch eine enantioselektive Reduktion in beiden enantiomeren Formen leicht zugänglich sind und zum anderen eine verbesserte Schutzgruppenstabilität ausweisen sollte (s. Kapitel 4.2.ff). 


\subsubsection{Totalsynthese des polyoxygenierten Cembrens 59}

Ein Anwendungsbeispiel für die MCAR nach Tietze mit dem (+)-Mandelsäure abgeleiteten (S)-Auxiliar 56, welches bei ähnlichen Selektivitäten wie das bereits vorgestellte NPED-Auxiliar $\mathbf{5 3}$ deutlich verbesserte Ausbeuten zeigt, ${ }^{[31]}$ ist die Totalsynthese des polyoxygenierten Cembrens 59. ${ }^{[32]}$ Zur Steigerung der Selektivität der Allylierungsreaktion des Ketons 55 wurde diese anstatt bei den üblichen $-78{ }^{\circ} \mathrm{C}$ bei bis zu $-196{ }^{\circ} \mathrm{C}$ durchgeführt. Auf diese Weise konnte der Homoallylether 57 in einem hervorragenden Epimerenverhältnis von 95:5 dargestellt werden. Aufgrund der bereits erwähnten mangelnden Schutzgruppeneigenschaften der Auxiliargruppe wurde diese direkt im Anschluss unter Verwendung der amoniakfreien Birch-Bedingungen entfernt. Somit konnte der entsprechende Homoallylalkohol 58 über zwei Stufen in einer Ausbeute von 71\% und 90\% ee erhalten werden. Weitere 18 Schritte inklusive einer Ringschlussmethatese waren nötigt, um das polyoxygenierte Cembrem $\mathbf{5 9}$ zu erhalten.

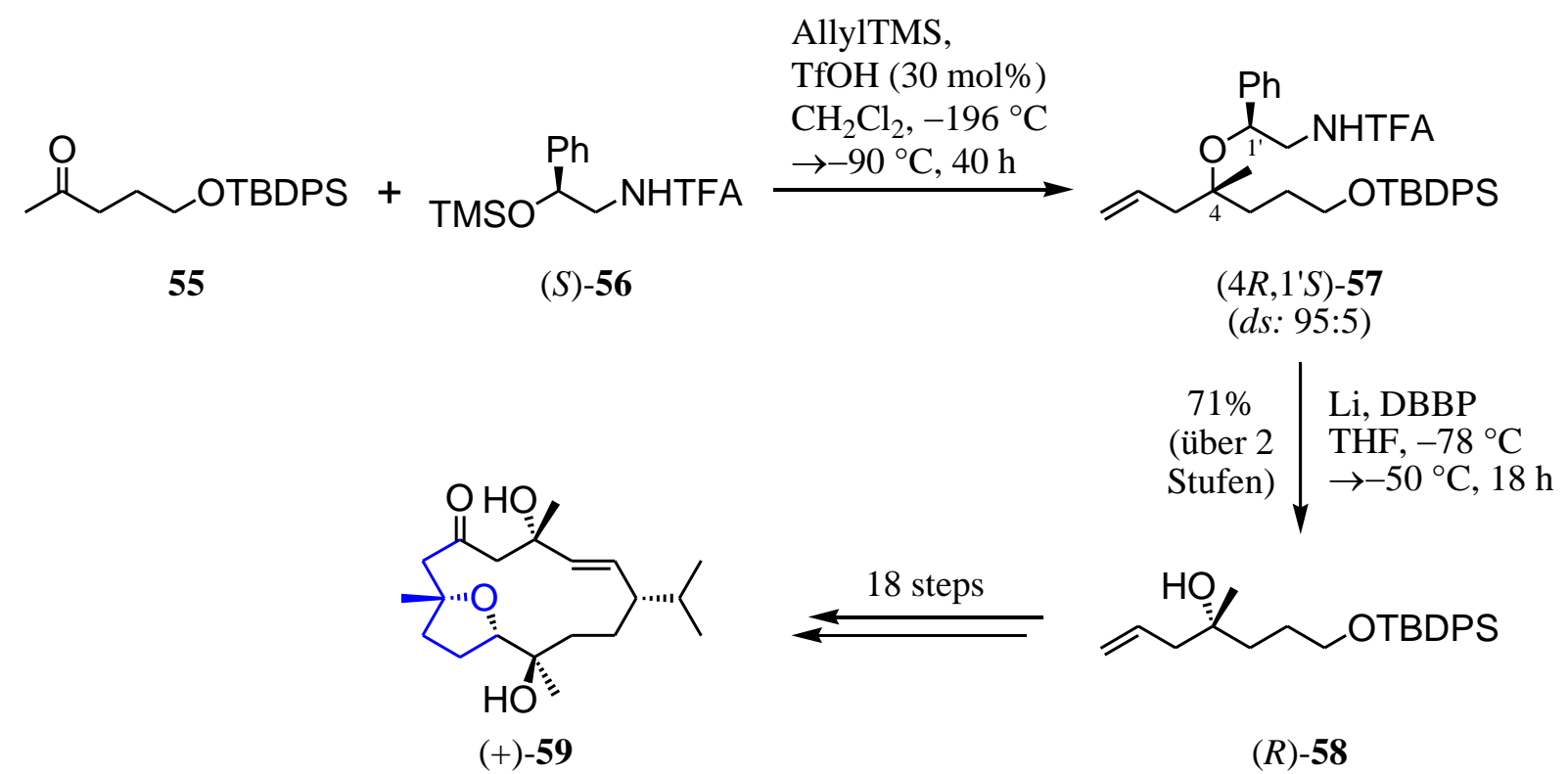

Schema 4-3: Ausschnitt aus der Totalsynthese des polyoxygenierten Cembrens 59 nach Tietze. ${ }^{[32]}$ 


\subsection{MCAR von Ketonen nach Tietze mit dem Phenyl-Benzyl-Auxiliar}

Anhand der von Tietze et al. durchgeführten Untersuchungen zur Topizität der Auxiliarstruktur, konnte das das Phenyl-Benzyl-Auxiliar (60) entwickelt werden. ${ }^{[33]}$ Dies vereint sowohl die hohe Diastereoselektivität der Reaktion mit dem NPED-Auxiliar (53) mit den erforderlichen Stabilitätsanforderungen, um als Schutzgruppen eingesetzt werden zu können. Die Resultate der Allylierungen der prochiralen Ketone 30 mit dem Phenyl-Benzyl-Auxiliar wurden in Tabelle 4-2 zusammengefasst.

Tabelle 4-2: Ergebnisse der Allylierung nach Tietze mit dem Phenyl-Benzyl-Auxiliar 60.

$\underset{\mathrm{R}}{\mathrm{O}}+\mathrm{NTMS}_{\mathrm{TMSO}} \stackrel{\mathrm{Ph}}{\mathrm{Ph}} \stackrel{\mathrm{TfOH}(20 \mathrm{~mol} \%)}{\mathrm{CH}_{2} \mathrm{Cl}_{2},-78{ }^{\circ} \mathrm{C}}$ 30

47

$(R)-60$

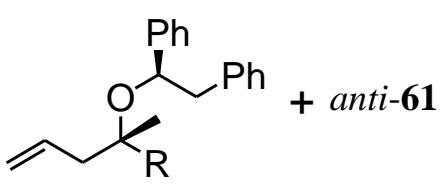

syn-61

\begin{tabular}{c|c|l|c|c|c}
\hline Nr. & Keton & $\mathbf{R}$ & Homoallylether & Ausbeute [\%] & syn:anti \\
\hline 1 & 30a & Et & 61a & 93 & $90: 10$ \\
\hline 2 & 30b & Pent & 61b & 93 & $88: 12$ \\
\hline 3 & 30c & $\left(\mathrm{CH}_{2}\right)_{2} \mathrm{Ph}$ & $\mathbf{6 1 c}$ & 76 & $86: 14$ \\
\hline 4 & 30d & $i-\mathrm{Pr}$ & $\mathbf{6 1 d}$ & 91 & $96: 4$ \\
\hline 5 & 30e & $t-\mathrm{Bu}$ & $\mathbf{6 1 e}$ & 8 & $72: 28$ \\
\hline 6 & 30f & $\mathrm{Ph}$ & $\mathbf{6 1 f}$ & 6 & $93: 7$ \\
\hline
\end{tabular}

Die Allylierung von $\alpha$-unverzweigten und einfach $\alpha$-verzweigten Methylketonen 30a-d mit dem Phenyl-Benzyl-Auxiliar 60 lieferte im direkten Vergleich zum NPED-Auxiliar nahezu identische Diastereoselektivitäten bei deutlich verbesserten Ausbeuten. Doppelt $\alpha$-verzweigte Ketone, wie Pinakolon (30e), $\alpha, \beta$-ungesättigte und aromatische Ketone, wie Acetophenon (30f), sind wie bereits bei Verwendung des NPED-Auxiliars (53) gefunden, keine geeigneten Substrate der diastereoselektiven MCAR-Reaktion nach Tietze.

Neben der üblichen reduktiven Abspaltung der Auxiliargruppe unter BirchBedingungen steht im Fall der Allylierung mit dem Phenyl-Benzyl-Auxiliar die Entschützungsmethode per Hydrogenolyse zur Verfügung. Aufgrund dessen, dass 
Doppelbindungen unter den hydrogenolytischen Bedingungen ebenfalls hydriert werden, kommt diese Methode - wenn dessen Hydrierung nicht erwünscht ist - erst nach deren Funktionalisierung zum Einsatz.

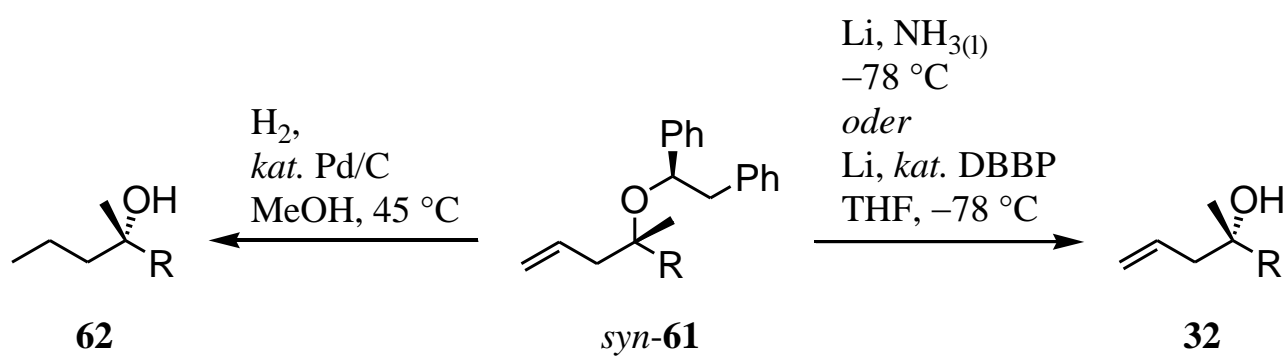

Schema 4-4: Reduktive Abspaltung der Phenyl-Benzyl-Auxiliargruppe durch Hydrogenolyse oder unter Birch-Bindungen.

\subsubsection{Totalsynthese von (-)-Taurospongin A}

In der Totalsynthese von Taurospongin A (67) verwendeten Robertson et al. ${ }^{[34 a]}$ für den stereoselektiven Aufbau des chiralen tertiären Alkoholmotivs die MCAR nach Tietze mit dem Phenyl-Benzyl-Auxiliar (60). Unter den üblichen Reaktionsbedingungen konnte das chirale Keton $\mathbf{6 3}$ mit einer Ausbeute von $68 \%$ bzw. unter Berücksichtigung der nach Beendigung der Reaktion zurückgewonnenen Menge an 63 sogar von $92 \%$ zum Homoallylether syn-64 umgesetzt werden.

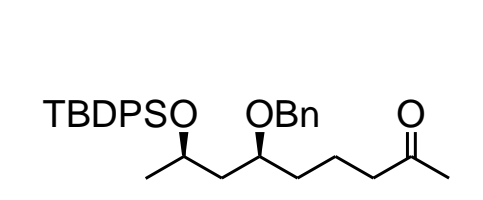

63
(R)-60, AllylTMS, $\mathrm{TfOH}(20 \mathrm{~mol} \%)$

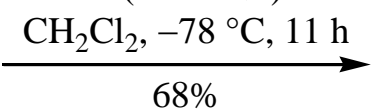

(92\% brsm)

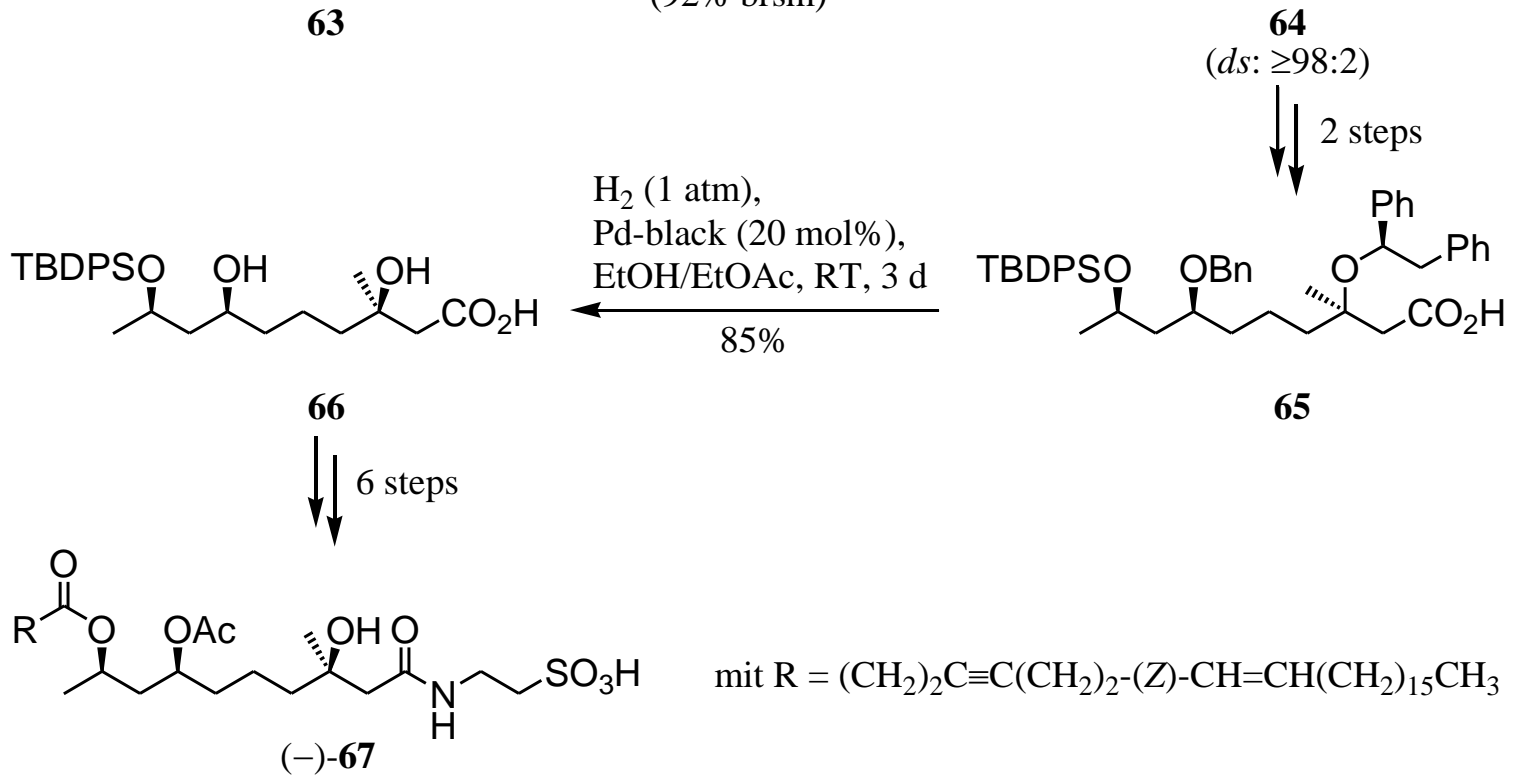

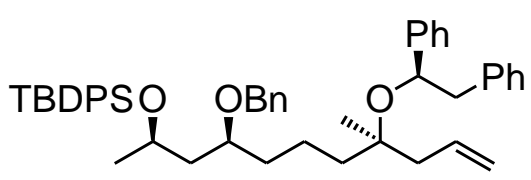

64

$\geq 98: 2)$

$\mathrm{H}_{2}(1 \mathrm{~atm})$ $85 \%$ 
Da die Bildung des Minderdiastereomers anti-64 nicht nachweisbar war $\left({ }^{13} \mathrm{C} \mathrm{NMR}\right)$, konnte man davon ausgehen, dass die Diastereoselktivität der Reaktion bei $\geq 98: 2$ lag. Zur Entfernung der Auxiliargruppe verwendeten Robertson et al. die bereits beschriebene Möglichkeit der Hydrogenolyse und führten diese simultan mit der Entschützung einer anderen Benzylgruppe durch. Nach der diastereoselektiven Allylierung von 63 konnte Taurospongin A (67) aus 64 in neun weiteren Schritten erhalten werden.

\subsubsection{Mechanismus der MCAR nach Tietze}

Der mechanistische Verlauf von Multikomponenten-Allylierungsreaktionen soll am Beispiel der MCAR nach Tietze mit dem Phenyl-Benzyl-Auxiliar (60) verdeutlicht werden. Im ersten Schritt wird säurekatalysiert aus der eingesetzten Carbonylverbindung, in diesem Fall dem Methylketon 30, und dem chiralen Silylether, hier 60, unter [1,3]-Silylwanderung das gemischte Acetal $\mathbf{6 8}$ gebildet. ${ }^{[35]}$ Durch anschließende säurekatalysierte Eliminierung von TMSOH entstehen intermediär die Oxeniumionen E-69 und Z-69 (partieller C-O-Doppelbindungscharakter). Da die Methylgruppe den geringeren sterischen Anspruch hat, wird bevorzugt das E-Isomer gebildet. Die relative Konformation der Auxiliargruppe zum „Keton“ ergibt sich dabei aus den geringsten sterischen Wechselwirkungen zwischen dem sich am Stereozentrum befindliche Wasserstoffatom und der Methylgruppe (für E-69) bzw. dem Rest (Z-69). Das irreversible Abfangen dieser reaktiven Intermediate mit Allyltrimethylsilan (47) unter Bildung der $\beta$-Silylcarbokationen ${ }^{[36]}$ syn-70 und anti-70 ist der eigentliche stereoinduzierende Schritt der Reaktion. Dadurch dass die Phenylgruppe den einen Halbraum stärker abschirmt als die Benzylgruppe, erfolgt der Angriff für E-69 bevorzugt über die Si-Seite unter Bildung von syn-70 und für Z-69 bevorzugt über die $R e$-Seite unter Bildung von anti-70. Durch einen nucleophilen Angriff von beispielsweise TMSOH am Siliciumatom von syn-70 bzw. anti-70 werden die Homoallylether syn-61 bzw. anti-61 und als Nebenprodukt $\mathrm{TMS}_{2} \mathrm{O}$ gebildet (s. Schema 4-6). 


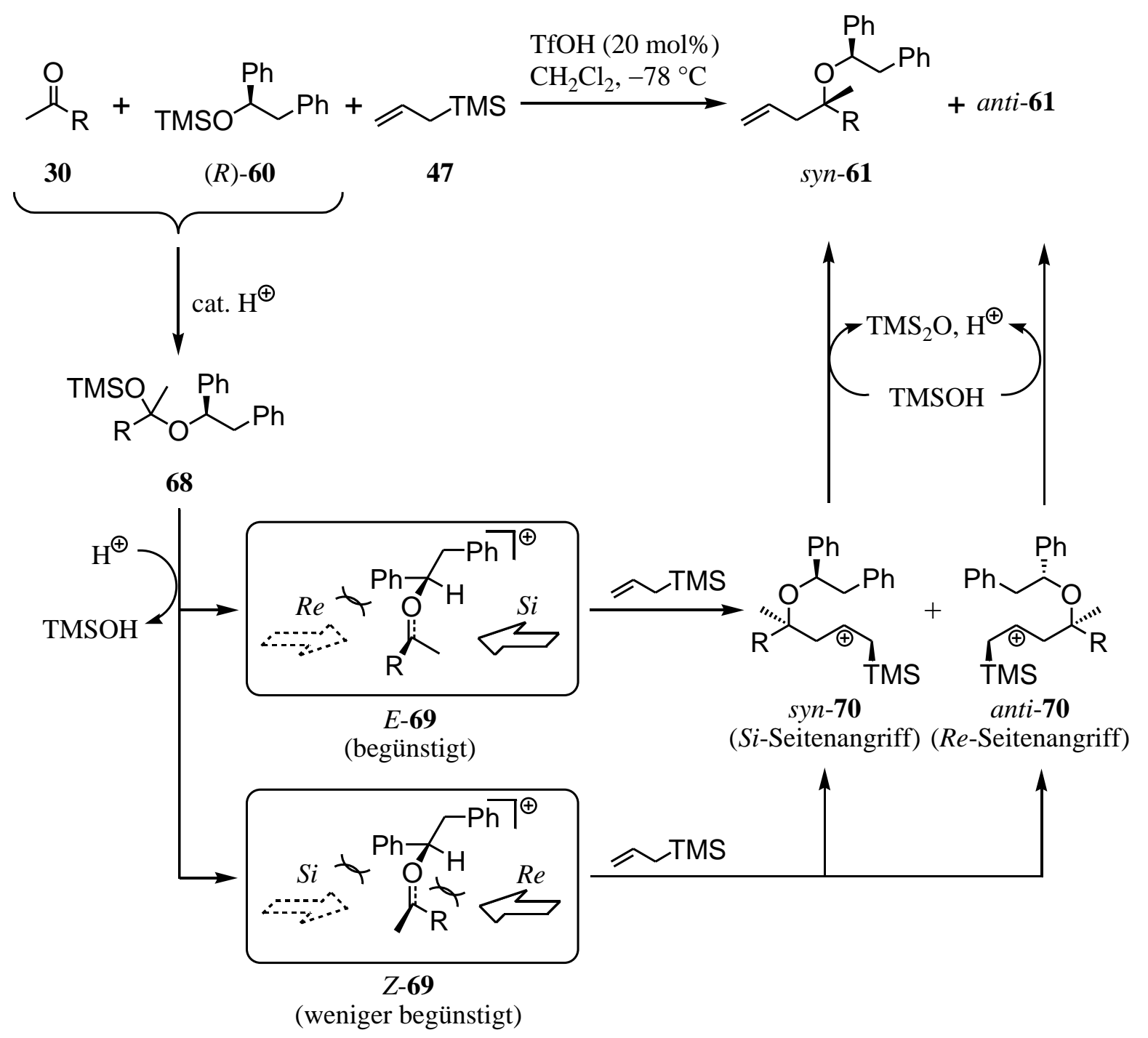

Schema 4-6: $\quad$ Mechanismus der MCAR nach Tietze mit dem Phenyl-Benzyl-Auxiliar (60). ${ }^{[33]}$

Zur Abschätzung der Diastereoselektivität einer Reaktion benötigt man die Aktivierungsenergien $\Delta G^{\ddagger}$ der Übergangszustände des stereoinduzierenden Schrittes. Anhand derer lassen sich relativen Geschwindigkeitskoeffizienten $k_{r e l}$ ableiten. Aufgrund der geringsten Aktivierungsenergie $\Delta G_{0}^{\ddagger}$ ergibt sich für den energetisch günstigsten Übergangszustand $k_{r e l}=1$. Folglich gilt für alle anderen $0<k_{r e l}<1$. Die Diastereoselektivität $d s$ der Reaktion ergibt sich schließlich aus dem Verhältnis der Summen der relativen Geschwindigkeitskonstanten, die zur Bildung des jeweiligen Diastereomers (syn, anti) beitragen (s. Gl. 1).

$$
k_{\text {rel }}=\exp \left(-\frac{\Delta G^{\ddagger}-\Delta G_{0}^{\ddagger}}{R T}\right) ; \quad d s=\sum_{\text {syn }} k_{\text {rel }} / \sum_{\text {anti }} k_{\text {rel }}
$$


Als relevant gelten dabei alle Übergangszustände, welche nicht mehr als $10 \mathrm{~kJ} \mathrm{~mol}^{-1}$ vom enenrgetisch günstigsten abweichen. Anhand von computergestützten Modellrechnungen lassen sich die Aktivierungsenergien mit Methoden wie Dichtefunktionaltheorie (DFT) von zuvor geometrioptimierten Grund- und Übergangszuständen abschätzen. Für den Fall der MCAR nach Tietze mit dem Phenyl-Benzyl-Auxiliar (60) sind solche Rechnungen wegen der Komplexität mit einer Vielzahl von möglichen relevanten Übergangszuständen sehr aufwändig. Abb. 4-2 zeigt, dass bereits für Butanon bis zu 96 verschiedene Übergangszustände betrachtet werden müssen.

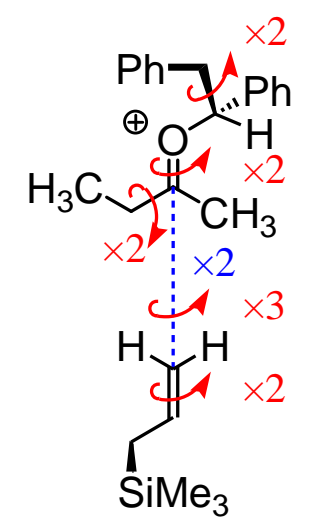

$$
\begin{array}{ll}
2 & \begin{array}{l}
\text { diastereomere Oxeniumionen }(E-\& Z \text {-Isomer }) \\
2
\end{array} \\
2 & \begin{array}{l}
\text { mögliche Konformationen der Auxiliargruppe }(+s c, a p) \\
\text { mögliche Konformationen des Ethylrestes }( \pm a c)
\end{array} \\
\hline 8 & \text { mögliche Ausgangskonformationen für das Oxeniumions } \\
2 & \text { prochirale Seiten des Oxeniumions }(\mathrm{Re}, \mathrm{Si}) \\
2 & \text { prochirale Seiten des Allylsilans }(\mathrm{Re}, \mathrm{Si}) \\
3 & \text { mögliche Angriffskonformationen }( \pm s c, a p) \\
\hline 12 & \text { verschiedene Möglichkeiten für den Angriff des Silans }
\end{array}
$$

Abb. 4-2: Ableitung der möglichen 96 stereoinduzierenden Übergangszustände bei der Allylierung von Butanon (30a) mit der MCAR nach Tietze. 


\section{$5 \quad$ Zielsetzung und Planung der Arbeit}

Die asymmetrische Multikomponenten-Allylierungsreaktion (MCAR) nach Tietze ist eine hervorragende Methode zur stereoselektiven Darstellung von tertiären Homoallylalkoholen. Der stereogene Schritt der Reaktion, die Addition von Allyltrimethylsilan (47) an den Carbonylkohlenstoff eines prochirales, aliphatischen Ketons, wie Butanon (30a) erfolgt dabei unter Stereokontrolle eines kovalentgebundenen chiralen Auxiliars, wie( $R$ )-60. Die auf diese Weise hochselektiv aufgebauten Homoallylether 61a können als geschützte Homoallylalkohole angesehen werden, wobei die zuvor zur Stereokontrolle verwendete Auxiliargruppe im Anschluss als Schutzgruppe weitere Verwendung findet.

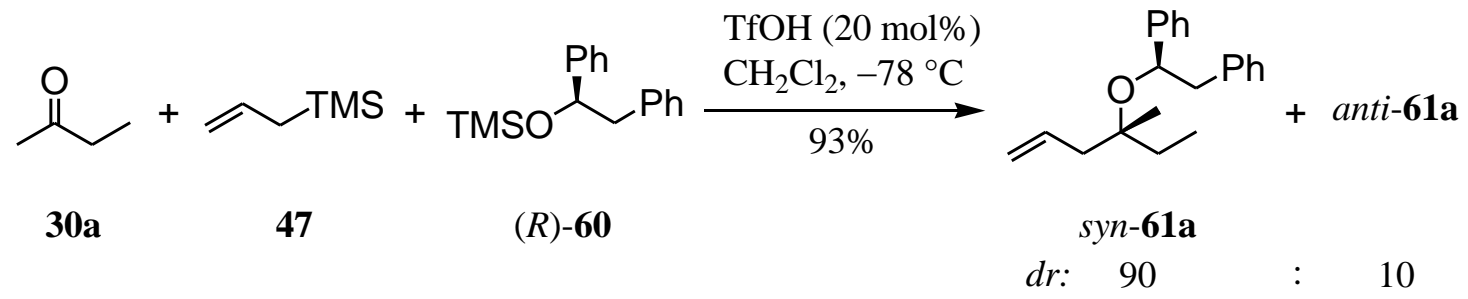

Schema 5-1: MCAR nach Tietze von Butanon (30a) mit dem Phenyl-Benzyl-Auxiliar (60).

Die zuverlässige Vorhersage der absoluten Konfiguration aufgrund des bekannten Mechanismus der Stereoinduktion und die Weiterentwicklung der Auxiliargruppe hinsichtlich ihrer Schutzgruppenqualität macht die MCAR nach Tietze mit dem Phenyl-Benzyl-Auxiliar (60) zu einer effizienten Methode für die Totalsynthese von Naturstoffen. Im Rahmen dieser Dissertation sollte die Verwendbarkeit des Verfahrens in der Totalsynthese der niedermolekularen Naturstoffe Furopinnatin (24) und Pinnatolid (25) erprobt werden.

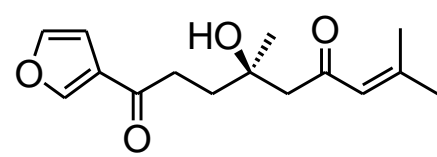

$(R)-24$

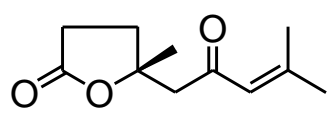

$(R)-25$<smiles>[R7]C(=O)CC[C@@](C)(CC)CC(=O)C=C(C)C</smiles>

$\mathrm{R}^{1}=$ Furyl, $\mathrm{R}^{2}=\mathrm{H}:(R)-\mathbf{2 4}$

$\left(\mathrm{R}^{1}, \mathrm{R}^{2}\right)-\gamma$-Lacton: $(R)-\mathbf{2 5}$

Abb. 5-1: (R)-Furopinnatin (24),(R)-Pinnatolid (25) und ihr gemeinsames Grundgerüst. 
Beide Verbindungen wurde von Bohlmann et al. u.a. aus Athanasia Pinnata isoliert ${ }^{[5]}$ und wurden bisher nicht in enantioselektiver Form synthetisiert. So sind weder die absolute Konfiguration der Naturstoffe noch ihre chiroptischen Eigenschaften wie Zirkulardichroismus (CD), optische Rotationsdispersion (ORD) oder der Drehwert bekannt.

\subsection{Retrosynthetische Analyse}

Aufgrund der strukturellen Verwandtschaft von Pinnatolid (25) und Furopinnatin (24) sollte der synthetische Ansatz so gewählt werden, dass beide Verbindungen auf eine gemeinsame Vorstufe zurückgeführt werden können. Dazu wurde zunächst das 3-Acylfuranmotiv von Furopinnatin (24) auf das mit Pinnatolid (25) gemeinsame Strukturelement des $\gamma$-Lactons reduziert, da sich dieses durch Addition einer 3-Furylmetall-Verbindung $\mathrm{zu}$ dem gewünschten Acylfuran öffnen lassen sollte. ${ }^{[50]}$ Diese Additionsreaktion ist aufgrund der geringere Reaktivität der Carboxylgruppe des $\gamma$-Lactons nur in Abwesendheit der reaktiveren Carbonylgruppe der EnonFunktionalität möglich. Da aus ökonomischer (zusätzliche Stufen) und ökologischer Sicht (zusätzlicher Abfall) weitgehend auf den Einsatz von Schutzgruppen verzichtet werden sollte, wurde der Allylalkohol 71 als gemeinsame Vorstufe gewählt, welcher durch Oxidation in die Enonfunktionalität von Pinnatolid (25) und Furopinnatin (24) überführbar sein sollte (s. Schema 5-2).

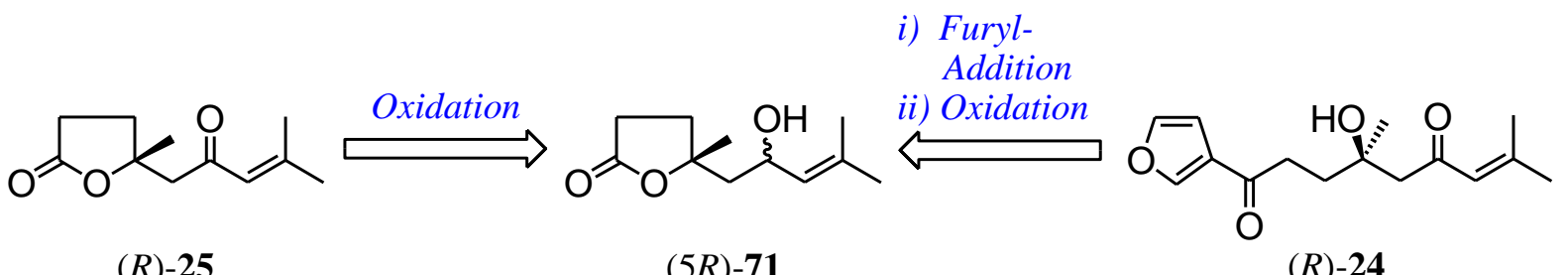

$(R)-25$

$(5 R)-71$

$(R)-24$

Schema 5-2: Retrosynthetische Rückführung von Pinnatolid (25) und Furopinnatin (24) auf die gemeinsame Vorstufe 71.

Zum enantioselektiven Aufbau des tertiären Alkoholmotivs von 71 sollte die stereoselektive Allylierung nach L. F. Tietze eines geeigneten prochiralen Methylketons mit dem enantiomerenreinen Phenyl-Benzyl-Auxiliar (60) eingesetzt werden. Dabei sollte eine der beiden Kohlenstoffketten des tertiären Alkoholmotivs durch den Alkylrest des Methylketons eingeführt werden, während die andere durch nachfolgende synthetische Modifikationen der Allylgruppe erhalten werden sollte. 
Damit ergeben sich für die weitere Retrosynthese der gemeinsamen Vorstufe71 zwei Möglichkeiten:

- Retrosyntheseweg A: Asymmetrische Allylierung von Sulcaton(76) und Aufbau des $\gamma$-Lactonrings aus der Allylgruppe oder

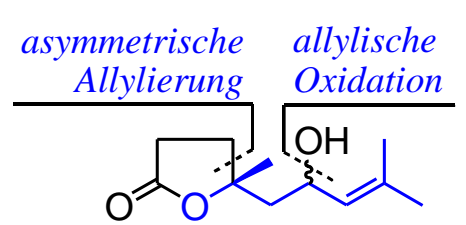

(5R)-71
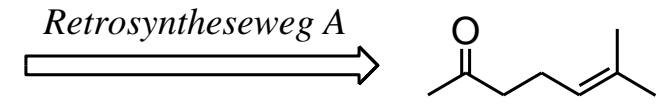

76

Schema 5-3: $\quad$ Retrosynthetische Ableitung von Sulcaton (76) aus der Vorstufe (5R)-71.

- Retrosyntheseweg B: Asymmetrische Allylierung eines Lävulinsäureesters 86 und Aufbau der Allylalkoholseitenkette aus der Allylgruppe.

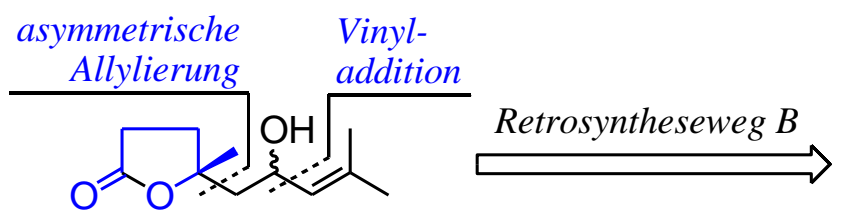

(5R)-71

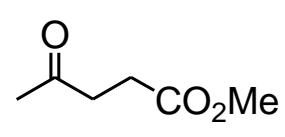

$86 a$

Schema 5-4: $\quad$ Retrosynthetische Ableitung des $\beta$-Ketoester 86a aus der Vorstufe (5R)-71.

\subsubsection{Retrosyntheseweg A}

Der Retrosyntheseweg A sieht zur Einführung der Hydroxylgruppe der Vorstufe71 eine regioselektive allylische Oxidation des $\gamma$-Lactons 82 vor. Durch Öffnung des Lactonrings, Überführung der so gebildeten Carbonsäure-Seitenkette in eine Allylgruppe und nachfolgende retrosynthetische Entfernung der Allylgruppe erhält man das prochirale Keton 6-Methyl-2-hept-5-enon (76, Trivialname: Sulcaton). Die Allylierung von 76 mit dem Allylsilan 47 in Gegenwart des Auxiliars $(R)-60$ sollte nach dem Tietze-Verfahren den Homoallylether $(R, R)-77$ geben, welcher durch eine regioselektive Hydroborierung und nachfolgenden Oxidationen in die Carbonsäure $(R, R)-\mathbf{8 0}$ überführbar sein sollte.

Da die absolute Konfiguration von Pinnatolid (25) und Furopinnatin (24) unbekannt und das für die Synthese des $(R)$-Phenyl-Benzyl-Auxiliars eingesetzte DiphenylL-prolinol im Gegensatz zu seiner D-Form in größeren Mengen vorhanden war, wurde 
in diesem Ansatz die unter der Verwendung des $(R)$-Auxiliars erzielbare $S$-Konfiguration für 25 und 24 als Zielstruktur angenommen (s. Schema 5-5).

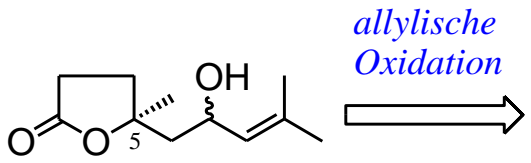

$(5 S)-71$

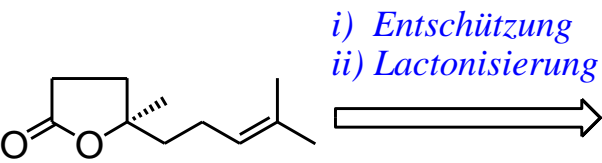

$(R)-82$

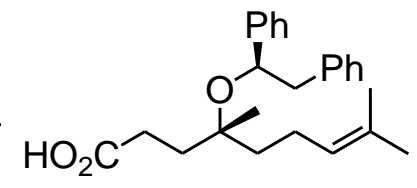

$(R, R)-80$

i) Hydroborierung

ii) Oxidation

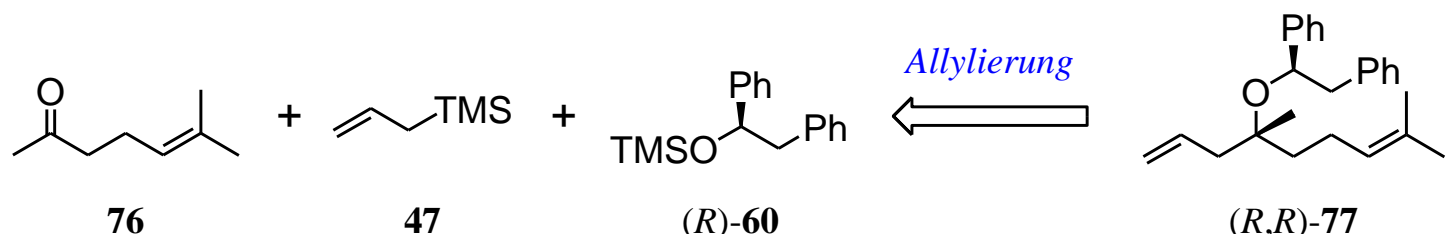

Schema 5-5: Retrosyntheseweg A.

\subsubsection{Retrosyntheseweg B}

Der Retrosyntheseweg B beinhaltet die Rückführung der Allylalkohol-Seitenkette von 71 über eine Aldehydgruppe auf eine Allylgruppe. Das Strukturmotiv des $\gamma$-Lactons sollte ebenfalls durch Lactonisierung einer Carboxylfunktion - beispielsweise eines Methylesters - mit der tertiären Hydroxylgruppe gebildet werden. Nach retrosynthetischer Entfernung der Allylgruppe ergibt sich demzufolge als Substrat für die Allylierung der $\gamma$-Ketoester Lävulinsäuremethylester (86a).

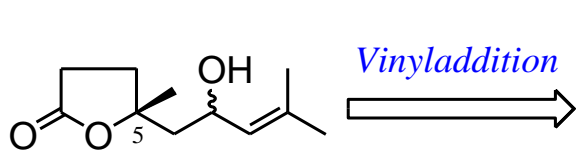

$(5 R)-71$

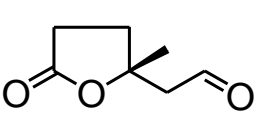

$(R)-99$

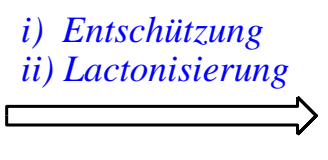

wa.<smiles>COC(=O)CCC(C)(CC=O)OC(Cc1ccccc1)c1ccccc1</smiles>

$(R, R)-95$

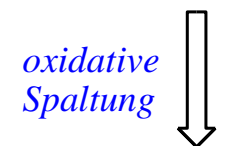<smiles>CC(=O)CCC(C)=O</smiles>

Allylierung<smiles>C=CCC(CCC(C)=O)(CC(c1ccccc1)c1ccccc1)OC(C)C</smiles>

$(R, R)-89 \mathbf{a}$

Schema 5-6: Retrosyntheseweg B. 
Die Umsetzung von 86a mit dem Allylsilan 47 in Gegenwart des Auxiliars $(R)$-60 sollte unter den Reaktionsbedingungen der MCAR nach Tietze den Homoallylether $(R, R)$-89a liefern, welcher nach oxidativer Spaltung der allylischen Doppelbindung und Abspaltung der Auxiliargruppe den Aldehyd $(R)-99$ ergeben sollte. Durch Addition eines vinylartigen Metallorganyls an dessen Aldehydfunktion sollte demzufolge die 5R-Form von $\mathbf{7 1}$ und damit auch von $\mathbf{2 4}$ und 25 zugänglich sein (s. Schema 5-6).

\subsubsection{Definition der Teilaufgaben}

Aus den vorangegangenen Überlegungen ergaben sich folgende Teilaufgaben:

- Synthese des $(R)$-Phenyl-Benzyl-Auxiliars (60) in enantiomerenreiner Form.

- Untersuchung zur Diastereoselektivität der MCAR nach Tietze mit dem PhenylBenzyl-Auxiliar (60) hinsichtlich der Allylierung der verwendeten Ketone.

- Untersuchung geeigneter Verfahren zur Abtrennung des Minderdiasteromers.

- Vervollständigung der enantioselektiven Synthese von Furopinnatin (24) und Pinnatolid (25).

- Charakterisierung der chiroptischen Eigenschaften von Furopinnatin (24) und Pinnatolid (25). 


\section{Darstellung der Ergebnisse}

\subsection{Synthese des $(R)$-Phenyl-Benzyl-Auxiliars}

Das enantiomerenreine $(R)$-Auxiliar $\mathbf{6 0}$ wurde ausgehend von käuflich erhältlichem Desoxybenzoin (72) synthetisiert. Dazu wurde das prochirale Keton $\mathbf{7 2}$ in einer abgewandelten Corey-Itsuno-Reduktion ${ }^{[37,38]}$ mit Boran-Dimethylsulfid-Komplex als Reduktionsmittel in Gegenwart des Organokatalysators Diphenyl-L-prolinol (73) ${ }^{[39]}$ in einem Toluol/THF-Gemisch bei $45^{\circ} \mathrm{C}$ über $1.5 \mathrm{~h}$ in quantitativer Ausbeute zu dem enantiomerenangereicherten $\quad(R)$-1,2-Diphenylethanol (74) $\quad(84 \%$ ee) $\quad$ umgesetzt. Obwohl mit dem $(S)$-Me-CBS-Reagenz bei dieser Reaktion Selektivitäten von bis zu 92\% ee und Ausbeute von 84\% erreicht werden können, ${ }^{[33 a]}$ wurde aus ökonomischen Gründen dennoch der kostengünstigeren Variante der Vorzug gegeben, da diese bilanziert auf die gebildete Menge an $(R)$-Enantiomer die höhere Ausbeute (84\% zu 77\%) lieferte. Im Anschluss wurde ohne weitere Aufreinigung von $(R)-\mathbf{7 4}$ mit 3,5-Dinitrobenzoesäurechlorid (DNBCl) in Gegenwart von katalytischen Mengen an 4-Dimethylaminopyridin (DMAP) und Triethylamin als Base in Dichlormethan bei Umgebungstemperatur in 95\% Ausbeute verestert. Nach Umkristallisation aus Essigester $/ n$-Heptan konnte der Dinitrobenzoesäureester $(R)-\mathbf{7 5}$ in $72 \%$ als reines Enantiomer (>99\% ee) erhalten werden.

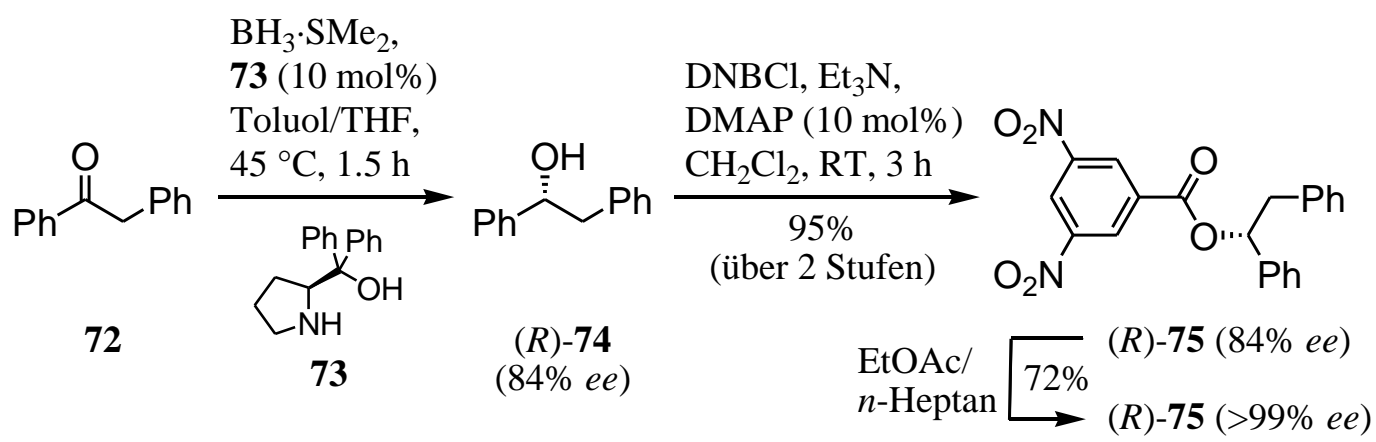

Schema 6-1: Enantioselektive Reduktion von 72 mit anschließender Veresterung von $(R)-\mathbf{7 4}$ zum Dinitrobenzoesäureester $(R)-\mathbf{7 5}$ und dessen Umkristallisation.

Bei der Trennung des angereicherten Enantiomers von der racemischen Verbindung durch Umkristallisation nutzt man aus, dass das Racemat in einer anderen Raumgruppe auskristallisiert als das reine Enantiomer. Während der Umkristallisation des Gemisches ist das Bestreben zur Bildung der einen oder anderen Kristallstruktur verschieden groß. Wie groß diese Unterschiede sind, lässt sich anhand der Differenz 
ihrer Schmelzpunkte abschätzen. Im Fall des Alkohols 74 beträgt diese lediglich $5{ }^{\circ} \mathrm{C}$, weshalb eine Trennung praktisch schwierig zu realisieren ist. Im Fall des Dinitrobenzoesäureesters 75 schmilzt das Enantiomer aber bereits $82^{\circ} \mathrm{C}$ früher als das Racemat. Die Trennung von Racemat und Enantiomer ist nun aufgrund dieser hohen Differenz leicht durchführbar. Dieses Beispiel macht deutlich, wie stark sich die intermolekularen Kräfte innerhalb der Kristallstrukturen von Racemat und Enantiomer unterscheiden können.

Zur Rückgewinnung des Alkohols $\mathbf{7 4}$ wurde der enantiomerenreine Dinitrobenzoesäureester $(R)$-75 mit Lithiumhydroxid-monohydrat in einem Lösungsmittelgemisch aus Dichlormethan, Methanol und einigen Tropfen Wasser bei Umgebungstemperatur in $1 \mathrm{~h}$ quantitativ hydrolysiert. Der so erhaltene enantiomerenreine Alkohol (R)-74 wurde mit Trimethylsilyltrifluormethansulfonat in Gegenwart von Triethylamin als Base in Dichlormethan bei Umgebungstemperatur in $2 \mathrm{~h}$ in $93 \%$ Ausbeute zum $(R)$-Auxiliar 60 umgesetzt.

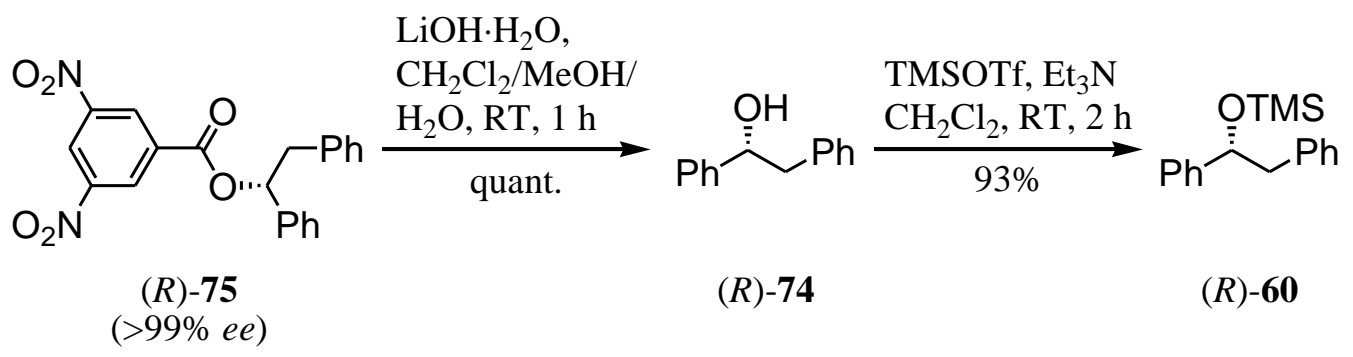

Schema 6-2: $\quad$ Hydrolyse des enantiomerenreinen Dinitrobenzoesäureesters $(R)-75$ und Bildung des $(R)$-Phenyl-Benzyl-Auxiliars (60) durch Silylierung des Alkohols $(R)-74$.

Die Bestimmung der Enantiomerenüberschüsse von $(R)$-74 wurde durch Vergleich mit der analytischen Trennung von rac-74 an der chiralen HPLC-Phase Chiralpak ${ }^{\circledR}$ IA mit dem isokratischen Eluenten $n$-Hexan / MTBE / $i$-PrOH 75:24.5:0.5 durchgeführt.
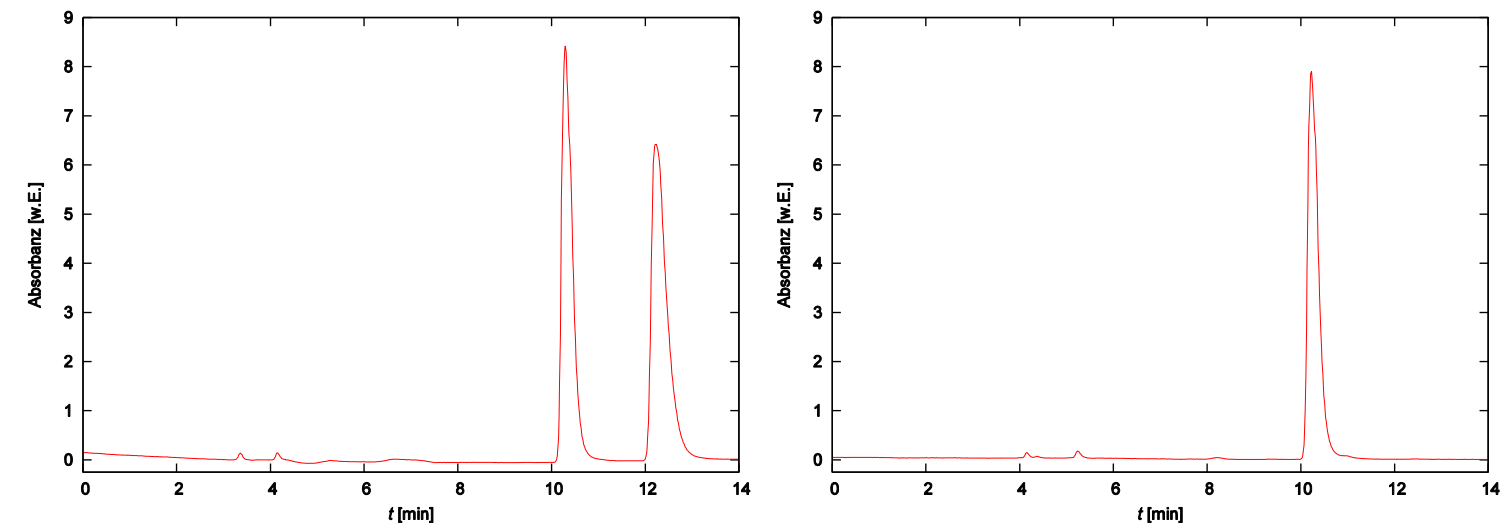

Abb. 6-1: $\quad$ HPLC-Chromatogramme von rac-74 (links) und (R)-74 (rechts). 


\subsection{Syntheseroute A: Allylierung von Sulcaton}

Analog zum Retrosyntheseweg A wurde das kommerziell erhältliche Sulcaton (76) als Ausgangsverbindung für die Synthese von Pinnatolid (25) und Furopinnatin (24) eingesetzt. Die Allylierung des prochiralen Ketons 76 mit Allyltrimethylsilan (47) in Dichlormethan bei $-78{ }^{\circ} \mathrm{C}$ in Gegenwart des $(R)$-Phenyl-Benzyl-Auxiliars (60) und katalytischen Mengen Trifluormethansulfonsäure lieferte den Homoallylether $(R, R)-\mathbf{7 7}$ in sehr guter Ausbeute von 95\% und einer guten Diastereoselektivität von ca. 90:10 $\left({ }^{13} \mathrm{C}-\mathrm{NMR}\right)$ (s. Schema 6-3).

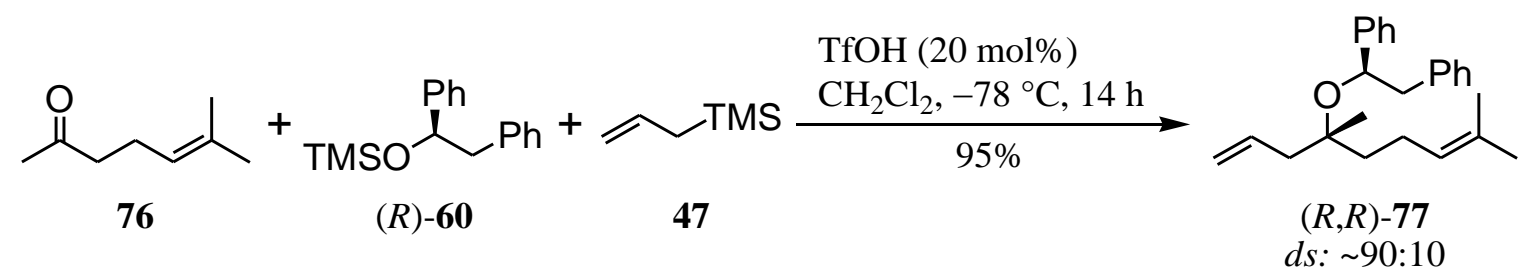

Schema 6-3: $\quad$ MCAR nach Tietze mit Methylketons 76 und dem Phenyl-Benzyl-Auxiliar $(R)-60$.

Allerdings war eine Trennung der beiden Epimere durch Umkristallisation nicht möglich. Eine chromatographische Trennung konnte weder an üblichem Kieselgel $(30-60 \mu \mathrm{m})$ noch an achiralen (Lichrosorb Si 60, Kromasil C-18) oder chiralen HPLC-Phasen (Ciralcel ${ }^{\circledR}$ OD, Chiralpak ${ }^{\circledR}$ IA, Chiralpak ${ }^{\circledR}$ IB) erzielt werden. Somit wurde entschieden, die Synthese zunächst mit dem Diastereomerengemisch fortzusetzen und gegebenenfalls auf einer späteren Stufe den Diastereomerenüberschuss weiter zu erhöhen.

Im Folgenden musste die terminale Doppelbindung der Allylfunktionalität regioselektiv hydratisiert werden. Eine solche ,anti-Markovnikow“-Addition von Wasser lässt sich mittels einer Hydroborierung (inkl. einer oxidativen Spaltung der C-B-Bindung) erreichen. Zur Steigerung der Regioselektivität bezüglich der terminalen gegenüber der höher substituierten internen Doppelbindung wurde die Hydroborierung mit dem sterisch anspruchsvolleren 9-Borabicyclo[3.3.1]nonan (9-BBN) anstelle von $\mathrm{BH}_{3}$ durchgeführt. Die Umsetzung des Homoallylethers $(R, R)-77$ mit 9-BBN in THF und anschließender oxidativer Aufarbeitung mit alkalischer $\mathrm{H}_{2} \mathrm{O}_{2}$-Lösung lieferte den Alkohol $(R, R)-78$ in einer guten Ausbeute von $86 \%$. Die Oxidation zur Carbonsäure $(R, R)-\mathbf{8 0}$ gelang über zwei Stufen in moderater Ausbeute von 60\%. Dazu wurde zunächst der Alkohol $(R, R)-78$ mit dem Dess-Martin- 
Periodinan $(\mathrm{DMP})^{[40]}$ in Dichlormethan zum entsprechenden Aldehyd oxidiert und dieser ohne weitere Aufarbeitung (Zersetzung an Kieselgel) durch eine PinnickOxidation $^{[41]}$ mit Natriumchlorit als Oxidationsmittel in DMF zur Carbonsäure $(R, R)-80$ oxidiert (s. Schema 6-4).

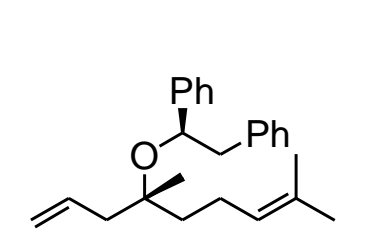

$(R, R)-77$

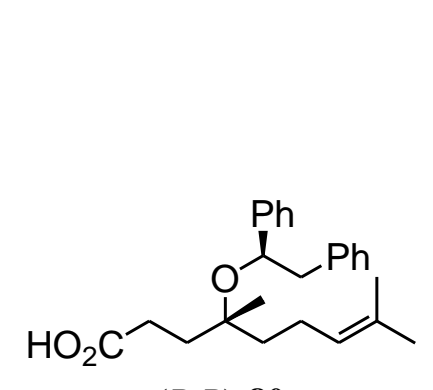

$(R, R)-80$ i) $9-\mathrm{BBN}$

THF, RT, $32 \mathrm{~h}$

ii) $\mathrm{H}_{2} \mathrm{O}_{2}, 3 \mathrm{~N} \mathrm{NaOH}$,

THF/ $\mathrm{H}_{2} \mathrm{O}, \mathrm{RT}, 2 \mathrm{~h}$ $86 \%$

$\mathrm{NaClO}_{2}$,

2-Methyl-2-buten,

$\mathrm{NaH}_{2} \mathrm{PO}_{4}(\mathrm{pH}=3.5)$

$\mathrm{DMF} / \mathrm{H}_{2} \mathrm{O}, \mathrm{RT}, 2 \mathrm{~h}$

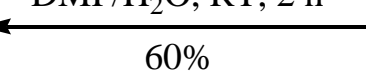

(über 2 Stufen)

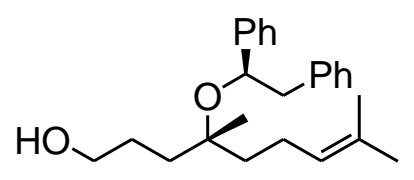

$(R, R)-78$
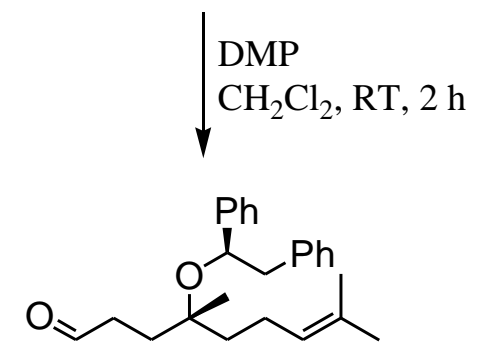

(R,R)-79

Schema 6-4: $\quad$ Regioselektive Hydroborierung von $(R, R)-77$ und zweistufige Oxidation des primären Alkohols $(R, R)-\mathbf{7 8}$ zur Carbonsäure $(R, R)-\mathbf{8 0}$.

Eine Trennung der beiden Epimere von $\mathbf{7 8}$ oder $\mathbf{8 0}$ war weder chromatographisch an Kieselgel, noch per Umkristallisation möglich. Auf eine zeitintensive Trennung per präparativer HPLC wurde zunächst verzichtet, da zunächst geprüft werden sollte, ob diese Syntheseroute zielführend war.

Zur Abspaltung der Auxiliargruppe wurde die Säure 80 mit elementarem Lithium in flüssigem Ammoniak bei $-78^{\circ} \mathrm{C}$ (klassische Birch-Bedingungen) ${ }^{[29]}$ behandelt. Die dabei gebildete $\gamma$-Hydroxycarbonsäure $(R)$-81 wurde ohne weitere Reinigung mit Methyliodid und wasserfreiem Kaliumcarbonat als Base in $79 \%$ Ausbeute zum $\gamma$-Lacton 82 verestert (s. Schema 6-5).<smiles>CC(C)=CCC[C@]1(CCC(=O)O)CCC(=O)O1</smiles>

$(R, R)-80$

$(R)-81$

$(R)-82$

Schema 6-5: $\quad$ Abspaltung der Auxiliargruppe von $(R, R)-80$ unter Birch-Bedingungen und Lactonisierung der $\gamma$-Hydroxycarbonsäure $(R)-81$. 
Das $\gamma$-Lacton $(R)$-82 ist das Enantiomer des Naturstoffs, namens (-)-4,8-Dimethylnon-7-en-olid ${ }^{[42]}$, der von Kitaura et al. aus der japanischen Apfelsorte Orin isoliert worden war. Anhand eines Vergleichs der Drehwerte konnte die aufgrund des Mechanismus der Allylierungsreaktion angenommene $R$-Konfiguration bestätigt werden. Zur chromatographischen Bestimmung des Enantiomerenüberschusses wurde die Verbindung 82 nach einer literaturbekannten Methode über zwei Stufen ebenfalls in racemischer Form hergestellt (s. Schema 6-6).

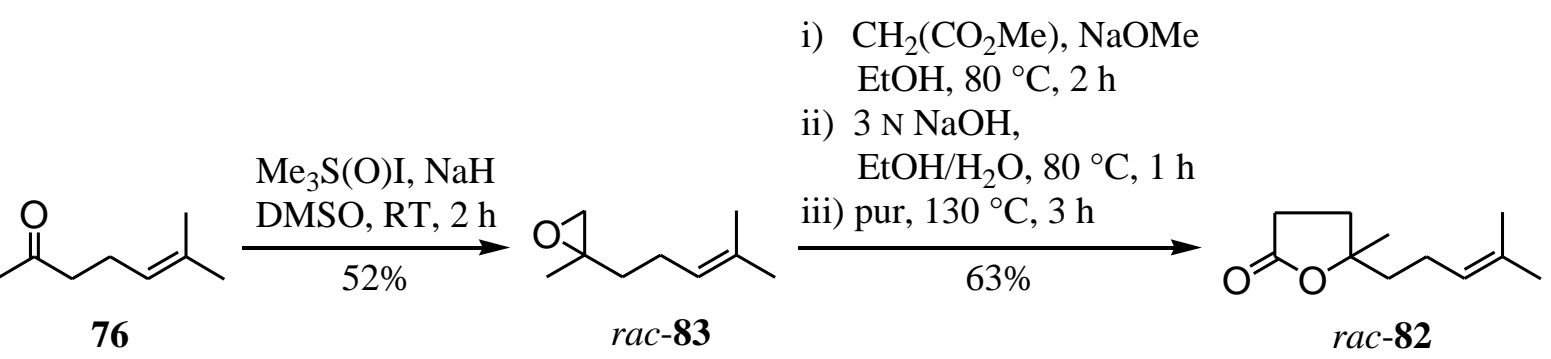

Schema 6-6: Zweistufige Synthese von rac-82 aus 6-Methyl-5-hepten-2-on (76). ${ }^{[42]}$

Als Ausgangsverbindung diente dabei ebenfalls das Keton 76, welches durch Reaktion mit einem in situ aus Trimethylsulfoxoniumiodid und Natriumhydrid hergestellten Schwefelylids in einer moderaten Ausbeute von 52\% in das Epoxid rac-83 überführt wurde. Epoxidöffnung mit Dimethylmalonat in ethanolischer Lösung in der Siedehitze, anschließende Verseifung mit Natronlauge und solvensfreie Decarboxylierung bei $130{ }^{\circ} \mathrm{C}$ lieferte das $\gamma$-Lacton rac-82 in einer guten Ausbeute von $63 \%$. DieTrennung der Enantiomeren von rac-82 konnte mittels GC an einer chiralen Cyclodextrin-Phase erreicht werden. Der Vergleich mit der Verbindung, welche über die Tietze-Allylierung stereoselektiv dargestellt wurde, ergab ein Enantiomerenverhältnis von 86:14 bzw. einen Enantiomerenüberschuss von 72\% ee.

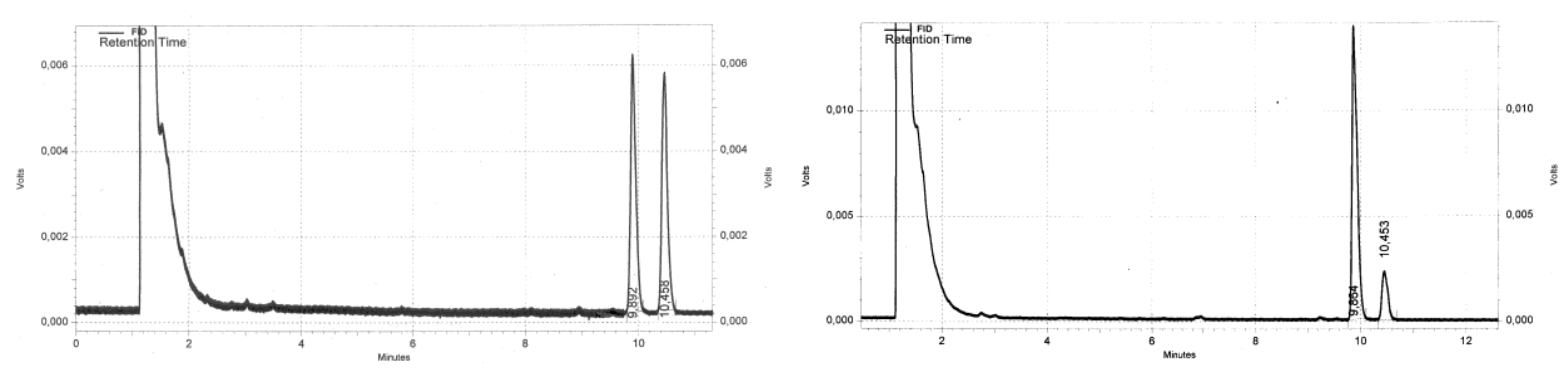

Abb. 6-2: GC-Chromatogramme von rac-82 (links) und (R)-82 (rechts). 
Die allylische Oxidation von 82 zu Pinnatolid (25) sollte regioselektiv an der internen allylischen Methylengruppe durchgeführt werden. Bei Verwendung der üblichen Selendioxid-basierten Verfahren ${ }^{[43 a]}$ würde die allylische Oxidation hingegen an den terminalen Methylgruppen erfolgen. Als mögliche geeignete Methoden wurden zwei radikal-basierte Verfahren mit tert-Butylhydroperoxid (TBHP) als Sauerstoffquelle untersucht. Die Radikale können dabei sowohl reduktiv mit Mangan(III)acetat ${ }^{[43 \mathrm{c}]}$ als auch oxidativ mit Pyridiniumdichromat $(\mathrm{PDC})^{[43 \mathrm{~b}]}$ aus TBHP erzeugt werden (s. Tabelle 6-1).

Tabelle 6-1: Synthesemethoden zur allylische Oxidation des $\gamma$-Lactons 82.

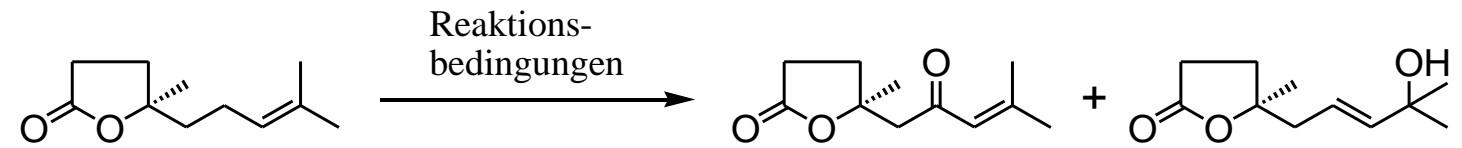

$(R)-82$

$(S)-25$

$(R)-84$

\begin{tabular}{|c|c|c|}
\hline Nr. & Reaktionsbedingungen & Ergebnisse \\
\hline 1 & $\begin{array}{l}\mathrm{Mn}(\mathrm{OAc})_{3} \cdot 2 \mathrm{H}_{2} \mathrm{O}(20 \mathrm{~mol} \%), \operatorname{TBHP}(5.0 \text { Äq. }) \\
\mathrm{MS} 4 \AA \text { A, EtOAc, RT, } 36 \mathrm{~h}\end{array}$ & 25: $31 \%, 84: 11 \%$ \\
\hline 2 & $\begin{array}{l}\text { PDC (2.0 Äq.), TBHP (2.0 Äq.) } \\
\text { Benzol, RT, } 4 \text { h }\end{array}$ & $\begin{array}{l}\text { 25: } 13 \%(30 \% \text { brsm }), \\
\text { 84: } \text { n.i. }\end{array}$ \\
\hline
\end{tabular}

Die Umsetzung von $\gamma$-Lacton 82 mit tert-Butylhydroperoxid und Mangan(III)-acetat in Essigester liefete das Enon Pinnatolid (25) in einer mäßigen Ausbeute von $31 \%$ und als Nebenprodukt den tertiären Allylalkohol 84 in 11\% Ausbeute. Die analoge Reaktion mit PDC in Benzol lieferte bei nicht vollständigem Umsatz lediglich eine isolierte Ausbeute von 13\%. Bilanziert auf die Reisolierung von 82 konnte das Enon 25 in einer vergleichbaren Ausbeute von $30 \%$ erhalten werden. Beide Oxidationsverfahren liefern neben 84, dessen Bildung sich anhand der Konjugation des intermediär gebildeten Allylradikals erklären lässt, ein Vielzahl von Zersetzungsprodukten. Bei Verwendung des enantiomerenangereichterten $\gamma$-Lactons $(R)$-82 war somit auch (S)-Pinnatolid (25) in $72 \%$ ee zugänglich.

Dennoch wurde diese Route für die Synthese des Furopinnatins nicht weiter verfolgt, da lediglich moderate Enantiomerenüberschüsse zu erwarten waren und die allylische Oxidation nur mit mäßigen Ausbeuten aufwarten konnte. 


\subsection{Syntheseroute B: Allylierung von Lävulinsäuremethylester}

Die zweite mögliche Ausgangsverbindung für die enantioselektive Synthese von Pinnatolid (25) und Furopinnatin (24) über die Allylierung nach Tietze war der Lävulinsäureester 86a. Die Allylierung von 86a mit Allyltrimethylsilan (47) in Dichlormethan bei $-78{ }^{\circ} \mathrm{C}$ in Gegenwart des $(R)$-Phenyl-Benzyl-Auxiliars (60) und katalytischen Mengen Trifluormethansulfonsäure gelang mit einer sehr guten Ausbeute von 91\%. Dabei wurde der Homoallylether $(R, R)$-89a in einem hervorragenden Diastereomerenverhältnis von ca. 94:6 ( $\left.{ }^{13} \mathrm{C}-\mathrm{NMR}\right)$ erhalten. Eine solche Diasteroselektivität konnte bisher nur für $\alpha$-verzweigte Methylketone, wie z.B. Methyl-iso-propylketon (30d) (s. Tabelle 4-2) erreicht werden.

\subsubsection{Untersuchung der Tietze-Allylierung von prochiralen Ketoestern}

Dieser Befund begründete die folgenden Untersuchungen zum Allylierungsverhalten von prochiralen Ketoestern unter den Bedingungen der Tietze-Allylierung. Gegenstand der Untersuchung war dabei zum einen die Abhängigkeit der Diastereoselektivität der Reaktion von der Anzahl der Methylengruppen zwischen Ketogruppe und Esterfunktion und zum anderen der Einfluss unterschiedlicher Alkoholreste an der Carboxylgruppe (s Tabelle 6-2).

Tabelle 6-2:Asymmetrische Allylierung von Ketoestern nach L. F. Tietze.

\begin{tabular}{|c|c|c|c|c|c|}
\hline & 87 & $\underset{(R)-60}{\mathrm{TMSO}} \overbrace{\mathrm{lh}}^{\mathrm{Ph}} \mathrm{Ph}$ & 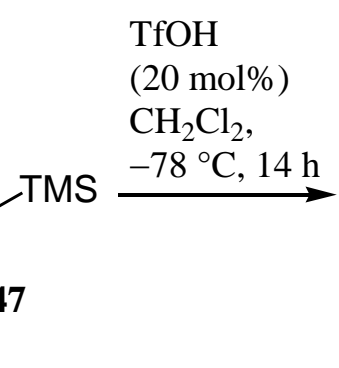 & $\underbrace{\mathrm{Ph}}_{\substack{\text { syn-(1'R)- } \\
\mathbf{8 8 - 9 0}}}$ & $\begin{array}{l}n t i-\left(1^{\prime} R\right)- \\
88-90\end{array}$ \\
\hline Nr. & & Ketoester & Homoallylether & Ausbeute [\%] & syn:anti \\
\hline$\overline{1}$ & 85 & $\mathrm{n}=1, \mathrm{R}=\mathrm{Me}$ & 88 & - & - \\
\hline 2 & $86 a$ & $\mathrm{n}=2, \mathrm{R}=\mathrm{Me}$ & $89 a$ & 91 & $94: 6$ \\
\hline 3 & $86 b$ & $\mathrm{n}=2, \mathrm{R}=i-\operatorname{Pr}$ & $89 b$ & 94 & $94: 6$ \\
\hline 4 & $86 c$ & $\mathrm{n}=2, \mathrm{R}=t-\mathrm{Bu}$ & $89 c$ & $66^{[\mathrm{a}]}$ & $93: 7$ \\
\hline 5 & 87 & $\mathrm{n}=3, \mathrm{R}=\mathrm{Me}$ & 90 & 98 & $91: 9$ \\
\hline
\end{tabular}

[a] Auxiliar rac-60 wurde verwendet. 
Stellvertretend für die Klasse der $\beta$-Ketoester wurde der kommerziell erhältliche Acetessigsäuremethylester (85) eingesetzt. Jedoch konnte unter den Reaktionsbedingungen der MCAR keine Bildung des Allylierungsproduktes 88 festgestellt werden. Ein möglicher Grund könnte in der Hemmung der Auxiliaranlagerung an die Ketogruppe aufgrund mangelnder Aktivierung liegen. Weiterhin besitzten $\beta$-Ketoester gegenüber anderen Ketonen eine erhöhte $\alpha-\mathrm{C}-\mathrm{H}-$ Acidität $\left(\mathrm{pK}_{\mathrm{a}}(\mathrm{DMSO})=14.2\right.$, vgl. $\mathrm{z} . \mathrm{B}$. Aceton: $\left.\mathrm{pK}_{\mathrm{a}(\mathrm{DMSO})}=26.5\right),{ }^{[44]}$ welche eine leichte Enolisierbarkeit zur Folge hat. Das durch Protonierung von 85 gebildete Oxeniumion 91 kann sich somit unter Abspaltung eines der $\alpha-\mathrm{C}-\mathrm{H}$-aciden Protonen in die durch Konjugation mit der Carboxylgruppe stabilisierte Enolform umwandeln. Enole wiederum sind deutlich bessere Nucleophile als Allylsilane, wodurch sich eine Allylierung der Ketogruppe von 85 wahrscheinlich ausschließt. Die mögliche Bildung von Aldolkondensationsprodukten von $\mathbf{8 5}$ mit sich selbst wurde in diesem Zusammenhang nicht weiter untersucht.<smiles>COC(=O)CC(C)=O</smiles>

85

(Ketoform)

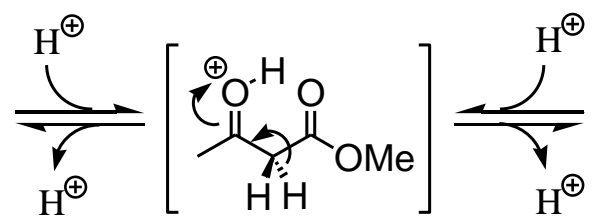

91

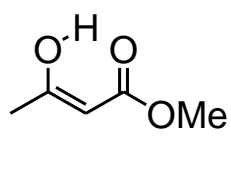

85

(Enolform)

Schema 6-7: Säurekatalysierte Keto-Enol-Tautomerie von Acetylessigsäuremethylester (85).

Durch Verlängerung der Kohlenstoffkette zwischen Carbonyl- und Carboxylfunktion um eine Methyleneinheit gelangt man zu den Lävulinsäureestern 86, welche zur Stoffklasse der $\gamma$-Ketoester gehören. Im Allgemeinen verlief die Allylierungsreaktion von 86 in sehr guten Ausbeuten von $>90 \%$. Eine Ausnahme bildet hierbei nur der tert-Butylester 86c, welcher lediglich mit einer Ausbeute von 66\% erhalten werden konnte. Die Allylierungsprodukte 89 zeigen unabhängig vom verwendeten Alkoholrest die gleiche hohe Diastereoselektivität von 94:6. Da eine vergleichbar hohe Selektivität bislang nur bei der Allylierung von $\alpha$-verzweigten Methylketonen beobachtet werden konnte, musste die Esterfunktionalität einen Nachbargruppeneffekt ausüben. Das intermediär gebildete Oxocarbeniumion 92 kann unter Ausbildung eines fünfgliedrigen Rings mit einem der freien Elektronenpaare (bzw. durch Wechselwirkung mit einem der nicht-bindenden Orbitale) des Carbonylsauerstoffs der Estergruppe stabilisiert werden. Damit wird ein Teil der positiven Ladung in die 
Carboxylfunktion transferiert, welche im Fall des tert-Butylesters 86c auch zur partiellen Abspaltung des tert-Butylcarbokations und damit zur Bildung des Nebenproduktes 94 in 12\% Ausbeute führt. Auffällig ist, dass 94 im Gegensatz zum Allylierungsprodukt 89c lediglich eine Diastereoselektivität von 73:27 aufweist (s. Schema 6-8).

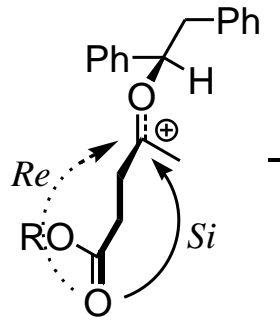

92

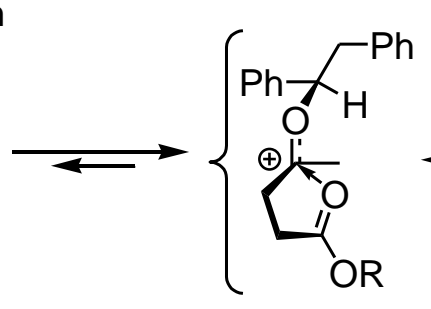

Si-93

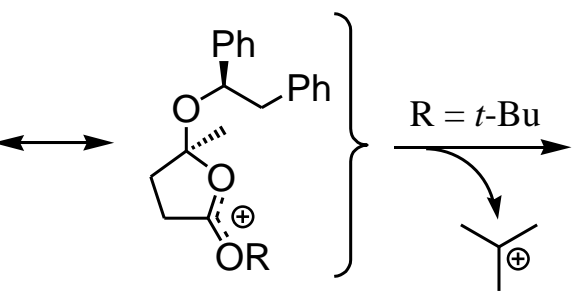<smiles>O=C1CCC(Cc2ccccc2)(OC(Cc2ccccc2)c2ccccc2)O1</smiles>

94 $d r: 73: 27$

Schema 6-8: $\quad$ Stabilisierung des Oxeniumions 92 durch die Carboxylfunktion und Bildung des Nebenproduktes 94 bei der Allylierung des tert-Butylesters 86c.

Mechanistisch sind für die Anlagerung des Allylsilans 47 sowohl die übliche $\mathrm{S}_{\mathrm{N}} 1$ artige Reaktion mit dem offenkettigen Oxeniumion 92 (s. Kapitel 4.2.2) als auch eine $\mathrm{S}_{\mathrm{N}} 2$-artige Reaktion mit den acetal-artigen Carbokationen Si-93 bzw. Re-93 denkbar. Zusätzlich eingesetzte DFT-Rechnungen zur Analyse von Grund- und Übergangszuständen hinsichtlich Geometrie und Energie lieferten keine Erkenntnisse hinsichtlich der Klärung dieses Phänomens.

Durch Erweiterung der Kettenlänge von 86a um eine weitere Methyleneinheit zwischen Carbonyl- und Carboxylfunktion erhält man den $\delta$-Ketoester 5-Oxohexansäuremethylester (87). Bei der Allylierungsreaktion von 87 wurde das Allylierungsprodukt 90 in einer exzellenten Ausbeute von 98\% und mit einer ebenfalls guten Diasteroselektivität von 91:9 erhalten. Im Vergleich zur Allylierung von 2-Heptanon (30b), welche eine leicht geringere Selektivität von 88:12 aufweist, muss auch diesmal - wenn auch in geringerem Maße als bei den Lävulinsäureestern - von einem Nachbargruppeneinfluss der Estergruppe ausgegangen werden. 


\subsubsection{Diskussion spektroskopischer Daten von Homoallylether 89a}

\subsubsection{1 ${ }^{1}$ H-NMR Spektroskopie}

Bei der Betrachtung des ${ }^{1}$ H-NMR-Spektrums des Homoallylethers 89a fallen zunächst die beiden intensiven Singuletts für die zwei Methylgruppen bei $\delta=0.82 \mathrm{ppm}$ und $3.63 \mathrm{ppm}$ auf. Anhand der charakteristischen Tieffeldverschiebung des Signals der zweiten Methylgruppe kann dieses zweifelsfrei der Methoxygruppe des Esters und erstere der aliphatischen Methylgruppe an C-4 zugeordnet werden.
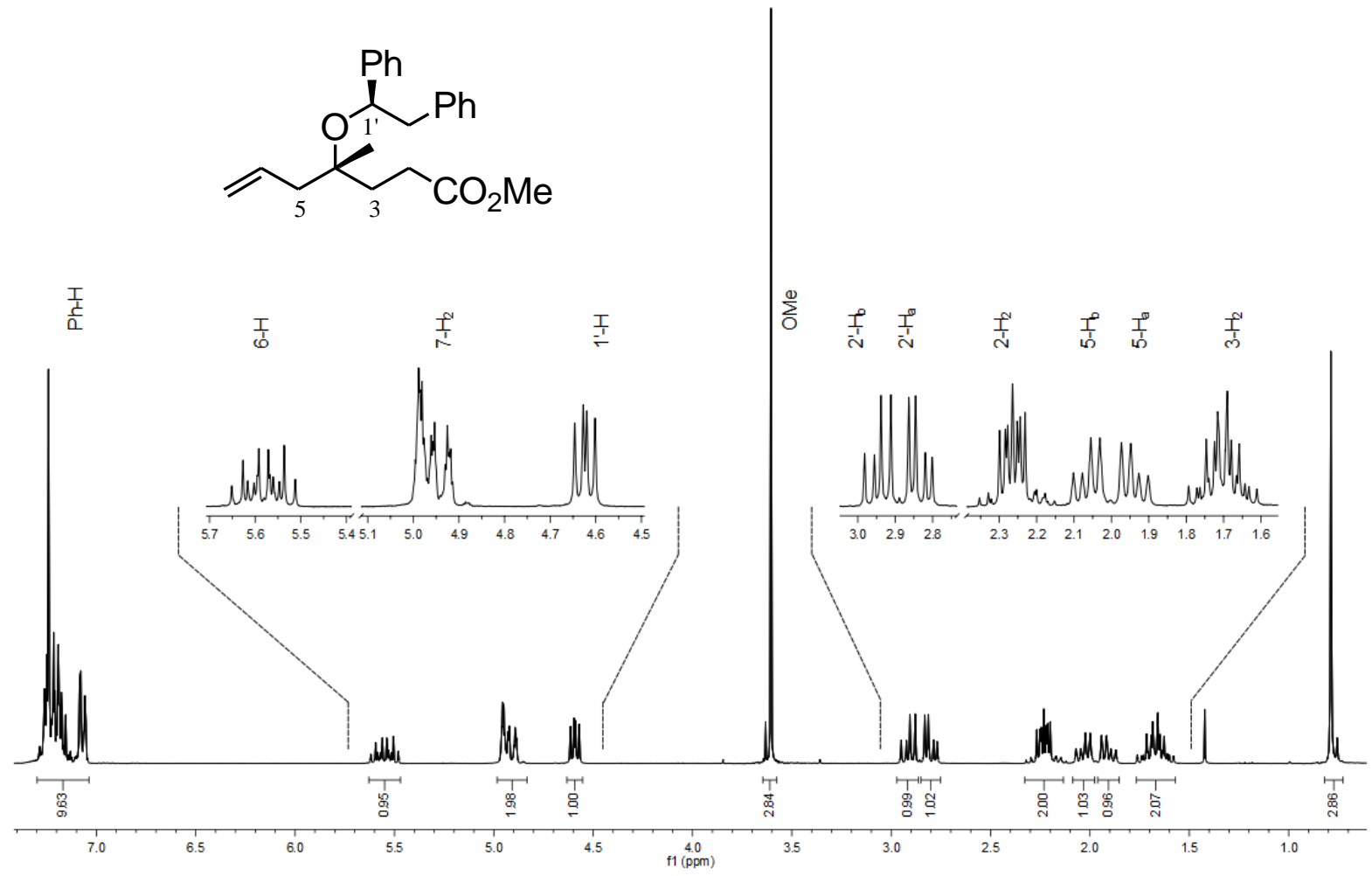

Abb. 6-3: ${ }^{1}$ H-NMR-Spektrum des Homoallylethers 89a.

Das Signal bei $\delta=5.58$ ppm entspricht der Resonanz von 6-H und kann anhand seines Kopplungsmuster zugeordnet werden. Die vier intensivsten Linien des Multipletts von 6-H bilden aufgrund der cis- und trans-Kopplung $\left({ }^{3} J_{\text {cis }}=10.4 \mathrm{~Hz},{ }^{3} J_{\text {trans }}=16.7 \mathrm{~Hz}\right) \mathrm{mit}$ den anderen Protonen der Doppelbindung an C-7 ein Dublett von Dublett, wobei jede Linie durch die vicinale Kopplung $\left({ }^{3} J=7.3 \mathrm{~Hz}\right)$ zur Methylengruppe $5-\mathrm{H}_{2}$ nochmals in ein Triplett aufgespalten wird. Die beiden diastereotopen Protonen an C-7 bilden aufgrund von Fernkopplungen zur Methylengruppe von C-5 bei $\delta=4.95 \mathrm{ppm}$ ein Multiplett. Als nächstes folgen die charakteristischen Signale für die aliphatischen 
Protonen der Auxiliargruppe (1'-H, 2'- $\left.\mathrm{H}_{2}\right)$. Das Proton 1'-H, welches bei $\delta=4.62 \mathrm{ppm}$ resoniert, ist aufgrund der unterschiedlichen vicinalen Kopplungskonstanten $\mathrm{zu}^{\prime}-\mathrm{H}_{\mathrm{a}}$ $\left({ }^{3} J=5.4 \mathrm{~Hz}\right)$ und $2^{\prime}-\mathrm{H}_{\mathrm{b}}\left({ }^{3} J=7.8 \mathrm{~Hz}\right)$ in ein Dublett von Dublett aufgespalten. Die diasterotopen Protonen der Methylengruppe von C-2' resonieren bei $\delta=2.83 \mathrm{ppm}$ $\left(2^{\prime}-\mathrm{H}_{\mathrm{a}}\right)$ und $\delta=2.95 \mathrm{ppm}\left(2^{\prime}-\mathrm{H}_{\mathrm{b}}\right)$ jeweils als Dublett von Dublett, welches sich aus der vicinalen Kopplung zu 1 '-H und der geminalen Kopplung $\left({ }^{2} J=13.2 \mathrm{~Hz}\right)$ der beiden Protonen untereinander ergibt. Auffällig dabei ist, dass die theoretischen Intensitätsverhältnisse der Einzelsignale (Dublett von Dublett: vier gleichintensive Linien) nicht beobachtet werden. Der Grad der Abschrägung eines Signals, welcher ebenfalls an den anderen Methylengruppen von C-2, C-3 und C-5 beobachtet werden kann, wird auch als Dacheffekt ${ }^{[45]}$ bezeichnet. Der Einfluss des Dacheffekts auf die Signalintensität ist immer dann stark, wenn der Abstand $\Delta v$ bzw. $\Delta \delta$ der koppelten Kerne klein im Verhältnis zur ihrer Kopplungskanstante $J$ ist. Die Zuordnung der einzelnen Methylengruppen zu den verbleibenden drei Signalen ergibt sich zum einen aus der bereits angesprochenen allylischen Fernkopplung, welche ebenfalls eine Verbreiterung des Signals der Protonen 5- $\mathrm{H}_{2}$ bewirkt und zum anderen aus der direkten Nachbarschaft

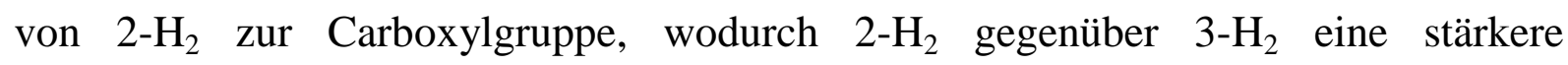
Entschirmung erfährt. Diese Zuordnung der Signale konnte auch mittels zweidimensionaler ${ }^{1} \mathrm{H}-{ }^{1} \mathrm{H}$-Korrelation (COSY) bestätigt werden. Während die Signale der allylischen Methylgruppe von C-5 als zwei verbreiterte Dubletts von Dublett erkennbar sind, überlagern die Resonazen der einzelnen, diastereotopen Protonen der Methylengruppen von C-2 und C-3 zu je einem Multiplett. Da die Signale des Minderdiasteromers anti-89a bis auf wenige Ausnahmen (in diesem Fall: Methyl- und Methoxygruppe) mit denen von syn-89a überlagert sind, wurde generell zur Bestimmung des Diastereomerenverhältnisses zusätzlich das ${ }^{13} \mathrm{C}-\mathrm{NMR}-$ Spektrum herangezogen. 


\subsubsection{2 ${ }^{13}$ C-NMR-Spektroskopie}

Das ${ }^{1}$ H-entkoppelte ${ }^{13} \mathrm{C}$-NMR-Spektrum des Homoallylethers 89a zeigt insgesamt 19 Signale, von denen sich acht im Bereich der Resonanz von aliphatischen Kohlenstoffen $(\delta<100 \mathrm{ppm})$ befinden. Die Zuordnung der einzelnen Signale wurde anhand von zweidimensionalen ${ }^{1} \mathrm{H}_{-}{ }^{13} \mathrm{C}$-Korrelationsspektren (HSQC, HMBC) durchgeführt.

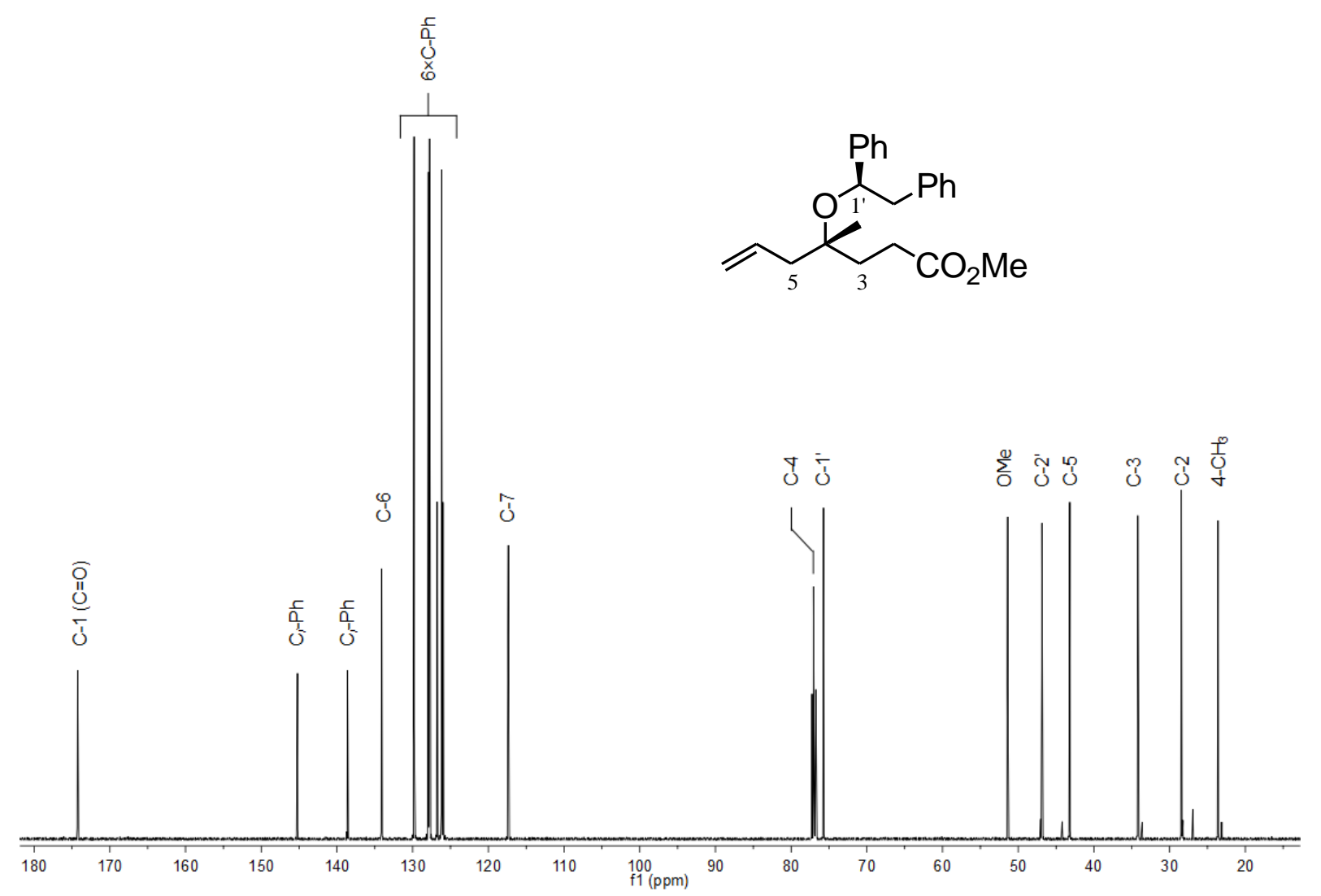

Abb. 6-4: ${ }^{13}$ C-NMR-Spektrum des Homoallylethers 89a.

Zur Diskussion des Spektrums werden zunächst die charakteristischen, chemischen Verschiebungen, der im Tieffeld resonierenden Kohlenstoffe herangezogen. Das am stärksten entschirmte Signal bei $\delta=174.3$ ppm entspricht der Resonanz des Carbonylkohlenstoff (C-1) der Estergruppe. Im Verschiebungsbereich der aromatischen bzw. ungesättigten Kohlenstoffe ( $\delta=100-160 \mathrm{ppm}$ ) erscheinen die Signale für die beiden Phenylreste der Auxiliargruppe und die der allylischen Doppelbindung. Die Signale der ortho- und meta-Kohlenstoffatome der beiden Phenylgruppen sind dabei aus Symmetriegründen doppelt so intensiv wie die Signale für die para-Kohlenstoffatome. Die quartären ipso-Kohlenstoffatome, bei $\delta=138.6 \mathrm{ppm}$ und $145.2 \mathrm{ppm}$, lassen sich auch aufgrund ihrer deutlich geringeren Intensität zuordnen. Im aliphatischen Bereich des Spektrums fallen zunächst die Signale der beiden Stereozentren (C-4, C-1') bei 
$\delta=77.1 \mathrm{ppm}$ und $\delta=75.7 \mathrm{ppm}$ auf. Während die restlichen Signale bei Verschiebungen von $\delta<55$ ppm anzutreffen sind, resoniert das Kohlenstoffatom C-1' aufgrund der Nachbarschaft zur Phenylgruppe ebenfalls im typischen Resonanzbereich von tertiären Alkoholen $(\delta=70-80$ ppm).

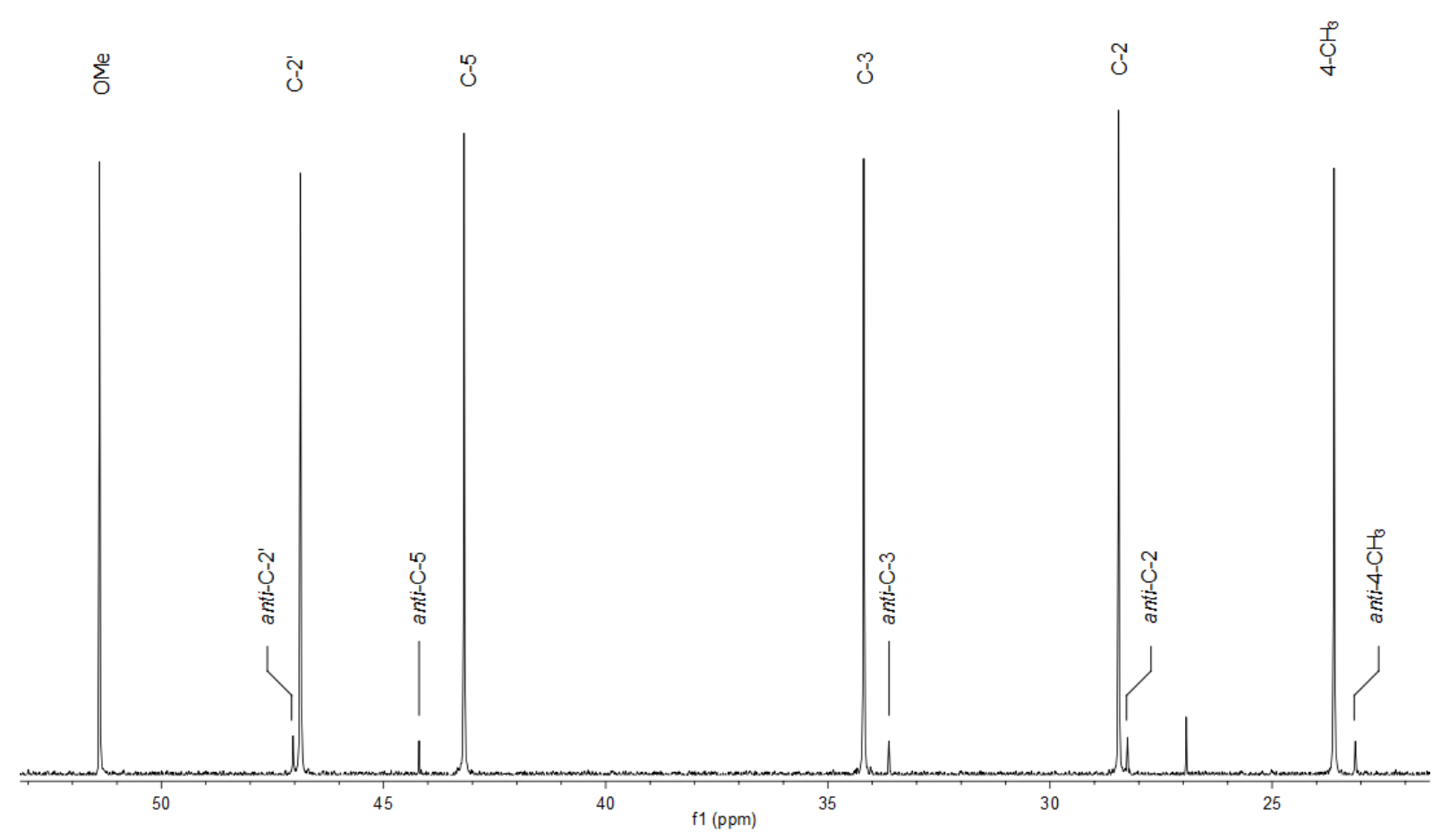

Abb. 6-5: Ausschnitt aus dem ${ }^{13}$ C-NMR-Spektrum $(\delta<55$ ppm) des Homoallylethers 89a.

Das Signal des Methoxyrestes bei $\delta=51.4$ ppm ist charakteristisch und liegt stets am unteren Ende des Verschiebungsbereichs für Alkohole $(\delta=50-80 \mathrm{ppm})$. Neben dem Kohlenstoffatom C-1' ( $\delta \approx 76 \mathrm{ppm})$ gehört auch C-2' zur Auxiliargruppe und resoniert ebenfalls bei einer für diese Gruppe typischen chemischen Verschiebung von $\delta \approx 47 \mathrm{ppm}$. Es folgen die Signale für die Methylengruppen C-5 und C-3, die Methylgruppe 4- $\mathrm{CH}_{3}$, sowie die Methylengruppe (C-2) in Nachbarschaft zur Carboxylgruppe. Während sich die Entschirmung von C-3 vorrangig aus der Nähe zum Strukurmotiv der tertiären Hydroxylgruppe ergibt, resoniert das Kohlenstoffatom der allylische Methylengruppe (C-5) aufgrund der zusätzlichen Entschirmung durch die Doppelbindung bei tieferem Feld. Des Weiteren lassen sich bei genauerer Betrachtung des Spektrums ebenfalls die einzelnen Signale des Minderdiastereomers anti-89a beobachten. Während sich die meisten Signale von syn- und anti- 
Diastereomer in ihrer chemischen Verschiebung kaum unterscheiden, treten die größten Abweichungen (bis zu $\Delta \delta=0.5-1.0 \mathrm{ppm}$ ) bei den Kohlenstoffkernen auf, die sich in direkter Nachbarschaft zum Stereozentrum C-4 befinden. Bei Fehlen vergleichbarer Signale im ${ }^{1}$ H-NMR-Spektrum, kann durch eine arithmetische Mittelung aller bestimmbaren syn/anti-Intensitätsverhältnisse im ${ }^{13} \mathrm{C}$-NMR-Spektrum näherungsweise das Diastereomerenverhältnis erhalten werden.

\subsubsection{Synthese von Pinnatolid und Furopinnatin}

Im Rahmen des Retrosyntheseweges B wurde zunächst die allylische Doppelbindung des in exzellenter Ausbeute und Selektivität erhaltenen Homoallylethers 89a oxidativ gespalten. Dazu wurde mit 89a bei $-78^{\circ} \mathrm{C}$ in Dichlormethan eine Ozonolyse durchgeführt. Durch Verwendung von Triphenylphosphin als Peroxidfänger konnte der Aldehyd 95 in einer sehr guten Ausbeuten von 98\% erhalten werden. Des Weiteren konnte das Rohprodukt der Ozonolyse auch direkt mit Natriumborhydrid in methanolischer Lösung in einer hervorragenden Gesamtausbeute von 98\% über beide Stufen zum entsprechenden Alkohol 96 umgesetzt werden (s. Schema 6-9).

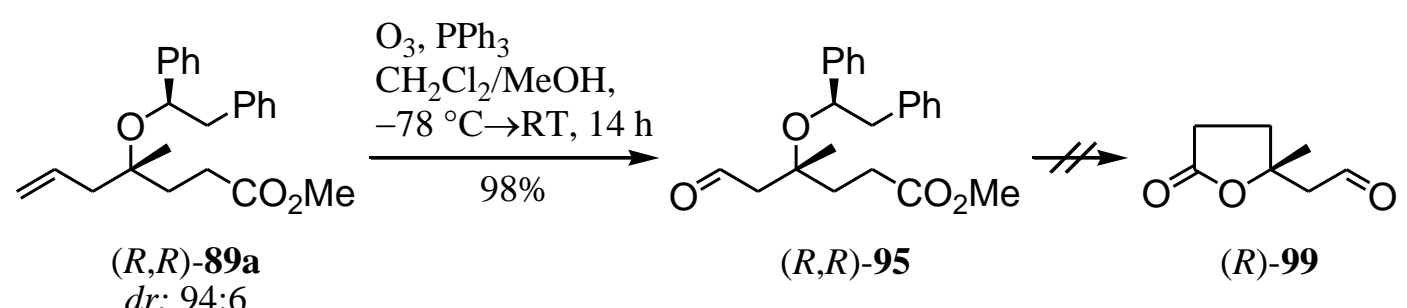

i) $\mathrm{O}_{3}, \mathrm{PPh}_{3}$

$\mathrm{CH}_{2} \mathrm{Cl}_{2} / \mathrm{MeOH}$,

$-78^{\circ} \mathrm{C} \rightarrow \mathrm{RT}, 14 \mathrm{~h} \quad 98 \%$

ii) $\mathrm{NaBH}_{4}$

$\mathrm{MeOH}, \mathrm{RT}, 0.5 \mathrm{~h}$

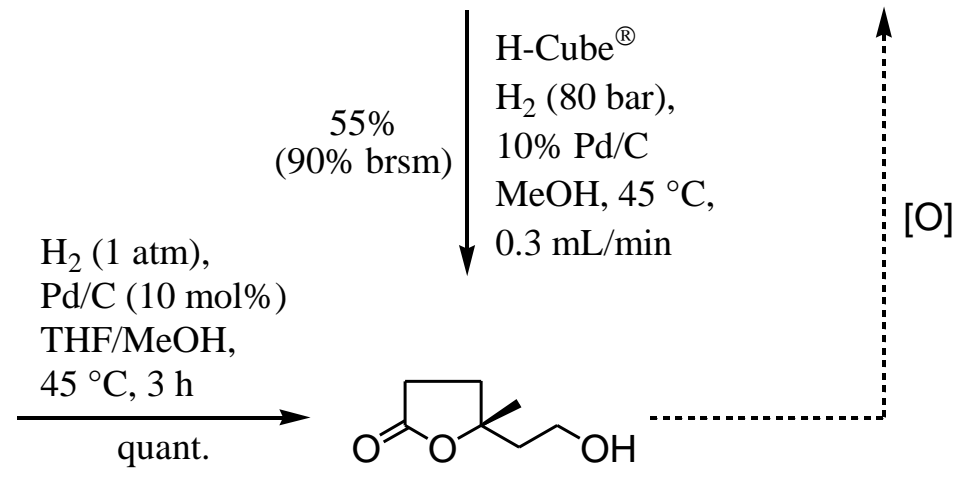

$(R, R)-96$

$(R)-98$

Schema 6-9: Oxidative Spaltung der Allylgruppe von 89a zum Aldehyd 95 oder alternativ zum Alkohol 96 und hydrogenolytische Abspaltung der Auxiliargruppe von 95 und 96. 
Die Hydrogenolyse zur Abspaltung der Auxililargruppe, welche $(R, R)-95$ in das $\gamma$-Lacton $(R)$-99 überführen sollte, wurde zunächst am Flussreaktor H-Cube ${ }^{\circledR}$ mit einer $10 \% \mathrm{Pd} / \mathrm{C}$-Kartusche bei einem Druck von 80 bar und bei einer Temperatur von $45^{\circ} \mathrm{C}$ mit einem Methanolfluss von $0.3 \mathrm{~mL} / \mathrm{min}$ durchgeführt. Jedoch wurde unter diesen Reaktionsbedingungen ebenfalls die Reduktion der Aldehydfunktion unter Bildung des alkoholischen $\gamma$-Lactons $(R)-98$ beobachtet. Da selbst nach mehreren Durchläufen des Produkt-Substrat-Gemisches die Ausbeute von 98 nicht über 55\% gesteigert werden konnte, musste von einer Vergiftung des Katalysators durch die Carbonylverbindung 95 ausgegangen werden. Eine hydrogenolytischen Abspaltung der Auxiliargruppe in Gegenwart einer Aldehydfunktion war demzufolge nicht möglich. Stattdessen wurde der durch Reduktion von 95 mit Natriumborhydrid leicht zugängliche Alkohol $(R, R)$-96 für die hydrogenolytische Entschützung verwendet. Dieser ließ sich unter einer Wasserstoffatmosphäre mit einer katalytischen Menge von 10\%-igem Palladium auf Aktivkohle in einem Lösungsmittelgemisch aus THF und Methanol bei $40{ }^{\circ} \mathrm{C}$ quantitativ in das $\gamma$-Lacton $(R)$-98 überführen. Zur Rückführung der Synthese auf die eigentliche Route über den Aldehyd $(R)$-99 musste die primäre Hydroxylgruppe von 98 wieder reoxidiert werden.

\subsubsection{Abtrennung des anti-Diastereomers durch Umkristallisation des Dinitrobenzoesäureester 97}

Zwar machte der synthetische Umweg über den Alkohol $(R, R)-96$ eine zusätzliche Stufe erforderlich, eröffnete aber zugleich eine gute Möglichkeit zur Abtrennung des anti-Diastereomers $\left(4 S, 1^{\prime} R\right)-96$. Für die Trennung der beiden epimeren Verbindungen wurde abermals die Umkristallisation von Dinitrobenzoesäureestern herangezogen, welches bereits bei der enantiomerenreinen Darstellung des Phenyl-benzyl-Auxiliars $(R)$-60 sehr erfolgreich eingesetzt worden war. Dazu wurde das Diastereomerengemisch von 96 mit 3,5-Dinitrobenzoylchlorid (DNBCl) in Gegenwart von Triethylamin als Base und einer katalytischen Menge DMAP in Dichlormethan bei Umgebungstemperatur mit 91\% Ausbeute zum Ester 97 umgesetzt. Nach Umkristallisation aus siedendem Essigester/n-Heptan wurde der Dinitrobenzoesäureester mit $82 \%$ als stereoisomerenreine Verbindung erhalten (s. Schema 6-10). 


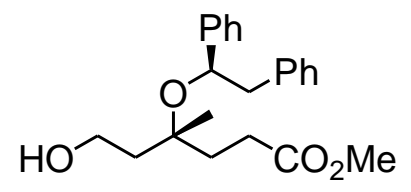

$(R, R)-96$

$$
d r: 94: 6 \mid>99: 1 \square
$$

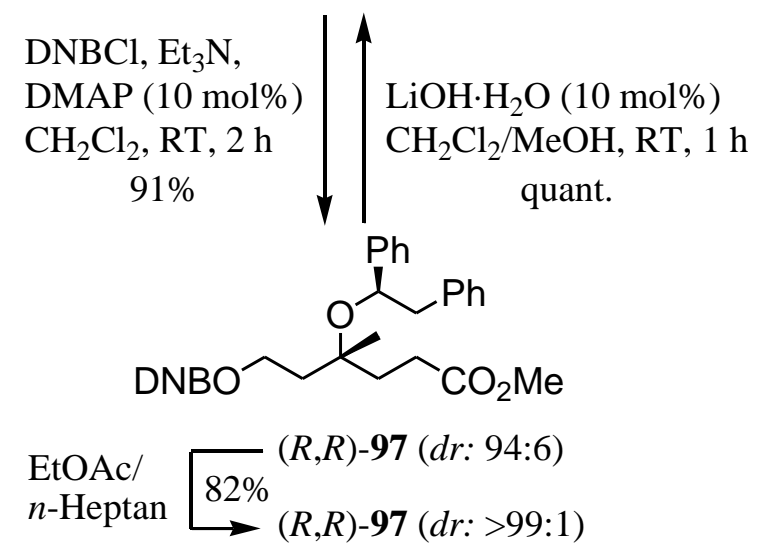

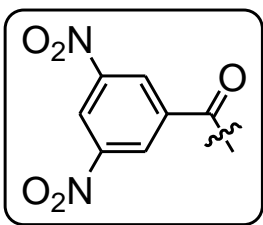

DNB

Schema 6-10: Verfahren zur Abtrennung des anti-Diastereomers von $(R, R)-96$ : Veresterung, Umkristallisation und Solvolyse des Dinitrobenzoesäureesters $(R, R)-97$.

Zur selektiven Spaltung des aromatischen Dinitrobenzoesäureesters in Gegenwart des aliphatischen Methylesters wurde eine basenkatalysierte Solvolyse durchgeführt. Diese lieferte in methanolischer Lösung mit katalytischen Mengen Lithiumhydroxid den Alkohol $(R, R)-96$ in quantitativer Ausbeute als einziges Stereoisomer. Damit ergibt sich für die preparative Diastereomerentrennung eine sehr gute Gesamtausbeute von $81 \%$ bezüglich der abtrennbaren Menge von $(R, R)-\mathbf{9 6}$ aus dem Epimerengemisch.

\subsubsection{Röntgenstrukturanalyse des Dinitrobenzoesäureester 97}

Zum zweifelsfreien Nachweis der vom allgemeinen Mechanismus der Allylierung nach Tietze postulierten $(R, R)$-Konfiguration für das Hauptepimer wurde in Zusammenarbeit mit dem Arbeitskreis Sheldrick von J. Holstein eine Röntgenstrukturanalyse von 97 angefertigt (s. Abb. 6-6). Die mit $\mathrm{Cu}-\mathrm{K}_{\alpha}$-Strahlung ( $\lambda=1.54 \AA$ ), im Stickstoffstrom bei $100 \mathrm{~K}$ aufgenommen Beugungsdaten zeigen, dass die Verbindung $(R, R)-97$ in der monoklinen Raumgruppe $\mathrm{P} 2_{1}$ mit einem monoklinen Winkel $\beta=111.56^{\circ}$ kristallisiert und die Elementarzelle $(a=6.82 \AA, b=27.01 \AA, c=7.80 \AA)$ zwei Formeleinheiten von 97 beinhaltet. Die Strukturverfeinerung der Beugungsdaten wurde mit dem Programm Shelx97 durchgeführt. Obwohl aufgrund der bekannten absoluten Konfiguration des eingesetzten Auxiliars $(R)-\mathbf{6 0}$ bereits eine einfache Bestimmung der relativen Konfiguration beider stereogener Zentren ausreichend 
gewesen wäre, konnte ebenfalls die absolute Konfiguration von 97 anhand der Summe der anormalen Dispersionsbeiträge der Sauerstoffatome nach der Parsons-Methode ${ }^{[46]}$ ermittelt werden. Dieses Verfahren ist eine Weiterentwicklung der konventionellen Flack-Methode $^{[47]}$. Ursprünglich war für eine zuverlässige enantiomorphe Bestimmung die Anwesenheit eines Schweratoms erforderlich, da die anormale Dispersion dann besonders stark ausgeprägt ist. Ausschlaggebend für die Bestimmung der absoluten Konfiguration von 97 nach Parsons war somit zum einen die Menge der Sauerstoffatome und zum anderen die Qualität des Kristalls, welche eine gute Verfeinerung der erhaltenen Beugungsdaten $(R$-Wert $=3.96 \%)$ erlaubte.

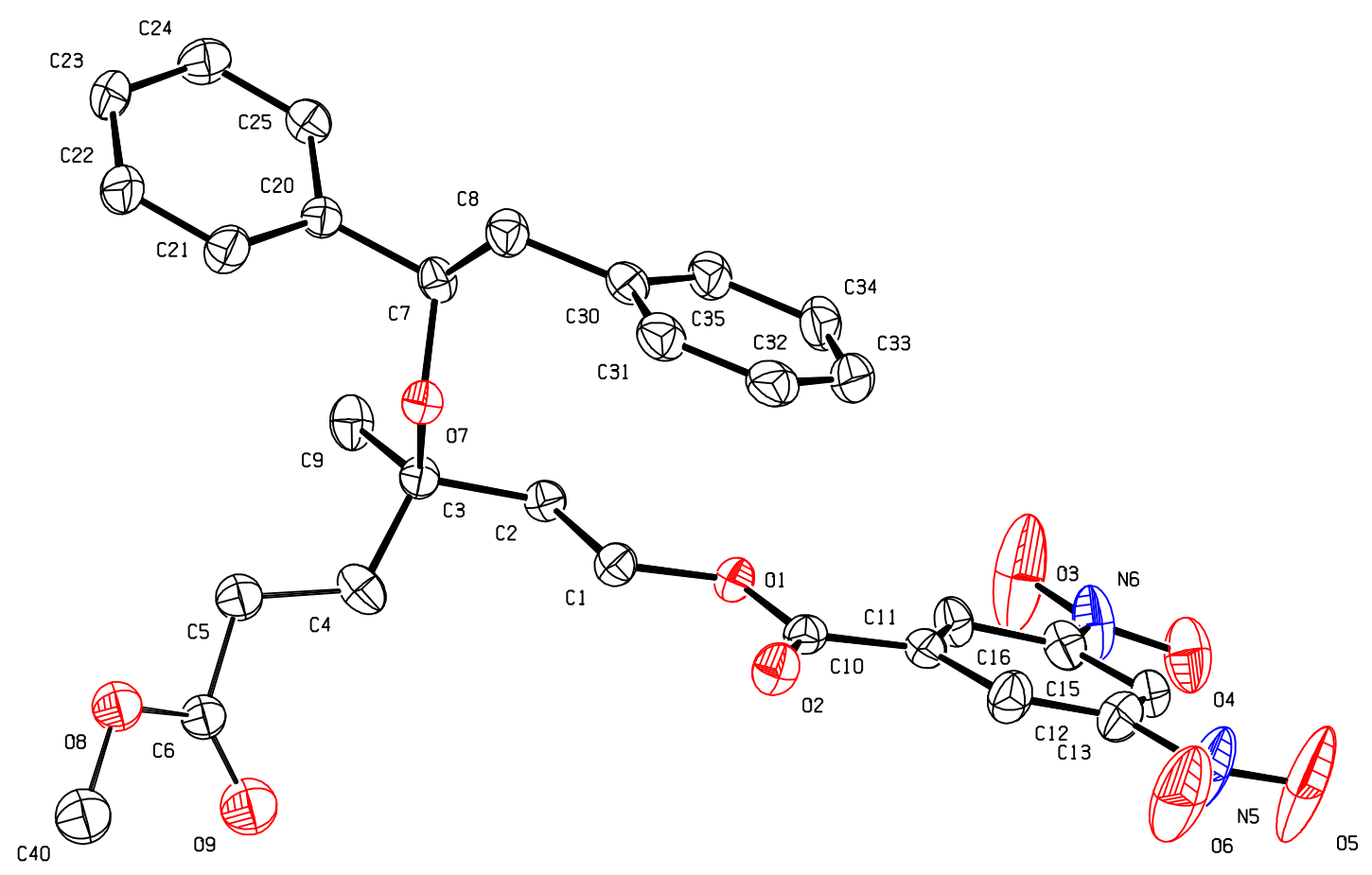

Abb. 6-6: Molekülstruktur von $(R, R)-97$ mit einem anisotropen Dislokalisationsparameter von 50\%. Abbildung wurde mit Platon gereniert. 


\subsubsection{Synthese von $(+)-(R)$-Pinnatolid}

Nach der Darstellung des epimerenreinen $(R, R)$-96 war das durch Palladiumkatalysierte Hydrogenolyse dargestellte $\gamma$-Lacton $(R)$-98 ebenfalls in enantiomerenreiner Form zugänglich (s. Schema 6-9). Im folgenden Schritt musste die primäre Hydroxylgruppe zur Aldehydfunktion reoxidiert werden. Diese Oxidation musste unter sehr milden Bedingungen - vor allem bezüglich des pH-Wertes - durchgeführt werden, weil der gebildete Aldehyd 99 leicht säure- oder basenkatalysiert ( $\mathrm{E}_{1}$ bzw. $\left.E_{1 \mathrm{cb}}\right)$ zum achiralen Enal 100 eliminieren konnte. Aus diesem Grund wurden die sehr milden Oxidationsverfahren Tetrapropylammoniumperruthenat(VII) (TPAP) ${ }^{[48]}$ und Dess-Martin-Periodinan $(\mathrm{DMP})^{[40]}$ als die am geeignetsten Methoden angesehen (s. Tabelle 6-3).

Tabelle 6-3: Reaktionsbedingungen zur milden Oxidation von $(R)-\mathbf{9 8}$.

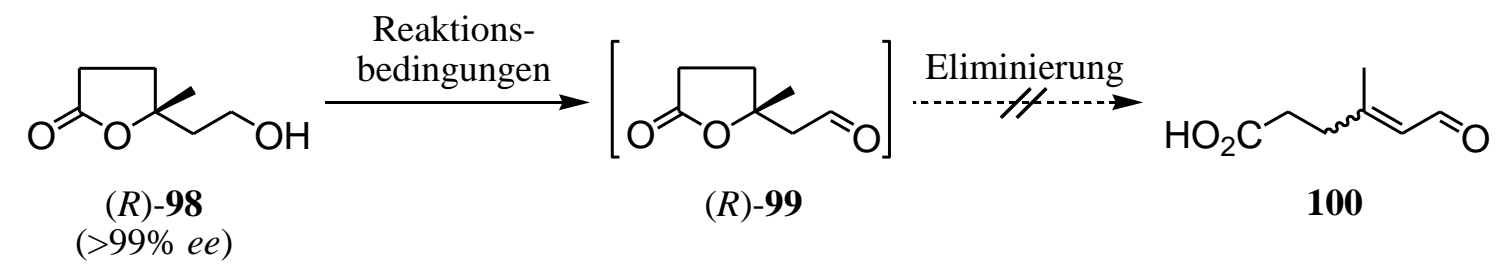

\begin{tabular}{c|l|l}
\hline Nr. & \multicolumn{1}{|c|}{ Reaktionsbedingungen } & \multicolumn{1}{c}{ Ergebnisse } \\
\hline \multirow{2}{*}{1} & $\begin{array}{l}n-\mathrm{Pr}_{4} \mathrm{~N}\left[\mathrm{RuO}_{4}\right](10 \mathrm{~mol} \%), \mathrm{NMO}(3.0 \mathrm{Äq} .) \\
\end{array}$ & $\begin{array}{l}\text { anteilige Eliminierung von 99 zu } \\
\mathbf{1 0 0} \text { unter den Reaktionsbed. }\end{array}$ \\
\hline \multirow{2}{*}{2} & $\begin{array}{l}\mathrm{DMP}(1.2 \mathrm{Aq} .) \\
\mathrm{CH}_{2}, \mathrm{RT}, 30 \mathrm{~min}\end{array}$ & $\mathbf{9 9}$ stabil unter Reaktionsbed. \\
& & $\begin{array}{l}{ }^{\circ} \mathrm{C} \rightarrow \mathrm{RT}, 30 \mathrm{~min} \\
\text { aber teilweise Eliminierung bei }\end{array}$ \\
\hline
\end{tabular}

Bei der Reaktion von $\mathbf{9 8}$ mit katalytischen Mengen am Ruthenium(VII)-Katalysator und $N$-Methylmorpholinoxid (NMO) als Reoxidant in Dichlormethan konnte neben der Bildung des Aldehyds $\mathbf{9 9}$ auch die Bildung einer deutlich polareren, UV-aktiven Verbindung beobachtet werden, bei welcher es sich um das unerwünschte Enal 100 handelt. Dagegen zeigte die Oxidation von 98 mit dem hypervalenten Iodreagenz DMP in Dichlormethan eine sehr saubere Umsetzung zum gewünschten Aldehyd 99, ohne das dabei das Eliminierungsprodukt auftrat. Bei der notwendigen Filtration der Reaktionslösung über Kieselgel zur Abtrennung der gebildeten Essigsäure, sowie den 
reduzierten Rückständen des DMP-Reagenz konnte bereits eine teilweise Zersetzung festgestellt werden. Aufgrund seiner Instabilität wurde der Aldehyd 99 direkt im Anschluss an seine Isolierung in THF mit dem Vinylgrignard 101 bei $-60{ }^{\circ} \mathrm{C}$ umgesetzt. Dabei konnte der Allylalkohol (5R)-71 mit einer mäßigen Ausbeute von 40\% erhalten werden. Die Diastereoselektivät der Addition wurde zu etwa 1:1.1 bestimmt. Für den Fortgang der Synthese war die Selektivität allerdings nicht weiter von Interesse, da das gebildete neue Stereozentrum in der finalen Oxidation wieder zerstört würde. Basierend auf den Erfahrungen der Oxidation von $(R)-\mathbf{9 8}$ wurde das Epimerengemisch von (5R)-71 ebenfalls mit dem DMP-Reagenz in einer Ausbeute von $90 \%$ zum (+)-(R)-Pinnatolid (25) umgesetzt.

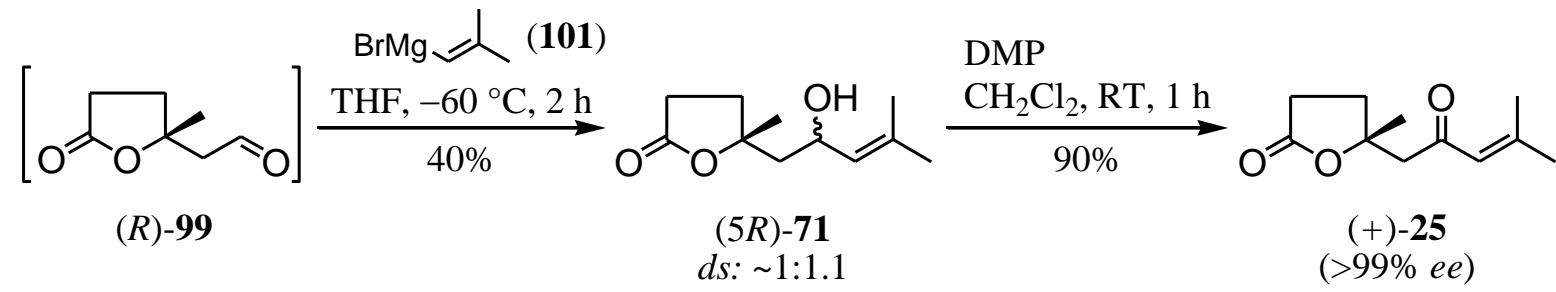

Schema 6-11: Grignard-Addition an (R)-99 und finale Oxidation zu (+)-Pinnatolid (25).

Die analytische Trennung der Enantiomeren von rac-25 zur Bestimmung des Enantiomerenüberschusses konnte mittels HPLC an der chiralen Phase Chiralpak ${ }^{\circledR}$ IB mit dem isokratischen Eluenten $n$-Hexan/ $i-\mathrm{PrOH}$ 85:15 und einem Fluss von $0.6 \mathrm{~mL} / \mathrm{min}$ erreicht werden.
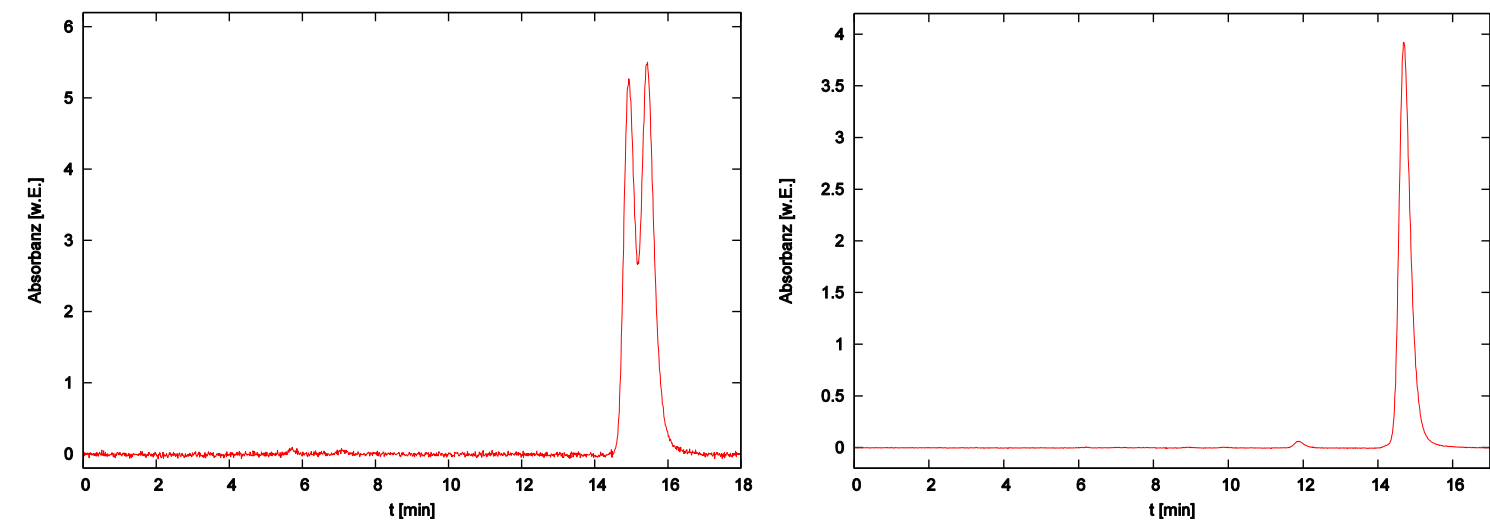

Abb. 6-7: HPLC-Chromatogramme von rac-25(links) und (R)-25 (rechts). 


\subsubsection{Diskussion spektroskopischer Daten von Pinnatolid}

\subsubsection{1 ${ }^{1}$ H-NMR-Spektroskopie}
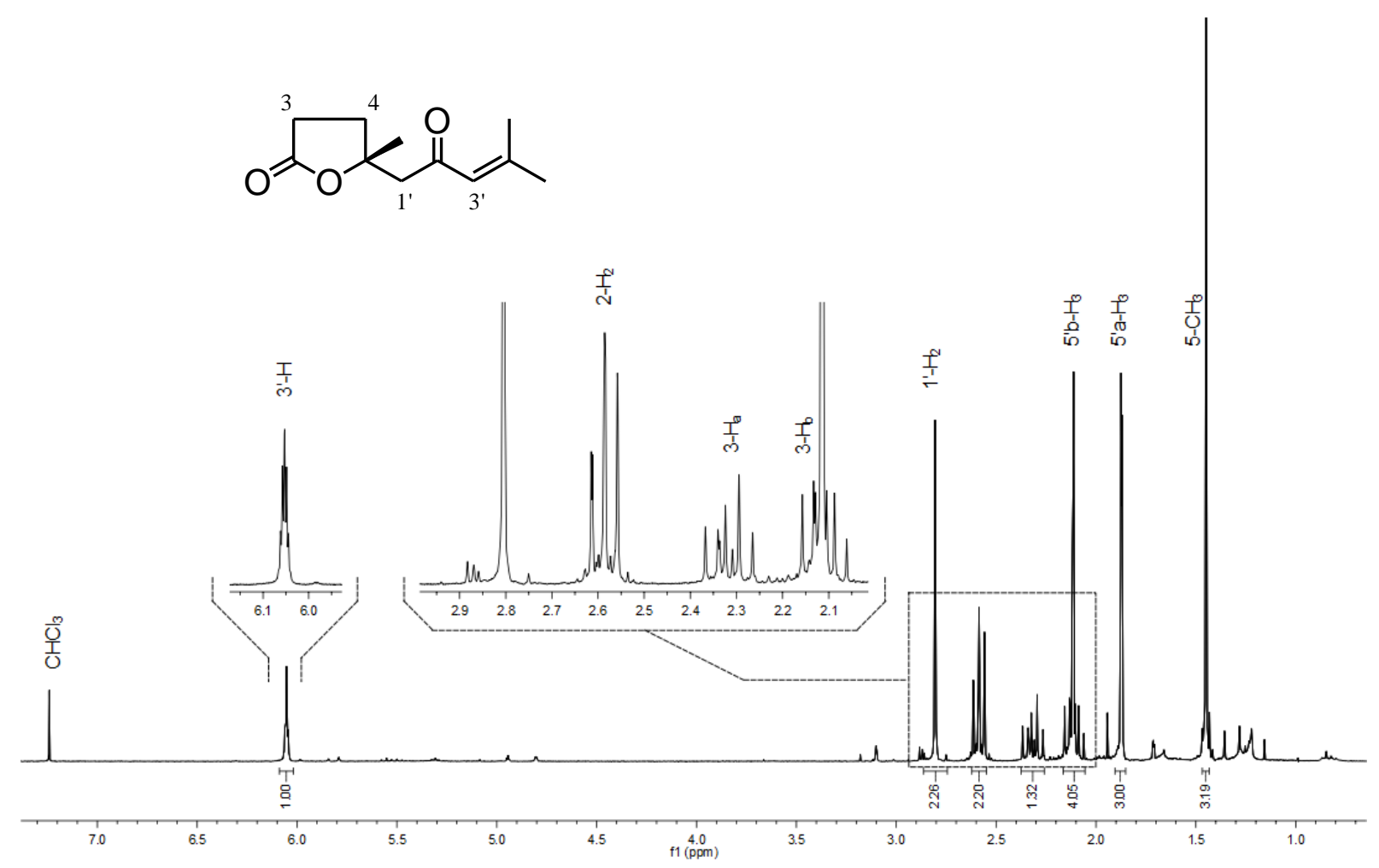

Abb. 6-8: ${ }^{1}$ H-NMR-Spektrum von Pinnatolid (25).

Im ${ }^{1}$ H-NMR-Spektrum von Pinnatolid (25) befinden sich, bis auf das Signal bei $\delta \approx 6.0 \mathrm{ppm}$, alle Signale im aliphatischen Bereich des Spektrums ( $\delta \approx 0-4 \mathrm{ppm})$. Die Protonen der isolierten Methylgruppe an C-5 resonieren bei $\delta=1.45 \mathrm{ppm}$ als Singulett. Aufgrund der Nachbarschaft zum konjugierten Enonsystem erscheinen die Signale der Protonen der beiden diastereotopen Methylgruppen (C-5'a, C-5'b) im Spektrum bei $\delta=1.87 \mathrm{ppm}$ und $\delta=2.12 \mathrm{ppm}$. Diese Signale spalten aufgrund der Fernkopplung zu 3'-H jeweils in ein Dublett mit einer Kopplungskonstante von ${ }^{4} J=1.2 \mathrm{~Hz}$ auf. Der Kopplungspartner, das Proton $3^{\prime}-\mathrm{H}$, resoniert in dem für Doppelbindungen charakteristischen Bereich bei $\delta=6.06 \mathrm{ppm}$ als ein Septett mit der analogen Kopplungskonstante. Die äußersten Linien des Septetts sind aufgrund der kleinen Kopplungskonstante und der Basisverbreiterung des Signals nur als Schultern zu erahnen. Die Signale der Protonen der Methylengruppe an C-1' $(\delta \approx 2.8 \mathrm{ppm})$ erscheinen als Singulett mit kleinen Sateliten. Da beide Protonen bezüglich des 
Stereozentrums diastereotop sind, sollte dieses Signal als zwei überlagerte Dubletts mit einer geminalen Kopplungskonstante von ${ }^{2} J=16.5 \mathrm{~Hz}$ und einem extrem ausgeprägten Dacheffekt gedeutet werden.

Abb. 6-9: Vergrößerter Ausschnitt aus dem ${ }^{1}$ H-NMR-Spektrum von Pinnatolid (25).

Die verbleibenden Signale entsprechen den Resonanzen der beiden Methylengruppen des Lactonringes (C-3, C-4), welche zusammen ein abgeschlossenes Spinsystem bilden. Die diastereotopen Protonen $4-\mathrm{H}_{\mathrm{a}}$ und $4-\mathrm{H}_{\mathrm{b}}$ resonieren jeweils als ein Dublett vom Triplett mit je einer geminalen und einer vicinalen Kopplungskonstante von ${ }^{2} J=13.1 \mathrm{~Hz}$ und ${ }^{3} J=7.9 \mathrm{~Hz}$ bzw. ${ }^{3} J=8.8 \mathrm{~Hz}$. Die Signale der Protonen von C-3, welche aufgrund der Nachbarschaft zum Carbonylkohlenstoff des Lactons weiter ins Tieffeld verschoben sind als die an C-4, erscheinen im Spektrum lediglich als ein gemitteltes Triplett mit einer Kopplungskonstante von ${ }^{3} J=8.4 \mathrm{~Hz}$. 


\subsubsection{2 ${ }^{13}$ C-NMR-Spektroskopie}
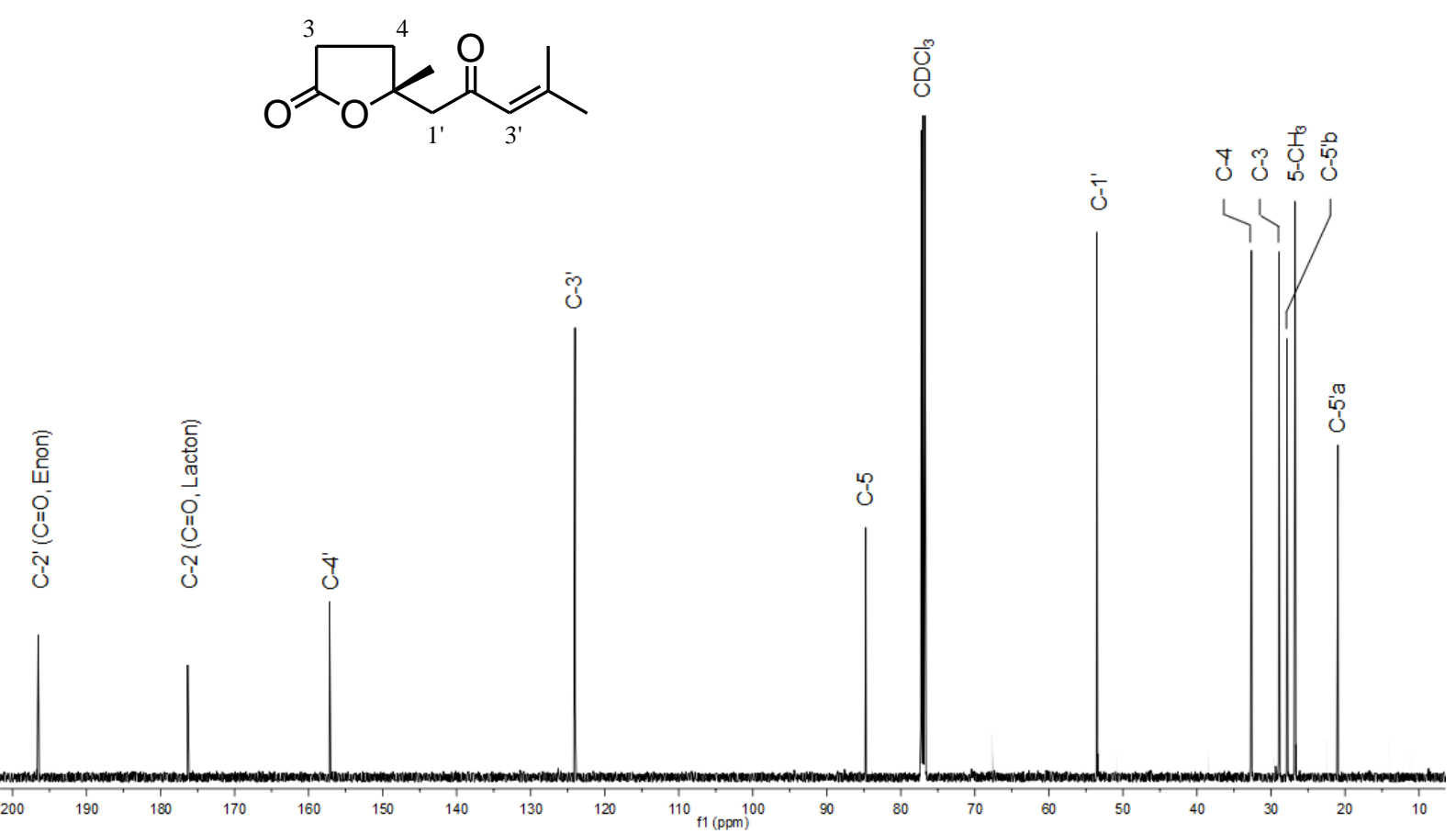

Abb. 6-10: ${ }^{13} \mathrm{C}-\mathrm{NMR}-$ Spektrum von Pinnatolid (25).

Im ${ }^{1}$ H-entkoppelten ${ }^{13} \mathrm{C}$-NMR-Spektrum von Pinnatolid (25) sind die Signale aller elf Kohlenstoffe $\mathrm{zu}$ erkennen, welche durch zweidimensionale ${ }^{1} \mathrm{H}-{ }^{13} \mathrm{C}-$ Korrelationen (HSQC, HMBC) zugeordnet worden sind. Bei der Betrachtung des Spektrums fällt auf, dass sich lediglich vier Signale bei einer chemischen Verschiebung von $\delta \geq 100$ ppm befinden, während der Großteil im aliphatischen Bereich des Spektrums $(\delta<100 \mathrm{ppm})$ liegt. Die am weitesten ins Tieffeld verschobenen Signale sind die Resonanzen des Carbonylkohlenstoffs der Ketogruppe bei $\delta=196.5 \mathrm{ppm}$ und des Carboxylkohlenstoffs des Lactons bei $\delta=176.4 \mathrm{ppm}$. Die Kohlenstoffe der Doppelbindung (C-3' und $\left.\mathrm{C}^{\prime} 4^{\prime}\right)$ resonieren bei $\delta=124.0 \mathrm{ppm}$ und $\delta=157.2 \mathrm{ppm}$. Charakteristisch ist dabei zum einen die für Enone typische starke Entschirmung der $\beta$-Position (C-4'), welche die für Doppelbindungen vergleichsweise hohe Verschiebung von $\delta=157.2 \mathrm{ppm}$ bewirkt, und zum anderen die deutlich geringere Intensität des quartären Kohlenstoffatoms C-4' gegenüber den tertiären C-3'. Im aliphatischen Bereich des Spektrums $(\delta<100 \mathrm{ppm})$ fallen aufgrund ihrer isolierten Lage vor allem die Signale bei $\delta=53.5 \mathrm{ppm}$ und $\delta=84.8 \mathrm{ppm}$ auf. Anhand des 
Intensitätsunterschiedes lässt sich die Resonanz bei $\delta \approx 85 \mathrm{ppm}$ dem verbleibenden quartären Kohlenstoff C-5 zuordnen. Die vergleichsweise hohe chemische Verschiebung von C-5 (üblicherweise $\delta=70-80 \mathrm{ppm}$ ), beruht auf der zusätzlichen Entschirmung durch die Carboxylgruppe des Lactons (vgl. tert-Butanol $\delta\left(C\left(\mathrm{CH}_{3}\right)_{3}\right)=69.6 \mathrm{ppm}$, Essigsäure-tert-butylester: $\left.\delta\left(C\left(\mathrm{CH}_{3}\right)_{3}\right)=79.4 \mathrm{ppm}^{[49]}\right)$. Die vergleichsweise starke Entschirmung von C-1' wird durch einen synergistischen Effekt aus der Nachbarschaft zu einem Carbonylkohlenstoff und zum Strukturmotiv des tertiären Alkohols verursacht. Die Einzelwirkung dieser Gruppen lassen sich für die Methylengruppen C-3 bei $\delta=28.9$ ppm neben der Carbonylfunktion und C-4 bei $\delta=32.6 \mathrm{ppm}$ neben dem tertiären Alkoholmotiv erkennen. Die verbleibenden drei Signale bei $\delta<28$ ppm entsprechen den Methylgruppen C-5'a, C-5'b und 5- $\mathrm{CH}_{3}$.

\subsubsection{CD-Spektroskopie}

Die CD-Spektroskopie gehört zu den chiroptischen Eigenschaften und beruht auf der unterschiedlichen Absorptionskraft einer chiralen Substanz für rechts- und linkszirkular polarisiertes Licht. Linear polarisiertes Licht, welches als Überlagerung dieser beiden zirkular polarisierten Lichtwellen (ohne Phasenverschiebung) angesehen werden kann, wird beim Durchgang durch ein chirales Medium in eine elliptisch polarisierte Welle umgewandelt. Die beobachtete Größe bei der CD-Spektroskopie ist somit die Elliptizität $\theta(\lambda)$ als Funktion der Wellenlänge $\lambda$. Die molare Elliptizität $[\theta]$, welche $\theta$ normiert auf die Konzentration $c$ und die Länge der Küvette $l$ entspricht, ist dabei direkt proportional zum Unterschied der molaren Absorptionskoeffizienten $\Delta \varepsilon$ für rechts- und linkszirkular polarisiertes Licht (s. Gl. (1)).

$$
[\theta]=\frac{\theta}{c l} \approx\left(\frac{\ln 10}{4}\right)\left(\frac{180}{\pi}\right) \Delta \varepsilon \approx 32.98 \Delta \varepsilon
$$

Anhand dieses Zusammenhangs wurden die für $(R)$-Pinnatolid (25) beobachteten Elliptizitäten $\theta(\lambda)$ direkt als $\Delta \varepsilon(\lambda)$ in Abhängigkeit der Wellenlänge aufgetragen. Das Absorptionsspektrum (UV/Vis) von $(R)-25$ wurde als Absorbanz (- $\left.\lg \left(I / I_{0}\right)\right)$ parallel (2. Kanal) zur Elliptizität aufgezeichnet. 


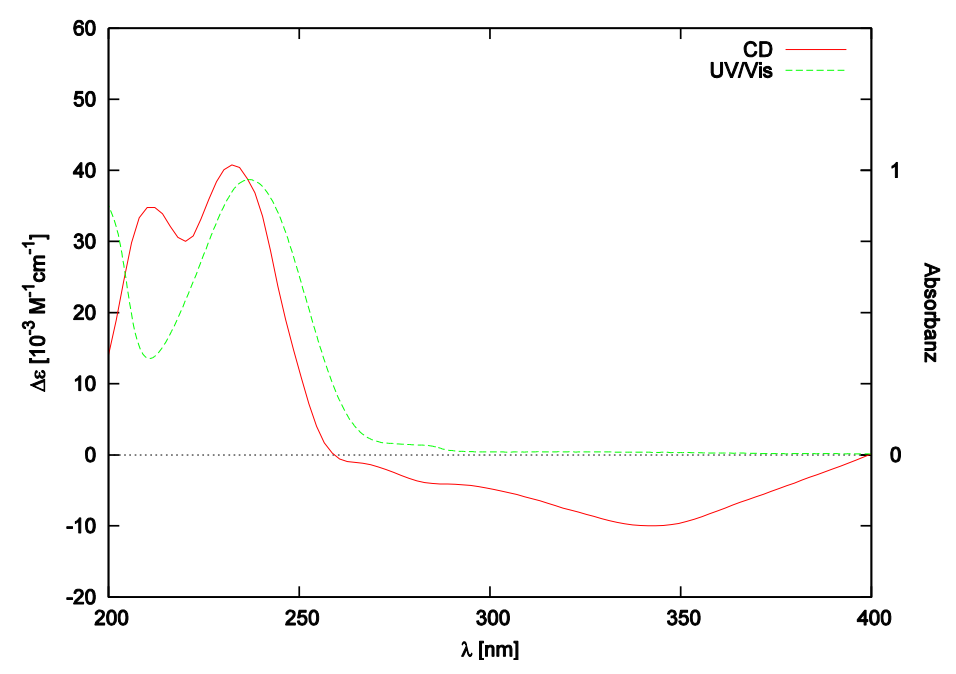

Abb. 6-11: CD- und UV/Vis-Spektrum von (+)-(R)-Pinnatolid (25).

Die Auftragung sowie die Glättung des stark verrauschten CD-Signals wurden mit dem Programm gnuplot durchgeführt. Da die Verbindung $(R)-\mathbf{2 5}$ im Bereich von $\lambda=300-400 \mathrm{~nm}$ keine Absorption aufweist, sollte die Elliptizität Null bzw. konstant und auf Null korrigierbar sein. Die ungewöhnliche Veränderung der Elliptizität bei $\lambda \approx 350 \mathrm{~nm}$ machte ein Erkennen der eigentlichen Nulllage nahezu unmöglich. Da aber im Bereich des $\pi \rightarrow \pi^{*}$-Übergangs $(\lambda=237 \mathrm{~nm})$ aufgrund des Cotton-Effekts ein Vorzeichenwechsel der Elliptizität zu erwarten ist, wurde die Kurve im Bereich von $\lambda>300 \mathrm{~nm}$ nach $\Delta \varepsilon<0$ korrigiert. 


\subsubsection{Untersuchungen zur Synthese von $(R)$-Furopinnatin}

Analog zur Retrosynthese sollte Furopinnatin (24) durch eine Addition einer 3-Furylmetall-Spezies an das $\gamma$-Lacton $\mathbf{7 1}$ unter Öffnung des Lactonrings gebildet werden. Die Addition von 3-Furyllithium an ein $\gamma$-Lacton wurde in der Literatur bereits einige Male mit fast durchgängig moderaten Ausbeuten zwischen 20-40\% beschrieben. ${ }^{[50 a]}$ Dennoch konnte die Gruppe um Vassilikogiannakis bei dieser Additionsreaktion eine sehr gute Ausbeute von $85 \%$ erreichen ${ }^{[50 b]}$. Die FuryllithiumSpezies wurde dabei in situ durch eine Lithiierung von 3-Bromfuran (103) mit $n$-BuLi hergestellt. Aufgrund eines stetigen Zersetzungsprozesses von 3-Bromfuran selbst bei $-18{ }^{\circ} \mathrm{C}$ im Gefrierschrank, war vor Gebrauch stets eine Destillation erforderlich. Erste Versuche mit $\gamma$-Butyrolacton (107, GBL) als Modellverbindung für das $\gamma$-Lacton 71 scheiterten unter den von Vassilikogiannakis berichteten Reaktionsbedingungen. Da sich GBL (107) aus rein praktischen Gesichtspunkten, wie leichte Flüchtigkeit und Schwierigkeiten bei der Reaktionkontrolle (Anfärbarkeit, UV-Aktivität) nur bedingt für die Optimierung von Reaktionsbedingungen eignete, wurde statt dessen das leicht zugängliche $\varepsilon$-Lacton 102 verwendet. Dazu wurde der racemische Alkohol 96 mit katalytischen Mengen $p$-Toluolsulfonsäure in Chloroform bei $60^{\circ} \mathrm{C}$ über $3 \mathrm{~h}$ in quantitativer Ausbeute zu rac-102 lactonisiert. Die so erhaltene Modellverbindung wurde für die Optimierung der Reaktionsbedingungen der Furyladdition eingesetzt. Trotz Verwendung einer vorgekühlten Transferkanüle für die Überführung der in situ gebildeten 3-Furyllithium-Lösung konnten keine reproduzierbaren Ergebnisse erhalten werden. Versuche zur Ummetallierung von 3-Furyllithium mit $\mathrm{MgBr}_{2}$ oder $\mathrm{ZnCl}_{2}$ waren ebenfalls nicht erfolgreich. Erst die direkte Zugabe einer Lösung des $\varepsilon$-Lactons 102 zur 3-Furyllithium-Lösung bei $-100^{\circ} \mathrm{C}$ und anschließenden langsamen Erwärmen auf $-60{ }^{\circ} \mathrm{C}$ lieferte das Acylfuran 104 in einer reproduzierbaren Ausbeute von 46\%. 
Tabelle 6-4: Synthese des $\varepsilon$-Lactons 102 und Optimierung der Reaktionsbedingungen für die Additon einer 3-Furylmetallverbindung an 102.

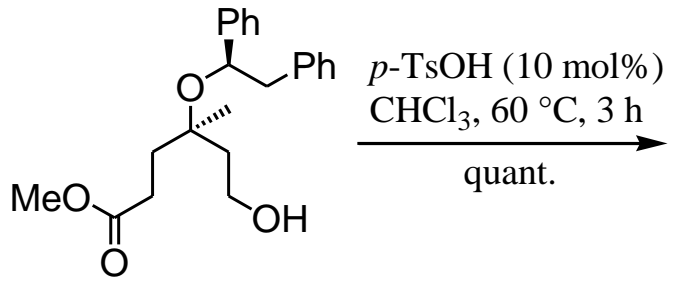

$\operatorname{rac}-(R, R)-96$

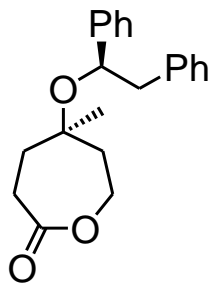

$\operatorname{rac}-(R, R)-102$

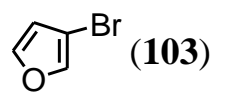

i) $103, n-\mathrm{BuLi}$ $-78^{\circ} \mathrm{C}, 15 \mathrm{~min}$

ii) Reaktionsbedingungen<smiles>C[C@](CCO)(CCC(=O)c1ccoc1)O[C@@H](Cc1ccccc1)c1ccccc1</smiles>

$\operatorname{rac}-(R, R)-104$

\begin{tabular}{c|l|c}
\hline Nr. & Reaktionsbedingungen & Ausbeute \\
\hline 1 & $\mathbf{1 0 2}$ (invers), THF, $-78 \rightarrow-60^{\circ} \mathrm{C}, 20 \mathrm{~min}$ & kein Umsatz \\
\hline 2 & $\begin{array}{l}\text { a) } \mathrm{MgBr}_{2}(2.3 \mathrm{Aq} .), \mathrm{THF},-78{ }^{\circ} \mathrm{C}, 0.5 \mathrm{~h} \\
\text { b) } \mathbf{1 0 2} \text { (invers), THF, }-60{ }^{\circ} \mathrm{C}, 2 \mathrm{~h}\end{array}$ & kein Umsatz \\
\hline 3 & $\begin{array}{l}\text { a) } \mathrm{ZnCl}_{2}\left(2.3 \ddot{\mathrm{Aq}} \text { ) }, \mathrm{THF},-78{ }^{\circ} \mathrm{C}, 0.5 \mathrm{~h}\right. \\
\text { b) } \mathbf{1 0 2} \text { (invers), } \mathrm{THF},-60{ }^{\circ} \mathrm{C}, 2 \mathrm{~h}\end{array}$ & \\
\hline 4 & $\mathbf{1 0 2}$ (direkt), THF, $-100 \rightarrow-60{ }^{\circ} \mathrm{C}, 2 \mathrm{~h}$ & $46 \%$ \\
\hline
\end{tabular}

Während die zweifache Additionsreaktion - also die unerwünschte Addition an das Acylfuranprodukt - bei der Reaktion mit dem $\varepsilon$-Lacton 102 weitgehend $(<5 \%)$ unterdrückt werden konnte, führte die Übertragung der optimierten Reaktionsbedingungen auf die Lactonöffnung von GBL (107) in 40\% Ausbeute zur Bildung eines Gemisches bestehend aus dem Acylfuran 108 und dem unerwünschten doppelten Additionsprodukt 109 im Verhältnis von ungefähr 1:1. Aufgrund dieses Ergebnisses konnte bereits erwartet werden, dass die Reaktion von (5R)-71 keine guten Ausbeuten ergeben würde. Dennoch wurde die Umsetzung unter den optimierten Reaktionsbedingungen durchgeführt. Jedoch trotz Einsatzes eines zusätzlichen Äquivalentes „3-Furyllithium“ zwecks Deprotonierung der freien Hydroxylgruppe konnte auch nach mehreren Versuchen keine Umsetzung festgestellt werden. 

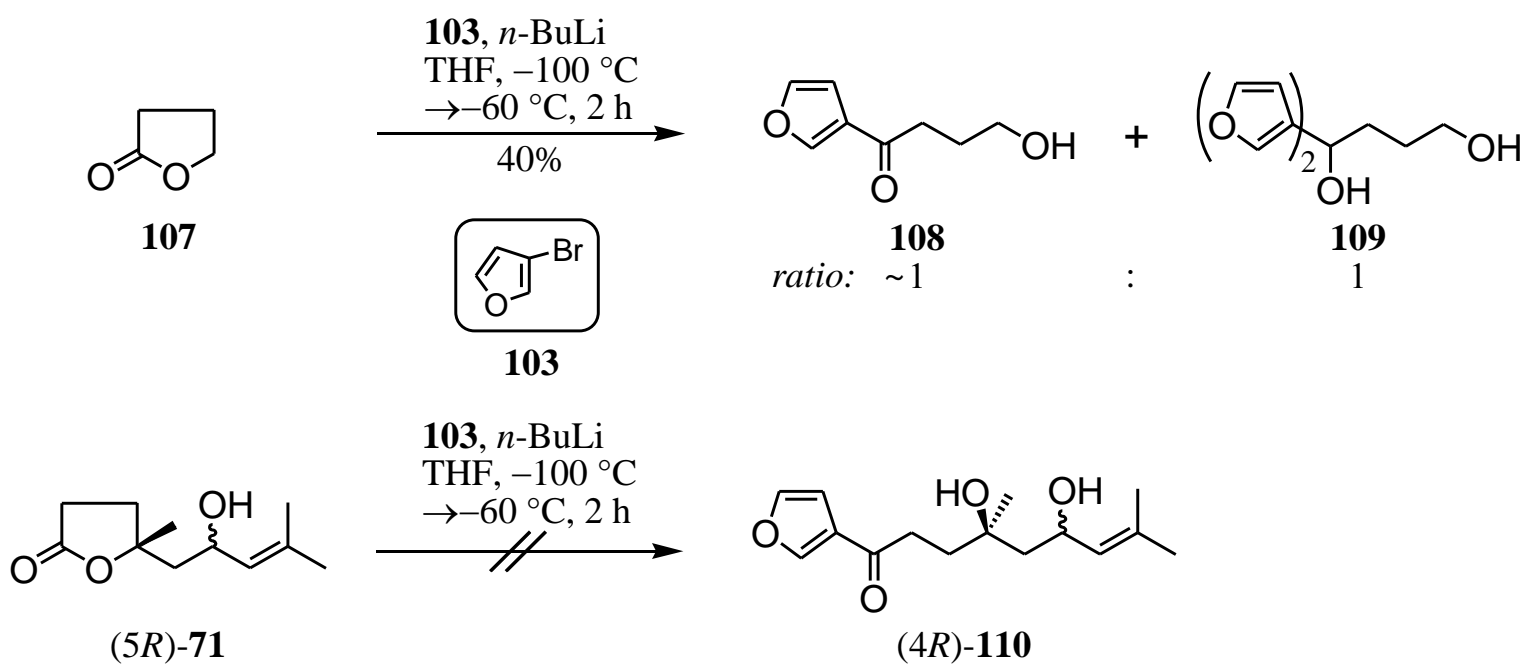

Schema 6-12: Addition von in situ generiertem 3-Furyllithium an GBL (107) und den Allylalkohol (5R)-71 unter den optimierten Reaktionsbedingungen.

Da die Reaktion mit (5R)-71 keinen Umsatz und mit GBL (107) lediglich schlechte Ausbeuten lieferte, wurde entschieden, die Furylgruppe nicht über die Addition an ein $\gamma$-Lacton, sondern über ein $\varepsilon$-Lacton, wie 102 einzuführen. Der $\varepsilon$-Lactonring kann nur gebildet werden, wenn die sich in $\gamma$-Position befindliche, tertiäre Hydroxylgruppe nicht für einen Ringschluss zur Verfügung steht. Folglich konnte die Auxiliargruppe erst nach Einführung der Furyleinheit abgespalten werden.

Zur Klärung, welche Abspaltungsmethode sich in Gegenwart eines acylierten Furans eignet, wurde das während der Optimierung angefallene 3-Acylfuran rac-104 jeweils einmal unter hydrogenolytischen und unter Birch-Bedingungen umgesetzt.

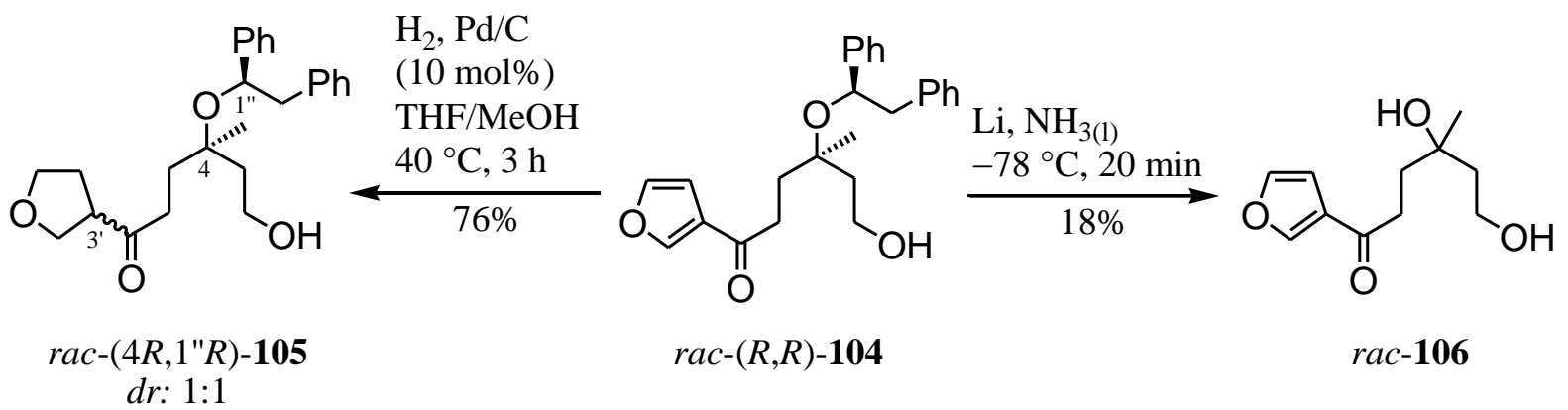

Schema 6-13: Verhalten des 3-Acylfurans rac-104 unter den reduktiven Abspaltungsbedingungen der Auxiliargruppe (links: Hydrogenolyse, rechts:Birch-Bedingungen).

Unter einer Wasserstoffatmosphäre und in Gegenwart von katalytischen Mengen Palladium auf Aktivkohle wurde noch vor der Abspaltung der Auxiliargruppe der Furanring der Verbindung 104 in 76\% Ausbeute unter Bildung des Tetrahydrofurans 105 als ein 1:1-Diastereomerengemisch hydriert. Aufgrund dessen wurde dieser 
Syntheseansatz nicht weiter verfolgt. Bei der Entschützung von 104 mit elementarem Lithium in flüssigem Ammoniak bei $-78{ }^{\circ} \mathrm{C}$ konnte das entschützte Produkt 106 in einer Ausbeute von $18 \%$ erhalten werden. Da davon ausgegangen wurde, dass die Abspaltung der Auxiliargruppe unter Birch-Bedingungen noch optimierbar sei, wurde der Ansatz zur Synthese des Furopinnatins (24) unter Verwendung eines $\varepsilon$-Lactons im Folgenden durchgeführt.

Als Ausgangsverbindungen dienten die beiden diastereomeren $\varepsilon$-Lactone (7R)-111 und (7S)-111, welche durch Addition des Vinylgrignards 101 an den Aldehyd 95 in THF bei $-60^{\circ} \mathrm{C}$ in einer Ausbeute von $67 \%$ im Verhältnis von 1:1.3 erhalten wurden. Da die in situ gebildete $\varepsilon$-Lactonfunktionalität von (7R)-111 und (7S)-111 gegenüber dem Grignard-Reagenz 101 eine vergleichbare Reaktivität wie die Aldehydgruppe in 95 aufwies, durfte kein größerer Überschuss an 101 (max. 1.2 Äq.) zugegeben werden, da es ansonsten verstärkt zu unerwünschten Additionsreaktionen von 101 unter Öffnung des $\varepsilon$-Lactonrings kam. Dies hatte allerdings zur Folge, dass ein vollständige Umsetzung von 95 im Laufe der Reaktion nicht erzielt werden konnte und nicht umgesetzter Aldehyd 95 im Anschluss per Säulenchromatographie wieder zurückgewonnen werden musste (s Schema 6-14).
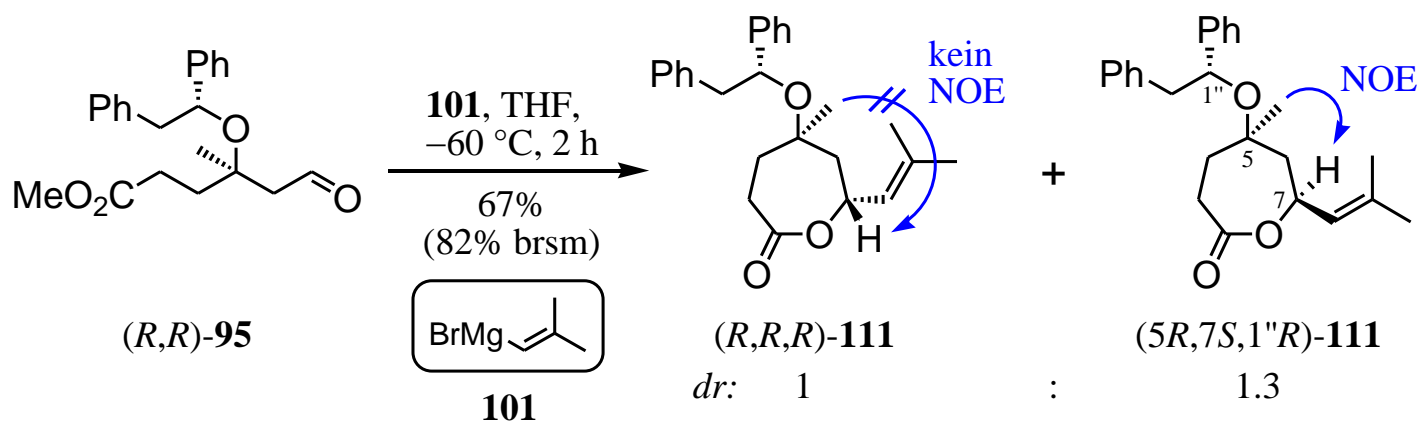

Schema 6-14: Addition des Vinylgrignards 101 an der Aldehyd $(R, R)-\mathbf{9 5}$.

Die Bestimmung der relativen Konfiguration beider Diastereomere konnte anhand einer NOE-Korrelation zwischen den Wasserstoffatomen der Methylgruppe an C-5 und dem 7-H ermittelt werden.

Die folgende Addition 3-Furyllithium an (7R)-111 bzw.(7S)-111 lieferte unter den optimierten Reaktionsbedingungen die beiden 3-Acylfurane (6R)-112 und (6S)-112 in akzeptablen Ausbeuten von 42\% bzw. 44\% (vgl. Modellsystem rac-102: 46\%). 
<smiles>CC(C)=C[C@@H]1C[C@]2(CCC(=O)O1)CCC(=O)O[C@H](c1ccccc1)C2</smiles>

$(7 R)-111$

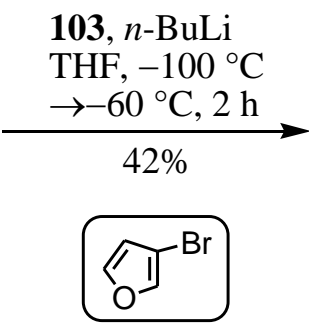

103<smiles>CC(C)=CC1C[C@](Cc2ccccc2)(OC(c2ccccc2)c2ccccc2)CCC(=O)O1</smiles>

(7S)-111<smiles>CC(C)=CC(O)C[C@](CCC(=O)c1ccoc1)(Cc1ccccc1)OC(c1ccccc1)c1ccccc1</smiles>

$(6 R)-112$<smiles></smiles>

$(6 S)-112$

Schema 6-15: Öffnung der $\varepsilon$-Lactone (7R)-111 und (7S)-111 mit in situ gebildetem 3-Furyllithium unter Bildung der 3-Acylfurane (6R)-112 und $(6 S)-\mathbf{1 1 2}$.

Zur Vervollständigung der Synthese von $(R)$-Furopinnatin (24) musste nun noch die Auxiliargruppe unter Birch-Bedingungen entfernt und die allylische Hydroxylgruppe analog der Synthese von $(R)$-Pinnatolid (25) mittels DMP-Oxidation in die Enonfunktionalität überführt werden. Jedoch konnte bereits nach der Entschützung der Auxiliargruppe -wahrscheinlich aufgrund von Zersetzung- kein Produkt mehr nachgewiesen werden. Die Wiederholung dieser Reaktion war aus Substanz- und Zeitgründen nicht mehr möglich.<smiles>CC(C)=C[C@H](O)C[C@](C)(CCC(=O)c1ccoc1)OC(Cc1ccccc1)c1ccccc1</smiles>

$(4 R, 1 " R)-112$ i) $\mathrm{Li}, \mathrm{NH}_{3(1)}$ $-78^{\circ} \mathrm{C}, 20 \mathrm{~min}$

ii) DMP<smiles>CC(C)=CC(=O)CC(C)(O)CCC(=O)c1ccoc1</smiles>

$(R)-24$

Schema 6-16: Abspaltung der Auxiliargruppe von $(4 R, 1 " R)-112$ unter Birch-Bedingungen und finale DMP-Oxidation zu $(R)$-Furopinnatin (24). 


\subsubsection{Diskussion spektroskopischer Daten des Acylfurans 112}

\subsubsection{1 ${ }^{1}$ H-NMR-Spektroskopie}

Das ${ }^{1}$ H-NMR-Spektrum des Acylfurans (6S)-112 zeigt im aromatischen Bereich $(\delta>6.5 \mathrm{ppm})$ neben dem Multiplett für die Protonen der beiden Phenylgruppen ebenfalls die Signale für die drei Protonen des Furanrings. Während das Signal bei $\delta=6.63 \mathrm{ppm}$ der Resonanz von 4'-H entspricht, resonieren die Protonen $2^{\prime}-\mathrm{H}$ und 5'-H aufgrund der Entschirmung durch das benachbarte Sauerstoffatom bei tieferem Feld. Dabei ist die vergleichsweise schwache ortho-Kopplung $\left({ }^{3} J=1.7 \mathrm{~Hz}\right)$ zwischen den Wasserstoffatomen 4'-H und 5'-H charakteristisch für den Furanring.

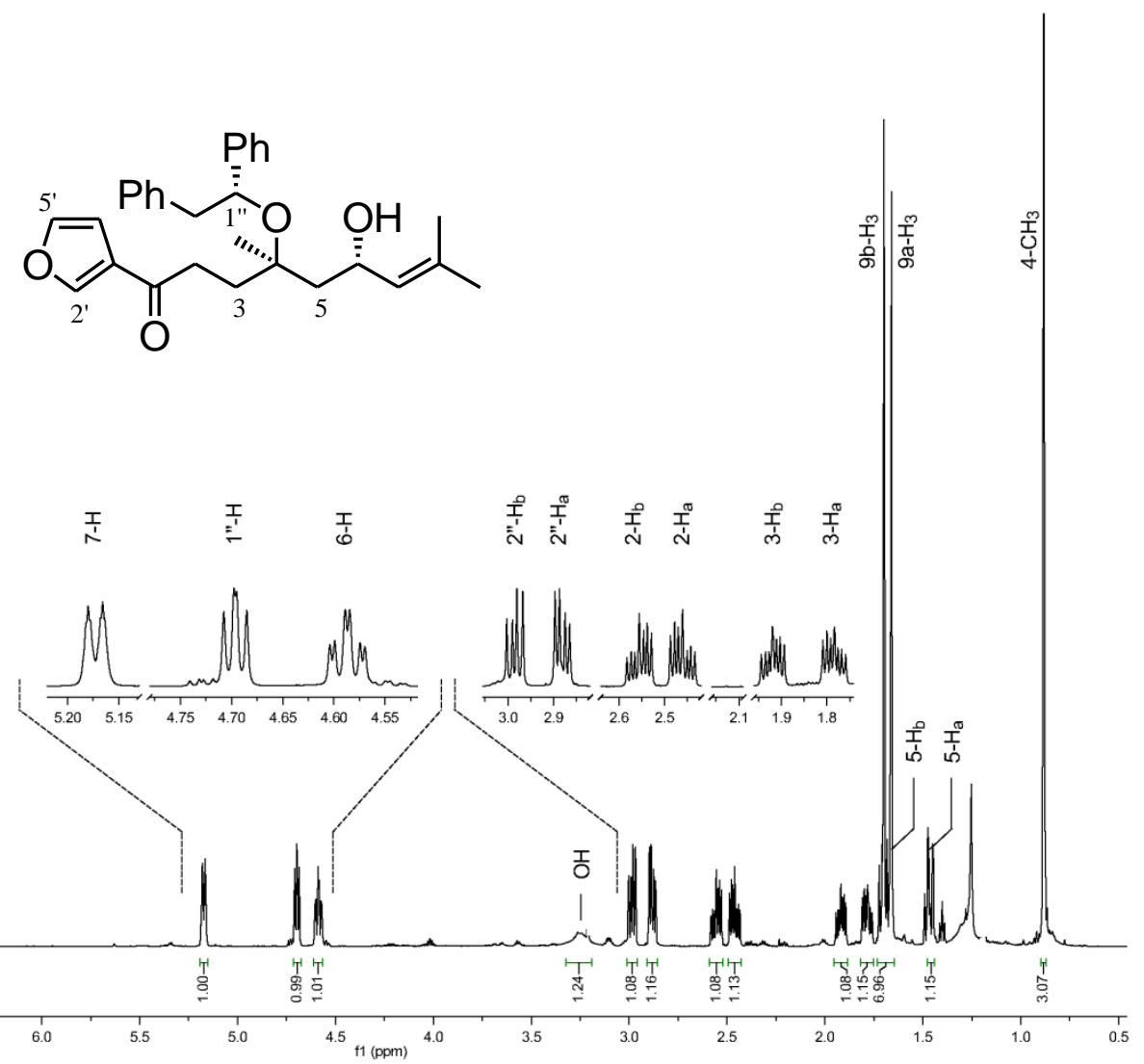

Abb. 6-12: ${ }^{1} \mathrm{H}-\mathrm{NMR}-$ Spektrum des Acylfurans (6S)-112.

Im Verschiebungsbereich von $\delta=4.5-5.3 \mathrm{ppm}$ resonieren zum einen das Wasserstoffatom an der Doppelbindung (7-H) und zum anderen das Proton 1"-H der Auxiliargruppe sowie 6-H, welches sich in Nachbarschaft zur Hydroxylgruppe befindet. Verantwortlich für die vergleichsweise starke Tieffeldverschiebung der Signale der aliphatischen Protonen 1"-H und 6-H ist neben einem jeweils benachbarten Sauerstoffatom die benzylische bzw. allylische Position in der sich die beiden 
Protonen befinden. Die diastereomeren Wasserstoffe der verbleibenden benzylischen Methylengruppe C-2" resonieren bei $\delta=2.88 \mathrm{ppm}$ und $\delta=2.99 \mathrm{ppm}$. Aus der geminalen Kopplung $\left({ }^{2} J=13.4 \mathrm{~Hz}\right)$ untereinander und je einer vicinalen Kopplung $\left({ }^{3} J=7.6,5.7 \mathrm{~Hz}\right)$ zum Proton 1"-H resultiert für diese Signale das Kopplungsmuster eines Dubletts vom Dublett. Während die diastereotopen Protonen der Methylengruppe an C-3 bei $\delta=1.78 \mathrm{ppm}$ und $\delta=1.92 \mathrm{ppm}$ resonieren, sind die Signale der diastereotopen Protonen am Methylenkohlenstoff C-2 mit $\delta=2.46 \mathrm{ppm}$ und $\delta=2.56 \mathrm{ppm}$ aufgrund der Entschirmung durch die benachbarte Ketogruppe weiter ins Tieffeld verschoben. Das Kopplungsmuster dieser Signale ergibt sich jeweils aus einer geminalen Kopplung $\left(3-\mathrm{H}_{2}:{ }^{2} J=14.3 \mathrm{~Hz}, 2-\mathrm{H}_{2}:{ }^{2} J=16.5 \mathrm{~Hz}\right)$ und zwei vicinalen Kopplungen $\left({ }^{3} J=10.1,5.4 \mathrm{~Hz}\right)$ zur benachbarten Methylengruppe. Die beiden Dubletts von Dubletts der diastereotopen Protonen der Methylengruppe an C-5 bei $\delta=1.46 \mathrm{ppm}$ und $\delta=1.70 \mathrm{ppm}$ sind teilweise mit den Resonanzen der allylischen Methylgruppen $9 \mathrm{a}-\mathrm{H}_{3}$ und $9 \mathrm{~b}-\mathrm{H}_{3}$ bei $\delta=1.66 \mathrm{ppm}$ und $\delta=1.70 \mathrm{ppm}$ überlagert. Analog zum Pinnatolid (25) resonieren die Protonen der beiden Methylgruppen aufgrund einer Fernkopplung $\left({ }^{4} \mathrm{~J}=1.0 \mathrm{~Hz}\right)$ zum Wasserstoffatom $7-\mathrm{H}$ jeweils als Dublett. Das verbleibende Singulett bei $\delta=0.88 \mathrm{ppm}$ entspricht schließlich der Resonanz der Protonen der aliphatischen Methylgruppe am C-4 (s. Abb. 6-12).

\subsubsection{2 ${ }^{13}$ C-NMR-Spektroskopie}

Das ${ }^{1} \mathrm{H}$-entkoppelte ${ }^{13} \mathrm{C}$-NMR-Spektrum des Acylfurans (6S)-112 zeigt insgesamt 10 Signale im aliphatischen Bereich $(\delta<100$ ppm), 14 Signale im Verschiebungsbereich von aromatischen und ungesättigten Kohlenstoffatomen $(\delta=100-160 \mathrm{ppm})$ sowie ein einzelnes, weit ins Tieffeld verschobenes Signal bei $\delta=194.3 \mathrm{ppm}$. Letzteres befindet sich im charakteristischen Verschiebungsbereich von Carbonylkohlenstoffen und entspricht somit dem Kohlenstoffatom C-1 der Ketogruppe. Die Intensitätsunterschiede bei den Signalen der aromatischen Kohlenstoffatome entstehen zum einen aus Symmetriegründen und zum anderen durch Unterschiede bei den Relaxationszeiten. Aufgrund der Symmetrie der Phenylgruppe (Punktgruppe: $\mathrm{C}_{2 \mathrm{v}}$ ) sind die ortho- bzw. meta-Kohlenstoffatome der jeweiligen Phenylgruppe chemisch identisch und ergeben folglich ein doppelt so intensives Signal wie beispielweise die para-Kohlenstoffatome. 


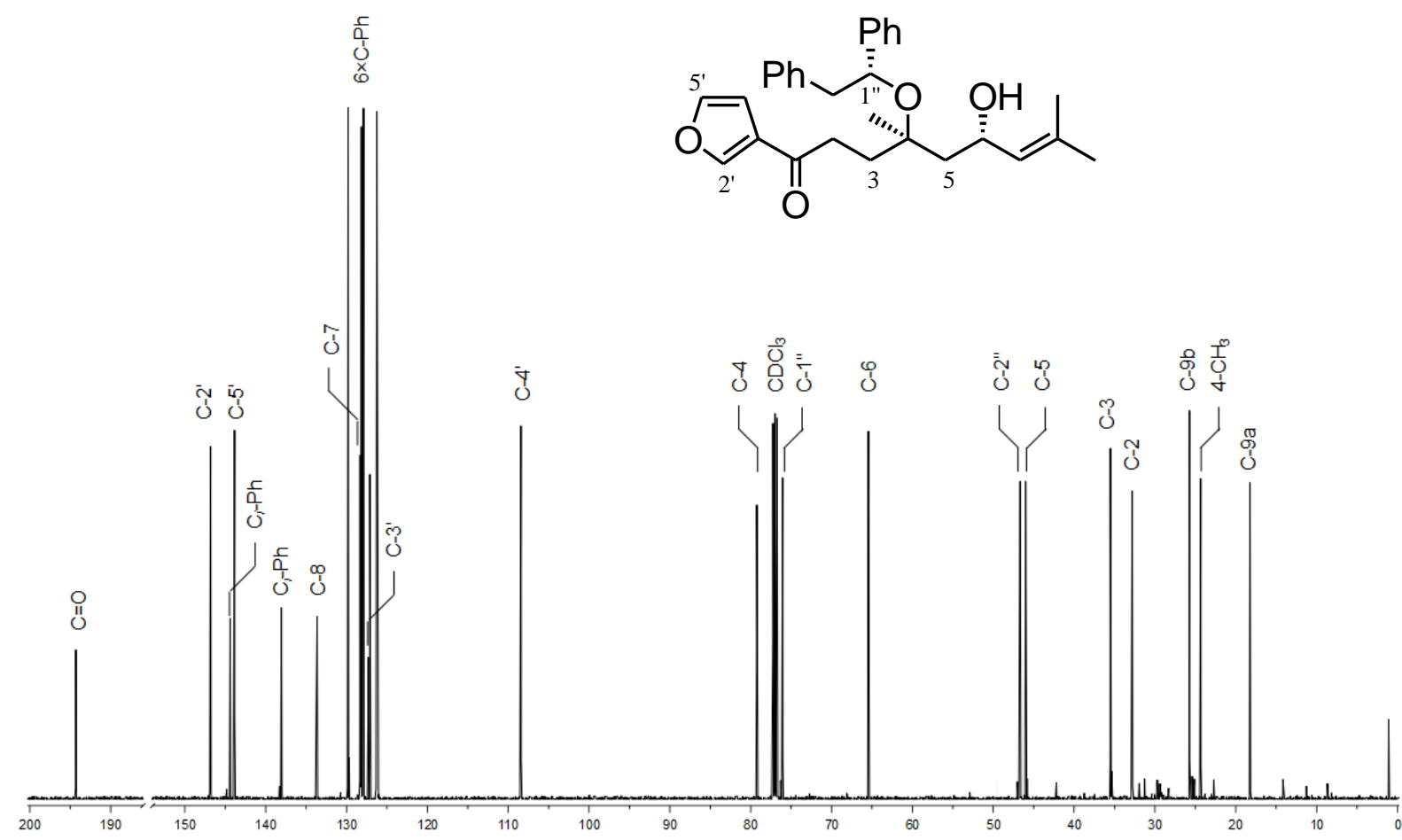

Abb. 6-13: ${ }^{13}$ C-NMR-Spektrum des Acylfurans (6S)-112.

Anhand der Tatsache, dass aus der langsameren Relaxation der quartären Kohlenstoffkerne eine geringere Signalintensität resultiert, lassen sich die Resonanzen der ipso-Kohlenstoffe der Phenylgruppen, dem Doppelbindungskohlenstoffatom C-8 sowie dem Furankohlenstoff C-3' zuordnen. Die Signale von C-3' und dem anderen Doppelbingungskohlenstoff C-7 fallen dabei mit den Verschiebungsbereich der Phenylkohlenstoffatome zusammen. Während die Furankohlenstoffe C-2' und C-5' aufgrund der Entschirmung durch das benachbarte Furansauerstoffatom bei einer chemischen Verschiebung von $\delta=146.8 \mathrm{ppm}$ und $\delta=143.9 \mathrm{ppm}$ resonieren, ist das Signal für C-4' bei $\delta=108.4 \mathrm{ppm}$ deutlich zu höherem Feld verschoben. Im aliphatischen Bereich des Spektrums fallen zunächst die Signale für die drei Stereozentren C-4, den C-1" und C-6 auf, welche jeweils aufgrund der Entschirmung durch ein benachbartes Sauersfoffatom $(\delta>50 \mathrm{ppm})$ bei tieferem Feld resonieren als die restlichen aliphatischen Kohlenstoffatome. Die verbleibenden Signale entsprechen schließlich der benzylischen Methylengruppe C-2", den Methylgruppen C-5, C-3 und $\mathrm{C}-2$ sowie den drei Methylgruppen 4- $\mathrm{CH}_{3}, \mathrm{C}-9 \mathrm{a}$ und $\mathrm{C}-9 \mathrm{~b}$. 


\section{$7 \quad$ Zusammenfassung}

Asymmetrische Allylierungen von Carbonylverbindungen zählen im Bereich der enantioselektiven Totalsynthese von Naturstoffen und anderer biologisch aktiver Substanzen zu den wichtigsten C-C-Verknüpfungsreaktionen. Wahrend für die enantioselektive Darstellung von sekundären Homoallylalkoholen eine Vielzahl von hochselektiven Verfahren existieren, stehen für die enantioselektive Synthese von tertiären Homoallylalkoholen nur wenige asymmetrische Allylierungsmethoden mit guten bis sehr guten Selektivitäten zur Verfügung. Eine herausragende Methode auf dem Gebiet der Allylierung von prochiralen aliphatischen Ketonen ist die Multikomponenten-Allylierungsreaktion (MCAR) nach L. F. Tietze mit dem PhenylBenzyl-Auxiliar (60).

Für erste totalsynthetische Anwendungen der MCAR mit dem Phenyl-BenzylAuxiliar (60) in der Naturstoffsynthese wurden die beiden isoprenoiden Naturstoffe Pinnatolid (25) und Furopinnatin (24) ausgewählt.<smiles>CC(C)=CC(=O)C[C@](C)(O)CCC(=O)c1ccoc1</smiles>

$(R)-24$

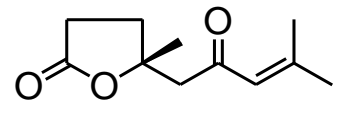

$(R)-25$

Abb. 7-1: $(R)$-Furopinnatin (24) und (R)-Pinnatolid (25).

Die Retrosynthese von 24 und 25 führt über den Allylalkohol $\mathbf{7 1}$ via Weg A zum Keton Sulcaton (76) und via Weg B zum $\gamma$-Ketoester Lävulinsäuremethylester (86a).

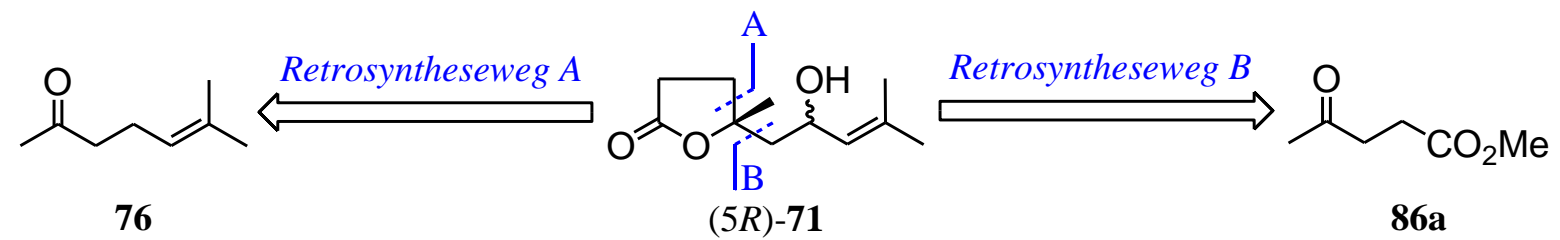

Schema 7-1: Retrosynthetische Schnitte für die Allylierungsvarianten A und B. 


\subsection{Synthese des $(R)$-Phenyl-Benyl-Auxiliars}

Zur enantioselektiven Synthese des chiralen Auxiliars (R)-60 wurde käuflich erhältliches Desoxybenzoin (72) nach einem modifizierten Protokoll der Corey-ItsunoReduktion $^{[37,38]}$ mit Diphenyl-L-prolinol (73) ${ }^{[39]}$ als Präkatalysator in quantitativer Ausbeute und einem Enantiomerenüberschuss von $84 \%$ ee in die Auxiliarvorstufe $(R)$-74 überführt. Die nahezu vollständige Enantiomerenanreicherung von $(R)$-74 gelang durch Umkristallisation des entsprechenden Dinitrobenzoesäureester $(R)-75$ mit einer Gesamtausbeute von 68\% über zwei Stufen. Nach Silylierung von $(R)-74$ mit TMSOTf wurde das Phenyl-Benzyl-Auxiliar $(R)-\mathbf{6 0}$ in 93\% Ausbeute erhalten.

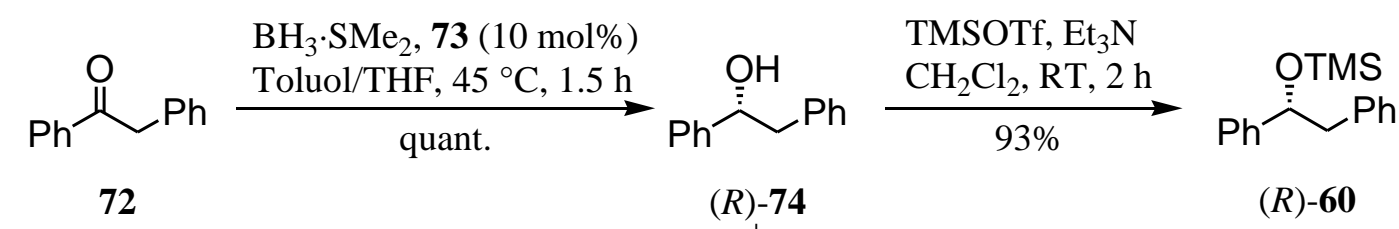

$84 \%$ ee $\mid>99 \%$ ee

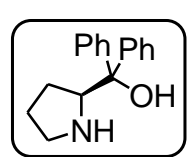

73

$$
\begin{aligned}
& \mathrm{DNBCl,Et}+\mathrm{N}, \\
& \begin{array}{l}
\mathrm{DMAP}(10 \mathrm{~mol} \%) \\
\mathrm{CH}_{2} \mathrm{Cl}_{2}, \mathrm{RT}, 3 \mathrm{~h}
\end{array}
\end{aligned} \mid \begin{aligned}
& \mathrm{LiOH \cdot \textrm {H } _ { 2 } \mathrm { O } ,} \\
& \mathrm{CH}_{2} \mathrm{Cl}_{2} / \mathrm{MeOH} / \\
& \mathrm{H}_{2} \mathrm{O}, \mathrm{RT}, 1 \mathrm{~h} \\
& \text { quant. }
\end{aligned}
$$

Schema 7-2: $\quad$ Herstellung des Phenyl-Benzyl-Auxiliars $(R)-60$. 


\subsection{Syntheseweg A}

Die Allylierung von Sulcaton (76) unter Verwendung des Phenyl-Benzyl-Auxiliars $(R)$-60 führte in einer sehr guten Ausbeute von 91\% und einer guten Diastereoselektivität von ca. 90:10 zum Homoallylether $(R, R)-77$. Nachfolgend wurde die terminale Doppelbindung in $\mathbf{7 7}$ mit 9-BBN regioselektiv in 86\% Ausbeute hydroboriert. Der gebildete primäre Alkohol 78 wurde mit DMP- und anschließender Pinnick-Oxidation in 60\% Ausbuete weiter zur Säure $(R, R)-80$ umgesetzt. Nach Abspaltung der Auxiliargruppe unter Birch-Bedingungen konnte das $\gamma$-Lacton $(R)$-82 in 79\% Ausbeute gebildet werden. Die finale allylische Oxidation zum $(S)$-Pinnatolid (25) konnte jedoch lediglich in mäßigen Ausbeuten von ca. 30\% erreicht werden (s. Schema 7-3).
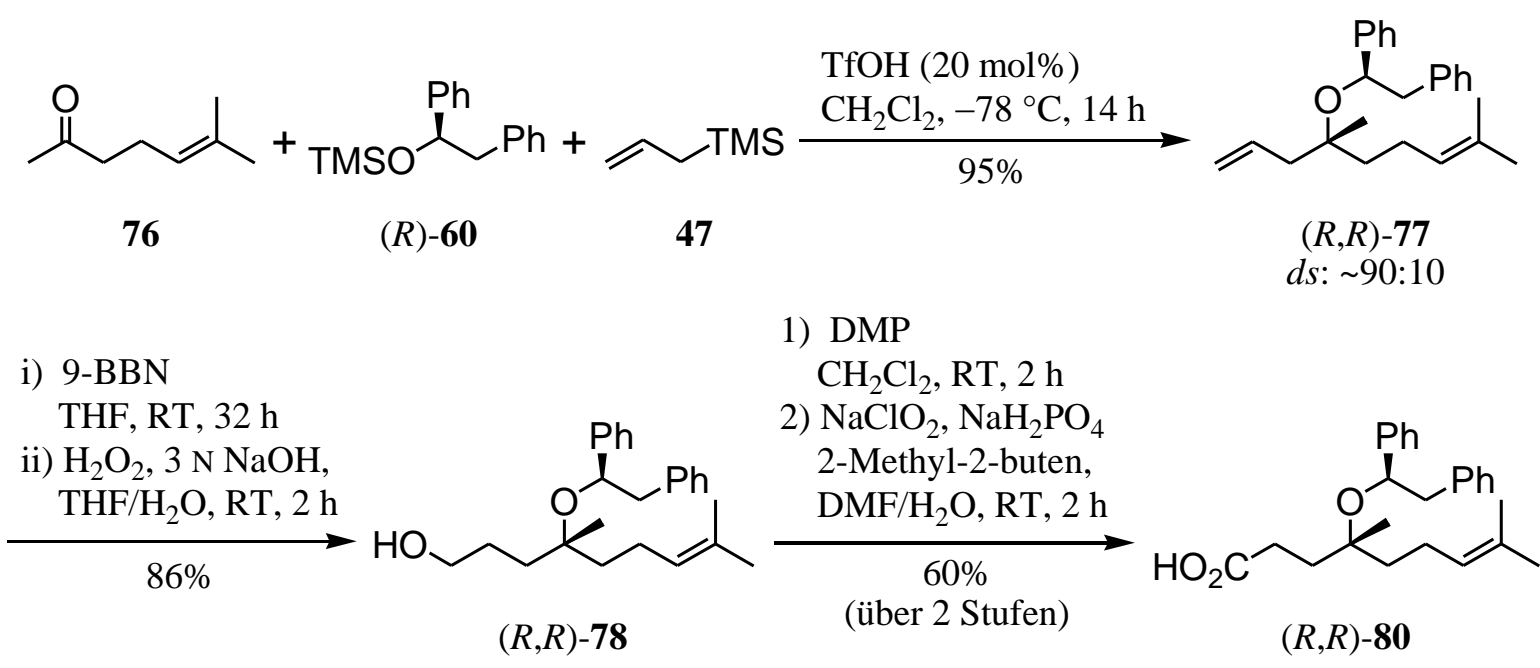

1) DMP

$\mathrm{CH}_{2} \mathrm{Cl}_{2}, \mathrm{RT}, 2 \mathrm{~h}$

2) $\mathrm{NaClO}_{2}, \mathrm{NaH}_{2} \mathrm{PO}_{4}$ 2-Methyl-2-buten, $\mathrm{DMF} / \mathrm{H}_{2} \mathrm{O}, \mathrm{RT}, 2 \mathrm{~h}$

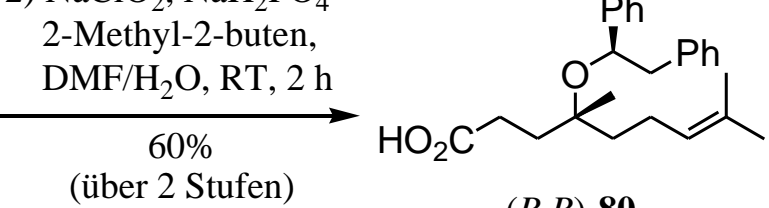

i) $\mathrm{Li}, \mathrm{NH}_{3(1)}$, $-78{ }^{\circ} \mathrm{C}, 20 \mathrm{~min} \quad \mathrm{Mn}(\mathrm{OAc})_{3}(20 \mathrm{~mol} \%)$

ii) $\mathrm{MeI}, \mathrm{K}_{2} \mathrm{CO}_{3}$, TBHP, MS 4 A

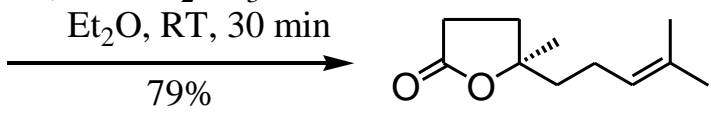

$(R)-82$

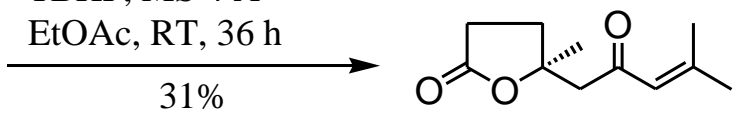

$(S)-25$

$72 \% e e$

Schema 7-3: Syntheseweg A.

Aufgrund des lediglich moderaten Enantiomerenüberschusses von $72 \%$ ee und den mäßigen Ausbeuten bei der allylischen Oxidation, wurde im Folgenden die Synthese von Furopinnatin (24) nach Synthesesweg B untersucht. 


\subsection{Syntheseweg B}

Die Umsetzung von Lävulinsäuremethylester (86a) mit dem Allylsilan 47 in Gegenwart von $(R)-\mathbf{6 0}$ führte mit einer sehr guten Ausbeute von $91 \%$ und einer exzellenten Diastereoselektivität von 94:6 zum Homoallylether $(R, R)$-89a. Es kann angenommen werden, dass die hohe Selektivität bei der Allylierung der $\gamma$-Ketoesters 86a-c (s. Tabelle 7-1) auf einen Nachbargruppeneffekt der Esterfunktion zurückzuführen ist. Selbst die Umsetzung des $\delta$-Ketoesters 87 lieferte noch eine gute Diastereoselektivität von 91:9.

Tabelle 7-1: Asymmetrische Allylierung von Ketoestern nach L. F.Tietze.

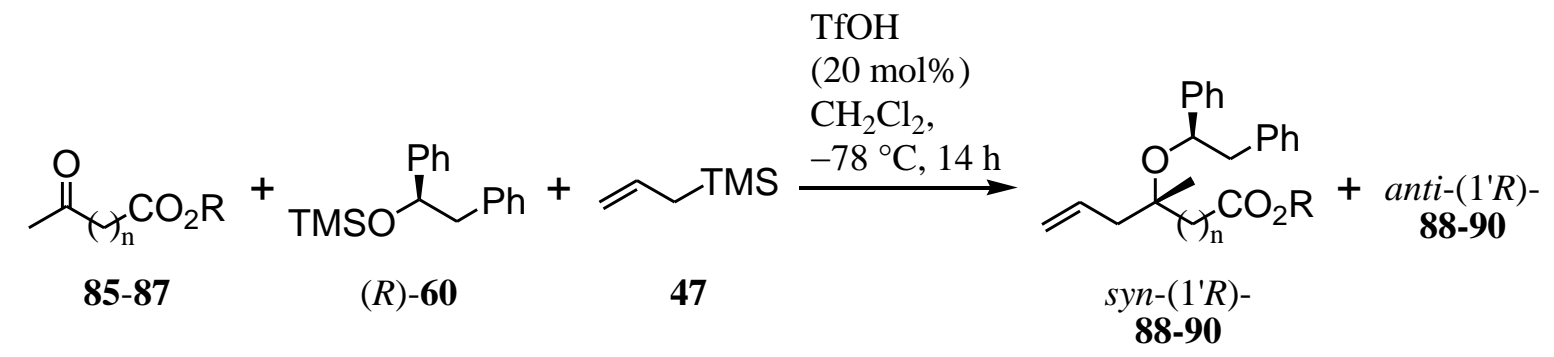

\begin{tabular}{c|c|l|c|c|c}
\hline No. & \multicolumn{2}{|l|}{ Ketoester } & Homoallylether & Ausbeute [\%] & syn/anti \\
\hline $\mathbf{1}$ & $\mathbf{8 5}$ & $\mathrm{n}=1, \mathrm{R}=\mathrm{Me}$ & $\mathbf{8 8}$ & - & - \\
\hline $\mathbf{2}$ & $\mathbf{8 6 a}$ & $\mathrm{n}=2, \mathrm{R}=\mathrm{Me}$ & $\mathbf{8 9 a}$ & 91 & $94: 6$ \\
$\mathbf{3}$ & $\mathbf{8 6 b}$ & $\mathrm{n}=2, \mathrm{R}=i-\mathrm{Pr}$ & $\mathbf{8 9 b}$ & 94 & $94: 6$ \\
$\mathbf{4}$ & $\mathbf{8 6 c}$ & $\mathrm{n}=2, \mathrm{R}=t-\mathrm{Bu}$ & $\mathbf{8 9 c}$ & $66^{[\mathrm{a}]}$ & $93: 7$ \\
\hdashline $\mathbf{5}$ & $\mathbf{8 7}$ & $\mathrm{n}=3, \mathrm{R}=\mathrm{Me}$ & $\mathbf{9 0}$ & 98 & $91: 9$ \\
\hline
\end{tabular}

[a] Auxiliar rac-60 wurde verwendet.

Nach oxidativer Spaltung der Doppelbindung in 89a durch Ozonolyse und reduktiver Aufarbeitung wurde der primäre Alkohol 96 in nahezu quantitativer Ausbeute erhalten. Die Reinigung von $(R, R)-96$ erfolgte durch Umkristallisation des entsprechenden 3,5-Dinitrobenzoesäureesters 97. Anhand einer Kristallstruktur von $(R, R)-97$ konnte ebenfalls die vom Mechanismus postulierte absolute Konfiguration zweifelsfrei belegt werden. Die hydrogenolytische Abspaltung der Auxiliargruppe in 96 führte in quantitativer Ausbeute direkt zum $\gamma$-Lacton $(R)$-98. DMP-Oxidation von 98 zum Aldehyd 99, Umsetzung mit dem Vinylgrignard 101 zum Allylalkohol 71 und 
nachfolgende erneute Oxidation mit DMP lieferten das $R$-Enantiomer des Naturstoffs Pinnatolid (25) in 36\% Ausbeute. Die Enantiomerenreinheit wurde mittels HPLC an chiraler Phase mit $>99 \%$ ee bestätigt werden. Zur Synthese von Furopinnatin (24) wurde das $\gamma$-Lacton $\mathbf{7 1}$ mit in situ gebildetem 3-Furyllithium umgesetzt. Das gewünschte Additionsprodukt 110 wurde dabei jedoch nicht erhalten (s. Schema 7-4).

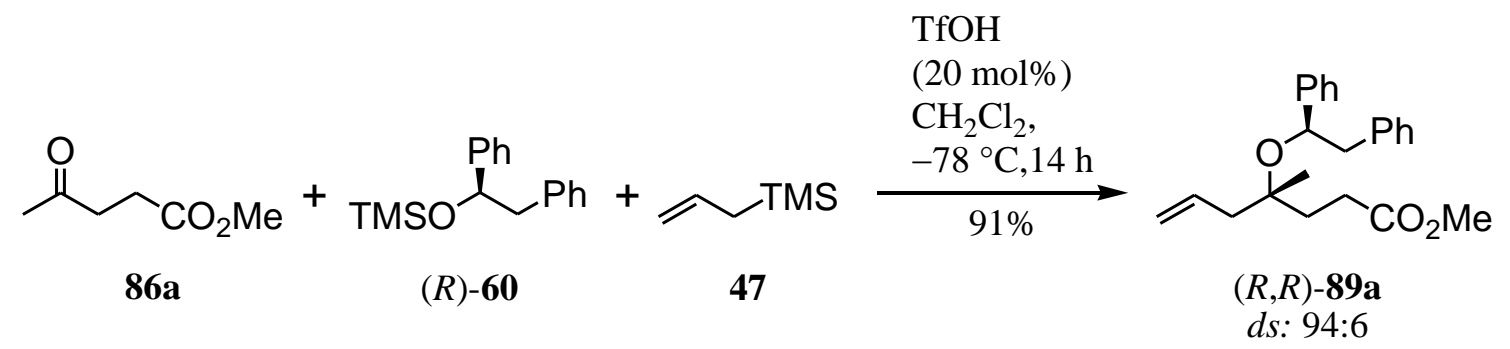

i) $\mathrm{O}_{3}, \mathrm{PPh}_{3}$ $\mathrm{CH}_{2} \mathrm{Cl}_{2} / \mathrm{MeOH}$, $-78^{\circ} \mathrm{C} \rightarrow \mathrm{RT}, 14 \mathrm{~h}$

ii) $\mathrm{NaBH}_{4}$ $\mathrm{MeOH}, \mathrm{RT}, 0.5 \mathrm{~h}$ $98 \%$
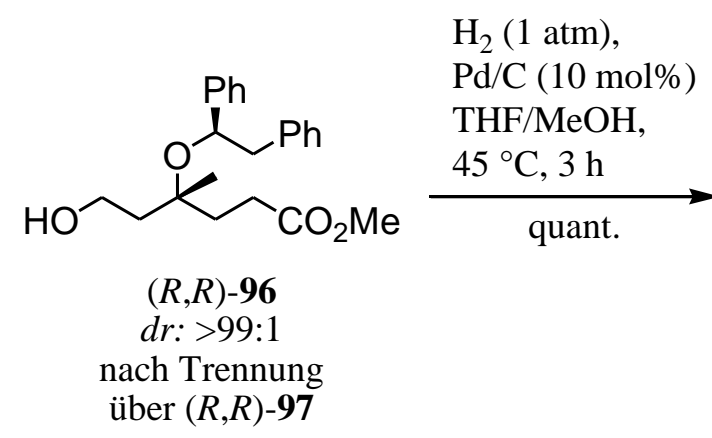

$(R)-98$

i) $\mathrm{DMP}$

$\mathrm{CH}_{2} \mathrm{Cl}_{2}, \mathrm{RT}, 30 \mathrm{~min}$

ii) 101 ,

$\underset{40 \%}{\mathrm{THF},-60{ }^{\circ} \mathrm{C}, 2 \mathrm{~h}}$ (über 2 Stufen)

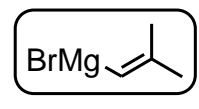

101

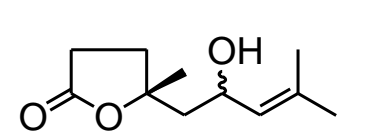

$(5 R)-71$

$d s: 1: 1.1$

\section{DMP}

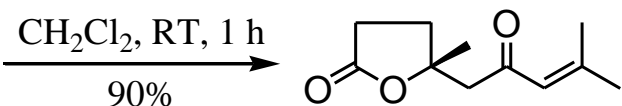

(R)-25

$>99 \%$ ee

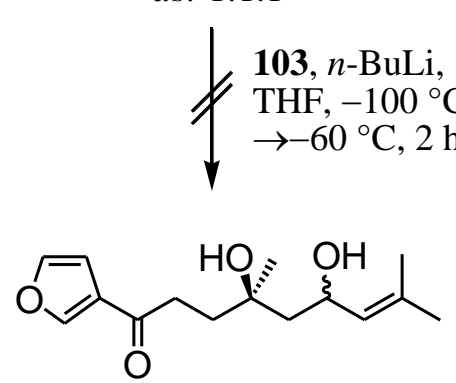

(4R)-110

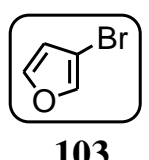

103

Schema 7-4: $\quad$ Syntheseweg B.<smiles>CC(C)=CC(=O)C[C@@H](O)CCC(=O)c1ccoc1</smiles>

(R)-24 
Alternativ konnte der Aldehyd 95 mit dem Grignard-Reagenz 101 in 67\% Ausbeute zu dem $\varepsilon$-Lacton 111 transformiert werden. Addition von 3-Furyllithium führte in akzeptablen Ausbeuten zum Furanderivat 112. Leider gelang es jedoch nicht, die Schutzgruppe in $\mathbf{1 1 2}$ ohne Zersetzung des Moleküls abzuspalten.

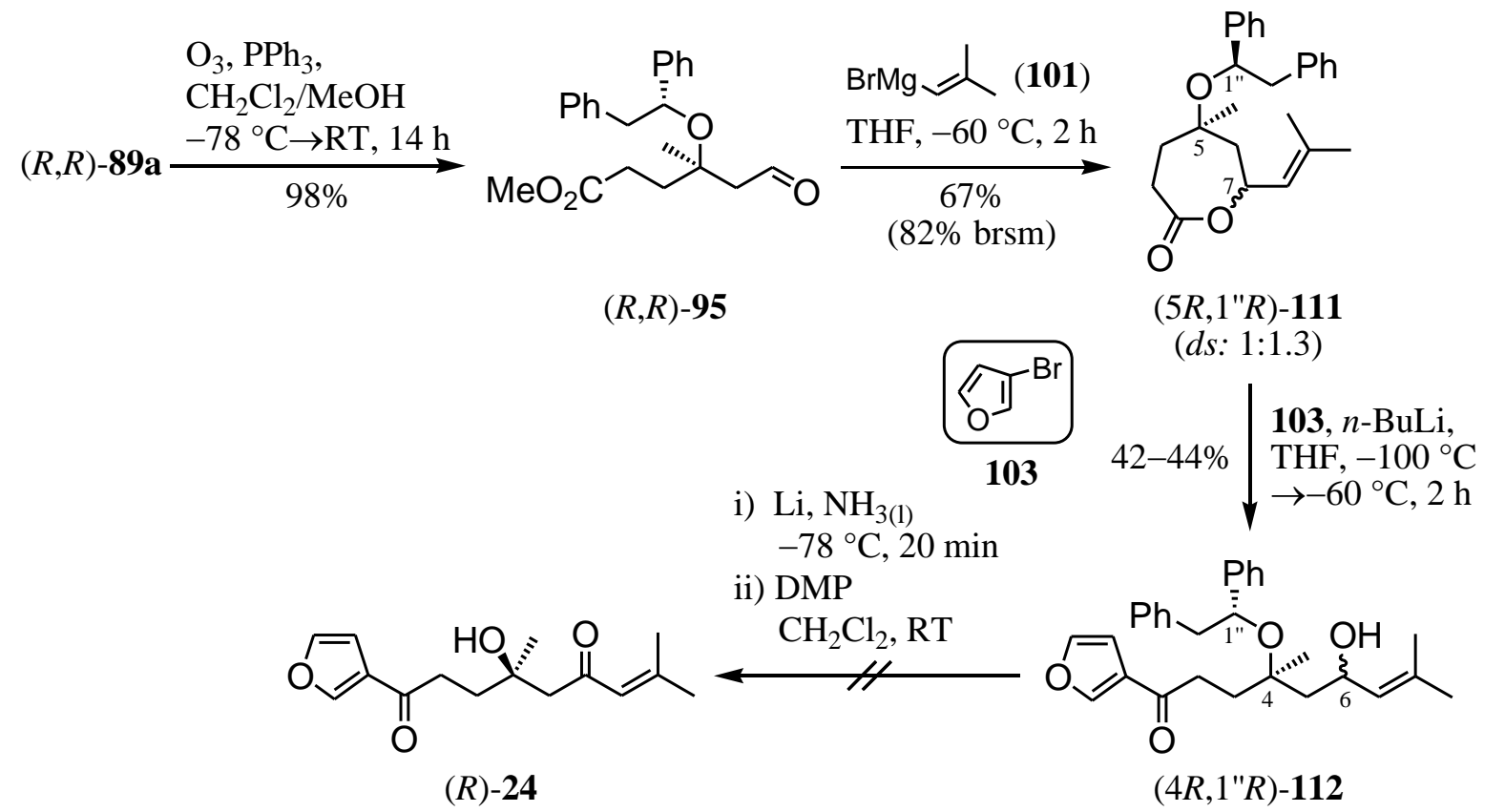

Schema 7-5: Alternativvariante des Syntheseweges B. 


\section{B. EXPERIMENTELLER TEIL}

\section{Allgemeine Arbeitsvorschriften}

Die Umsetzungen wurden, soweit nötig, in ausgeheizten Glasapparaturen unter einem leichten Argon-Überdruck durchgeführt. Die Lösungsmittel wurden entsprechend den üblichen Labormethoden ${ }^{[51]}$ getrocknet bzw. p.a.-Ware über Molekularsieb gelagert. Soweit nicht anders vermerkt, wurden kommerziell erhältliche Produkte ohne weitere Reinigung eingesetzt. Das Entgasen von Lösungsmitteln geschah durch Einleiten eines Argongasstroms über einen längeren Zeitraum. Konzentrationsangaben beziehen sich, sofern nicht anders vermerkt, auf wäßrige Lösungen.

\section{$1.1 \quad$ Verwendete Geräte}

Drehwerte: Drehwerte wurden mit einem Polarimeter Modell 241 der Firma PerkinElmer sowie dem Modell P-2000 der Firma Jasco gemessen.

CD-Spektren: Das CD-Spektrum wurde mit dem Spektropolarimeter Modell J-810 der Firma Jasco aufgenommen.

Infrarotspektren: Infrarotspektren wurden zum einen mit dem Modell Vector 22 der Firma Bruker, wobei Feststoffe als KBr-Presslinge und Flüssigkeiten als Film zwischen KBr-Platten gemessen wurden, und zum anderen mit dem ATR-Modell FT/IR-4100 der Firma Jasco aufgenommen.

UV/VIS-Spektren: Die Aufnahme der UV/VIS-Spektren erfolgte mit den Modellen Lambda 2 der Firma Perkin-Elmer und V-630 der Firma Jasco.

${ }^{1}$ H-NMR-Spektren: Die ${ }^{1}$ H-NMR-Spektren wurden mit den Modellen Mercury 300, Mercury-vx 300, Unity 300, Vnmrs 300 (jeweils $300 \mathrm{MHz}$ ) sowie Inova 600 (600 MHz) der Firma Varian von in deuterierten Solventien gelösten Proben aufgenommen. Die chemischen Verschiebungen sind in Einheiten der $\delta$-Skala angegeben und auf das 
Signal des angegebenen Lösungsmittels referenziert. Zur Kennzeichnung der Multiplizitäten der Signale werden folgende Abkürzungen verwendet: s (Singulett), d (Dublett), t (Triplett), q (Quartett), dd (Dublett von Dublett), dt (Dublett von Triplett) usw. Signale, die durch Überlagerung oder Anteile höherer Ordnung nicht interpretierbar waren, wurden mit $\mathrm{m}$ (Multiplett) bzw. $\mathrm{m}_{\mathrm{c}}$ (symmetrisches, zentriertes Multiplett) bezeichnet und verbreiterte Signale durch den Zusatz „br.“ gekennzeichnet.

${ }^{13}$ C-NMR-Spektren: Die ${ }^{1} \mathrm{H}$-breitband entkoppelten ${ }^{13} \mathrm{C}$-NMR-Spektren wurden mit den Geräten Unity 300 (75 MHz), Inova 500 (125 MHz) und Inova 600 (150 MHz) der Firma Varian aufgenommen. Die chemischen Verschiebungen sind in Einheiten der $\delta$-Skala angegeben. Als interner Standard diente das angegebene Lösungsmittel. Für phenylische Kohlenstoffatome werden die folgende Abkürzungen verwendet: $i$ (ipso), $o$ (ortho), $m$ (meta), $p$ (para).

Massenspektren: Zur Aufnahme der EI- und EI-HRMS-Spektren diente das Time-ofFlight Massenspektrometer AccuTOF der Firma Jeol. ESI-Spektren wurden mit einem Ion-Trap-Massenspektrometer LCQ der Firma Finnigan sowie dem Time-of-Flight Massenspektrometer microTOF der Firma Bruker aufgenommen. Die Messung der ESI-HRMS-Spektren erfolgte an einem 7-Tesla-Fourier-Transform-Ion-CyclotronResonance (FTICR)-Massenspektrometer APEX IV der Firma Bruker und am microTOF-Gerät. Angegeben werden die Quotienten aus Masse zu Ladung sowie in Klammern die relativen Intensitäten bezogen auf den Basispeak $(I=100)$.

Kältetechnik: Die Reaktionsführung bei konstant tiefen Temperaturen über einen längeren Zeitraum erfolgte unter Einsatz von Kryostaten. Benutzt wurde das Modell EK 90 der Firma Haake. Darüber hinaus wurden übliche Kältemischungen wie Eis / Wasser, Aceton / Trockeneis und Ethanol / Stickstoff zur temporären Kühlung verwendet. 


\subsection{Chromatographische Methoden}

Dünnschichtchromatographie (DC): Es wurden Aluminium-Fertigfolien Si $60 \mathrm{~F}_{254}$ der Firma Merck verwendet. Angegeben sind $\mathrm{R}_{f}$-Werte (Laufhöhe relativ zur Laufmittelfront). Neben der UV-Detektion diente eine Vanillin-Schwefelsäure-Lösung (0.5 g Vanillin, $3 \mathrm{~mL}$ konz. $\mathrm{H}_{2} \mathrm{SO}_{4}, 85 \mathrm{~mL} \mathrm{MeOH}$ und $10 \mathrm{~mL} \mathrm{HOAc}$ ) als Reagenz zur Anfärbung.

Säulenchromatographie: Alle säulenchromatographischen Trennungen wurden mit Kieselgel 60 (Korngröße: 0.032-0.063 mm) der Firma Merck durchgeführt.

\section{Hochdruckflüssigkeitschromatographie (HPLC):}

Analytische Trennungen wurden auf einer HPLC-Anlage der Firma Jasco, ausgestattet mit einer Lösungsmittelpumpe PU-2080, der Mischkammer LG-1590-04, dem Multiwellenlängendetektor MD-2010 Plus und der Steuerung LC-Net II/ADC, vorgenommen. Für die Injektion wurde ein automatischer Probenwechsler (Autosampler AS-2055) derselben Firma verwendet. Zur Bedienung, Datenerfassung und Datenauswertung wurden die Computerprogramme Borwin PDA, HSS 2000 und Borwin Chromatography der Firma Jasco eingesetzt. Für die analytischen Messungen wurde Säulen mit den chiralen stationären Phasen Chiralpak ${ }^{\circledR}$ IA $(250 \times 4.6 \mathrm{~mm}$, $5 \mu \mathrm{m}$, Daicel Chemical Industries Ltd. $)$ und Chiralpak ${ }^{\circledR} \mathrm{IB}(250 \times 4.6 \mathrm{~mm}, 5 \mu \mathrm{m}$, Daicel Chemical Industries Ltd.) verwendet. Angegebene Lösungsmittel waren von HPLC-Qualität und alle Proben wurden zunächst über Polytetrafluorethylen-(PTFE)Filter der Firma Roth (Ø $25 \mathrm{~mm}, 0.2 \mu \mathrm{m})$ bzw. VWR (Ø $13 \mathrm{~mm}, 0.2 \mu \mathrm{m})$ membranfiltriert. 


\subsection{Allgemeine Reaktionsvorschriften}

\subsubsection{Tietze-Allylierung von prochiralen Methylketonen (AAV1)}

$\mathrm{Zu}$ einer Lösung eines prochiralen Methylketons (1.5 M), Phenyl-Benzyl-Auxiliar (60) $(1.5 \mathrm{M})$ und Allyltrimethylsilan (47) $(1.7 \mathrm{M})$ in $\mathrm{CH}_{2} \mathrm{Cl}_{2}$ wurde bei $-78^{\circ} \mathrm{C}$ langsam TfOH $(0.3 \mathrm{M}, 20 \mathrm{~mol} \%)$ gegeben und $14 \mathrm{~h}$ bei dieser Temperatur gerührt. Die Reaktion wurde durch Zugabe von $\mathrm{Et}_{3} \mathrm{~N}(0.1 \mathrm{~mL} / \mathrm{mmol}$ Keton) beendet und das Lösungsmittel unter reduziertem Druck entfernt. Säulenchromatographische Reinigung des Rückstands an Kieselgel (PE/MTBE) lieferte den Homoallylether.

\subsubsection{Abspaltung der Auxiliargruppe durch Hydrogenolyse (AAV2)}

Unter einer Argonatmosphäre wurde zu einer entgasten Lösung des 1,2-Diphenylethylethers (ca. $0.1 \mathrm{M}$ ) in THF/MeOH 2:1 bei Umgebungstemperatur $10 \mathrm{w} \%$ Palladium auf Aktivkohle (ca. $0.01 \mathrm{M}, 10 \mathrm{~mol} \%$ ) gegeben. Danach wurde die Lösung erneut entgast, die Atmosphäre zu Wasserstoff getauscht und anschließend die Suspension auf $45^{\circ} \mathrm{C}$ erwärmt. Nach vollständigem Umsatz des Substrates (DC-Kontrolle) wurde die Suspension über Kieselgur filtriert, mit $\mathrm{CH}_{2} \mathrm{Cl}_{2}(3 \times)$ gewaschen und das Lösungsmittel unter reduziertem Druck entfernt. Nach säulenchromatographischer Reinigung an Kieselgel wurden die entschützten Alkohole erhalten.

\subsubsection{Abspaltung der Auxiliargruppe unter Birch-Bedingungen (AAV3)}

$\mathrm{Zu}$ einer tiefblauen Lösung von Lithium (ca. $8 \mathrm{mg}$ ) in flüssigem Ammoniak (ca. $30 \mathrm{~mL}$ ) wurde bei $-78{ }^{\circ} \mathrm{C}$ tropfenweise der 1,2-Diphenylethylether (ca. $1.5 \mathrm{M} \mathrm{Lsg}$. in $\mathrm{Et}_{2} \mathrm{O}$ oder THF) gegeben und 20 min bei dieser Temperatur intensiv gerührt (Vorsicht: leicht stockende Lösung). Die Reaktion wurde durch Zugabe von festem $\mathrm{NH}_{4} \mathrm{Cl}$ (ca. $80 \mathrm{mg}$ ) beendet und anschließend wurde die Kühlung der Reaktionslösung zum Verdampfen des Ammoniaks entfernt (Dauer: ca. 2-4 h, Vorsicht: Siedeverzüge). Der Rückstand wurde in $\mathrm{CH}_{2} \mathrm{Cl}_{2}$ aufgenommen, die Suspension über Kieselgur filtriert, mit $\mathrm{CH}_{2} \mathrm{Cl}_{2}$ (3x) gewaschen und das Lösungsmittel unter reduziertem Druck entfernt. Nach säulenchromatographischer Reinigung an Kieselgel wurden die entschützten Alkohole erhalten. 


\section{Synthese des $(R)$-Phenyl-Benzyl-Auxiliars 60}

\section{$2.1 \quad(-)-(R)-1,2-D i p h e n y l e t h a n o l ~(74)$}<smiles>O[C@H](C[PH])c1ccccc1</smiles>

A: Zu einer Lösung von Diphenyl-L-prolinol (73) $(265 \mathrm{mg}, 1.0 \mathrm{mmol}, 10 \mathrm{~mol} \%)$ in Toluol $(30 \mathrm{~mL})$ wurde Boran-dimethylsulfid-Komplex $(12 \mathrm{~mL}$ einer $1 \mathrm{M}$ Lsg. in $\mathrm{CH}_{2} \mathrm{Cl}_{2}, 12 \mathrm{mmol}$ ) gegeben. Nun wurde dazu bei $45^{\circ} \mathrm{C}$ eine $1 \mathrm{M}$ Lsg. von Desoxybenzoin (72) $(2.0 \mathrm{~g}, 10.0 \mathrm{mmol})$ in THF über $1.5 \mathrm{~h}$ getropft und weitere $30 \mathrm{~min}$ gerührt. Anschließend wurde die Reaktion durch Zugabe von $\mathrm{MeOH}(2 \mathrm{~mL})$ beendet. Die Lösung wurde mit $\mathrm{NH}_{4} \mathrm{Cl}$-Lsg. $(20 \mathrm{~mL})$ gewaschen, die wässrige Phase mit $\mathrm{CH}_{2} \mathrm{Cl}_{2}(3 \times 20 \mathrm{~mL})$ extrahiert, die vereinigten organischen Phasen mit ges. NaCl-Lsg. gewaschen und über $\mathrm{Na}_{2} \mathrm{SO}_{4}$ getrocknet. Nach Entfernen des Lösungsmittels unter reduziertem Druck wurde der Alkohol $(R)-74$ (2.1 g, 10.0 mmol, quant., 84\% ee) als weißer Feststoff erhalten.

B: Zu einer Lösung des Dinitrobenzoesäureesters 75 ( $2.9 \mathrm{~g}, 7.4 \mathrm{mmol}$, >99\% ee $)$ in $\mathrm{CH}_{2} \mathrm{Cl}_{2} / \mathrm{MeOH} / \mathrm{H}_{2} \mathrm{O}$ 30:10:1 (8 mL) wurde Lithiumhydroxid-monohydrat (0.8 g, $19 \mathrm{mmol}$ ) gegeben und $1 \mathrm{~h}$ bei Umgebungstemperatur gerührt. Anschließend wurde die Lösung mit $\mathrm{CH}_{2} \mathrm{Cl}_{2}(20 \mathrm{~mL})$ verdünnt und mit $\mathrm{NaHCO}_{3}$-Lsg. $(2 \times 10 \mathrm{~mL})$ gewaschen. Die wässrige Phase wurde mit $\mathrm{CH}_{2} \mathrm{Cl}_{2}(10 \mathrm{~mL})$ extrahiert, die vereinigten organischen Phasen mit ges. NaCl-Lsg. (10 mL) gewaschen, über $\mathrm{Na}_{2} \mathrm{SO}_{4}$ getrocknet und das Lösungsmittel unter reduziertem Druck entfernt. Säulenchromatographische Reinigung an Kieselgel (P/EtOAc 20:1) lieferte den Alkohol (R)-74 (1.5 g, 7.4 mmol, quant., >99\% ee) als weißen Feststoff.

Drehwert: $[\alpha]_{D}^{20}=-50.3^{\circ}(c 1, \mathrm{EtOH})$.

Schmelzpunkt: $66^{\circ} \mathrm{C}(R), 61{ }^{\circ} \mathrm{C}(\mathrm{rac})$.

R -Wert: 0.20 (P/EtOAc 20:1).

HPLC (Chiralpak ${ }^{\circledR}$ IA , $n$-Hexan/MTBE/i-PrOH 75:24.5:0.5, $0.8 \mathrm{~mL} / \mathrm{min}, 210 \mathrm{~nm}$, $1 \mathrm{mg} / \mathrm{mL}, 8 \mu \mathrm{L}): t_{\mathrm{R}}=10.3 \min (R), 12.2(S)$. 
${ }^{1} \mathbf{H}$ NMR $\left(300 \mathrm{MHz}, \mathrm{CDCl}_{3}\right): \delta 1.99 \mathrm{ppm}(\mathrm{d}, J=2.7 \mathrm{~Hz}, 1 \mathrm{H}, \mathrm{OH}), 3.00(\mathrm{dd}, J=13.5$, $\left.8.2 \mathrm{~Hz}, 1 \mathrm{H}, 2-\mathrm{H}_{\mathrm{a}}\right), 3.07\left(\mathrm{dd}, J=13.5,5.1 \mathrm{~Hz}, 1 \mathrm{H}, 2-\mathrm{H}_{\mathrm{b}}\right), 4.92\left(\mathrm{~m}_{\mathrm{c}}, 1 \mathrm{H}, 1-\mathrm{H}\right), 7.18-$ $7.40(\mathrm{~m}, 10 \mathrm{H}, 2 \times \mathrm{Ph})$.

${ }^{13}$ C NMR $\left(75 \mathrm{MHz}, \mathrm{CDCl}_{3}\right): \delta 46.1$ ppm (C-2), 75.3 (C-1), $125.9(2 \times \mathrm{C}-\mathrm{Ph}), 126.6$ (C-Ph), 127.6 (C-Ph), 128.4 (2×C-Ph), $128.5(2 \times \mathrm{C}-\mathrm{Ph}), 129.5(2 \times \mathrm{C}-\mathrm{Ph}), 138.0$ $\left(\mathrm{C}_{i}-\mathrm{Ph}\right), 143.8\left(\mathrm{C}_{i}-\mathrm{Ph}\right)$.

IR (ATR): $\tilde{v} 3294 \mathrm{~cm}^{-1}$ (OH), 3026, 2860, 1495, 1453, 1444, 1316, 1273, 1208, 1149 , 1071, 1039, 1026, 952, 917, 778, 760, 742, 694.

UV ( $\left.\mathrm{CH}_{3} \mathrm{CN}\right): \lambda_{\max }(\lg \varepsilon) 253 \mathrm{~nm}$ (2.5516), 258 (2.6105), 264 (2.5050).

MS (EI, $70 \mathrm{eV}): m / z(\%) 77(24)[\mathrm{Ph}]^{+}, 91(29)[\mathrm{Bn}]^{+}, 92(100), 107(74)[\mathrm{M}-\mathrm{Bn}]^{+}$, $180(23)\left[\mathrm{M}-\mathrm{H}_{2} \mathrm{O}\right]^{+\cdot}, 198(3)[\mathrm{M}]^{+\cdot}$.

$\mathbf{C}_{14} \mathbf{H}_{14} \mathbf{O}(198.26)$

ber.: 198.1045, gef.: $198.1040[\mathrm{M}]^{+\cdot}(\mathrm{EI}-\mathrm{HRMS})$.

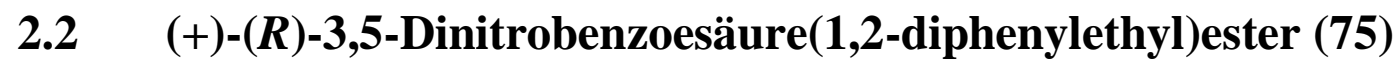<smiles>O=C(OC(Cc1ccccc1)c1ccccc1)c1cc([N+](=O)[O-])cc([N+](=O)[O-])c1</smiles>

$\mathrm{Zu}$ einer Lösung von (R)-1,2-Diphenylethanol (74) (84\% ee) (2.1 g, 10 mmol), 3,5-Dinitrobenzoylchlorid (3.0 g, $13 \mathrm{mmol})$ und DMAP (120 mg, $1.0 \mathrm{mmol}, 10 \mathrm{~mol} \%$ ) in $\mathrm{CH}_{2} \mathrm{Cl}_{2}(40 \mathrm{~mL})$ wurde bei $0{ }^{\circ} \mathrm{C} \mathrm{Et}_{3} \mathrm{~N}(2.4 \mathrm{~mL}, 17 \mathrm{mmol})$ getropft. Nach $3 \mathrm{~h}$ Rühren bei Umgebungstemperatur wurde die Lösung mit $\mathrm{CH}_{2} \mathrm{Cl}_{2}(100 \mathrm{~mL})$ verdünnt und mit ges. $\mathrm{NaHCO}_{3}$-Lsg. $(50 \mathrm{~mL})$ gewaschen. Die wässrige Phase wurde mit $\mathrm{CH}_{2} \mathrm{Cl}_{2}$ $(3 \times 50 \mathrm{~mL})$ extrahiert, die vereinigten organischen Phasen mit ges. NaCl-Lsg. $(50 \mathrm{~mL})$ gewaschen und über $\mathrm{MgSO}_{4}$ getrocknet. Nach Entfernen des Lösungmittels unter reduziertem Druck und säulenchromatographischer Reinigung an Kieselgel (Gradient: $\left.\mathrm{P} / \mathrm{CH}_{2} \mathrm{Cl}_{2} 2: 1 \rightarrow 1: 2\right)$ lieferte den Dinitrobenzoesäureester (3.8 g, $\left.9.5 \mathrm{mmol}, 95 \%\right)$ als weißen Feststoff. Umkristallisation aus $n$-Heptan/Essigester lieferte den Ester $(R)-\mathbf{7 5}$ (2.9 g, $7.4 \mathrm{mmol}, 72 \%,>99 \%$ ee). 
Drehwert: $[\alpha]_{D}^{20}=+21.0^{\circ}\left(c 0.1, \mathrm{CHCl}_{3}\right)$.

Schmelzpunkt: $107^{\circ} \mathrm{C}(R), 189^{\circ} \mathrm{C}(\mathrm{rac})$.

R -Wert: 0.40 (P/EtOAc 20:1).

${ }^{1} \mathbf{H}$ NMR $\left(300 \mathrm{MHz}, \mathrm{CDCl}_{3}\right): \delta 3.00 \mathrm{ppm}\left(\mathrm{dd}, J=14.0,5.7 \mathrm{~Hz}, 1 \mathrm{H}, 2{ }^{\prime}-\mathrm{H}_{\mathrm{a}}\right), 3.40$ (dd, $\left.J=14.0,8.4 \mathrm{~Hz}, 1 \mathrm{H}, 2^{\prime}-\mathrm{H}_{\mathrm{b}}\right), 6.22\left(\mathrm{dd}, J=8.4,5.7 \mathrm{~Hz}, 1 \mathrm{H}, 1^{\prime}-\mathrm{H}\right), 7.15-7.42(\mathrm{~m}, 10 \mathrm{H}$, $2 \times \mathrm{Ph}), 9.07(\mathrm{~d}, J=2.1 \mathrm{~Hz}, 2 \mathrm{H}, 2-\mathrm{H}, 6-\mathrm{H}), 9.18$ (t, $J=2.1 \mathrm{~Hz}, 1 \mathrm{H}, 4-\mathrm{H})$.

${ }^{13}$ C NMR (126 MHz, $\left.\mathrm{CDCl}_{3}\right): \delta 42.8$ ppm (C-2'), $75.3\left(\mathrm{C}-1^{\prime}\right), 122.3(\mathrm{C}-\mathrm{Ph}), 126.7$

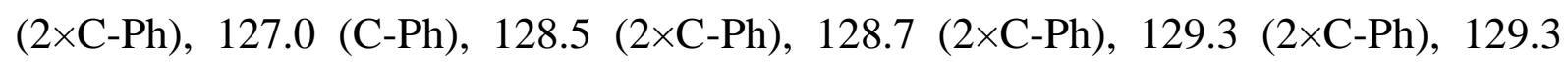
(C-2, C-6), 129.4 (C-3, C-5), $134.0\left(\mathrm{C}_{i}\right.$-Ph), 136.3 (C-1), $138.7\left(\mathrm{C}_{i}\right.$-Ph), 148.6 (C-4), $161.6(\mathrm{C}=\mathrm{O})$.

IR (Film): $\tilde{v} 3087 \mathrm{~cm}^{-1}, 1729(\mathrm{C}=\mathrm{O}), 1628,1596,1543\left(\mathrm{NO}_{2}\right), 1496,1455,1341$ $\left(\mathrm{NO}_{2}\right), 1272,1164,1074,963,945,921,905,766,752,717,699$.

UV $\left(\mathrm{CH}_{3} \mathrm{CN}\right): \lambda_{\max }(\lg \varepsilon) 208 \mathrm{~nm}(4.5501)$.

MS (ESI, $\left.\mathrm{CH}_{3} \mathrm{CN}\right): m / z(\%) 410.1(20)\left[\mathrm{M}+\mathrm{NH}_{4}\right]^{+}, 415.1(17)[\mathrm{M}+\mathrm{Na}]^{+}, 431.0(10)$ $[\mathrm{M}+\mathrm{K}]^{+}, 807.2(100)[2 \mathrm{M}+\mathrm{Na}]^{+}$.

$\begin{aligned} \mathbf{C}_{21} \mathbf{H}_{16} \mathbf{N}_{\mathbf{2}} \mathbf{O}_{6}(392.36) & \text { ber.: } 415.0901, \\ & \text { gef.: } 415.0895[\mathrm{M}+\mathrm{Na}]^{+} \text {(ESI-HRMS). }\end{aligned}$

\section{$2.3 \quad(+)-(R)-(1,2-D i p h e n y l e t h o x y)$ trimethylsilan $(60)$}

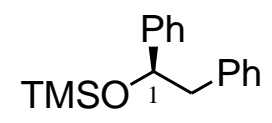

Zu einer Lösung des Alkohols (R)-74 (1.5 g, 7.4 mmol, >99\% ee) und $\mathrm{Et}_{3} \mathrm{~N}(5.7 \mathrm{~mL}$, $39.0 \mathrm{mmol})$ in $\mathrm{CH}_{2} \mathrm{Cl}_{2}(80 \mathrm{~mL})$ wurde bei $0{ }^{\circ} \mathrm{C}$ TMSOTf $(3.0 \mathrm{~mL}, 16.5 \mathrm{mmol})$ getropft. Nach $2 \mathrm{~h}$ Rühren bei Umgebungstemperatur wurde die Lösung mit $\mathrm{NH}_{4}$ Cl-Lsg. (50 mL) gewaschen, die wässrige Phase mit $\mathrm{CH}_{2} \mathrm{Cl}_{2}(3 \times 30 \mathrm{~mL})$ extrahiert, die vereinigten organischen Phasen mit ges. NaCl-Lsg. (20 mL) gewaschen, über $\mathrm{MgSO}_{4}$ getrocknet und das Lösungsmittel unter reduziertem Druck entfernt. Säulenchromatographische Reinigung an Kieselgel (PE/MTBE 80:1) lieferte den Silylether (R)-60 (1.9 g, 6.9 mmol, 93\%, >99\% ee) als farbloses Öl. 
Drehwert: $[\alpha]_{D}^{20}=+27.0^{\circ}\left(c 1, \mathrm{CHCl}_{3}\right)$.

R-Wert: 0.70 (P/EtOAc 15:1).

${ }^{1}$ H NMR $\left(300 \mathrm{MHz}, \mathrm{CDCl}_{3}\right): \delta-0.16 \mathrm{ppm}$ (s, $\left.9 \mathrm{H}, \mathrm{TMS}\right), 2.89(\mathrm{~d}, J=6.5 \mathrm{~Hz}, 2 \mathrm{H}$, 2- $\left.\mathrm{H}_{2}\right), 4.74(\mathrm{t}, J=6.5 \mathrm{~Hz}, 1 \mathrm{H}, 1-\mathrm{H}), 7.09-7.31(\mathrm{~m}, 10 \mathrm{H}, 2 \times \mathrm{Ph})$.

${ }^{13} \mathrm{C}$ NMR $\left(75 \mathrm{MHz}, \mathrm{CDCl}_{3}\right): \delta-0.3 \mathrm{ppm}$ (TMS), 47.5 (C-2), 76.4 (C-1), 125.8 $(2 \times \mathrm{C}-\mathrm{Ph}), 126.1(\mathrm{C}-\mathrm{Ph}), 127.0(\mathrm{C}-\mathrm{Ph}), 127.9(2 \times \mathrm{C}-\mathrm{Ph}), 128.0(2 \times \mathrm{C}-\mathrm{Ph}), 129.8$ (2×C-Ph), $139.0\left(\mathrm{C}_{i}-\mathrm{Ph}\right), 144.9\left(\mathrm{C}_{i}-\mathrm{Ph}\right)$.

IR (Film): $\tilde{v} 3029 \mathrm{~cm}^{-1}, 2956,1495,1454,1251,1092,943,841,758,698$.

$\mathbf{U V}\left(\mathrm{CH}_{3} \mathrm{CN}\right): \lambda_{\max }$ (lg $\left.\varepsilon\right) 252 \mathrm{~nm}$ (2.5678), 258.0 (2.6450), 264.0 (2.5313).

MS (EI, $70 \mathrm{eV}): m / z(\%) 73.1(91)[\mathrm{TMS}]^{+}, 179.2(100)[\mathrm{M}-\mathrm{Bn}]^{+}, 269.3(2)[\mathrm{M}-\mathrm{H}]^{+}$. $\mathbf{C}_{17} \mathbf{H}_{22} \mathbf{O S i}$ (270.44) ber.: 293.1332, gef.: $293.1334[\mathrm{M}+\mathrm{Na}]^{+}$(ESI-HRMS). 


\section{Synthese von (+)-(R)-4,8-Dimethylnon-7-en-4-olid}

\section{$3.1(+)-(R, R)-4,8$-Dimethyl-4-(1,2-diphenylethoxy)non-1,7-dien (77)}

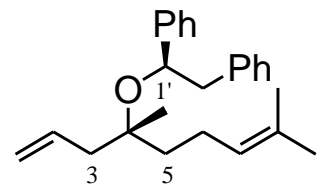

Analog zu AAV1 wurde 6-Methylhept-5-en-2-on (76) (127 mg, 1.0 mmol), PhenylBenzyl-Auxiliar (R)-60 (270 mg, $1.0 \mathrm{mmol})$, Allyltrimethylsilan (47) (125 mg, $1.1 \mathrm{mmol})$ und $\mathrm{TfOH}(20 \mu \mathrm{L}, 0.2 \mathrm{mmol}, 20 \mathrm{~mol} \%)$ in $\mathrm{CH}_{2} \mathrm{Cl}_{2}(1.0 \mathrm{~mL})$ bei $-78{ }^{\circ} \mathrm{C}$ umgesetzt. Nach Entfernen des Lösungsmittels unter reduziertem Druck wurde der Rückstand in THF $(2.0 \mathrm{~mL})$ aufgenommen und bei $0{ }^{\circ} \mathrm{C}$ mit TBAF $3 \mathrm{H}_{2} \mathrm{O}(320 \mathrm{mg}$, $1.0 \mathrm{mmol}$ ) versetzt. Nach $2 \mathrm{~h}$ Rühren bei Umgebungstemperatur wurde die Lösung über Kieselgur filtriert, mit $\mathrm{Et}_{2} \mathrm{O}(3 \times 5 \mathrm{~mL})$ gewaschen und das Lösungsmittel unter reduziertem Druck entfernt. Säulenchromatographische Reinigung an Kieselgel (PE/MTBE 100:1) lieferte den Homoallylether 77 (330 mg, 0.95 mmol, 95\%) mit einer Diastereoselektivität von 90:10 $\left({ }^{13} \mathrm{C}\right.$ NMR $)$ als ein farbloses Öl.

Drehwert: $[\alpha]_{D}^{20}=+4.7^{\circ}\left(c 1, \mathrm{CHCl}_{3}\right)$.

R-Wert: 0.40 (P/MTBE 50:1).

${ }^{1} \mathbf{H}$ NMR (300 MHz, CDCl $)$ : syn-77: $\delta 0.82$ ppm (s, $\left.3 \mathrm{H}, 4-\mathrm{CH}_{3}\right), 1.29-1.37(\mathrm{~m}, 2 \mathrm{H}$, 5- $\left.\mathrm{H}_{2}\right), 1.53\left(\mathrm{~d}, J=1.1 \mathrm{~Hz}, 3 \mathrm{H}, 9 \mathrm{a}-\mathrm{H}_{3}\right), 1.63\left(\mathrm{~d}, J=1.1 \mathrm{~Hz}, 3 \mathrm{H}, 9 \mathrm{~b}-\mathrm{H}_{3}\right), 1.78-1.99$ (m, $\left.2 \mathrm{H}, 6-\mathrm{H}_{2}\right), 2.04\left(\mathrm{dd}, J=15.4,7.3 \mathrm{~Hz}, 1 \mathrm{H}, 3-\mathrm{H}_{\mathrm{a}}\right), 2.09(\mathrm{dd}, J=15.4,7.3 \mathrm{~Hz}, 1 \mathrm{H}$, $\left.3-\mathrm{H}_{\mathrm{b}}\right), 2.83\left(\mathrm{dd}, J=13.1,5.7 \mathrm{~Hz}, 1 \mathrm{H}, 2^{\prime}-\mathrm{H}_{\mathrm{a}}\right), 2.96\left(\mathrm{dd}, J=13.1,7.5 \mathrm{~Hz}, 1 \mathrm{H}, 2^{\prime}-\mathrm{H}_{\mathrm{b}}\right)$, $4.62\left(\mathrm{dd}, J=7.5,5.7 \mathrm{~Hz}, 1 \mathrm{H}, 1^{\prime}-\mathrm{H}\right), 4.86-4.99\left(\mathrm{~m}, 3 \mathrm{H}, 1-\mathrm{H}_{2}, 7-\mathrm{H}\right), 5.64$ (ddt, $J=15.4,11.7,7.3 \mathrm{~Hz}, 1 \mathrm{H}, 2-\mathrm{H}), 7.06(\mathrm{dd}, J=7.8,1.8 \mathrm{~Hz}, 2 \mathrm{H}, 2 \times \mathrm{Ph}-\mathrm{H}), 7.12-7.29$ $(\mathrm{m}, 8 \mathrm{H}, 8 \times \mathrm{Ph}-\mathrm{H}) ;$ anti-77 (unterscheidbare Signale): $0.80\left(\mathrm{~s}, 3 \mathrm{H}, 4-\mathrm{CH}_{3}\right), 1.58$ (d, $\left.J=1.1 \mathrm{~Hz}, 3 \mathrm{H}, 9 \mathrm{a}-\mathrm{H}_{3}\right), 1.67$ (d, $J=1.1 \mathrm{~Hz}, 3 \mathrm{H}, 9 \mathrm{~b}-\mathrm{H}_{3}$ ).

${ }^{13}$ C NMR (126 MHz, CDCl 3 ): syn-77: $\delta 17.6$ ppm (C-9a), 22.4 (C-6), $23.7\left(4-\mathrm{CH}_{3}\right)$, 25.7 (C-9b), 39.5 (C-5), 43.5 (C-3), 47.1 (C-2'), 75.5 (C-1'), 77.9 (C-4), 116.8 (C-1), 124.7 (C-7), 125.9 (C-Ph), $126.3(2 \times \mathrm{C}-\mathrm{Ph}), 126.6(\mathrm{C}-\mathrm{Ph}), 127.7(2 \times \mathrm{C}-\mathrm{Ph}), 127.8$ (2×C-Ph), $129.9(2 \times C-P h), 130.9(\mathrm{C}-8), 134.9(\mathrm{C}-2), 138.7\left(\mathrm{C}_{i}-\mathrm{Ph}\right), 145.5\left(\mathrm{C}_{i}-\mathrm{Ph}\right)$; 
anti-77 (unterscheidbare Signale): 17.7 (C-9a), $23.5\left(4-\mathrm{CH}_{3}\right), 25.7$ (C-9b), 38.9 (C-5), 44.2 (C-3), 47.1 (C-2'), 75.4 (C-1'), 124.8 (C-7), 130.9 (C-8).

IR (Film): $\tilde{v} 3064 \mathrm{~cm}^{-1}, 3028,2970,2919,1639,1603,1495,1454,1377,1150,1057$, $913,758,699$.

UV $\left(\mathrm{CH}_{3} \mathrm{CN}\right): \lambda_{\max }(\lg \varepsilon) 252.5 \mathrm{~nm}$ (2.4842), 258.5 (2.5537), 264.0 (2.4224).

MS (DCI): $m / z(\%) \quad 151.2(100) \quad[\mathrm{M}-\mathrm{HOPBn}+\mathrm{H}]^{+}, 168.2(16) \quad\left[\mathrm{M}-\mathrm{HOPBn}+\mathrm{NH}_{4}\right]^{+}$, 198.2 (18) $\left[\mathrm{HOPBn}-\mathrm{H}_{2} \mathrm{O}+\mathrm{NH}_{4}\right]^{+}, 216.3(8)\left[\mathrm{HOPBn}+\mathrm{NH}_{4}\right]^{+}, 331.5(31)\left[\mathrm{M}-\mathrm{H}_{2} \mathrm{O}+\mathrm{H}\right]^{+}$, $348.5(8)\left[\mathrm{M}-\mathrm{H}_{2} \mathrm{O}+\mathrm{NH}_{4}\right]^{+}, 366.4(33)\left[\mathrm{M}+\mathrm{NH}_{4}\right]^{+}, 546.7$ (2) $\left[\mathrm{M}+\mathrm{HOPBn}-\mathrm{H}_{2} \mathrm{O}+\mathrm{NH}_{4}\right]^{+}$. $\mathbf{C}_{25} \mathbf{H}_{32} \mathbf{O}$ (348.52) ber.: 371.2345 , gef.: $371.2345[\mathrm{M}+\mathrm{Na}]^{+}$(ESI-HRMS).

\section{$3.2(+)-(R, R)-4,8-D i m e t h y l-4-(1,2-d i p h e n y l e t h o x y) n o n-7-e n-1-o l ~(78)$}

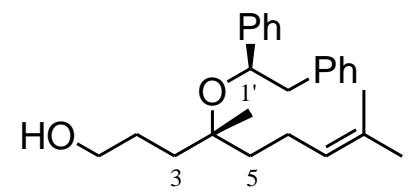

$\mathrm{Zu}$ einer Lösung des Homoallylethers 77 (235 mg, $0.67 \mathrm{mmol}$, $d r$ : 90:10) in THF (3 mL) wurde bei $0{ }^{\circ} \mathrm{C}$ 9-BBN ( $3.3 \mathrm{~mL}$ einer $0.5 \mathrm{M} \mathrm{Lsg}$. in THF, $\left.1.70 \mathrm{mmol}\right)$ getropft und die Lösung für $32 \mathrm{~h}$ bei Umgebungstemperatur gerührt. Anschließend wurde die Lösung bei $0{ }^{\circ} \mathrm{C}$ mit $3 \mathrm{~N} \mathrm{NaOH}(1.0 \mathrm{~mL})$ und $30 \%$-iger $\mathrm{H}_{2} \mathrm{O}_{2}$-Lsg. (1.0 mL) versetzt und weitere $4 \mathrm{~h}$ bei Umgebungstemperatur gerührt. Nun wurde die Reaktionslösung mit ges. $\mathrm{NH}_{4} \mathrm{Cl}-\mathrm{Lsg}$. gewaschen, die wässrige Phase mit MTBE $(3 \times 10 \mathrm{~mL})$ extrahiert, die vereinigten organischen Phasen mit ges. NaCl-Lsg. gewaschen und über $\mathrm{Na}_{2} \mathrm{SO}_{4}$ getrocknet. Nach Entfernen des Lösungsmittels unter reduziertem Druck und anschließender säulenchromatographischer Reinigung an Kieselgel $\left(\mathrm{CHCl}_{3}\right.$, 0.5\% MeOH) wurde der Alkohol 78 (212 mg, $0.61 \mathrm{mmol}, 86 \%)$ als Diastereomerengemisch im Verhältnis von ca. 90:10 ( ${ }^{13} \mathrm{C}$ NMR) in Form eines farblosen Öls erhalten. 
Drehwert: $[\alpha]_{D}^{20}=+11.2^{\circ}\left(c 1, \mathrm{CHCl}_{3}\right)$.

Rf-Wert: $0.55\left(\mathrm{CH}_{2} \mathrm{Cl}_{2}, 1 \% \mathrm{MeOH}\right)$.

${ }^{1} \mathbf{H}$ NMR $\left(300 \mathrm{MHz}, \mathrm{CDCl}_{3}\right): \underline{\text { syn-78}}: \delta 0.82 \mathrm{ppm}\left(\mathrm{s}, 3 \mathrm{H}, 4-\mathrm{CH}_{3}\right), 1.25-1.40(\mathrm{~m}, 6 \mathrm{H}$, 2- $\left.\mathrm{H}_{2}, 3-\mathrm{H}_{2}, 5-\mathrm{H}_{2}\right), 1.51\left(\mathrm{~s}, 3 \mathrm{H}, 9 \mathrm{a}-\mathrm{H}_{3}\right), 1.62$ (s, $\left.3 \mathrm{H}, 9 \mathrm{~b}-\mathrm{H}_{3}\right), 1.69-1.95\left(\mathrm{~m}, 2 \mathrm{H}, 6-\mathrm{H}_{2}\right)$, $2.82\left(\mathrm{dd}, J=13.2,5.5 \mathrm{~Hz}, 1 \mathrm{H}, 2^{\prime}-\mathrm{H}_{\mathrm{a}}\right), 2.93\left(\mathrm{dd}, J=13.2,8.0 \mathrm{~Hz}, 1 \mathrm{H}, 2^{\prime}-\mathrm{H}_{\mathrm{b}}\right), 3.42$ $\left(\mathrm{m}, 2 \mathrm{H}, 1-\mathrm{H}_{2}\right), 4.59\left(\mathrm{dd}, J=8.0,5.5 \mathrm{~Hz}, 1 \mathrm{H}, 1^{\prime}-\mathrm{H}\right), 4.88(\mathrm{t}, J=7.1 \mathrm{~Hz}, 1 \mathrm{H}, 7-\mathrm{H})$, $7.09(\mathrm{~d}, J=7.8 \mathrm{~Hz}, 2 \mathrm{H}, 2 \times \mathrm{Ph}-\mathrm{H}), 7.13-7.30(\mathrm{~m}, 8 \mathrm{H}, 8 \times \mathrm{Ph}-\mathrm{H})$; $\underline{\text { anti-78 }}$ (unterscheidbare Signale): $0.81\left(\mathrm{~s}, 3 \mathrm{H}, 4-\mathrm{CH}_{3}\right), 1.56\left(\mathrm{~s}, 3 \mathrm{H}, 9 \mathrm{a}-\mathrm{H}_{3}\right), 1.66\left(\mathrm{~s}, 3 \mathrm{H}, 9 \mathrm{~b}-\mathrm{H}_{3}\right)$, $4.96(\mathrm{t}, J=7.1 \mathrm{~Hz}, 1 \mathrm{H}, 7-\mathrm{H})$.

${ }^{13}$ C NMR (126 MHz, $\left.\mathrm{CDCl}_{3}\right)$ : $\underline{\text { syn-78}}: \delta 17.6$ ppm (C-9a), 22.7 (C-6), $23.7\left(4-\mathrm{CH}_{3}\right)$, 25.7 (C-9b), 27.0 (C-2), 34.8 (C-3), 39.3 (C-5), 47.1 (C-2'), 63.3 (C-1), 75.6 (C-1'), 78.1 (C-4), 124.5 (C-7), 125.9 (C-Ph), 126.3 (2×C-Ph), 126.7 (C-Ph), 127.8 (2×C-Ph), $127.9(2 \times \mathrm{C}-\mathrm{Ph}), 129.9(2 \times \mathrm{C}-\mathrm{Ph}), 130.9(\mathrm{C}-8), 139.0\left(\mathrm{C}_{i}-\mathrm{Ph}\right), 145.5\left(\mathrm{C}_{i}-\mathrm{Ph}\right) ; \underline{\text { anti-78}}$ (unterscheidbare Signale): 17.7 (C-9a), 22.6 (C-6), $23.8\left(4-\mathrm{CH}_{3}\right), 25.7$ (C-9b), 27.1 (C-2), 35.6 (C-3), 38.6 (C-5), 46.9 (C-2'), 63.2 (C-1), 78.1 (C-4), 124.5 (C-7), 125.9 (C-Ph), $126.4(2 \times C-P h), 126.8(\mathrm{C}-\mathrm{Ph}), 129.9(2 \times \mathrm{C}-\mathrm{Ph}), 131.0(\mathrm{C}-8), 138.6\left(\mathrm{C}_{i}-\mathrm{Ph}\right)$, $145.3\left(\mathrm{C}_{i}-\mathrm{Ph}\right)$.

IR (Film): $\tilde{v} 3356 \mathrm{~cm}^{-1}$ (OH), 3062, 3028, 2925, 2919, 1603, 1495, 1454, 1377, 1150, 1059, 913, 759, 700 .

UV ( $\left.\mathrm{CH}_{3} \mathrm{CN}\right): \lambda_{\max }(\lg \varepsilon) 252.5 \mathrm{~nm}$ (2.5851), 258.5 (2.6632), 264.0 (2.5569).

MS (ESI, MeOH): $m / z(\%) 389.2(100)[\mathrm{M}+\mathrm{Na}]^{+}$.

$\mathrm{C}_{25} \mathrm{H}_{34} \mathrm{O}_{2}(366.54)$ ber.: 389.2451, gef.: $389.2443[\mathrm{M}+\mathrm{Na}]^{+}$(ESI-HRMS). 


\section{$3.3(+)-(R, R)-4,8$-Dimethyl-4-(1,2-diphenylethoxy)non-7-en-1-al (79)}

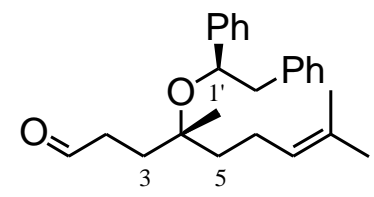

$\mathrm{Zu}$ einer Lösung des Alkohols 78 (450 mg, $1.2 \mathrm{mmol}$, $d r$ : 90:10) in $\mathrm{CH}_{2} \mathrm{Cl}_{2}(3 \mathrm{~mL})$ wurde bei $0{ }^{\circ} \mathrm{C}$ DMP (630 mg, $1.5 \mathrm{mmol}$ ) gegeben und $2 \mathrm{~h}$ bei Umgebungstemperatur gerührt. Anschließend wurde die Reaktionslösung mit Pentan auf $10 \mathrm{~mL}$ verdünnt, mit halb-ges. $\mathrm{NaHCO}_{3}$-Lsg. $(5 \times 5 \mathrm{~mL})$ extrahiert, mit ges. $\mathrm{NaCl}$-Lsg. gewaschen und über $\mathrm{Na}_{2} \mathrm{SO}_{4}$ getrocknet. Entfernen des Lösungsmittels unter reduzierten Druck lieferte den Aldehyd (460 mg, 1.2 mmol, quant.) als Diastereomerengemisch im Verhältnis von ca. 90:10 ( ${ }^{13} \mathrm{C}$ NMR) in Form eines hellgelben Öls. Dieses wurde ohne weitere Aufreinigung benutzt, da eine säulenchromatographische Reinigung an Kieselgel (P/EtOAc 40:1) etwa zu 15\% Verlust durch Zersetzung des Aldehyds führt.

Drehwert: $[\alpha]_{D}^{20}=+23.3^{\circ}\left(c 1, \mathrm{CHCl}_{3}\right)$.

R -Wert: $0.50\left(\mathrm{CH}_{2} \mathrm{Cl}_{2}\right)$.

${ }^{1} \mathrm{H}$ NMR $\left(300 \mathrm{MHz}, \mathrm{CDCl}_{3}\right)$ : syn-79: $\delta 0.77 \mathrm{ppm}\left(\mathrm{s}, 3 \mathrm{H}, 4-\mathrm{CH}_{3}\right), 1.24\left(\mathrm{~m}_{c}, 2 \mathrm{H}\right.$, 5- $\left.\mathrm{H}_{2}\right), 1.47\left(\mathrm{~s}, 3 \mathrm{H}, 9 \mathrm{a}-\mathrm{H}_{3}\right), 1.58\left(\mathrm{~m}_{c}, 2 \mathrm{H}, 3-\mathrm{H}_{2}\right), 1.59$ (s, $\left.3 \mathrm{H}, 9 \mathrm{~b}-\mathrm{H}_{3}\right), 1.77\left(\mathrm{~m}_{c}, 2 \mathrm{H}\right.$, 6- $\left.\mathrm{H}_{2}\right), 1.90-2.03\left(\mathrm{~m}, 1 \mathrm{H}, 2-\mathrm{H}_{\mathrm{a}}\right), 2.07-2.21\left(\mathrm{~m}, 1 \mathrm{H}, 2-\mathrm{H}_{\mathrm{b}}\right), 2.78(\mathrm{dd}, J=13.6,5.5 \mathrm{~Hz}$, $\left.1 \mathrm{H}, 2^{\prime}-\mathrm{H}_{\mathrm{a}}\right), 2.85\left(\mathrm{dd}, J=13.6,8.1 \mathrm{~Hz}, 1 \mathrm{H}, 2^{\prime}-\mathrm{H}_{\mathrm{b}}\right), 4.54(\mathrm{dd}, J=8.1,5.5 \mathrm{~Hz}, 1 \mathrm{H}$, $\left.1^{\prime}-\mathrm{H}\right), 4.84(\mathrm{t}, J=7.0 \mathrm{~Hz}, 1 \mathrm{H}, 7-\mathrm{H}), 7.08(\mathrm{dd}, J=7.8,1.6 \mathrm{~Hz}, 2 \mathrm{H}, 2 \times \mathrm{Ph}-\mathrm{H})$, 7.13-7.28 (m, $8 \mathrm{H}, 8 \times \mathrm{Ph}-\mathrm{H}), 9.47(\mathrm{t}, J=1.4 \mathrm{~Hz}, 1 \mathrm{H}, 1-\mathrm{H})$; anti-79 (unterscheidbare Signale): 1.53 (s, $\left.3 \mathrm{H}, 9 \mathrm{a}-\mathrm{H}_{3}\right), 1.65\left(\mathrm{~s}, 3 \mathrm{H}, 9 \mathrm{~b}-\mathrm{H}_{3}\right), 4.91(\mathrm{t}, J=7.0 \mathrm{~Hz}, 1 \mathrm{H}, 7-\mathrm{H})$.

${ }^{13} \mathrm{C}$ NMR (126 MHz, $\mathrm{CDCl}_{3}$ ): $\underline{\text { syn-79: } \delta} 17.6$ ppm (C-9a), 22.8 (C-6), $23.7\left(4-\mathrm{CH}_{3}\right)$, 25.7 (C-9b), 30.4 (C-3), 38.5 (C-2), 39.4 (C-5), 47.1 (C-2'), 75.8 (C-1'), 77.3 (C-4), 124.2 (C-7), $126.0(\mathrm{C}-\mathrm{Ph}), 126.2(2 \times \mathrm{C}-\mathrm{Ph}), 126.8(\mathrm{C}-\mathrm{Ph}), 127.9(2 \times \mathrm{C}-\mathrm{Ph}), 128.0$ (2×C-Ph), $129.8(2 \times \mathrm{C}-\mathrm{Ph}), 131.2(\mathrm{C}-8), 139.0\left(\mathrm{C}_{i}-\mathrm{Ph}\right), 145.5\left(\mathrm{C}_{i}-\mathrm{Ph}\right), 202.5(\mathrm{CHO})$; anti-79 (unterscheidbare Signale): 17.7 (C-9a), 22.7 (C-6), 25.7 (C-9b), 31.2 (C-3), 38.6 (C-2), 38.7 (C-5), 46.8 (C-2'), 77.3 (C-4), 124.3 (C-7), 126.0 (C-Ph), 126.4 $(2 \times \mathrm{C}-\mathrm{Ph}), 126.9(\mathrm{C}-\mathrm{Ph}), 127.8(2 \times \mathrm{C}-\mathrm{Ph}), 131.2(\mathrm{C}-8), 138.6\left(\mathrm{C}_{i}-\mathrm{Ph}\right), 145.2\left(\mathrm{C}_{i}-\mathrm{Ph}\right)$, 202.6 (CHO). 
IR (Film): $\tilde{v} 3062 \mathrm{~cm}^{-1}$, 3028, 2921, 2719, 1724 (C=O), 1603, 1495, 1454, 1378, 1118, 1059, 941, 912, 759, 701 .

UV $\left(\mathrm{CH}_{3} \mathrm{CN}\right): \lambda_{\max }(\lg \varepsilon) 253$ (2.6276), 258 (2.6762), 264 (2.5580).

MS (ESI, MeOH): m/z (\%) 387.2 (6) $[\mathrm{M}+\mathrm{Na}]^{+}, 419.3$ (100) $[\mathrm{M}+\mathrm{MeOH}+\mathrm{Na}]^{+}, 815.5$

(28) $[2(\mathrm{M}+\mathrm{MeOH})+\mathrm{Na}]^{+}$.

$\mathbf{C}_{25} \mathbf{H}_{32} \mathbf{O}_{2}$ (364.52). $\quad$ ber.: 387.2295,

gef.: $387.2290[\mathrm{M}+\mathrm{Na}]^{+}$(ESI-HRMS).

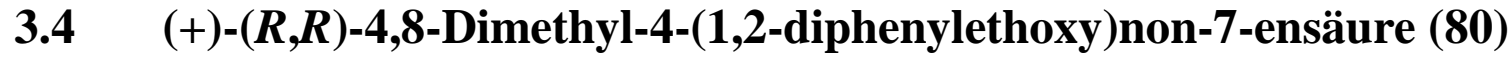

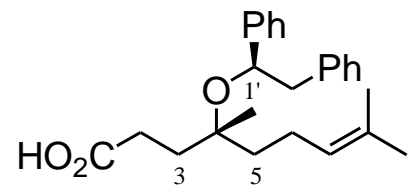

$\mathrm{Zu}$ einer Lösung des rohen Aldehyds 79 (72 mg, $0.20 \mathrm{mmol}, \quad d r$ : 90:10) und 2-Methyl-2-buten $(0.4 \mathrm{~mL}, 4.0 \mathrm{mmol})$ in DMF (1 mL) wurde $1.3 \mathrm{M} \mathrm{NaH}_{2} \mathrm{PO}_{4}$-Lsg. $(0.35 \mathrm{~mL})$ und $\mathrm{NaClO}_{2}(72 \mathrm{mg}, 0.80 \mathrm{mmol})$ gegeben. Nach $2 \mathrm{~h}$ Rühren bei Umgebungstemperatur wurde die Reaktionslösung mit $1 \mathrm{~N}$ HCl-Lsg. (1 mL) angesäuert, mit MTBE $(3 \times 5 \mathrm{~mL})$ extrahiert, die vereinigten organischen Phasen mit $\mathrm{HCl}$ angesäuerter ges. NaCl-Lsg. $(5 \times 2 \mathrm{~mL}, \mathrm{pH}=1)$ gewaschen, über $\mathrm{Na}_{2} \mathrm{SO}_{4}$ getrocknet und das Lösungsmittels unter reduziertem Druck entfernt. Nach säulenchromatographischer Reinigung an Kieselgel $\left(\mathrm{CH}_{2} \mathrm{Cl}_{2}, 1 \% \mathrm{AcOH}\right)$ wurde die Säure 80 (46 mg, $0.12 \mathrm{mmol}, 60 \%$ ) als Diastereomerengemisch im Verhältnis von ca. 90:10 $\left({ }^{13} \mathrm{C}\right.$ NMR) in Form eines farblosen Öls erhalten.

Drehwert: $[\alpha]_{D}^{20}=+15.3^{\circ}\left(c 1, \mathrm{CHCl}_{3}\right)$.

Rf-Wert: $0.13\left(\mathrm{CH}_{2} \mathrm{Cl}_{2}, 1 \% \mathrm{AcOH}\right)$.

${ }^{1} \mathbf{H}$ NMR $\left(300 \mathrm{MHz}, \mathrm{CDCl}_{3}\right): \underline{\text { syn-80}}: \delta 0.79 \mathrm{ppm}\left(\mathrm{s}, 3 \mathrm{H}, 4-\mathrm{CH}_{3}\right), 1.27\left(\mathrm{~m}_{c}, 2 \mathrm{H}\right.$, 5- $\left.\mathrm{H}_{2}\right), 1.48\left(\mathrm{~d}, J=1.0 \mathrm{~Hz}, 3 \mathrm{H}, 9 \mathrm{a}-\mathrm{H}_{3}\right), 1.60\left(\mathrm{~d}, J=1.0 \mathrm{~Hz}, 3 \mathrm{H}, 9 \mathrm{~b}-\mathrm{H}_{3}\right), 1.68$ (t, $\left.J=7.6 \mathrm{~Hz}, 2 \mathrm{H}, 3-\mathrm{H}_{2}\right), 1.72-1.92\left(\mathrm{~m}, 2 \mathrm{H}, 6-\mathrm{H}_{2}\right), 2.14(\mathrm{dt}, J=16.6,7.6 \mathrm{~Hz}, 1 \mathrm{H}$, $\left.2-\mathrm{H}_{\mathrm{a}}\right), 2.23\left(\mathrm{dt}, J=16.6,7.6 \mathrm{~Hz}, 1 \mathrm{H}, 2-\mathrm{H}_{\mathrm{b}}\right), 2.83\left(\mathrm{dd}, J=13.6,5.6 \mathrm{~Hz}, 1 \mathrm{H}, 2^{\prime}-\mathrm{H}_{\mathrm{a}}\right)$, $2.92\left(\mathrm{dd}, J=13.6,8.0 \mathrm{~Hz}, 1 \mathrm{H}, 2^{\prime}-\mathrm{H}_{\mathrm{b}}\right), 4.60\left(\mathrm{dd}, J=8.0,5.6 \mathrm{~Hz}, 1 \mathrm{H}, 1^{\prime}-\mathrm{H}\right), 4.82$ (tsep, $J=7.0,1.0 \mathrm{~Hz}, 1 \mathrm{H}, 7-\mathrm{H}), 7.10(\mathrm{dd}, J=7.8,1.8 \mathrm{~Hz}, 2 \mathrm{H}, 2 \times \mathrm{Ph}-\mathrm{H}), 7.15-7.32(\mathrm{~m}, 8 \mathrm{H}$, 
8xPh-H); anti-80 (unterscheidbare Signale): $0.81\left(\mathrm{~s}, 3 \mathrm{H}, 4-\mathrm{CH}_{3}\right), 1.55(\mathrm{~d}, J=1.0 \mathrm{~Hz}$, $\left.3 \mathrm{H}, 9 \mathrm{a}-\mathrm{H}_{3}\right), 1.66\left(\mathrm{~d}, J=1.0 \mathrm{~Hz}, 3 \mathrm{H}, 9 \mathrm{~b}-\mathrm{H}_{3}\right), 4.92$ (tsep, $\left.J=7.0,1.0 \mathrm{~Hz}, 1 \mathrm{H}, 7-\mathrm{H}\right)$.

${ }^{13}$ C NMR (126 MHz, $\left.\mathrm{CD}_{2} \mathrm{Cl}_{2}\right): \underline{\text { syn-80: }} \delta 17.6$ ppm (C-9a), $23.1\left(4-\mathrm{CH}_{3}\right), 23.3(\mathrm{C}-6)$, 25.7 (C-9b), 29.0 (C-3), 33.6 (C-2), 39.5 (C-5), 47.2 (C-2'), 75.9 (C-1'), 77.8 (C-4), 124.5 (C-7), 126.3 (C-Ph), 126.6 (2×C-Ph), 127.1 (C-Ph), 128.1 (2×C-Ph), 128.2 (2×C-Ph), 130.1 (2×C-Ph), $131.4(\mathrm{C}-8), 139.1\left(\mathrm{C}_{i}-\mathrm{Ph}\right), 145.7\left(\mathrm{C}_{i}-\mathrm{Ph}\right), 180.3\left(\mathrm{CO}_{2} \mathrm{H}\right)$; anti-80 (unterscheidbare Signale): 17.7 (C-9a), $23.0\left(4-\mathrm{CH}_{3}\right), 23.7$ (C-6), 25.7 (C-9b), 30.1 (C-3), 34.2 (C-2), 38.6 (C-5), 47.0 (C-2'), 75.8 (C-1'), 124.6 (C-7), 126.3 (C-Ph), $126.7(2 \times \mathrm{C}-\mathrm{Ph}), 127.1(\mathrm{C}-\mathrm{Ph}), 128.1(2 \times \mathrm{C}-\mathrm{Ph})$.

IR (ATR): $\tilde{v} 3428 \mathrm{~cm}^{-1}$ (OH), 3061, 3028, 2971, 2932, 1706 (C=O), 1603, 1495, 1453, 1378, 1284, 1153, 1052, 944, 912, 759, 738, 698.

UV ( $\left.\mathrm{CH}_{3} \mathrm{CN}\right): \lambda_{\max }(\lg \varepsilon) 252 \mathrm{~nm}$ (2.8876), 259 (2.8712).

MS (ESI, MeOH): $m / z(\%) 379.2(100)[\mathrm{M}-\mathrm{H}]^{-}$.

$\mathbf{C}_{25} \mathbf{H}_{32} \mathbf{O}_{3}(380.52) \quad$ ber.: 379.2279 , gef.: $379.2285[\mathrm{M}-\mathrm{H}]^{-}$(ESI-HRMS).

\section{5 rac-2-Methyl-2-(4-methylpent-3-enyl)oxiran (83)}

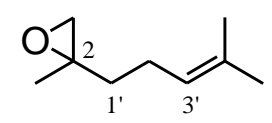

Eine Suspension von Natriumhydrid $(220 \mathrm{mg}$ eines 60\%-igen Gemischs in Parafin, $5.5 \mathrm{mmol})$ in DMSO $(20 \mathrm{~mL})$ wurde $1 \mathrm{~h}$ bei $60^{\circ} \mathrm{C}$ gerührt. Anschließend wurde bei Umgebungstemperatur Trimethylsulfoxoniumiodid (1.20 g, $5.5 \mathrm{mmol})$ zugegeben und weitere $30 \mathrm{~min}$ bis zur vollständigen Auflösung gerührt. Zu der hellgelben Lösung wurde dann 6-Methylhept-5-en-2-on (76) $(0.74 \mathrm{~mL}, 5.0 \mathrm{mmol})$ gegeben und $2 \mathrm{~h}$ bei Umgebungstemperatur gerührt. Anschließend wurde die Lösung mit gesättigter NaCl-Lsg. (10 mL) versetzt und mit MTBE $(2 \times 20 \mathrm{~mL})$ extrahiert. Die vereinigten organischen Phasen wurden mit gesättigter NaCl-Lsg. $(5 \times 10 \mathrm{~mL})$ gewaschen, über $\mathrm{Na}_{2} \mathrm{SO}_{4}$ getrocknet und das Lösungsmittel unter verminderten Druck ( $p>250$ mbar)

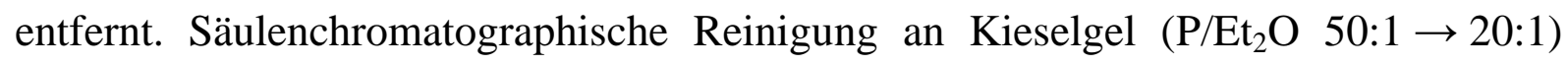
lieferte das Epoxid 83 (365 mg, 2.6 mmol, 52\%) als farblose Flüssigkeit. ${ }^{[42 \mathrm{~b}]}$ 
R -Wert: 0.29 (PE/EtOAc 10:1).

${ }^{1} \mathbf{H}$ NMR $\left(300 \mathrm{MHz}, \mathrm{CDCl}_{3}\right): \delta 1.26$ ppm (s, $\left.3 \mathrm{H}, 2-\mathrm{CH}_{3}\right), 1.40-1.62\left(\mathrm{~m}, 2 \mathrm{H}, 1^{\prime}-\mathrm{H}_{2}\right)$, $1.55\left(\mathrm{~s}, 3 \mathrm{H}, 5^{\prime} \mathrm{a}-\mathrm{H}_{3}\right), 1.63\left(\mathrm{~s}, 3 \mathrm{H}, 5^{\prime} \mathrm{b}-\mathrm{H}_{3}\right), 2.02\left(\mathrm{q}, J=7.7 \mathrm{~Hz}, 2 \mathrm{H}, 2^{\prime}-\mathrm{H}_{2}\right), 2.50$ $\left(\mathrm{d}, J=4.9 \mathrm{~Hz}, 1 \mathrm{H}, 3-\mathrm{H}_{\mathrm{a}}\right), 2.56\left(\mathrm{~d}, J=4.9 \mathrm{~Hz}, 1 \mathrm{H}, 3-\mathrm{H}_{\mathrm{b}}\right), 5.04\left(\mathrm{~m}_{\mathrm{c}}, 1 \mathrm{H}, 3^{\prime}-\mathrm{H}\right)$.

${ }^{13}$ C NMR (126 MHz, $\left.\mathrm{CDCl}_{3}\right): \delta 17.6$ ppm (C-5'a), $20.9\left(2-\mathrm{CH}_{3}\right), 23.8\left(\mathrm{C}-2^{\prime}\right), 25.6$ (C-5'b), $36.8\left(\mathrm{C}-1^{\prime}\right), 53.8$ (C-3), 56.7 (C-2), 123.5 (C-3'), 131.7 (C-4').

IR (ATR): $\tilde{v} 2965 \mathrm{~cm}^{-1}, 2923,2856,1449,1389,1376,1267,1108,1072,902,834$, 795.

MS (EI, $70 \mathrm{eV}): m / z(\%) 140.1$ (3) [M] ${ }^{+*}, 109.1$ (76), 82.0 (48), 69.1 (57), $67.0(90)$, $55.0(36), 43.0(31), 41.0(100)$.

$\mathbf{C}_{9} \mathbf{H}_{16} \mathrm{O}(140.22)$

ber.: 163.1093, gef.: $163.1094[\mathrm{M}+\mathrm{Na}]^{+}(\mathrm{ESI}-\mathrm{HRMS})$.

\section{6 (+)-4,8-Dimethylnon-7-en-4-olid (82)}

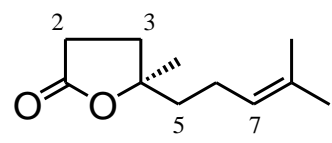

A: $\mathrm{Zu}$ einer Lösung von Malonsäuredimethylester $(0.63 \mathrm{~mL}, 5.5 \mathrm{mmol})$ in EtOH (4 mL) wurde Natrium-methanolat (1.0 mL einer 30\%-igen Lsg. in $\mathrm{MeOH}, 5.5 \mathrm{mmol}$ ) gegeben. Die resultierende Natrium-dimethylmalonat-Lsg. wurde über $30 \mathrm{~min}$ bei $70{ }^{\circ} \mathrm{C} \mathrm{zu}$ einer Lösung des Epoxids rac-83 (700 mg, $\left.5.0 \mathrm{mmol}\right)$ in EtOH $(5 \mathrm{~mL})$ getropft und für $4 \mathrm{~h}$ unter Rückfluss erhitzt. Anschließend wurde die Lösung unter reduziertem Druck auf etwa das halbe Volumen $(\sim 5 \mathrm{~mL})$, eingeengt, mit 10\%-iger $\mathrm{NaOH}(4.0 \mathrm{~mL}, 11.0 \mathrm{mmol})$ versetzt und für weitere $1.5 \mathrm{~h}$ unter Rückfluss erhitzt. Die Reaktionslösung wurde direkt auf Eiswasser $(40 \mathrm{~mL})$ gegeben und mit $3 \mathrm{~N} \mathrm{HCl}$ $(6.0 \mathrm{~mL}, 18.0 \mathrm{mmol})$ auf $\mathrm{pH} \leq 2$ angesäuert. Die wässrige Phase wurde mit MTBE $(4 \times 20 \mathrm{~mL})$ extrahiert, die vereinigten organischen Phasen über $\mathrm{Na}_{2} \mathrm{SO}_{4}$ getrocknet und das Lösungsmittel unter reduziertem Druck ( $\mathrm{p} \geq 50$ mbar) entfernt. Abschließend wurde der erhaltene Rückstand unter einer Argonatmosphäre für $3 \mathrm{~h}$ bei $130{ }^{\circ} \mathrm{C}$ erhitzt. Saülenchromatographische Reinigung des Rückstands an Kieselgel $\left(\mathrm{CHCl}_{3}\right.$, 0.4\% Aceton) lieferte das $\gamma$-Lacton rac-82 (630 mg, $3.5 \mathrm{mmol}, 63 \%)$ als farblose Flüssigkeit (Lit. $\left.{ }^{[42]}: 71 \%\right)$. 
B: $\mathrm{Zu}$ einer tiefblauen Lösung von Lithium $(8.0 \mathrm{mg}, 1.1 \mathrm{mmol})$ in flüssigem Ammoniak wurde bei $-78^{\circ} \mathrm{C}$ eine Lösung der Säure $(R, R)-80(24 \mathrm{mg}, 63 \mu \mathrm{mol}, d r$ : 20:10) in $\mathrm{Et}_{2} \mathrm{O}(1 \mathrm{~mL})$ gegeben. Nach $20 \mathrm{~min}$ Rühren bei $-78{ }^{\circ} \mathrm{C}$ wurde bis zur vollständigen Entfärbung der Lösung $\mathrm{MeOH}$ zugegeben. Durch Entfernen der Kühlung lies man den Ammoniak verdampfen (Dauer: ca. 3-4 h, Vorsicht: Siedeverzüge). Nach Entfernen des Lösungsmittels wurde zu einer Lösung des Rückstands in $\mathrm{Et}_{2} \mathrm{O}(10 \mathrm{~mL})$ bei $0{ }^{\circ} \mathrm{C}$ wasserfreies $\mathrm{K}_{2} \mathrm{CO}_{3}(20 \mathrm{mg}, 145 \mu \mathrm{mol})$ und Methyliodid (5 $\mu \mathrm{L}, 75 \mu \mathrm{mol})$ gegeben und 30 min bei Umgebungstemperatur gerührt. Die Reaktion wurde durch Zugabe von MeOH (2 mL) beendet, die Lösung mit $\mathrm{CH}_{2} \mathrm{Cl}_{2}(3 \times 20 \mathrm{~mL})$ extrahiert, die vereinigten organischen Phasen mit ges. NaCl-Lsg. (10 mL) gewaschen und das Lösungsmittelgemisch $\left(\mathrm{CH}_{2} \mathrm{Cl}_{2} / \mathrm{MeOH}\right)$ unter verminderten Druck ( $\mathrm{p} \geq 400$ mbar) azeotrop entfernt. Saülenchromatographische Reinigung an Kieselgel $\left(\mathrm{CHCl}_{3}, 0.4 \%\right.$ Aceton) lieferte das $\gamma$-Lacton $(R)-82(7.8 \mathrm{mg}, 43 \mu \mathrm{mol}, 68 \%, 72 \%$ ee $)$ als farblose Flüssigkeit.

Drehwert: $[\alpha]_{D}^{20}=+5.1^{\circ}\left(c 1, \mathrm{CHCl}_{3}\right)$; Lit. $^{[42]}:+6.3^{\circ}\left(\right.$ c 3.0, $\mathrm{CHCl}_{3}, 90 \%$ ee $)$.

R -Wert: $0.24\left(\mathrm{CH}_{2} \mathrm{Cl}_{2}\right), 0.15$ (PE/MTBE 5:1).

GC (OV1701 mit 50\% Heptakis(6- $O$-TBDMS-2,3-di- $O$-methyl) $\beta$-cyclodextrin, $\mathrm{H}_{2}$ $\left.140{ }^{\circ} \mathrm{C}, 50 \mathrm{kPa}\right): t_{\mathrm{R}}=9.86 \mathrm{~min}(R$-Enantiomer $), 10.45$ ( $S$-Enantiomer $)$.

${ }^{1} \mathbf{H}$ NMR $\left(300 \mathrm{MHz}, \mathrm{CDCl}_{3}\right): \delta 1.35$ ppm (s, $\left.3 \mathrm{H}, 4-\mathrm{CH}_{3}\right), 1.56$ (s, $\left.3 \mathrm{H}, 9 \mathrm{a}-\mathrm{H}_{3}\right), 1.64$ (d, $\left.J=1.1 \mathrm{~Hz}, 3 \mathrm{H}, 9 \mathrm{~b}-\mathrm{H}_{3}\right), 1.60-1.68\left(\mathrm{~m}, 2 \mathrm{H}, 5-\mathrm{H}_{2}\right), 1.94(\mathrm{ddd}, J=12.8,9.2,6.7 \mathrm{~Hz}$, $1 \mathrm{H}, 3-\mathrm{H}_{\mathrm{a}}$ ), 1.97-2.07 (m, $\left.2 \mathrm{H}, 6-\mathrm{H}_{2}\right), 2.07$ (ddd, $J=12.8,9.2,8.3 \mathrm{~Hz}, 1 \mathrm{H}, 3-\mathrm{H}_{\mathrm{b}}$ ), 2.52 (ddd, $\left.J=18.0,9.0,6.7 \mathrm{~Hz}, 1 \mathrm{H}, 2-\mathrm{H}_{\mathrm{a}}\right), 2.59\left(\mathrm{ddd}, J=18.0,9.2,8.3 \mathrm{~Hz}, 1 \mathrm{H}, 2-\mathrm{H}_{\mathrm{b}}\right)$, 5.04 (tsep, $J=7.1,1.4 \mathrm{~Hz}, 1 \mathrm{H}, 7-\mathrm{H}$ ).

${ }^{13}$ C NMR (126 MHz, $\left.\mathrm{CDCl}_{3}\right): \delta 17.7$ ppm (C-9a), 22.6 (C-6), 25.6 (4-CH $\left.3, \mathrm{C}-9 \mathrm{~b}\right)$, 29.1 (C-2), 33.0 (C-3), 40.9 (C-5), 86.6 (C-4), 123.0 (C-7), 132.2 (C-8), 176.5 (C=O). IR (ATR): $\tilde{v} 2971 \mathrm{~cm}^{-1}, 2926,2858,1764(\mathrm{C}=\mathrm{O}), 1453,1381,1282,1258,1202$, $1167,1142,1106,1082,953,932,899$.

MS (ESI, MeOH): $m / z(\%) 205.1(60)[\mathrm{M}+\mathrm{Na}]^{+}, 387.2(100)[2 \mathrm{M}+\mathrm{Na}]^{+}$.

$\mathbf{C}_{\mathbf{1 1}} \mathbf{H}_{18} \mathbf{O}_{\mathbf{2}}(182.26) \quad$ ber.: 205.1199, gef.: 205.1201 [M+Na $]^{+}$(ESI-HRMS). 


\section{Tietze-Allylierung von Ketoestern}

\subsection{Lävulinsäure-iso-propylester (86b)}<smiles>CCOC(=O)CCC(C)=O</smiles>

$\mathrm{Zu}$ einer Lösung von Lävulinsäure $(1.0 \mathrm{~mL}, 10 \mathrm{mmol})$ in $\mathrm{CH}_{2} \mathrm{Cl}_{2}(20 \mathrm{~mL})$ wurde bei $0{ }^{\circ} \mathrm{C}$ Diisopropylcarbodiimid $(1.7 \mathrm{~mL}, 11 \mathrm{mmol})$ gegeben. Nach $5 \mathrm{~min}$ Rühren bei Umgebungstemperatur wurde $i$-PrOH $(4.0 \mathrm{~mL}, 50 \mathrm{mmol})$ und DMAP $(60 \mathrm{mg}$, $0.5 \mathrm{mmol}, 5 \mathrm{~mol} \%$ ) bei $0{ }^{\circ} \mathrm{C}$ zugegeben und anschließend $1.5 \mathrm{~h}$ bei dieser Temperatur gerührt. Anschließend wurde Kieselgel (5 g) zugegeben und das Lösungsmittel unter verminderten Druck ( $p>150$ mbar) entfernt. Filtration über Kieselgel $(\mathrm{P} \rightarrow \mathrm{P} / \mathrm{MTBE}$ 4:1) und anschließende Destillation unter reduzierten Druck lieferte den Ketoester 86b $(1.3 \mathrm{~g}, 8.4 \mathrm{mmol}, 84 \%)$ als farblose Flüssigkeit.

Siedepunkt: $48^{\circ} \mathrm{C}(1.0$ mbar $)$.

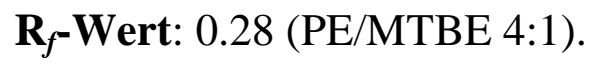

${ }^{1} \mathrm{H}$ NMR $\left(300 \mathrm{MHz}, \mathrm{CDCl}_{3}\right): \delta 1.16 \mathrm{ppm}\left(\mathrm{d}, J=6.3 \mathrm{~Hz}, 6 \mathrm{H}, \mathrm{CH}\left(\mathrm{CH}_{3}\right)_{2}\right), 2.12(\mathrm{~s}, 3 \mathrm{H}$, $\left.\mathrm{CH}_{3} \mathrm{C}(\mathrm{O})\right), 2.46\left(\mathrm{t}, J=6.5 \mathrm{~Hz}, 2 \mathrm{H}, \mathrm{CH}_{2} \mathrm{CO}_{2} i-\mathrm{Pr}\right), 2.67$ (t, $J=6.5 \mathrm{~Hz}, 2 \mathrm{H}, \mathrm{C}(\mathrm{O}) \mathrm{CH}_{2}$ ), 4.92 (sep, $\left.J=6.3 \mathrm{~Hz}, 1 \mathrm{H}, \mathrm{CH}\left(\mathrm{CH}_{3}\right)_{2}\right)$.

${ }^{13} \mathbf{C}$ NMR $\left(76 \mathrm{MHz}, \quad \mathrm{CDCl}_{3}\right): \delta 21.7 \mathrm{ppm}\left(\mathrm{CH}\left(\mathrm{CH}_{3}\right)_{2}\right), \quad 28.3 \quad\left(\mathrm{CH}_{2} \mathrm{CO}_{2} i-\mathrm{Pr}\right), \quad 29.8$ $\left(\mathrm{CH}_{3} \mathrm{C}(\mathrm{O})\right), 37.9\left(\mathrm{C}(\mathrm{O}) \mathrm{CH}_{2}\right), 67.8\left(\mathrm{CH}\left(\mathrm{CH}_{3}\right)_{2}\right), 172.1\left(\mathrm{CO}_{2} i-\mathrm{Pr}\right), 206.6(\mathrm{C}=\mathrm{O})$.

IR (ATR): $\tilde{v} 2981 \mathrm{~cm}^{-1}, 2937,1716$ (C=O), 1409, 1372, 1159, 1107, 935.

MS (ESI, MeOH) $m / z(\%) 181.1(58)[\mathrm{M}+\mathrm{Na}]^{+}, 339.2(100)[2 \mathrm{M}+\mathrm{Na}]^{+}$.

$\mathrm{C}_{8} \mathrm{H}_{14} \mathrm{O}_{3}(158.20)$ ber.: 181.0835 , gef.: $181.0836[\mathrm{M}+\mathrm{Na}]^{+}$(ESI-HRMS). 


\subsection{Lävulinsäure-tert-butylester (86c)}

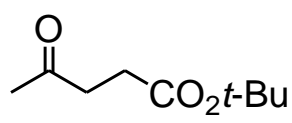

$\mathrm{Zu}$ einer Lösung von Lävulinsäure (2.6 mL, $25 \mathrm{mmol})$ in $\mathrm{CH}_{2} \mathrm{Cl}_{2}(40 \mathrm{~mL})$ wurde bei $0{ }^{\circ} \mathrm{C}$ Diisopropylcarbodiimid $(4.2 \mathrm{~mL}, 27 \mathrm{mmol})$ gegeben. Nach $5 \mathrm{~min}$ Rühren bei Umgebungstemperatur wurde $t-\mathrm{BuOH}(12 \mathrm{~mL}, 120 \mathrm{mmol})$ und DMAP $(150 \mathrm{mg}$, $1.2 \mathrm{mmol}, 5 \mathrm{~mol} \%$ ) bei $0{ }^{\circ} \mathrm{C}$ zugegeben und dann bei $40{ }^{\circ} \mathrm{C}$ für $2.5 \mathrm{~h}$ gerührt. Anschließend wurde Kieselgel (12 g) zugegeben und das Lösungsmittel unter reduziertem Druck ( $p>150$ mbar) entfernt. Filtration über Kieselgel (P/MTBE 10:1 $\rightarrow$ 4:1) und anschließende Destillation unter reduziertem Druck lieferte den Ketoester 86c (2.3 g, $13 \mathrm{mmol}, 52 \%)$ als farblose Flüssigkeit.

Siedepunkt: $50{ }^{\circ} \mathrm{C}(1 \mathrm{mbar})$

R f $^{\text {-Wert: }} 0.32$ (PE/MTBE 2:1).

${ }^{1} \mathbf{H}$ NMR (300 MHz, $\left.\mathrm{CDCl}_{3}\right): \delta 1.39 \mathrm{ppm}\left(\mathrm{s}, 9 \mathrm{H}, t\right.$-Bu), $2.14\left(\mathrm{~s}, 3 \mathrm{H}, \mathrm{CH}_{3} \mathrm{C}(\mathrm{O})\right), 2.44$ $\left(\mathrm{t}, J=6.6 \mathrm{~Hz}, 2 \mathrm{H}, \mathrm{CH}_{2} \mathrm{CO}_{2} t-\mathrm{Bu}\right), 2.64\left(\mathrm{t}, J=6.6 \mathrm{~Hz}, 2 \mathrm{H}, \mathrm{C}(\mathrm{O}) \mathrm{CH}_{2}\right)$.

${ }^{13} \mathbf{C ~ N M R}\left(126 \mathrm{MHz}, \quad \mathrm{CDCl}_{3}\right): \delta 28.0 \mathrm{ppm}\left(\mathrm{C}\left(\mathrm{CH}_{3}\right)_{3}\right), 29.1 \quad\left(\mathrm{CH}_{2} \mathrm{CO}_{2} t-\mathrm{Bu}\right), 29.8$ $\left(\mathrm{CH}_{3} \mathrm{C}(\mathrm{O})\right), 38.0\left(\mathrm{C}(\mathrm{O}) \mathrm{CH}_{2}\right), 80.5\left(C\left(\mathrm{CH}_{3}\right)_{3}\right), 171.9\left(\mathrm{CO}_{2} t-\mathrm{Bu}\right), 206.9(\mathrm{C}=\mathrm{O})$.

IR (ATR): $\tilde{v} 2978 \mathrm{~cm}^{-1}, 1717,1363,1240,1144,857$.

MS (ESI, MeOH): m/z (\%) $195.1(54)[\mathrm{M}+\mathrm{Na}]^{+}, 367.2(100)[2 \mathrm{M}+\mathrm{Na}]^{+}$.

$\mathrm{C}_{9} \mathrm{H}_{16} \mathrm{O}_{3}(172.22)$ ber.: 195.0992, gef.: $195.0992[\mathrm{M}+\mathrm{Na}]^{+}$(ESI-HRMS). 


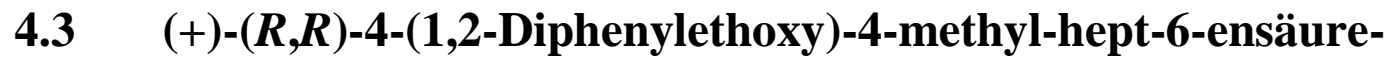 methylester $(89 \mathrm{a})$}

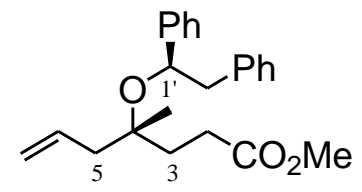

Analog zu AAV1 wurde Lävulinsäuremethylester (86a) (390 mg, 3.0 mmol), PhenylBenzyl-Auxiliar (R)-60 (810 mg, $3.0 \mathrm{mmol}$ ), Allyltrimethylsilan (47) (410 mg, $3.6 \mathrm{mmol})$ und $\mathrm{TfOH}(60 \mu \mathrm{L}, 20 \mathrm{~mol} \%)$ in $\mathrm{CH}_{2} \mathrm{Cl}_{2}(1.5 \mathrm{~mL})$ bei $-78^{\circ} \mathrm{C}$ umgesetzt. Säulenchromatographische Reinigung an Kieselgel (PE/MTBE 50:1 $\rightarrow$ 20:1) lieferte den Homoallylether 89a (960 mg, 2.7 mmol, 91\%) mit einer Diastereoselektivität von 94:6 ( ${ }^{13} \mathrm{C}$ NMR) als ein farbloses Öl.

Drehwert: $[\alpha]_{D}^{20}=+29.9^{\circ}\left(c 1, \mathrm{CHCl}_{3}\right)$.

R -Wert: 0.15 (PE/MTBE 30:1).

${ }^{1}$ H NMR $\left(300 \mathrm{MHz}, \mathrm{CDCl}_{3}\right)$ ： syn-89a: $\delta 0.82 \mathrm{ppm} \quad\left(\mathrm{s}, 3 \mathrm{H}, 4-\mathrm{CH}_{3}\right), 1.66$ (ddd, $\left.J=14.2,9.6,6.3 \mathrm{~Hz}, 1 \mathrm{H}, 3-\mathrm{H}_{\mathrm{a}}\right), 1.74\left(\mathrm{ddd}, J=14.2,9.3,6.6 \mathrm{~Hz}, 1 \mathrm{H}, 3-\mathrm{H}_{\mathrm{b}}\right), 1.94$ (dd br., $J=13.9,7.4 \mathrm{~Hz}, 1 \mathrm{H}, 5-\mathrm{H}_{\mathrm{a}}$ ), 2.07 (dd br., $J=13.9,7.2 \mathrm{~Hz}, 1 \mathrm{H}, 5-\mathrm{H}_{\mathrm{b}}$ ), 2.23 (ddd, $J=16.1,9.3,6.3 \mathrm{~Hz}, 1 \mathrm{H}, 2-\mathrm{H}_{\mathrm{a}}$ ), 2.30 (ddd, $J=16.1,9.6,6.6 \mathrm{~Hz}, 1 \mathrm{H}, 2-\mathrm{H}_{\mathrm{b}}$ ), $2.83\left(\mathrm{dd}, J=13.2,5.4 \mathrm{~Hz}, 1 \mathrm{H}, 2^{\prime}-\mathrm{H}_{\mathrm{a}}\right), 2.95\left(\mathrm{dd}, J=13.2,7.8 \mathrm{~Hz}, 1 \mathrm{H}, 2^{\prime}-\mathrm{H}_{\mathrm{b}}\right), 3.63$ (s, $3 \mathrm{H}, \mathrm{OMe}$ ), 4.62 (dd, $\left.J=7.8,5.4 \mathrm{~Hz}, 1 \mathrm{H}, 1^{\prime}-\mathrm{H}\right), 4.91-5.00\left(\mathrm{~m}, 2 \mathrm{H}, 7-\mathrm{H}_{2}\right), 5.58$ (ddt, $J=16.7,10.4,7.3 \mathrm{~Hz}, 1 \mathrm{H}, 6-\mathrm{H}), 7.07-7.31(\mathrm{~m}, 10 \mathrm{H}, 2 \times \mathrm{Ph}$ ); anti-89a (unterscheidbare Signale): 0.79 (s, $\left.3 \mathrm{H}, 4-\mathrm{CH}_{3}\right), 2.19\left(\mathrm{~m}_{\mathrm{c}}, 2 \mathrm{H}, 2-\mathrm{H}_{2}\right), 3.66(\mathrm{~s}, 3 \mathrm{H}, \mathrm{OMe})$, 4.87-4.96 (m, 2 H, 7- $\mathrm{H}_{2}$ ), 5.61 (ddt, $J=16.7,10.4,7.3 \mathrm{~Hz}, 1 \mathrm{H}, 6-\mathrm{H}$ ).

${ }^{13} \mathbf{C}$ NMR (126 MHz, $\left.\mathrm{CDCl}_{3}\right)$ : syn-89a: $\delta 23.6$ ppm (4-CH $)$, $28.5(\mathrm{C}-2), 34.2(\mathrm{C}-3)$, 43.2 (C-5), 46.9 (C-2'), 51.4 (OMe), 75.7 (C-1'), 77.1 (C-4), 117.4 (C-7), 126.0 (C-Ph), $126.2(2 \times \mathrm{C}-\mathrm{Ph}), 126.8(\mathrm{C}-\mathrm{Ph}), 127.8(2 \times \mathrm{C}-\mathrm{Ph}), 127.9(2 \times \mathrm{C}-\mathrm{Ph}), 129.8$ $(2 \times \mathrm{C}-\mathrm{Ph}), 134.1(\mathrm{C}-6), 138.6\left(\mathrm{C}_{i}-\mathrm{Ph}\right), 145.2\left(\mathrm{C}_{i}-\mathrm{Ph}\right), 174.3(\mathrm{C}=\mathrm{O})$; anti-89a (unterscheidbare Signale): 23.1 (4- $\left.\mathrm{CH}_{3}\right), 28.3$ (C-2), 33.6 (C-3), 44.2 (C-5), 47.0 (C-2'), 77.1 (C-4), $117.4(\mathrm{C}-7), 126.1$ (2×C-Ph), $127.8(2 \times \mathrm{C}-\mathrm{Ph}), 138.7\left(\mathrm{C}_{i}-\mathrm{Ph}\right), 145.3\left(\mathrm{C}_{i}-\mathrm{Ph}\right)$, $174.3(\mathrm{C}=\mathrm{O})$. 
IR (Film): $\tilde{v} 3064 \mathrm{~cm}^{-1}, 3028,2948,1739(\mathrm{C}=\mathrm{O}), 1639(\mathrm{C}=\mathrm{C}), 1495,1454,1379$, 1308, 1172, 1057, 916, 759 .

UV $\left(\mathrm{CH}_{3} \mathrm{CN}\right): \lambda_{\max }(\lg \varepsilon) 253.0 \mathrm{~nm}$ (2.5510), 258.5 (2.6314), 264.0 (2.5107).

MS (ESI, MeOH): $m / z(\%) 370.2(22)\left[\mathrm{M}+\mathrm{NH}_{4}\right]^{+}, 375.2(100)[\mathrm{M}+\mathrm{Na}]^{+}$.

$\mathbf{C}_{23} \mathbf{H}_{28} \mathbf{O}_{3}(352.47)$ ber.: 375.1931 , gef.: $375.1934[\mathrm{M}+\mathrm{Na}]^{+}(\mathrm{ESI}-\mathrm{HRMS})$.

\section{4 (+)-(R,R)-4-(1,2-Diphenylethoxy)-4-methyl-hept-6-ensäure- iso-propylester $(89 \mathrm{~b})$}

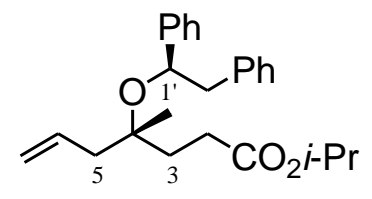

Analog zu AAV1 wurde Lävulinsäureisopropylester (86b) (270 mg, 1.7 mmol), Phenyl-Benzyl-Auxiliar (R)-60 (470 mg, 1.7 mmol), Allyltrimethylsilan (47) (220 mg, $1.9 \mathrm{mmol})$ und $\mathrm{TfOH}(35 \mu \mathrm{L}, 20 \mathrm{~mol} \%)$ in $\mathrm{CH}_{2} \mathrm{Cl}_{2}(1.5 \mathrm{~mL})$ bei $-78{ }^{\circ} \mathrm{C}$ umgesetzt. Säulenchromatographische Reinigung an Kieselgel (PE/MTBE 50:1 $\rightarrow$ 20:1) lieferte den Homoallylether 89b (625 mg, 1.6 mmol, 94\%) mit einer Diastereoselektivität von 94:6 $\left({ }^{13} \mathrm{C}\right.$ NMR) als ein farbloses Öl.

Drehwert: $[\alpha]_{D}^{24}=+25.0^{\circ}\left(c 1, \mathrm{CHCl}_{3}\right)$.

Rf-Wert: 0.24 (PE/MTBE 20:1).

${ }^{1} \mathbf{H}$ NMR $\left(300 \mathrm{MHz}, \mathrm{CDCl}_{3}\right): \underline{s y n-89 b}: \delta 0.79$ ppm $\left(\mathrm{s}, 3 \mathrm{H}, 4-\mathrm{CH}_{3}\right), 1.18,1.20(2 \times \mathrm{d}$, $\left.J=6.3 \mathrm{~Hz}, 3 \mathrm{H}, \mathrm{CH}\left(\mathrm{CH}_{3}\right)_{2}\right), 1.56-1.75$ (m, $\left.2 \mathrm{H}, 3-\mathrm{H}_{2}\right), 1.93$ (dd br., $J=14.0,7.4 \mathrm{~Hz}$, $1 \mathrm{H}, 5-\mathrm{H}_{\mathrm{a}}$ ), 2.04 (dd br., $J=14.0,7.3 \mathrm{~Hz}, 1 \mathrm{H}, 5-\mathrm{H}_{\mathrm{b}}$ ), 2.09-2.27 (m, $2 \mathrm{H}, 2-\mathrm{H}_{2}$ ), 2.80 $\left(\mathrm{dd}, J=13.2,5.5 \mathrm{~Hz}, 1 \mathrm{H}, 2^{\prime}-\mathrm{H}_{\mathrm{a}}\right), 2.93\left(\mathrm{dd}, J=13.2,7.8 \mathrm{~Hz}, 1 \mathrm{H}, 2^{\prime}-\mathrm{H}_{\mathrm{b}}\right), 4.59$ (dd, J=7.8, $\left.5.5 \mathrm{~Hz}, 1 \mathrm{H}, 1^{\prime}-\mathrm{H}\right), 4.88-4.98\left(\mathrm{~m}, 3 \mathrm{H}, 7-\mathrm{H}_{2}, \mathrm{CH}\left(\mathrm{CH}_{3}\right)_{2}\right), 5.57$ (ddt,

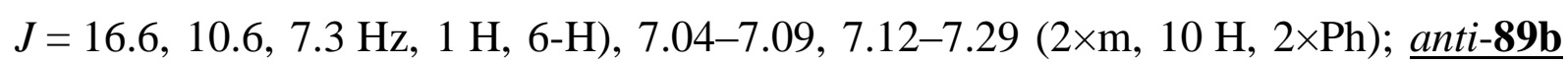
(unterscheidbare Signale): $0.77\left(\mathrm{~s}, 4-\mathrm{CH}_{3}\right), 1.22,1.23\left(2 \times \mathrm{d}, J=6.3 \mathrm{~Hz}, \mathrm{CH}\left(\mathrm{CH}_{3}\right)_{2}\right)$. 
${ }^{13} \mathbf{C}$ NMR $\left(126 \mathrm{MHz}, \mathrm{CDCl}_{3}\right): \underline{s y n-89 \mathbf{b}}: \delta 21.9$ ppm $\left(\mathrm{CH}\left(\mathrm{CH}_{3}\right)_{2}\right), 23.6\left(4-\mathrm{CH}_{3}\right), 29.1$ (C-2), 34.2 (C-3), 43.4 (C-5), $46.9\left(\mathrm{C}-2^{\prime}\right), 67.3\left(\mathrm{CH}\left(\mathrm{CH}_{3}\right)_{2}\right), 75.7\left(\mathrm{C}-1^{\prime}\right), 77.2(\mathrm{C}-4)$, 117.3 (C-7), 126.0 (C-Ph), $126.3(2 \times \mathrm{C}-\mathrm{Ph}), 126.8(\mathrm{C}-\mathrm{Ph}), 127.8(2 \times \mathrm{C}-\mathrm{Ph}), 127.9$ (2×C-Ph), $129.9(2 \times C-P h), 134.2(\mathrm{C}-6), 138.7\left(\mathrm{C}_{i}-\mathrm{Ph}\right), 145.3\left(\mathrm{C}_{i}-\mathrm{Ph}\right), 173.4(\mathrm{C}=\mathrm{O})$; anti-89b (unterscheidbare Signale): $21.9\left(\mathrm{CH}\left(\mathrm{CH}_{3}\right)_{2}\right), 23.2\left(4-\mathrm{CH}_{3}\right), 28.9(\mathrm{C}-2), 33.6$ (C-3), 44.2 (C-5), 47.1 (C-2'), 77.2 (C-4), 117.3 (C-7), 126.2 (C-Ph), 126.2 (2×C-Ph), $127.8(2 \times \mathrm{C}-\mathrm{Ph}), 138.7\left(\mathrm{C}_{i}-\mathrm{Ph}\right), 145.3\left(\mathrm{C}_{i}-\mathrm{Ph}\right), 173.4(\mathrm{C}=\mathrm{O})$.

IR (ATR): $\tilde{v} 2978 \mathrm{~cm}^{-1}, 2935,1727(\mathrm{C}=\mathrm{O}), 1638,1603,1495,1453,1375,1297$, 1177, 1107, 1054, 912, 757, 698.

UV $\left(\mathrm{CH}_{3} \mathrm{CN}\right): \lambda_{\max }(\lg \varepsilon) 254 \mathrm{~nm}(2.6499), 259$ (2.7153).

MS (ESI, MeOH): $m / z(\%) 381.2(3)[\mathrm{M}+\mathrm{H}]^{+}, 398.3(23)\left[\mathrm{M}+\mathrm{NH}_{4}\right]^{+}, 403.2(100)$ $[\mathrm{M}+\mathrm{Na}]^{+}, 419.2(6)[\mathrm{M}+\mathrm{K}]^{+}$.

$\mathbf{C}_{25} \mathbf{H}_{32} \mathbf{O}_{3}$ (380.50) ber.: 403.2244, gef.: $403.2244[\mathrm{M}+\mathrm{Na}]^{+}$(ESI-HRMS).

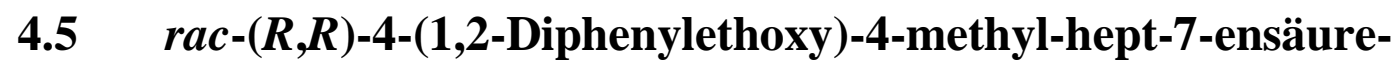 tert-butylester (89c)}

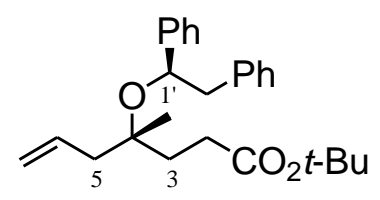

Analog zu AAV1 wurde Lävulinsäure-tert-butylester (86c) (170 mg, 1.0 mmol), Phenyl-Benzyl-Auxiliar rac-60 (270 mg, 1.0 mmol), Allyltrimethylsilan (47) (125 mg, $1.1 \mathrm{mmol})$ und TfOH $(20 \mu \mathrm{L}, 20 \mathrm{~mol} \%)$ in $\mathrm{CH}_{2} \mathrm{Cl}_{2}(1.0 \mathrm{~mL})$ bei $-78^{\circ} \mathrm{C}$ umgesetzt. Säulenchromatographische Reinigung an Kieselgel (PE/MTBE 80:1 $\rightarrow$ 40:1) lieferte den Homoallylether 89c (260 mg, $1.6 \mathrm{mmol}, 66 \%$ ) mit einer Diastereoselektivität von 93:7 ( ${ }^{13} \mathrm{C}$ NMR) als ein farbloses Öl. 
R -Wert: 0.22 (PE/MTBE 20:1).

${ }^{1}$ H NMR (300 MHz, $\left.\mathrm{CDCl}_{3}\right)$ : syn-89c: $\delta 0.78 \mathrm{ppm}\left(\mathrm{s}, 3 \mathrm{H}, 4-\mathrm{CH}_{3}\right), 1.40(\mathrm{~s}, 9 \mathrm{H}, t-\mathrm{Bu})$, 1.53-1.72 (m, $2 \mathrm{H}, 3-\mathrm{H}_{2}$ ), 1.92 (dd br., $J=13.8,7.3 \mathrm{~Hz}, 1 \mathrm{H}, 5-\mathrm{H}_{\mathrm{a}}$ ), 2.04 (dd br., $\left.J=13.8,7.3 \mathrm{~Hz}, 1 \mathrm{H}, 5-\mathrm{H}_{\mathrm{b}}\right), 2.03-2.22\left(\mathrm{~m}, 2 \mathrm{H}, 2-\mathrm{H}_{2}\right), 2.80(\mathrm{dd}, J=13.2,5.6 \mathrm{~Hz}, 1 \mathrm{H}$, $\left.2^{\prime}-\mathrm{H}_{\mathrm{a}}\right), 2.93\left(\mathrm{dd}, J=13.2,7.8 \mathrm{~Hz}, 1 \mathrm{H}, 2^{\prime}-\mathrm{H}_{\mathrm{b}}\right), 4.59\left(\mathrm{dd}, J=7.8,5.6 \mathrm{~Hz}, 1 \mathrm{H}, 1^{\prime}-\mathrm{H}\right)$, 4.88-4.97 (m, $2 \mathrm{H}, 7-\mathrm{H}_{2}$ ), 5.57 (ddt, $J=16.5,10.4,7.3 \mathrm{~Hz}, 1 \mathrm{H}, 6-\mathrm{H}$ ), 7.03-7.27 $(\mathrm{m}, 10 \mathrm{H}, 2 \times \mathrm{Ph}) ; \underline{\text { anti-89c }}$ (unterscheidbare Signale): $0.77\left(\mathrm{~s}, 4-\mathrm{CH}_{3}\right), 1.43(\mathrm{~s}, t-\mathrm{Bu})$.

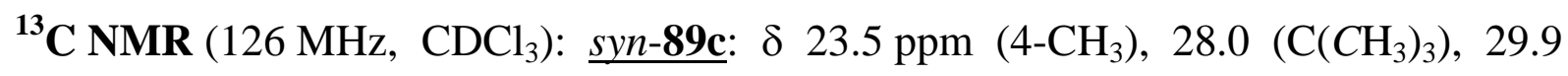

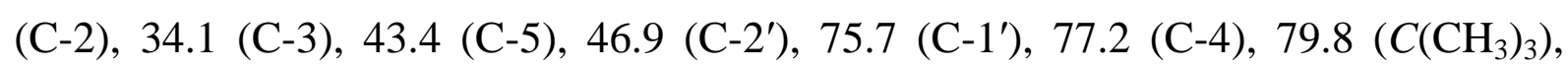
117.3 (C-7), 126.1 (C-Ph), $126.3(2 \times \mathrm{C}-\mathrm{Ph}), 126.8(\mathrm{C}-\mathrm{Ph}), 127.9(2 \times \mathrm{C}-\mathrm{Ph}), 128.0$ (2×C-Ph), $129.9(2 \times C-P h), 134.4(\mathrm{C}-6), 138.8\left(\mathrm{C}_{i}-\mathrm{Ph}\right), 145.4\left(\mathrm{C}_{i}-\mathrm{Ph}\right), 173.4(\mathrm{C}=\mathrm{O})$; anti-89c (unterscheidbare Signale): $23.2\left(4-\mathrm{CH}_{3}\right), 28.1\left(\mathrm{C}\left(\mathrm{CH}_{3}\right)_{3}\right), 29.7(\mathrm{C}-2), 33.6$ (C-3), 44.2 (C-5), 47.0 (C-2'), 75.7 (C-1'), 117.4 (C-7), 126.3 (2×C-Ph), 127.9 (2×C-Ph), $138.8\left(\mathrm{C}_{i}-\mathrm{Ph}\right), 145.5\left(\mathrm{C}_{i}-\mathrm{Ph}\right), 173.4(\mathrm{C}=\mathrm{O})$.

IR (ATR): $\tilde{v} 3063 \mathrm{~cm}^{-1}, 3028,2976,2931,1727(\mathrm{C}=\mathrm{O}), 1638,1602,1495,1454$, 1366, 1147, 1054, 913, 846, 757, 698.

$\mathbf{U V}\left(\mathrm{CH}_{3} \mathrm{CN}\right): \lambda_{\max }(\lg \varepsilon) 254 \mathrm{~nm}$ (3.3889), 259 (3.4807).

MS (ESI, MeOH): $m / z(\%) 395.3(3)[\mathrm{M}+\mathrm{H}]^{+}, 412.3(16)\left[\mathrm{M}+\mathrm{NH}_{4}\right]^{+}, 417.2(100)$ $[\mathrm{M}+\mathrm{Na}]^{+}, 433.2(13)[\mathrm{M}+\mathrm{K}]^{+}$.

$\mathbf{C}_{26} \mathbf{H}_{34} \mathbf{O}_{3}$ (394.55)

ber.: 417.2400, gef.: $417.2397[\mathrm{M}+\mathrm{Na}]^{+}$(ESI-HRMS). 


\section{6 rac-(R,R)-5-(1,2-Diphenylethoxy)-5-methyl-oct-7-ensäure- methylester (90)}

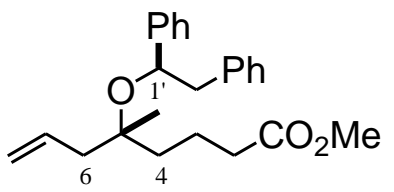

Analog zu AAV1 wurde 5-Oxohexansäuremethylester (87) (145 mg, $1.0 \mathrm{mmol}$ ), Phenyl-Benzyl-Auxiliar rac-60 (270 mg, 1.0 mmol), Allyltrimethylsilan (47) (125 mg, $1.1 \mathrm{mmol})$ und $\mathrm{TfOH}(20 \mu \mathrm{L}, 20 \mathrm{~mol} \%)$ in $\mathrm{CH}_{2} \mathrm{Cl}_{2}(1.0 \mathrm{~mL})$ bei $-78{ }^{\circ} \mathrm{C}$ umgesetzt. Säulenchromatographische Reinigung an Kieselgel (PE/MTBE 50:1 $\rightarrow$ 20:1) lieferte den Homoallylether 89c (360 mg, 1.0 mmol, 98\%) mit einer Diastereoselektivität von 91:9 $\left({ }^{13} \mathrm{C}\right.$ NMR $)$ als ein farbloses Öl.

R -Wert: 0.22 (PE/MTBE 20:1).

${ }^{1}$ H NMR $\left(300 \mathrm{MHz}, \mathrm{CDCl}_{3}\right): \underline{\text { syn-90}}: \delta 0.79$ ppm (s, $\left.3 \mathrm{H}, 5-\mathrm{CH}_{3}\right), 1.17-1.36(\mathrm{~m}, 2 \mathrm{H}$, 4- $\left.\mathrm{H}_{2}\right), 1.41-1.58\left(\mathrm{~m}, 2 \mathrm{H}, 3-\mathrm{H}_{2}\right), 1.97-2.11\left(\mathrm{~m}, 4 \mathrm{H}, 2-\mathrm{H}_{2}, 6-\mathrm{H}_{2}\right), 2.80$ (dd, $J=13.2$, $\left.5.6 \mathrm{~Hz}, 1 \mathrm{H}, 2^{\prime}-\mathrm{H}_{\mathrm{a}}\right), 2.94\left(\mathrm{dd}, J=13.2,7.8 \mathrm{~Hz}, 1 \mathrm{H}, 2^{\prime}-\mathrm{H}_{\mathrm{b}}\right), 3.62$ (s, $\left.3 \mathrm{H}, \mathrm{OMe}\right), 4.59$ (dd, $\left.J=7.8,5.6 \mathrm{~Hz}, 1 \mathrm{H}, 1^{\prime}-\mathrm{H}\right), 4.89-4.97$ (m, $2 \mathrm{H}, 8-\mathrm{H}_{2}$ ), 5.60 (ddt, $J=16.0,11.2$, $7.3 \mathrm{~Hz}, 1 \mathrm{H}, 7-\mathrm{H}), 7.04-7.28$ (m, $10 \mathrm{H}, 2 \times \mathrm{Ph}$ ); anti-90 (unterscheidbare Signale): 3.65 (s, $3 \mathrm{H}, \mathrm{OMe}$ ).

${ }^{13} \mathbf{C}$ NMR (126 MHz, $\left.\mathrm{CDCl}_{3}\right): \underline{\text { syn-90}}: \delta 19.2$ ppm (C-3), $23.7\left(5-\mathrm{CH}_{3}\right), 34.3(\mathrm{C}-2)$, 38.8 (C-4), 43.3 (C-6), 46.9 (C-2'), 51.4 (OMe), 75.6 (C-1'), 77.8 (C-5), 117.0 (C-8),

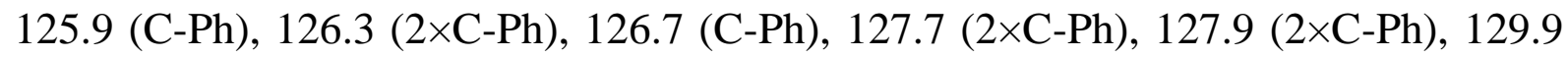
(2×C-Ph), $134.6(\mathrm{C}-7), 138.7\left(\mathrm{C}_{i}-\mathrm{Ph}\right), 145.4\left(\mathrm{C}_{i}-\mathrm{Ph}\right), 173.9(\mathrm{C}=\mathrm{O})$; anti-90 (unterscheidbare Signale): 19.1 (C-3), 23.5 (5- $\mathrm{CH}_{3}$ ), 34.4 (C-2), 38.2 (C-4), 44.1 (C-6), 47.1 (C-2'), $51.4(\mathrm{OMe}), 138.8\left(\mathrm{C}_{i}-\mathrm{Ph}\right), 145.5\left(\mathrm{C}_{i}-\mathrm{Ph}\right)$.

IR (ATR): $\tilde{v} 3063 \mathrm{~cm}^{-1}, 3028,2948,1737(\mathrm{C}=\mathrm{O}), 1638,1603,1495,1453,1435$, 1376, 1194, 1170, 1054, 912, 758, 698.

UV $\left(\mathrm{CH}_{3} \mathrm{CN}\right): \lambda_{\max }(\lg \varepsilon) 253 \mathrm{~nm}$ (2.7415), 259 (2.7742).

MS (ESI, MeOH): $m / z(\%) 389.2(100)[\mathrm{M}+\mathrm{Na}]^{+}$.

$\mathbf{C}_{24} \mathrm{H}_{30} \mathrm{O}_{3}$ (366.49). ber.: 389.2087, gef.: 389.2089 [M+Na] ${ }^{+}$(ESI-HRMS). 


\section{Synthese von $(+)-(R)$-Pinnatolid}

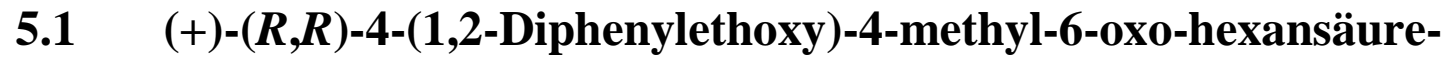 methylester (95)}

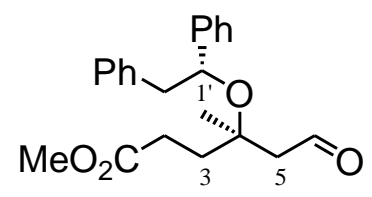

In eine Lösung des Homoallylethers 89a (880 mg, $2.5 \mathrm{mmol}$ ) in $\mathrm{CH}_{2} \mathrm{Cl}_{2} / \mathrm{MeOH}$ 10:1 $\left(55 \mathrm{~mL}\right.$ ) wurde bei $-78{ }^{\circ} \mathrm{C}$ bis zur Sättigung Ozon eingeleitet (erkennbar an hellblauer Färbung). Anschließend wurde bis zur Entfärbung Argon durch die Lösung geleitet und Triphenylphosphin (860 mg, $3.3 \mathrm{mmol}$ ) zugegeben. Nach Aufwärmen der Lösung auf Raumtemperatur über $14 \mathrm{~h}$ wurde das Lösungsmittel unter reduziertem Druck entfernt. Säulenfiltration über Kieselgel (PE/MTBE 20:1 $\rightarrow$ 3:1) lieferte den Aldehyd 95 (870 mg, $2.5 \mathrm{mmol}, 98 \%$ ) als falblosen Feststoff.

Drehwert: $[\alpha]_{D}^{20}=+22.7^{\circ}\left(c 1, \mathrm{CHCl}_{3}\right)$.

R-Wert: 0.26 (PE/MTBE 5:1).

${ }^{1} \mathbf{H}$ NMR (300 MHz, $\mathrm{CDCl}_{3}$ ): $\underline{\text { syn-95: }} \delta 0.89$ ppm (s, $3 \mathrm{H}, 4-\mathrm{CH}_{3}$ ), 1.69 (ddd, $J=14.3$, 9.4, $6.3 \mathrm{~Hz}, 1 \mathrm{H}, 3-\mathrm{H}_{\mathrm{a}}$ ), 1.77 (ddd, $J=14.3,9.0,6.6 \mathrm{~Hz}, 1 \mathrm{H}, 3-\mathrm{H}_{\mathrm{b}}$ ), 2.08 (dd, $J=15.1$, $\left.2.9 \mathrm{~Hz}, 1 \mathrm{H}, 5-\mathrm{H}_{\mathrm{a}}\right), 2.16\left(\mathrm{~m}_{\mathrm{c}}, 2 \mathrm{H}, 2-\mathrm{H}_{2}\right), 2.25\left(\mathrm{dd}, J=15.1,2.9 \mathrm{~Hz}, 1 \mathrm{H}, 5-\mathrm{H}_{\mathrm{b}}\right), 2.81$ (dd, $\left.J=13.4,5.1 \mathrm{~Hz}, 1 \mathrm{H}, 2^{\prime}-\mathrm{H}_{\mathrm{a}}\right), 2.88$ (dd, $J=13.4,8.3 \mathrm{~Hz}, 1 \mathrm{H}, 2^{\prime}-\mathrm{H}_{\mathrm{b}}$ ), 3.57 (s, $3 \mathrm{H}$, OMe), $4.58\left(\mathrm{dd}, J=8.3,5.1 \mathrm{~Hz}, 1 \mathrm{H}, 1^{\prime}-\mathrm{H}\right), 7.08-7.30(\mathrm{~m}, 10 \mathrm{H}, 2 \times \mathrm{Ph}), 9.36$ (t, $J=2.9 \mathrm{~Hz}, 1 \mathrm{H}, \mathrm{CHO}$ ); anti-95 (unterscheidbare Signale): 3.61 (s, OMe), 9.56 (t, $J=2.9 \mathrm{~Hz}, \mathrm{CHO})$.

${ }^{13}$ C NMR (126 MHz, $\left.\mathrm{CDCl}_{3}\right)$ : syn-95: $\delta 24.0$ ppm (4- $\left.\mathrm{CH}_{3}\right), 28.4$ (C-2), $34.9(\mathrm{C}-3)$, 46.6 (C-2'), 51.5 (OMe), 51.9 (C-5), 76.3, 76.5 (C-4, C-1'), 126.1 (2×C-Ph), 126.3 (C-Ph), $127.2(\mathrm{C}-\mathrm{Ph}), 128.0(2 \times \mathrm{C}-\mathrm{Ph}), 128.2(2 \times \mathrm{C}-\mathrm{Ph}), 129.7(2 \times \mathrm{C}-\mathrm{Ph}), 138.4$ $\left(\mathrm{C}_{i}-\mathrm{Ph}\right), 144.5\left(\mathrm{C}_{i}-\mathrm{Ph}\right), 173.5(\mathrm{C}=\mathrm{O}), 201.6(\mathrm{CHO})$; anti-95 (unterscheidbare Signale): 28.2 (C-2), 34.3 (C-3), 46.7 (C-2'), 52.6 (C-5), 76.1 (C-4, C-1'), 126.2 (C-Ph), 127.2 (C-Ph), $128.0(2 \times \mathrm{C}-\mathrm{Ph}), 128.3(2 \times \mathrm{C}-\mathrm{Ph}), 138.4\left(\mathrm{C}_{i}-\mathrm{Ph}\right), 144.5\left(\mathrm{C}_{i}-\mathrm{Ph}\right)$.

IR (ATR): $\tilde{v} 3061 \mathrm{~cm}^{-1}, 3028,2947,2842,1731,1718(2 \times \mathrm{C}=\mathrm{O}), 1603,1496,1453$, 1436, 1381, 1307, 1192, 1170, 1112, 1053, 985, 959, 915, 759, 699. 
UV $\left(\mathrm{CH}_{3} \mathrm{CN}\right): \lambda_{\max }(\lg \varepsilon) 253 \mathrm{~nm}$ (2.6991), 259 (2.7369).

MS (DCI): $m / z(\%) 372.3(100)\left[\mathrm{M}+\mathrm{NH}_{4}\right]^{+}, 726.4(3)\left[2 \mathrm{M}+\mathrm{NH}_{4}\right]^{+}$.

MS (ESI, $\mathrm{MeOH}): m / z(\%) 377.2(32)[\mathrm{M}+\mathrm{Na}]^{+}, 409.2(100)[\mathrm{M}+\mathrm{MeOH}+\mathrm{Na}]^{+}, 795.4$

(2) $[2(\mathrm{M}+\mathrm{MeOH})+\mathrm{Na}]^{+}$.

$\mathbf{C}_{22} \mathbf{H}_{26} \mathbf{O}_{4}(354.44) \quad$ ber.: 377.1723 ,

gef.: $377.1732[\mathrm{M}+\mathrm{Na}]^{+}(\mathrm{ESI}-\mathrm{HRMS})$.

\section{2 (+)-(R,R)-4-(1,2-Diphenylethoxy)-6-hydroxy-4-methyl-hexansäure- methylester (96)}

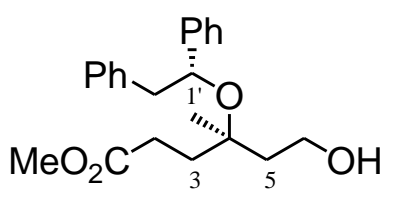

A: $\mathrm{Zu}$ einer Lösung des rohen Aldehyds 95 (870 mg, $2.45 \mathrm{mmol}, d r: 95: 5)$ in $\mathrm{MeOH}$ $(20 \mathrm{~mL})$ wurde bei $0{ }^{\circ} \mathrm{C}$ langsam portionsweise $\mathrm{NaBH}_{4}(110 \mathrm{mg}, 2.9 \mathrm{mmol})$ gegeben. Nach Abklingen der Gasentwicklung wurde die Kühlung entfernt und $30 \mathrm{~min}$ bei Umgebungstemperatur gerührt. Anschließend wurde die Reaktion durch Zugabe von $\mathrm{NH}_{4} \mathrm{Cl}(100 \mathrm{mg})$ beendet und die Lösung unter reduziertem Druck auf die Hälfte eingeengt. Nach Zugabe von $\mathrm{H}_{2} \mathrm{O}(5 \mathrm{~mL})$ wurde mit MTBE $(3 \times 15 \mathrm{~mL})$ extrahiert, die vereinigten organischen Phasen mit ges. NaCl-Lsg. (5 mL) gewaschen und über $\mathrm{Na}_{2} \mathrm{SO}_{4}$ getrocknet. Säulenchromatographische Reinigung an Kieselgel (PE/MTBE 4:5) lieferte den Alkohol 96 (860 mg, $2.40 \mathrm{mmol}, 98 \%, d r$ : 95:5) als farblosen Feststoff.

B: $\mathrm{Zu}$ einer Lösung des Dinitrobenzoesäureesters 97 (1.4 g, $2.5 \mathrm{mmol}, d r$ : >99:1) in $\mathrm{CH}_{2} \mathrm{Cl}_{2} / \mathrm{MeOH}$ 1:1 (100 mL) wurde Lithiumhydroxid-monohydrat (10 mg, $0.24 \mathrm{mmol}$, 10 mol\%) gegeben. Nach 1 h Rühren wurde die Reaktion durch Zugabe von $\mathrm{NaHCO}_{3}$ Lsg. (10 mL) beendet. Die wässrige Phase wurde mit $\mathrm{CH}_{2} \mathrm{Cl}_{2}(3 \times 15 \mathrm{~mL})$ extrahiert, die vereinigten organischen Phasen mit ges. NaCl-Lsg. gewaschen, über $\mathrm{Na}_{2} \mathrm{SO}_{4}$ getrocknet und anschließend das Lösungsmittel unter reduziertem Druck entfernt. Säulenchromatographische Reinigung an Kieselgel $\left(\mathrm{CH}_{2} \mathrm{Cl}_{2}, 1 \% \mathrm{MeOH}\right)$ lieferte den Alkohol 96 (0.9 g, 2.5 mmol, quant., $d r$ : >99:1) als farbloser Feststoff. 
Drehwert: $[\alpha]_{D}^{23}=+6.9^{\circ}\left(c 1, \mathrm{CHCl}_{3}\right)$.

Schmelzpunkt: $62{ }^{\circ} \mathrm{C}(d r:>99: 1)$.

R-Wert: 0.22 (PE/MTBE 1:1), $0.12\left(\mathrm{CH}_{2} \mathrm{Cl}_{2}, 1 \% \mathrm{MeOH}\right)$.

${ }^{1} \mathbf{H}$ NMR (300 MHz, CDCl $)$ : syn-96: $\delta 0.83$ ppm (s, $\left.3 \mathrm{H}, 4-\mathrm{CH}_{3}\right), 1.41(\mathrm{dt}, J=14.3$, $\left.5.6 \mathrm{~Hz}, 1 \mathrm{H}, 5-\mathrm{H}_{\mathrm{a}}\right), 1.58-1.72\left(\mathrm{~m}, 3 \mathrm{H}, 3-\mathrm{H}_{2}, 5-\mathrm{H}_{\mathrm{b}}\right), 1.96$ (ddd, $J=16.3,9.2,6.4 \mathrm{~Hz}$, $1 \mathrm{H}, 2-\mathrm{H}_{\mathrm{a}}$ ), 2.10 (ddd, $J=16.3,9.0,7.6 \mathrm{~Hz}, 1 \mathrm{H}, 2-\mathrm{H}_{\mathrm{b}}$ ), 2.54 (m br., $\left.1 \mathrm{H}, \mathrm{OH}\right), 2.83$ $\left(\mathrm{dd}, J=13.3,5.5 \mathrm{~Hz}, 1 \mathrm{H}, 2^{\prime}-\mathrm{H}_{\mathrm{a}}\right), 2.90\left(\mathrm{dd}, J=13.3,7.9 \mathrm{~Hz}, 1 \mathrm{H}, 2^{\prime}-\mathrm{H}_{\mathrm{b}}\right), 3.51(\mathrm{~s}, 3 \mathrm{H}$, $\mathrm{CO}_{2} \mathrm{Me}$ ), 3.46-3.67 (2×m, $\left.2 \mathrm{H}, 6-\mathrm{H}_{2}\right), 4.60\left(\mathrm{dd}, J=7.9,5.5 \mathrm{~Hz}, 1 \mathrm{H}, 1^{\prime}-\mathrm{H}\right), 7.05-7.28$ $(2 \times \mathrm{m}, 10 \mathrm{H}, 2 \times \mathrm{Ph}) ; \underline{\text { anti-96}}$ (unterscheidbare Signale): 0.80 (s, 4- $\left.\mathrm{CH}_{3}\right), 3.62$ $\left(\mathrm{s}, \mathrm{CO}_{2} \mathrm{Me}\right)$.

${ }^{13} \mathbf{C}$ NMR (126 MHz, $\left.\mathrm{CDCl}_{3}\right)$ : $\underline{\text { syn-96}:} \delta 23.0$ ppm (4-CH $\left.\mathrm{CH}_{3}\right), 28.6(\mathrm{C}-2), 34.1(\mathrm{C}-3)$, 40.7 (C-5), $46.8\left(\mathrm{C}-2^{\prime}\right), 51.4\left(\mathrm{CO}_{2} \mathrm{CH}_{3}\right), 59.0(\mathrm{C}-6), 76.1\left(\mathrm{C}-1^{\prime}\right), 78.8(\mathrm{C}-4), 126.3$ (3×C-Ph), $127.1(\mathrm{C}-\mathrm{Ph}), 128.0(2 \times \mathrm{C}-\mathrm{Ph}), 128.2(2 \times \mathrm{C}-\mathrm{Ph}), 129.8(2 \times \mathrm{C}-\mathrm{Ph}), 138.3$ $\left(\mathrm{C}_{i}-\mathrm{Ph}\right), 144.5\left(\mathrm{C}_{i}-\mathrm{Ph}\right), 173.9(\mathrm{C}=\mathrm{O})$; anti-96 (unterscheidbare Signale): $24.0\left(4-\mathrm{CH}_{3}\right)$, 28.3 (C-2), 33.0 (C-3), 41.1 (C-5), $51.5\left(\mathrm{CO}_{2} \mathrm{CH}_{3}\right), 59.2$ (C-6), $76.1\left(\mathrm{C}-1^{\prime}\right), 78.9$ (C-4), $126.1(3 \times \mathrm{C}-\mathrm{Ph}), 128.2(2 \times \mathrm{C}-\mathrm{Ph}), 138.2\left(\mathrm{C}_{i}-\mathrm{Ph}\right), 144.8\left(\mathrm{C}_{i}-\mathrm{Ph}\right), 174.0(\mathrm{C}=\mathrm{O})$.

IR (ATR): $\tilde{v} 3269 \mathrm{~cm}^{-1}, 2926,1725(\mathrm{C}=\mathrm{O}), 1450,1432,1305,1284,1246,1143$, $1102,1047,1024,756,745$.

UV $\left(\mathrm{CH}_{3} \mathrm{CN}\right): \lambda_{\max }(\lg \varepsilon) 253 \mathrm{~nm}$ (2.6899), 259 (2.7345).

MS (ESI, MeOH): $m / z$ (\%) 357.2 (6) $[\mathrm{M}+\mathrm{H}]^{+}, 379.2(100)[\mathrm{M}+\mathrm{Na}]^{+}, 735.4$ (10) $[2 \mathrm{M}+\mathrm{Na}]^{+}$.

$\mathrm{C}_{22} \mathrm{H}_{28} \mathrm{O}_{4}$ (356.46)

ber.: 379.1880, gef.: $379.1879[\mathrm{M}+\mathrm{Na}]^{+}$(ESI-HRMS). 


\section{$5.3(+)-(R, R)-3,5-D i n i t r o b e n z o e s a ̈ u r e-[3-(1,2-d i p h e n y l e t h o x y)-$ 6-methoxy-3-methyl-6-oxohexyl]ester (97)}<smiles>CCCC(C)(C)OC(Cc1ccccc1)C(C)(CCOC(=O)c1cc([N+](=O)[O-])cc([N+](=O)[O-])c1)CCC(C)=O</smiles>

$\mathrm{Zu}$ einer Lösung des $(R, R)$-Alkohols $96 \mathbf{a}(1.2 \mathrm{~g}, 3.3 \mathrm{mmol}, d r$ : 95:5), 3,5-Dinitrobenzoylchlorid (1.0 g, $4.3 \mathrm{mmol}$ ) und DMAP ( $40 \mathrm{mg}, 0.33 \mathrm{mmol}, 10 \mathrm{~mol} \%$ ) in $\mathrm{CH}_{2} \mathrm{Cl}_{2}$ $(60 \mathrm{~mL})$ wurde bei $0{ }^{\circ} \mathrm{C} \mathrm{Et}_{3} \mathrm{~N}(0.81 \mathrm{~mL}, 5.7 \mathrm{mmol})$ getropft. Nach $2 \mathrm{~h}$ Rühren bei Umgebungstemperatur wurde die Reaktion durch Zugabe von gesättigter $\mathrm{NH}_{4} \mathrm{Cl}$-Lsg. $(10 \mathrm{~mL})$ beendet. Die wässrige Phase wurde mit $\mathrm{CH}_{2} \mathrm{Cl}_{2}(3 \times 20 \mathrm{~mL})$ extrahiert, die organischen Phasen vereinigt und Kieselgel $(7 \mathrm{~g})$ zugegeben. Entfernen des Lösungsmittels unter reduziertem Druck und säulenchromatographische Reinigung des Rückstandes an Kieselgel $\left(\mathrm{CHCl}_{3}, 1 \% \mathrm{EtOH}\right)$ liefert den Dinitrobenzoesäureester 97 (1.7 g, $3.0 \mathrm{mmol}, 91 \%, d r: 95: 5)$ als farblosen Feststoff.

Umkristallisation aus EtOAc/n-Heptan lieferte die epimerenreine Verbindung 97 (1.4 g, $2.5 \mathrm{mmol}, 82 \%, d r:>99: 1)$ als farblose, quaderförmige Kristalle.

R-Wert: $0.50\left(2 \times\left(\mathrm{CHCl}_{3}, 1 \% \mathrm{EtOH}\right)\right)$.

Schmelzpunkt: $136.5^{\circ} \mathrm{C}(d r:>99: 1)$.

Drehwert: $[\alpha]_{D}^{24}=+35.1^{\circ}\left(c 1, \mathrm{CHCl}_{3}\right)$.

${ }^{1} \mathbf{H}$ NMR $\left(300 \mathrm{MHz}, \mathrm{CDCl}_{3}\right): \delta 0.86 \mathrm{ppm}\left(\mathrm{s}, 3 \mathrm{H}, 3^{\prime}-\mathrm{CH}_{3}\right), 1.60-1.81\left(\mathrm{~m}, 4 \mathrm{H}, 2^{\prime}-\mathrm{H}_{2}\right.$, 4'- $\mathrm{H}_{2}$ ), 2.15 (ddd, $J=16.2,9.7,6.0 \mathrm{~Hz}, 1 \mathrm{H}, 5^{\prime}-\mathrm{H}_{\mathrm{a}}$ ), 2.25 (ddd, $J=16.2,10.1,6.3 \mathrm{~Hz}$, $\left.1 \mathrm{H}, 5^{\prime}-\mathrm{H}_{\mathrm{b}}\right), 2.84\left(\mathrm{dd}, J=13.5,5.0 \mathrm{~Hz}, 1 \mathrm{H}, 2^{\prime \prime}-\mathrm{H}_{\mathrm{a}}\right), 2.91(\mathrm{dd}, J=13.5,8.4 \mathrm{~Hz}, 1 \mathrm{H}$, 2"- $\mathrm{H}_{\mathrm{b}}$ ), 3.58 (s, $3 \mathrm{H}, \mathrm{CO}_{2} \mathrm{Me}$ ), 4.13 (ddd, $J=10.8,8.9,6.2 \mathrm{~Hz}, 1 \mathrm{H}, 1^{\prime}-\mathrm{H}_{\mathrm{a}}$ ), 4.26 (ddd, $\left.J=10.8,9.0,6.0 \mathrm{~Hz}, 1 \mathrm{H}, 1^{\prime}-\mathrm{H}_{\mathrm{b}}\right), 4.60\left(\mathrm{dd}, J=8.4,5.0 \mathrm{~Hz}, 1 \mathrm{H}, 1^{\prime \prime}-\mathrm{H}\right), 7.09-7.34$ (m, $10 \mathrm{H}, 2 \times \mathrm{Ph}), 9.06(\mathrm{~d}, J=2.2 \mathrm{~Hz}, 2 \mathrm{H}, 2-\mathrm{H}, 6-\mathrm{H}), 9.20$ (t, $J=2.2 \mathrm{~Hz}, 1 \mathrm{H}, 4-\mathrm{H})$. 
${ }^{13} \mathrm{C}$ NMR $\left(126 \mathrm{MHz}, \mathrm{CDCl}_{3}\right): \delta 23.6 \mathrm{ppm}\left(3^{\prime}-\mathrm{CH}_{3}\right), 28.5\left(\mathrm{C}-5^{\prime}\right), 34.5\left(\mathrm{C}-2^{\prime}\right), 36.9$ (C-4'), 46.8 (C-2"), $51.5\left(\mathrm{CO}_{2} \mathrm{CH}_{3}\right), 63.3\left(\mathrm{C}-1^{\prime}\right), 76.1,76.2\left(\mathrm{C}-3^{\prime}, \mathrm{C}-1^{\prime \prime}\right), 122.2(\mathrm{C}-4)$,

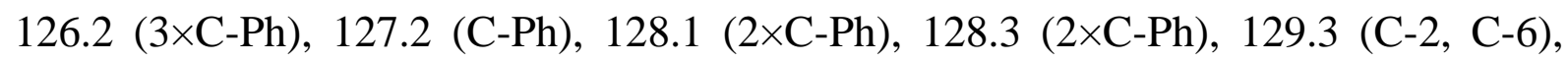
$129.8(2 \times \mathrm{C}-\mathrm{Ph}), 134.0(\mathrm{C}-1), 138.8\left(\mathrm{C}_{i}-\mathrm{Ph}\right), 145.0\left(\mathrm{C}_{i}-\mathrm{Ph}\right), 148.6(\mathrm{C}-3, \mathrm{C}-5), 162.3$ $\left(\mathrm{ArCO}_{2} \mathrm{CH}_{2}\right), 173.8\left(\mathrm{CO}_{2} \mathrm{CH}_{3}\right)$.

IR (ATR): $\tilde{v} 3102 \mathrm{~cm}^{-1}, 2923,1734(\mathrm{C}=\mathrm{O}), 1719(\mathrm{C}=\mathrm{O}), 1630,1546\left(\mathrm{NO}_{2}\right), 1454$, $1341\left(\mathrm{NO}_{2}\right), 1301,1281,1170,1148,1127,1072,1057,1017,980,965,940,918$, $911,857,826,768,753,740$.

UV $\left(\mathrm{CH}_{3} \mathrm{CN}\right): \lambda_{\max }(\lg \varepsilon) 209 \mathrm{~nm}(4.5529)$.

MS (ESI, MeOH): $m / z(\%) 573.2(100)[\mathrm{M}+\mathrm{Na}]^{+}, 1123.4(31)[2 \mathrm{M}+\mathrm{Na}]^{+}$.

$\mathbf{C}_{29} \mathbf{H}_{30} \mathbf{N}_{2} \mathbf{O}_{9}$ (550.56) ber.: 573.1844 , gef.: $573.1845[\mathrm{M}+\mathrm{Na}]^{+}$(ESI-HRMS).

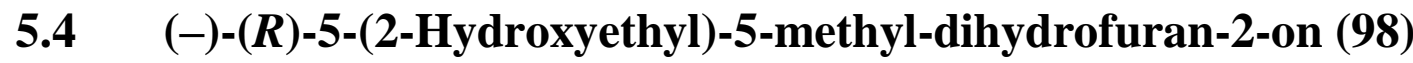

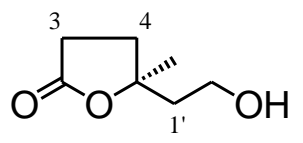

Analog zu AAV2 wurde der Alkohol $(R, R)$-96a (400 mg, $1.1 \mathrm{mmol}, d r:>99: 1)$ mit Wasserstoff (1 atm) in Gegenwart von $10 \mathrm{w} \%$ Palladium auf Aktivkohle (120 mg, $0.11 \mathrm{mmol}, 10 \mathrm{~mol} \%)$ in THF/MeOH 2:1 $(12 \mathrm{~mL})$ über $3 \mathrm{~h}$ umgesetzt. Nach Aufarbeitung und säulenchromatographischer Reinigung an Kieselgel $\left(\mathrm{CH}_{2} \mathrm{Cl}_{2}\right.$, $2 \% \mathrm{MeOH})$ wurde das $\gamma$-Lacton $98(160 \mathrm{mg}, 1.1 \mathrm{mmol}$, quant.) als farbloses Öl erhalten.

Drehwert: $[\alpha]_{D}^{23}=-3.7^{\circ}\left(c 1, \mathrm{CHCl}_{3}\right)$.

R-Wert: $0.10\left(\mathrm{CH}_{2} \mathrm{Cl}_{2}, 2 \% \mathrm{MeOH}\right)$.

${ }^{1} \mathbf{H}$ NMR $\left(300 \mathrm{MHz}, \mathrm{CDCl}_{3}\right): \delta 1.35 \mathrm{ppm}\left(\mathrm{s}, 3 \mathrm{H}, 5-\mathrm{CH}_{3}\right), 1.85(\mathrm{dt}, J=15.3,6.6 \mathrm{~Hz}$, $\left.1 \mathrm{H}, 1^{\prime}-\mathrm{H}_{\mathrm{a}}\right), 1.90\left(\mathrm{t}, J=15.3,6.5 \mathrm{~Hz}, 1 \mathrm{H}, 1^{\prime}-\mathrm{H}_{\mathrm{b}}\right), 1.95$ (ddd, $J=12.9,8.5,7.5 \mathrm{~Hz}, 1 \mathrm{H}$, 4- $\mathrm{H}_{\mathrm{a}}$ ), 2.16 (dt, $J=12.9,8.6 \mathrm{~Hz}, 1 \mathrm{H}, 4-\mathrm{H}_{\mathrm{b}}$ ), 2.50-2.57 (m, $2 \mathrm{H}, 3-\mathrm{H}_{2}$ ), 2.74 (s br., $1 \mathrm{H}$, $\mathrm{OH}), 3.66\left(\mathrm{dd}, J=11.0,6.5 \mathrm{~Hz}, 1 \mathrm{H}, 2^{\prime}-\mathrm{H}_{\mathrm{a}}\right), 3.73\left(\mathrm{dd}, J=11.0,6.6 \mathrm{~Hz}, 1 \mathrm{H}, 2^{\prime}-\mathrm{H}_{\mathrm{b}}\right)$. 
${ }^{13} \mathrm{C} \mathrm{NMR}\left(126 \mathrm{MHz}, \mathrm{CDCl}_{3}\right): \delta 25.7$ ppm (5-CH$), 28.8(\mathrm{C}-3), 33.4(\mathrm{C}-4), 42.6\left(\mathrm{C}-1^{\prime}\right)$, $58.0\left(\mathrm{C}-2^{\prime}\right), 86.1(\mathrm{C}-5), 176.9(\mathrm{C}=\mathrm{O})$.

IR (ATR): $\tilde{v} 3406 \mathrm{~cm}^{-1}$ (OH), 2972, 2935, 2891, 1749 (C=O), 1455, 1421, 1384, 1289, 1267, 1184, 1091, 1054, 1027, 933.

MS (ESI, MeOH): $m / z(\%) 167.1(11)[\mathrm{M}+\mathrm{Na}]^{+}, 311.2(100)[2 \mathrm{M}+\mathrm{Na}]^{+}$.

$\mathbf{C}_{7} \mathbf{H}_{12} \mathbf{O}_{3}(144.17) \quad$ ber.: 167.0679 , gef.: $167.0679[\mathrm{M}+\mathrm{Na}]^{+}(\mathrm{ESI}-\mathrm{HRMS})$.

\section{$5.5 \quad(-)-(R)-5-(2-H y d r o x y-4-m e t h y l-p e n t-3-e n y l)-5-m e t h y l-$ dihydrofuran-2-on (71)}

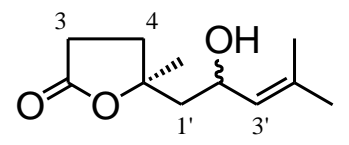

$\mathrm{Zu}$ einer Lösung des Lactons $98(10 \mathrm{mg}, 69 \mu \mathrm{mol})$ in $\mathrm{CH}_{2} \mathrm{Cl}_{2}(2 \mathrm{~mL})$ wurde DMP (60 mg, $140 \mu \mathrm{mol}$ ) bei $0{ }^{\circ} \mathrm{C}$ gegeben. Nach 20 min Rühren bei Umgebungstemperatur wurde die Lösung durch Kieselgel fraktioniert filtriert und mit $\mathrm{Et}_{2} \mathrm{O}(30 \mathrm{~mL})$ gewaschen. Die Fraktionen des gebildeten labilen Aldehyds wurden vereinigt und bei 600 mbar eingeengt. $\mathrm{Zu}$ einer Lösung des Rückstandes wurde bei $-60{ }^{\circ} \mathrm{C}$ in trockenem THF (2 mL) tropfenweise 2-Methyl-1-propenylmagnesiumbromid $(0.17 \mathrm{~mL}$ einer $0.5 \mathrm{M}$ Lsg. in THF, $85 \mu \mathrm{mol}$ ) gegeben. Nach $2 \mathrm{~h}$ Rühren bei $-60{ }^{\circ} \mathrm{C}$ wurde die Reaktion durch Zugabe von gesättigter $\mathrm{NH}_{4} \mathrm{Cl}$-Lsg. $(1 \mathrm{~mL})$ beendet. Die Lösung wurde mit MTBE $(3 \times 5 \mathrm{~mL})$ extrahiert, die vereinigten organischen Phasen mit gesättigter NaCl-Lsg. gewaschen und über $\mathrm{Na}_{2} \mathrm{SO}_{4}$ getrocknet. Entfernen des Lösungsmittels unter reduziertem Druck ( $>200 \mathrm{mbar}$ ) und anschließende säulenchromatographische Reinigung an Kieselgel (MTBE/Hexan 7:3) lieferte das Lacton $71(5.5 \mathrm{mg}, 28 \mu \mathrm{mol}, 40 \%)$ mit einer Diastereoselektivität von 1:1.2 als farbloses Öl.

Drehwert: $[\alpha]_{D}^{23}=-13.5^{\circ}\left(c 0.41, \mathrm{CHCl}_{3}\right)$.

R-Wert: 0.42 (MTBE), 0.18 (MTBE/Hexan 7:3), $0.16\left(\mathrm{CH}_{2} \mathrm{Cl}_{2}, 2 \% \mathrm{MeOH}\right)$. 
${ }^{1} \mathbf{H}$ NMR (300 MHz, $\left.\mathrm{CDCl}_{3}\right): \delta 1.42 \mathrm{ppm}, 1.44\left(2 \times \mathrm{s}, 6 \mathrm{H}, 2 \times 5-\mathrm{CH}_{3}\right), 1.66,1.68(2 \times \mathrm{s}$, $\left.12 \mathrm{H}, 2 \times 5^{\prime} \mathrm{a}-\mathrm{H}_{3}, 2 \times 5^{\prime} \mathrm{b}-\mathrm{H}_{3}\right), 1.74\left(\mathrm{dd}, J=14.6,3.7 \mathrm{~Hz}, 2 \mathrm{H}, 2 \times 1^{\prime}-\mathrm{H}_{\mathrm{a}}\right), 1.88-2.04$ $\left(\mathrm{m}, 4 \mathrm{H}, 2 \times 1^{\prime}-\mathrm{H}_{\mathrm{b}}, 2 \times 4-\mathrm{H}_{\mathrm{a}}\right), 2.27\left(\mathrm{dt}, J=12.9,8.7 \mathrm{~Hz}, 2 \mathrm{H}, 2 \times 4-\mathrm{H}_{\mathrm{b}}\right), 2.53-2.62(\mathrm{~m}, 4 \mathrm{H}$, $\left.2 \times 3-\mathrm{H}_{2}\right), 4.51-4.64\left(\mathrm{~m}, 2 \mathrm{H}, 2 \times 2^{\prime}-\mathrm{H}\right), 5.18\left(\mathrm{~d}, J=8.7 \mathrm{~Hz}, 2 \mathrm{H}, 2 \times 3^{\prime}-\mathrm{H}\right)$.

${ }^{13} \mathbf{C}$ NMR $\left(126 \mathrm{MHz}, \mathrm{CDCl}_{3}\right): \delta 18.1 \mathrm{ppm}\left(2 \times \mathrm{C}-5{ }^{\prime} \mathrm{a}\right), 25.6,26.0,26.5,26.9(2 \times \mathrm{C}-5$ 'b, $\left.2 \times 5-\mathrm{CH}_{3}\right), 28.7,29.0(2 \times \mathrm{C}-3), 33.6,33.8(2 \times \mathrm{C}-4), 47.3,47.7\left(2 \times \mathrm{C}-1^{\prime}\right), 65.0,65.4$ $\left(2 \times \mathrm{C}-2^{\prime}\right), 85.9,86.3(2 \times \mathrm{C}-5), 127.7,127.9\left(2 \times \mathrm{C}-3^{\prime}\right), 134.9,135.0\left(2 \times \mathrm{C}-4^{\prime}\right), 176.6$, $176.7(2 \times \mathrm{C}=\mathrm{O})$.

IR (ATR): $\tilde{v} 3430 \mathrm{~cm}^{-1}(\mathrm{OH}), 2972,2931,1753(\mathrm{C}=\mathrm{O}), 1672,1449,1380,1175,1080$, 933.

MS (ESI, MeOH): m/z (\%) $221.1(35)[\mathrm{M}+\mathrm{Na}]^{+}, 419.3$ (100) $[2 \mathrm{M}+\mathrm{Na}]^{+} ; 197.1$ (2) $[\mathrm{M}-\mathrm{H}]^{-}, 243.1(100)\left[\mathrm{M}+\mathrm{HCO}_{2}\right]^{-}$.

$\mathbf{C}_{11} \mathbf{H}_{18} \mathbf{O}_{3}$ (198.26) ber.: 221.1148, gef.: $221.1149[\mathrm{M}+\mathrm{Na}]^{+}$(ESI-HRMS).

\section{$5.6 \quad(+)-(R)$-Pinnatolid (25)}

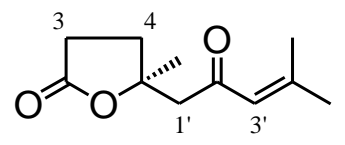

A: $\mathrm{Zu}$ einer Lösung des Alkohols $(R)-71(2.0 \mathrm{mg}, 10 \mu \mathrm{mol})$ in $\mathrm{CH}_{2} \mathrm{Cl}_{2}(0.5 \mathrm{~mL})$ wurde bei $0{ }^{\circ} \mathrm{C}$ DMP $(8.5 \mathrm{mg}, 20 \mu \mathrm{mol})$ gegeben und $2 \mathrm{~h}$ bei Umgebungstemperatur gerührt. Die Suspension wurde über Kieselgel filtriert, mit $\mathrm{Et}_{2} \mathrm{O}(2 \times 10 \mathrm{~mL})$ gewaschen und das Lösungmittel unter reduziertem Druck ( $p \geq 500$ mbar) entfernt. Nach säulenchromatographischer Reinigung des Rückstandes an Kieselgel ( $\mathrm{P} / \mathrm{Et}_{2} \mathrm{O}$ 5:3) wurde das Enon (R)-25 (1.8 mg, $10 \mu \mathrm{mol}, 90 \%)$ als farbloses Öl erhalten.

B: $\mathrm{Zu}$ einer Lösung des $\gamma$-Lactons $(R)-82(100 \mathrm{mg}, 0.55 \mathrm{mmol})$ in EtOAc $(2.0 \mathrm{~mL})$ wurde bei Umgebungstemperatur tert-Butylhydroperoxid $(0.50 \mathrm{~mL}$ einer $5.5 \mathrm{M} \mathrm{Lsg}$. in n-Dekan, $2.75 \mathrm{mmol}), 4 \AA$ Molekularsieb (100 mg) und Mangan(III)-acetat-dihydrat (30.0 mg, $0.11 \mathrm{mmol}, 20 \mathrm{~mol} \%)$ gegeben und $36 \mathrm{~h}$ gerührt. Anschließend wurde die Suspension über Kieselgur filtriert, mit EtOAc $(2 \times 5 \mathrm{~mL})$ gewaschen und das Lösungsmittel unter reduziertem Druck entfernt. Säulenchromatographische Reinigung des 
Rückstands an Kieselgel $\left(\mathrm{CH}_{2} \mathrm{Cl}_{2} \rightarrow \mathrm{CH}_{2} \mathrm{Cl}_{2} /\right.$ Aceton 25:1) lieferte das Enon (S)-25 (33 mg, $0.17 \mathrm{mmol}, 31 \%$ ) als farbloses Öl.

Drehwert: $[\alpha]_{D}^{23}=+10.8^{\circ}\left(c 0.21, \mathrm{CHCl}_{3},(R)-25\right)$.

$\mathbf{R}_{\boldsymbol{f}}$-Wert: 0.46 (MTBE).

HPLC (Chiralpak ${ }^{\circledR}$ IB, $n$-Hexan/i-PrOH 85:15, $0.6 \mathrm{~mL} / \mathrm{min}, 237 \mathrm{~nm}, 1 \mathrm{mg} / \mathrm{mL}, 3 \mu \mathrm{L}$ ): $t_{\mathrm{R}}=14.2 \min (S), 14.7(R)$.

${ }^{1} \mathbf{H}$ NMR (300 MHz, $\left.\mathrm{CDCl}_{3}\right): \delta 1.45$ ppm (s, $\left.3 \mathrm{H}, 5-\mathrm{CH}_{3}\right), 1.87\left(\mathrm{~m}_{\mathrm{c}}, 3 \mathrm{H}, 5^{\prime} \mathrm{a}-\mathrm{H}_{3}\right), 2.12$ $\left(\mathrm{m}_{\mathrm{c}}, 3 \mathrm{H}, 5^{\prime} \mathrm{b}-\mathrm{H}_{3}\right), 2.11\left(\mathrm{ddd}, J=13.1,8.4,7.4 \mathrm{~Hz}, 1 \mathrm{H}, 4-\mathrm{H}_{\mathrm{a}}\right), 2.32(\mathrm{ddd}, J=13.1,9.4$, $\left.8.3 \mathrm{~Hz}, 1 \mathrm{H}, 4-\mathrm{H}_{\mathrm{b}}\right), 2.59\left(\mathrm{~m}_{\mathrm{c}}, 2 \mathrm{H}, 3-\mathrm{H}_{2}\right), 2.78$ (d, J=16.1 Hz, $\left.1 \mathrm{H}, 1^{\prime}-\mathrm{H}_{\mathrm{a}}\right), 2.83$ (d, $\left.J=16.1 \mathrm{~Hz}, 1 \mathrm{H}, 1^{\prime}-\mathrm{H}_{\mathrm{b}}\right), 6.06$ (sep, $\left.J=1.3 \mathrm{~Hz}, 1 \mathrm{H}, 3^{\prime}-\mathrm{H}\right)$.

${ }^{13} \mathrm{C} \mathrm{NMR}\left(126 \mathrm{MHz}, \mathrm{CDCl}_{3}\right): \delta 21.0$ ppm (C-5'b), $26.7\left(5-\mathrm{CH}_{3}\right), 27.9(\mathrm{C}-5$ 'a), 28.9 (C-3), 32.6 (C-4), 53.5 (C-1'), 84.8 (C-5), 124.0 (C-3'), 157.2 (C-4'), 176.4 (C-2), $196.5\left(\mathrm{C}-2^{\prime}\right)$.

IR (ATR): $\tilde{v} 2931 \mathrm{~cm}^{-1}, 1767(\mathrm{C}=\mathrm{O}), 1686,1617,1444,1378,1278,1174,1102$, 1077, 1044, 942.

UV $\left(\mathrm{CH}_{3} \mathrm{CN}\right): \lambda_{\max }(\lg \varepsilon) 237 \mathrm{~nm}(3.2705)$.

MS (ESI, MeOH): $m / z(\%) 219.1(100)[\mathrm{M}+\mathrm{Na}]^{+}, 415.2(90)[2 \mathrm{M}+\mathrm{Na}]^{+}$.

$\mathbf{C}_{11} \mathbf{H}_{16} \mathbf{O}_{3}(196.24)$ ber.: 219.0992, gef.: 219.0992 [M+Na $]^{+}$(ESI-HRMS). 


\section{Synthese von Furopinnatin}

\section{1 $\quad r a c-(R, R)-5-(1,2-D i p h e n y l e t h o x y)-5-m e t h y l o x e p a n-2-o n(102)$}

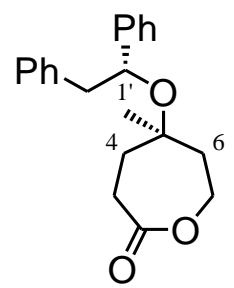

$\mathrm{Zu}$ einer Lösung des Alkohols rac-96a (200 mg, $560 \mu \mathrm{mol})$ in $\mathrm{CHCl}_{3}(20 \mathrm{~mL})$ wurde bei Umgebungstemperatur $p$-Toluolsulfonsäure-Monohydrat $\quad(5.6 \mathrm{mg}, 29 \mu \mathrm{mol}$, $5 \mathrm{~mol} \%$ ) gegeben und $3 \mathrm{~h}$ bei $60{ }^{\circ} \mathrm{C}$ gerührt. Anschließend wurde die Lösung mit halbgesättigter $\mathrm{NaHCO}_{3}$-Lsg. (20 mL) gewaschen, die wässrige Phase mit $\mathrm{CH}_{2} \mathrm{Cl}_{2}$ $(2 \times 20 \mathrm{~mL})$ extrahiert, die vereinigten organischen Phasen über $\mathrm{Na}_{2} \mathrm{SO}_{4}$ getrocknet und das Lösungsmittel unter reduziertem Druck entfernt. Nach säulenchromatographischer Reinigung an Kieselgel (PE/MTBE 6:4) wurde das $\varepsilon$-Lacton (178 mg, $0.55 \mathrm{mmol}$, 97\%) als farbloses Öl erhalten.

R -Wert: 0.42 (PE/MTBE 1:1).

${ }^{1} \mathbf{H}$ NMR $\left(300 \mathrm{MHz}, \quad \mathrm{CDCl}_{3}\right) ： \underline{s y n-102}: \delta \quad \delta .80 \mathrm{ppm} \quad\left(\mathrm{s}, \quad 3 \mathrm{H}, \quad 5-\mathrm{CH}_{3}\right), 1.44 \quad(\mathrm{t}$, $\left.J=13.3 \mathrm{~Hz}, 1 \mathrm{H}, 4-\mathrm{H}_{\mathrm{a}}\right), 1.51\left(\mathrm{ddd}, J=15.8,10.7,1.9 \mathrm{~Hz}, 1 \mathrm{H}, 6-\mathrm{H}_{\mathrm{a}}\right), 1.77-1.89$ (m, $\left.2 \mathrm{H}, 4-\mathrm{H}_{\mathrm{b}}, 6-\mathrm{H}_{\mathrm{b}}\right), 2.01\left(\mathrm{ddd}, J=14.2,7.3,1.2 \mathrm{~Hz}, 1 \mathrm{H}, 3-\mathrm{H}_{\mathrm{a}}\right), 2.70(\mathrm{t}, J=13.4 \mathrm{~Hz}$, $\left.1 \mathrm{H}, 3-\mathrm{H}_{\mathrm{b}}\right), 2.88\left(\mathrm{dd}, J=13.6,5.3 \mathrm{~Hz}, 1 \mathrm{H}, 2^{\prime}-\mathrm{H}_{\mathrm{a}}\right), 2.93(\mathrm{dd}, J=13.6,8.3 \mathrm{~Hz}, 1 \mathrm{H}$, 2'- $\mathrm{H}_{\mathrm{b}}$ ), 3.61 (ddd, $J=13.1,5.2,1.8 \mathrm{~Hz}, 1 \mathrm{H}, 7-\mathrm{H}_{\mathrm{a}}$ ), 3.83 (dd, $J=13.1,10.7 \mathrm{~Hz}, 7-\mathrm{H}_{\mathrm{b}}$ ), 4.57 (dd, $\left.J=8.3,5.3 \mathrm{~Hz}, 1^{\prime}-\mathrm{H}\right), 7.14-7.36(\mathrm{~m}, 10 \mathrm{H}, 2 \times \mathrm{Ph}$ ); anti-102 (unterscheidbare Signale): $2.37\left(\mathrm{t}, J=13.4 \mathrm{~Hz}, 3-\mathrm{H}_{\mathrm{b}}\right), 3.69\left(\mathrm{ddd}, J=13.1,5.2,1.8 \mathrm{~Hz}, 7-\mathrm{H}_{\mathrm{a}}\right), 4.18$ (dd, $J=13.0,10.8 \mathrm{~Hz}, 7-\mathrm{H}_{\mathrm{b}}$ ).

${ }^{13} \mathbf{C}$ NMR (126 MHz, $\left.\mathrm{CDCl}_{3}\right)$ : syn-102: $\delta 26.9$ ppm (5- $\left.\mathrm{CH}_{3}\right), 28.2(\mathrm{C}-3), 34.5(\mathrm{C}-4)$, 39.2 (C-6), 46.7 (C-2'), 62.9 (C-7), 74.5 (C-5), 76.1 (C-1'), 126.0 (2×C-Ph), 126.4 (C-Ph), $127.3(\mathrm{C}-\mathrm{Ph}), 128.2(2 \times \mathrm{C}-\mathrm{Ph}), 128.3(2 \times \mathrm{C}-\mathrm{Ph}), 129.6(2 \times \mathrm{C}-\mathrm{Ph}), 138.8$ $\left(\mathrm{C}_{i}-\mathrm{Ph}\right), 145.0\left(\mathrm{C}_{i}-\mathrm{Ph}\right), 176.3(\mathrm{C}=\mathrm{O})$; anti-102 (unterscheidbare Signale): $27.1\left(5-\mathrm{CH}_{3}\right)$, 27.4 (C-3), 32.8 (C-4), 40.9 (C-6), 46.8 (C-2'), 63.4 (C-7), 72.7 (C-5), 76.1 (C-1'), $126.1(2 \times \mathrm{C}-\mathrm{Ph}), 126.5(\mathrm{C}-\mathrm{Ph}), 129.6(2 \times \mathrm{C}-\mathrm{Ph}), 138.8\left(\mathrm{C}_{i}-\mathrm{Ph}\right), 145.1\left(\mathrm{C}_{i}-\mathrm{Ph}\right)$. 
IR (ATR): $\tilde{v} 3061 \mathrm{~cm}^{-1}, 3027,2975,2928,1737,1602,1495,1453,1386,1332,1291$, 1255, 1173, 1131, 1102, 1074, 1057, 1020, 945, 760, 698.

UV $\left(\mathrm{CH}_{3} \mathrm{CN}\right): \lambda_{\max }(\lg \varepsilon) 254 \mathrm{~nm}(2.5414), 259$ (2.6154).

MS (ESI, MeOH): $m / z(\%) 347.2$ (45) $[\mathrm{M}+\mathrm{Na}]^{+}, 671.4$ (100) [2M+Na] ${ }^{+} ; 359.1$ (17)

$[\mathrm{M}+\mathrm{Cl}]^{-}, 369.2(100)\left[\mathrm{M}+\mathrm{HCO}_{2}\right]^{-}$.

$\mathbf{C}_{21} \mathbf{H}_{24} \mathbf{O}_{3}(324.41) \quad$ ber.: 347.1618 ,

gef.: $347.1618[\mathrm{M}+\mathrm{Na}]^{+}$(ESI-HRMS).

\section{2 rac-(R,R)-4-(1,2-Diphenylethoxy)-1-(3-furyl)-6-hydroxy-4-methyl- 1-hexanon (104)}<smiles>C[C@H](CCC(=O)c1ccoc1)[C@]1(CCO)O[C@H](c2ccccc2)O1</smiles>

$\mathrm{Zu}$ einer Lösung von 3-Bromfuran $(19 \mu \mathrm{L}, 210 \mu \mathrm{mol})$ in THF (1 mL) wurde bei $-78^{\circ} \mathrm{C}$ langsam $n$-BuLi $(80 \mu \mathrm{L}$ einer $2.5 \mathrm{M}$ Lsg. in Hexan, $200 \mu \mathrm{mol})$ gegeben und 15 min bei dieser Temperatur gerührt. Nach Abkühlen der Lösung auf $-100{ }^{\circ} \mathrm{C}$ $\left(\mathrm{EtOH} / \mathrm{N}_{2(1)}\right)$ wurde langsam eine $0.2 \mathrm{M}$ Lösung des $\varepsilon$-Lacton 102 (64 mg, $\left.200 \mu \mathrm{mol}\right)$ in THF zugetropft. Nun wurde die Lösung über $2 \mathrm{~h}$ langsam auf $-60{ }^{\circ} \mathrm{C}$ erwärmt und dann die Reaktion durch Zugabe von gesättigter $\mathrm{NH}_{4} \mathrm{Cl}$-Lsg. (1 mL) beendet. Die wässrige Phase wurde mit MTBE $(2 \times 3 \mathrm{~mL})$ extrahiert, die vereinigten organischen Phasen mit gesättigter NaCl-Lsg. gewaschen, über $\mathrm{Na}_{2} \mathrm{SO}_{4}$ getrocknet und das Lösungsmittel unter reduziertem Druck entfernt. Säulenchromatographische Reinigung an Kieselgel (PE/MTBE 1:1) lieferte das 3-Acylfuran 104 (36 mg, $92 \mu \mathrm{mol}$, $46 \%$ ) als farblosen Feststoff.

Rf-Wert: 0.16 (PE/MTBE 1:1).

${ }^{1} \mathbf{H}$ NMR $\left(300 \mathrm{MHz}, \mathrm{CDCl}_{3}\right): \delta 0.94 \mathrm{ppm}\left(\mathrm{s}, 3 \mathrm{H}, 4-\mathrm{CH}_{3}\right), 1.45(\mathrm{dt}, J=14.4,5.2 \mathrm{~Hz}$, $\left.1 \mathrm{H}, 5-\mathrm{H}_{\mathrm{a}}\right), 1.71-1.86\left(\mathrm{~m}, 3 \mathrm{H}, 3-\mathrm{H}_{2}, 5-\mathrm{H}_{\mathrm{b}}\right), 2.31(\mathrm{ddd}, J=17.0,8.9,6.2 \mathrm{~Hz}, 1 \mathrm{H}$, 2- $\mathrm{H}_{\mathrm{a}}$ ), 2.48 (ddd, $J=17.0,8.7,6.9 \mathrm{~Hz}, 1 \mathrm{H}, 2-\mathrm{H}_{\mathrm{b}}$ ), 2.75 (s br., $\left.1 \mathrm{H}, \mathrm{OH}\right), 2.88$ (dd, $\left.J=13.5,5.6 \mathrm{~Hz}, 1 \mathrm{H}, 2^{\prime \prime}-\mathrm{H}_{\mathrm{a}}\right), 2.97\left(\mathrm{dd}, J=13.5,7.9 \mathrm{~Hz}, 1 \mathrm{H}, 2^{\prime \prime}-\mathrm{H}_{\mathrm{b}}\right), 3.59\left(\mathrm{~m}_{\mathrm{c}}, 1 \mathrm{H}\right.$, 
6- $\left.\mathrm{H}_{\mathrm{a}}\right), 3.74\left(\mathrm{~m}_{\mathrm{c}}, 1 \mathrm{H}, 6-\mathrm{H}_{\mathrm{b}}\right), 4.65\left(\mathrm{dd}, J=7.9,5.6 \mathrm{~Hz}, 1 \mathrm{H}, 1^{\prime \prime}-\mathrm{H}\right), 6.58(\mathrm{dd}, J=1.9$, $\left.0.8 \mathrm{~Hz}, 1 \mathrm{H}, 4^{\prime}-\mathrm{H}\right), 7.09$ (d, $\left.J=7.8 \mathrm{~Hz}, 2 \mathrm{H}, 2 \times \mathrm{Ph}-\mathrm{H}\right), 7.14-7.30(\mathrm{~m}, 8 \mathrm{H}, 8 \times \mathrm{Ph}-\mathrm{H})$, 7.33 (t, $\left.J=1.6 \mathrm{~Hz}, 1 \mathrm{H}, 5^{\prime}-\mathrm{H}\right), 7.58\left(\mathrm{dd}, J=1.4,0.8 \mathrm{~Hz}, 1 \mathrm{H}, 2^{\prime}-\mathrm{H}\right)$.

${ }^{13} \mathrm{C}$ NMR (126 MHz, $\mathrm{CDCl}_{3}$ ): $\delta 23.2 \mathrm{ppm}\left(4-\mathrm{CH}_{3}\right), 32.6$ (C-3), 34.6 (C-2), 40.9 (C-5), 46.7 (C-2"), 59.2 (C-6), 76.0 (C-1"), 79.2 (C-4), 108.4 (C-4'), 126.3 (C-Ph), 126.4

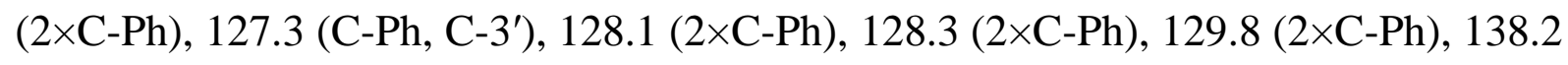
$\left(\mathrm{C}_{i}-\mathrm{Ph}\right), 143.8\left(\mathrm{C}-5^{\prime}\right), 144.4\left(\mathrm{C}_{i}-\mathrm{Ph}\right), 146.9\left(\mathrm{C}-2^{\prime}\right), 194.5(\mathrm{C}=\mathrm{O})$.

IR (ATR): $\tilde{v} 3559 \mathrm{~cm}^{-1}, 3124,2948,2915,1671(\mathrm{C}=\mathrm{O}), 1561,1509,1452,1153$, 1056, 1012, 990, 871, 831, 759, 700.

UV $\left(\mathrm{CH}_{3} \mathrm{CN}\right): \lambda_{\max }(\lg \varepsilon) 252 \mathrm{~nm}(4.5117)$.

MS (ESI, MeOH): $m / z(\%) 415.2(100)[\mathrm{M}+\mathrm{Na}]^{+}, 807.4(89)[2 \mathrm{M}+\mathrm{Na}]^{+} ; 427.2(11)$

$[\mathrm{M}+\mathrm{Cl}]^{-}, 437.2(100)\left[\mathrm{M}+\mathrm{HCO}_{2}\right]^{-}$.

$\mathbf{C}_{25} \mathbf{H}_{28} \mathbf{O}_{\mathbf{4}}$ (392.49) $\quad$ ber.: 415.1880 , gef.: $415.1879[\mathrm{M}+\mathrm{Na}]^{+}$(ESI-HRMS).

\section{3 rac-(4R,1"R)-4-(1,2-Diphenylethoxy)-1-(tetrahydrofur-3-yl)- 6-hydroxy-4-methyl-1-hexanon (105)}

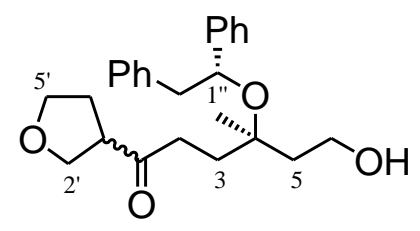

Analog zu AAV2 wurde das Acylfurans $104(19 \mathrm{mg}, 48 \mu \mathrm{mol})$ mit Wasserstoff (1 atm) in Gegenwart von $10 \mathrm{w} \%$ Palladium auf Aktivkohle $(5.2 \mathrm{mg}, 4.8 \mu \mathrm{mol}$, $10 \mathrm{~mol} \%)$ in THF/MeOH 2:1 (3 mL) über $2 \mathrm{~h}$ umgesetzt. Nach Aufarbeitung und säulenchromatographischer Reinigung an Kieselgel (MTBE/PE 9:1) wurde das Tetrahydrofuran 105 (13.5 mg, $34 \mu \mathrm{mol}, 71 \%)$ als ein Diasteoreomerengemisch (1:1) in Form eines weißen Feststoffs erhalten. 


\section{R -Wert: 0.19 (MTBE).}

${ }^{1} \mathbf{H}$ NMR $\left(300 \mathrm{MHz}, \mathrm{CDCl}_{3}\right): \delta 0.89 \mathrm{ppm}\left(\mathrm{s}, 6 \mathrm{H}, 2 \times 4-\mathrm{CH}_{3}\right), 1.36-1.41(\mathrm{~m}, 2 \mathrm{H}$, $\left.2 \times 5-\mathrm{H}_{\mathrm{a}}\right), 1.42-1.52\left(\mathrm{~m}, 2 \mathrm{H}, 2 \times 3-\mathrm{H}_{\mathrm{a}}\right), 1.65-1.72\left(\mathrm{~m}, 2 \mathrm{H}, 2 \times 3-\mathrm{H}_{\mathrm{b}}\right), 1.72-1.82(\mathrm{~m}, 4 \mathrm{H}$, $\left.4^{\prime}-\mathrm{H}_{2}(\mathrm{I}), 2 \times 5-\mathrm{H}_{\mathrm{b}}\right), 1.82-1.91\left(\mathrm{~m}, 2 \mathrm{H}, 4^{\prime}-\mathrm{H}_{2}(\mathrm{II})\right), 1.99$ (ddd, $J=17.9,10.7,4.4 \mathrm{~Hz}, 1 \mathrm{H}$, 2- $\left.\mathrm{H}_{\mathrm{a}}(\mathrm{I})\right), 2.04\left(\mathrm{ddd}, J=17.8,10.6,4.4 \mathrm{~Hz}, 1 \mathrm{H}, 2-\mathrm{H}_{\mathrm{a}}(\mathrm{II})\right), 2.17$ (ddd, $J=17.8,10.7$, $\left.5.5 \mathrm{~Hz}, 1 \mathrm{H}, 2-\mathrm{H}_{\mathrm{b}}(\mathrm{II})\right), 2.21\left(\mathrm{ddd}, J=17.9,10.8,5.5 \mathrm{~Hz}, 1 \mathrm{H}, 2-\mathrm{H}_{\mathrm{b}}(\mathrm{I})\right), 2.76\left(\mathrm{~m}_{\mathrm{c}}, 2 \mathrm{H}\right.$, $\left.2 \times 3^{\prime}-\mathrm{H}\right), 2.86\left(\mathrm{dd}, J=13.5,5.5 \mathrm{~Hz}, 2 \mathrm{H}, 2 \times 2^{\prime \prime}-\mathrm{H}_{\mathrm{a}}\right), 2.93(\mathrm{dd}, J=13.5,8.1 \mathrm{~Hz}, 2 \mathrm{H}$, $\left.2 \times 2^{\prime \prime}-\mathrm{H}_{\mathrm{b}}\right), 3.47\left(\mathrm{dd}, J=8.7,6.5 \mathrm{~Hz}, 1 \mathrm{H}, 2^{\prime}-\mathrm{H}_{\mathrm{a}}(\mathrm{I})\right), 3.57(\mathrm{dt}, J=11.1,5.4 \mathrm{~Hz}, 2 \mathrm{H}$, $\left.2 \times 6-\mathrm{H}_{\mathrm{a}}\right), 3.60-3.67\left(\mathrm{~m}, 2 \mathrm{H}, 2^{\prime}-\mathrm{H}_{\mathrm{b}}(\mathrm{I}), 2^{\prime}-\mathrm{H}_{\mathrm{a}}(\mathrm{II})\right), 3.66-3.76\left(\mathrm{~m}, 7 \mathrm{H}, 2 \times 5^{\prime}-\mathrm{H}_{2}, 2 \times 6-\mathrm{H}_{\mathrm{b}}\right.$, $\left.2^{\prime}-\mathrm{H}_{\mathrm{b}}(\mathrm{II})\right), 4.58-4.61\left(\mathrm{~m}, 2 \mathrm{H}, 2 \times 1^{\prime \prime}-\mathrm{H}\right), 7.07-7.09,7.17-7.29(2 \times \mathrm{m}, 20 \mathrm{H}, 2 \times(2 \times \mathrm{Ph}))$.

${ }^{13}$ C NMR (126 MHz, $\left.\mathrm{CDCl}_{3}\right): \delta 23.3\left(2 \times 4-\mathrm{CH}_{3}\right), 28.8,29.0\left(2 \times \mathrm{C}-4{ }^{\prime}\right), 32.4(2 \times \mathrm{C}-3)$, $36.5,36.6(2 \times \mathrm{C}-2), 41.0(2 \times \mathrm{C}-5), 46.8\left(2 \times \mathrm{C}-2^{\prime \prime}\right), 50.7\left(2 \times \mathrm{C}-3^{\prime}\right), 59.2(2 \times \mathrm{C}-6), 68.2$ $\left(2 \times \mathrm{C}-5^{\prime}\right), 69.3\left(2 \times \mathrm{C}-2^{\prime}\right), 76.0\left(2 \times \mathrm{C}-1^{\prime \prime}\right), 79.1 \quad(2 \times \mathrm{C}-4), 126.3(2 \times(\mathrm{C}-\mathrm{Ph})), 126.4$ $(2 \times(2 \times \mathrm{C}-\mathrm{Ph})), 127.2,127.3(2 \times(\mathrm{C}-\mathrm{Ph})), 128.0(2 \times(2 \times \mathrm{C}-\mathrm{Ph})), 128.2(2 \times(2 \times \mathrm{C}-\mathrm{Ph}))$, $129.7(2 \times(2 \times \mathrm{C}-\mathrm{Ph})), 138.1\left(2 \times\left(\mathrm{C}_{i}-\mathrm{Ph}\right)\right), 144.3\left(2 \times\left(\mathrm{C}_{i}-\mathrm{Ph}\right)\right), 209.1,209.2(2 \times \mathrm{C}=\mathrm{O})$.

IR (ATR): $\tilde{v} 3537 \mathrm{~cm}^{-1}(\mathrm{OH}), 3061,3029,2932,2874,1707(\mathrm{C}=\mathrm{O}), 1603,1495$, 1452, 1411, 1383, 1116, 1044, 1013, 908, 763, 732, 700.

UV ( $\left.\mathrm{CH}_{3} \mathrm{CN}\right): \lambda_{\max }(\lg \varepsilon) 254 \mathrm{~nm}$ (2.5103), 259 (2.5826).

MS (ESI, MeOH): m/z (\%) $419.2(55)[\mathrm{M}+\mathrm{Na}]^{+}, 815.4(100)[2 \mathrm{M}+\mathrm{Na}]^{+} ; 395.2(22)$ $[\mathrm{M}-\mathrm{H}]^{-}, 431.2(7)[\mathrm{M}+\mathrm{Cl}]^{-}, 441.2(100)\left[\mathrm{M}+\mathrm{HCO}_{2}\right]^{-}$.

\section{$\mathbf{C}_{25} \mathbf{H}_{32} \mathbf{O}_{4}(396.52)$}

ber.: 419.2193, gef.: $419.2192[\mathrm{M}+\mathrm{Na}]^{+}$(ESI-HRMS); ber.: 395.2228, gef.: 395.2215 [M-H] $]^{-}$(ESI-HRMS). 


\section{4 (+)-(5R,1"R)-5-(1,2-Diphenylethoxy)-5-methyl-7-(2-methyl- prop-1-enyl)oxepan-2-on (111a+b)}

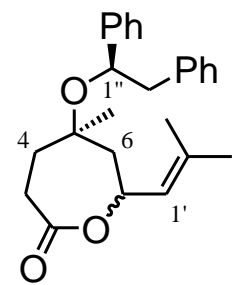

A: $\mathrm{Zu}$ einer auf $-60{ }^{\circ} \mathrm{C}$ gekühlten Lösung des Aldehyds 95a $(80 \mathrm{mg}, 0.23 \mathrm{mmol})$ in THF (4.5 mL) wurde über die vorgekühlte Gefäßwand langsam 2-Methyl-1-propenylmagnesiumbromid $(0.55 \mathrm{~mL}$ einer $0.5 \mathrm{M} \mathrm{Lsg}$. in THF, $0.27 \mathrm{mmol}$ ) zugetropft (ca. $20 \mathrm{~min})$. Der an der Gefäßwand ausgefallene Grignard wurde durch Nachspülen mit THF (0.1 mL) abgelöst und die gebildete Lösung $1 \mathrm{~h}$ bei dieser Temperatur gerührt. Anschließend wurde die Reaktion durch Zugabe von ges. $\mathrm{NH}_{4} \mathrm{Cl}$-Lsg. (1 mL) beendet, die wässrige Phase mit MTBE $(3 \times 10 \mathrm{~mL})$ extrahiert, die vereinigten organischen Phasen mit ges. NaCl-Lsg. ( $5 \mathrm{~mL}$ ) gewaschen und über $\mathrm{Na}_{2} \mathrm{SO}_{4}$ getrocknet. Säulenchromatographische Reinigung an Kieselgel $\left(\mathrm{CHCl}_{3}, 0.5 \% \mathrm{EtOH} ; \mathrm{PE} / \mathrm{MTBE}\right.$ 5:1, $0.3 \% i$-PrOH) lieferte die $\varepsilon$-Lactone 111a+b (65 mg, $0.17 \mathrm{mmol}, 76 \%, d r: 1: 1.3$ ) jeweils als farblose Öle.

B: $\mathrm{Zu}$ einer auf $-60^{\circ} \mathrm{C}$ gekühlten Lösung des Aldehyds $95 \mathbf{b}(78 \mathrm{mg}, 0.20 \mathrm{mmol})$ in THF ( $2 \mathrm{~mL}$ ) wurde eine auf $-60^{\circ} \mathrm{C}$ vorgekühlte verdünnte Lösung von 2-Methyl1-propenylmagnesiumbromid $(0.40 \mathrm{~mL}$ einer $0.5 \mathrm{M} \mathrm{Lsg}$. in THF, $0.20 \mathrm{mmol})$ in THF $(1.6 \mathrm{~mL})$ per Transferkanüle „,in einem Schuss“ gegeben und die Reaktion nach 20 min durch Zugabe von ges. $\mathrm{NH}_{4} \mathrm{Cl}$-Lsg. $(1 \mathrm{~mL})$ beendet. Anschließend wurde die wässrige Phase mit MTBE $(3 \times 10 \mathrm{~mL})$ extrahiert, die vereinigten organischen Phasen mit ges. $\mathrm{NaCl}$-Lsg. ( $5 \mathrm{~mL}$ ) gewaschen und über $\mathrm{Na}_{2} \mathrm{SO}_{4}$ getrocknet. Säulenchromatographische Reinigung an Kieselgel (PE/MTBE 5:1, 0.3\% $i$-PrOH) lieferte die $\varepsilon$-Lactone 111a+b (48 mg, $0.13 \mathrm{mmol}, 62 \%, d r: 1: 1.3$ ) jeweils als farblose Öle. 
Analytische Daten von (+)-(5R,7R,1"R)-111(a):

Drehwert: $[\alpha]_{D}^{24}=+23.5^{\circ}\left(c 0.53, \mathrm{CHCl}_{3}\right)$.

$\mathbf{R}_{f}$-Wert: 0.29 (PE/EtOAc 4:1), 0.20 (PE/MTBE 4:1, 0.4\% $i$-PrOH).

${ }^{1} \mathbf{H}$ NMR $\left(600 \mathrm{MHz}, \mathrm{CDCl}_{3}\right): \delta 0.76 \mathrm{ppm}\left(\mathrm{s}, 3 \mathrm{H}, 5-\mathrm{CH}_{3}\right), 1.32-1.38\left(\mathrm{~m}, 1 \mathrm{H}, 4-\mathrm{H}_{\mathrm{a}}\right)$, $1.66\left(\mathrm{dd}, J=15.3,9.3 \mathrm{~Hz}, 1 \mathrm{H}, 6-\mathrm{H}_{\mathrm{a}}\right), 1.70-1.76\left(\mathrm{~m}, 2 \mathrm{H}, 3-\mathrm{H}_{\mathrm{a}}, 4-\mathrm{H}_{\mathrm{b}}\right), 1.73$ (d, $\left.J=1.1 \mathrm{~Hz}, 3 \mathrm{H}, 3^{\prime} \mathrm{a}-\mathrm{H}_{3}\right), 1.78\left(\mathrm{~d}, J=1.1 \mathrm{~Hz}, 3 \mathrm{H}, 3^{\prime} \mathrm{b}-\mathrm{H}_{3}\right), 1.82(\mathrm{dd}, J=15.3$, $\left.1.9 \mathrm{~Hz}, 1 \mathrm{H}, 6-\mathrm{H}_{\mathrm{b}}\right), 2.38-2.43\left(\mathrm{~m}, 1 \mathrm{H}, 3-\mathrm{H}_{\mathrm{b}}\right), 2.85\left(\mathrm{dd}, J=13.4,5.7 \mathrm{~Hz}, 1 \mathrm{H}, 2^{\prime \prime}-\mathrm{H}_{\mathrm{a}}\right.$ ), $2.96\left(\mathrm{dd}, J=13.4,7.7 \mathrm{~Hz}, 1 \mathrm{H}, 2^{\prime \prime}-\mathrm{H}_{\mathrm{b}}\right), 4.50\left(\mathrm{dd}, J=7.7,5.7 \mathrm{~Hz}, 1 \mathrm{H}, 1^{\prime \prime}-\mathrm{H}\right), 5.27$ (dsep, $\left.J=8.1,1.1 \mathrm{~Hz}, 1 \mathrm{H}, 1^{\prime}-\mathrm{H}\right), 5.36(\mathrm{t}, J=8.8 \mathrm{~Hz}, 1 \mathrm{H}, 7-\mathrm{H}), 6.99(\mathrm{~d}, J=7.6 \mathrm{~Hz}$, $2 \mathrm{H}, 2 \times \mathrm{Ph}-\mathrm{H}), 7.10-7.27$ (m, $8 \mathrm{H}, 8 \times \mathrm{Ph}-\mathrm{H})$.

${ }^{13} \mathbf{C}$ NMR $\left(126 \mathrm{MHz}, \mathrm{CDCl}_{3}\right): \delta 18.5 \mathrm{ppm}\left(\mathrm{C}-3\right.$ 'b), $25.5(\mathrm{C}-3$ 'a $), 26.3\left(5-\mathrm{CH}_{3}\right), 28.9$ (C-3), 32.6 (C-4), 46.4 (C-2"), 48.0 (C-6), 71.8 (C-7), 75.2 (C-5), 75.9 (C-1"), 124.0 (C-1'), 126.2 (C-Ph), 126.4 (2×C-Ph), 127.5 (C-Ph), 127.9 (2×C-Ph), $128.3(2 \times \mathrm{C}-\mathrm{Ph})$, $129.7(2 \times \mathrm{C}-\mathrm{Ph}), 135.4\left(\mathrm{C}-2^{\prime}\right), 138.1\left(\mathrm{C}_{i}-\mathrm{Ph}\right), 144.0\left(\mathrm{C}_{i}-\mathrm{Ph}\right), 175.4(\mathrm{C}=\mathrm{O})$.

IR (ATR): $\tilde{v} 3027 \mathrm{~cm}^{-1}, 2967,2934,2916,2853,1724(\mathrm{C}=\mathrm{O}), 1683(\mathrm{C}=\mathrm{C}), 1602$, 1453, 1439, 1378, 1334, 1295, 1253, 1174, 1102, 1058, 1009, 908, 760, 733, 699.

UV $\left(\mathrm{CH}_{3} \mathrm{CN}\right): \lambda_{\max }(\lg \varepsilon) 253 \mathrm{~nm}$ (2.7868), 259 (2.8061).

Analytische Daten von (+)-(5R,7S,1"R)-111(b):

Drehwert: $[\alpha]_{D}^{24}=+14.2^{\circ}\left(c 0.52, \mathrm{CHCl}_{3}\right)$.

$\mathbf{R}_{f}$-Wert: 0.26 (PE/EtOAc 4:1), 0.17 (PE/MTBE 4:1, $0.4 \% ~ i$-PrOH).

${ }^{1}$ H NMR $\left(600 \mathrm{MHz}, \mathrm{CDCl}_{3}\right): \delta 0.97 \mathrm{ppm}\left(\mathrm{s}, 3 \mathrm{H}, 5-\mathrm{CH}_{3}\right), 1.46$ (ddt, $J=14.3,7.4$, $\left.2.3 \mathrm{~Hz}, 1 \mathrm{H}, 4-\mathrm{H}_{\mathrm{a}}\right), 1.49-1.54\left(\mathrm{~m}, 1 \mathrm{H}, 6-\mathrm{H}_{\mathrm{a}}\right), 1.55\left(\mathrm{~d}, J=1.2 \mathrm{~Hz}, 3 \mathrm{H}, 3{ }^{\prime} \mathrm{a}-\mathrm{H}_{3}\right)$, 1.63-1.66 (m, $2 \mathrm{H}, 4-\mathrm{H}_{\mathrm{b}}, 6-\mathrm{H}_{\mathrm{b}}$ ), $1.65\left(\mathrm{~d}, J=1.2 \mathrm{~Hz}, 3 \mathrm{H}, 3{ }^{\prime} \mathrm{b}-\mathrm{H}_{3}\right), 2.28$ (ddd, $J=14.6$, 13.0, $\left.2.4 \mathrm{~Hz}, 1 \mathrm{H}, 3-\mathrm{H}_{\mathrm{a}}\right), 2.38\left(\mathrm{dd}, J=14.8,2.2 \mathrm{~Hz}, 1 \mathrm{H}, 3-\mathrm{H}_{\mathrm{b}}\right), 2.76(\mathrm{dd}, J=13.4$, $4.8 \mathrm{~Hz}, 1 \mathrm{H}, 2^{\prime \prime}-\mathrm{H}_{\mathrm{a}}$ ), 2.79 (dd, $J=13.4,8.5 \mathrm{~Hz}, 1 \mathrm{H}, 2^{\prime \prime}-\mathrm{H}_{\mathrm{b}}$ ), 4.55 (dd, $J=8.5,4.8 \mathrm{~Hz}$, $\left.1 \mathrm{H}, 1^{\prime \prime}-\mathrm{H}\right), 4.70(\mathrm{t}, J=9.1 \mathrm{~Hz}, 1 \mathrm{H}, 7-\mathrm{H}), 5.13\left(\mathrm{~d}, J=8.3 \mathrm{~Hz}, 1 \mathrm{H}, 1^{\prime}-\mathrm{H}\right), 7.09$ (d, $J=7.8 \mathrm{~Hz}, 2 \mathrm{H}, 2 \times \mathrm{Ph}-\mathrm{H}), 7.13-7.27$ (m, $8 \mathrm{H}, 8 \times \mathrm{Ph}-\mathrm{H})$. 
${ }^{13} \mathbf{C}$ NMR (126 MHz, $\left.\mathrm{CDCl}_{3}\right): \delta 18.4 \mathrm{ppm}(\mathrm{C}-3$ 'a $), 23.1\left(5-\mathrm{CH}_{3}\right), 25.5(\mathrm{C}-3$ 'b), 30.0 (C-3), 34.0 (C-4), 46.7 (C-2"), 47.0 (C-6), 72.8 (C-7), 75.8 (C-1"), 76.1 (C-5), 123.7

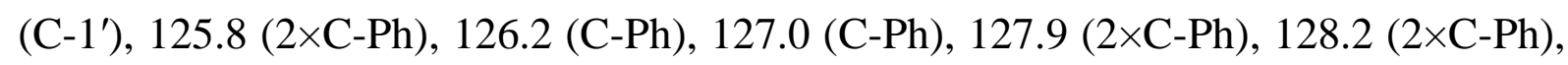
$129.8(2 \times \mathrm{C}-\mathrm{Ph}), 135.7\left(\mathrm{C}-2^{\prime}\right), 138.8\left(\mathrm{C}_{i}-\mathrm{Ph}\right), 145.2\left(\mathrm{C}_{i}-\mathrm{Ph}\right), 174.5(\mathrm{C}=\mathrm{O})$.

IR (ATR): $\tilde{v} 3061 \mathrm{~cm}^{-1}, 3028,2920,2856,1726(\mathrm{C}=\mathrm{O}), 1681,1603,1495,1452$, 1379, 1335, 1285, 1253, 1203, 1168, 1135, 1115, 1097, 1043, 978, 819, 758, 698.

UV $\left(\mathrm{CH}_{3} \mathrm{CN}\right): \lambda_{\max }(\lg \varepsilon) 253 \mathrm{~nm}$ (2.9107), 259 (2.9163).

MS (ESI, MeOH): $m / z(\%) 401.2(29)[\mathrm{M}+\mathrm{Na}]^{+}, 779.5(100)[2 \mathrm{M}+\mathrm{Na}]^{+}$.

$\mathbf{C}_{25} \mathbf{H}_{30} \mathbf{O}_{3}$ (378.50) ber.: 401.2087, gef.: $401.2086[\mathrm{M}+\mathrm{Na}]^{+}$(ESI-HRMS).

\section{$6.5 \quad(-)-\left(4 R, 6 R, 1^{\prime \prime} R\right)-4,8$-Dimethyl-4-(1,2-diphenylethoxy)-1-(3-furyl)- non-7-en-1-on (112a)}<smiles>CC(C)=CC(O)CC(C)(CCC(=O)c1ccco1)OC(Cc1ccccc1)(c1ccccc1)c1ccccc1</smiles>

$\mathrm{Zu}$ einer Lösung von 3-Bromfuran (103) $(6.0 \mu \mathrm{L}, 66 \mu \mathrm{mol})$ in trockenem THF (1 mL) wurde bei $-78{ }^{\circ} \mathrm{C} n$-BuLi $(0.24 \mathrm{~mL}$ einer $0.25 \mathrm{M} \mathrm{Lsg}$. in THF/Hexan 9:1, $60 \mu \mathrm{mol})$ getropft und 10 min bei dieser Temperatur gerührt. Nach Abkühlung der Lösung auf $-100{ }^{\circ} \mathrm{C}\left(\mathrm{EtOH} / \mathrm{N}_{2(1)}\right)$ wurde das $\varepsilon$-Lacton 111a $(12 \mathrm{mg}, 32 \mu \mathrm{mol})$ in THF $(0.4 \mathrm{~mL})$ über $30 \mathrm{~min}$ mit einer Spritzenpumpe zugetropft. Anschließend wurde die Reaktionslösung über $1 \mathrm{~h}$ auf $-60^{\circ} \mathrm{C}$ erwärmt und die Reaktion durch Zugabe von ges. $\mathrm{NH}_{4}$ Cl-Lsg. $(0.5 \mathrm{~mL})$ beendet. Die wässrige Phase wurde mit MTBE $(3 \times 5 \mathrm{~mL})$ extrahiert, die vereinigten organischen Phasen mit ges. NaCl-Lsg. gewaschen, über $\mathrm{Na}_{2} \mathrm{SO}_{4}$ getrocknet und das Lösungsmittel unter reduziertem Druck entfernt. Säulenchromatographische Reinigung an Kieselgel (PE/EtOAc 6:1) lieferte das Acylfuran 112a (5.8 mg, $13 \mu \mathrm{mol}, 42 \%)$ sowie das $\varepsilon$-Lacton 111a (5.8 mg, $15 \mu \mathrm{mol}$, 48\%) jeweils als farblose Öle. 
Drehwert: $[\alpha]_{D}^{24}=-2.8^{\circ}\left(c 1, \mathrm{CHCl}_{3}\right)$.

R -Wert: 0.23 (PE/EtOAc 4:1).

${ }^{1} \mathbf{H}$ NMR $\left(600 \mathrm{MHz}, \mathrm{CDCl}_{3}\right): \delta 1.01 \mathrm{ppm}\left(\mathrm{s}, 3 \mathrm{H}, 4-\mathrm{CH}_{3}\right), 1.19(\mathrm{dd}, J=14.5,1.6 \mathrm{~Hz}$, $\left.1 \mathrm{H}, 5-\mathrm{H}_{\mathrm{a}}\right), 1.61\left(\mathrm{ddd}, J=14.9,10.1,5.8 \mathrm{~Hz}, 1 \mathrm{H}, 3-\mathrm{H}_{\mathrm{a}}\right), 1.64(\mathrm{~d}, J=1.2 \mathrm{~Hz}, 3 \mathrm{H}$, $\left.9 \mathrm{a}-\mathrm{H}_{3}\right), 1.68\left(\mathrm{~d}, J=1.2 \mathrm{~Hz}, 3 \mathrm{H}, 9 \mathrm{~b}-\mathrm{H}_{3}\right), 1.75-1.82\left(\mathrm{~m}, 2 \mathrm{H}, 3-\mathrm{H}_{\mathrm{b}}, 5-\mathrm{H}_{\mathrm{b}}\right), 2.13$ (ddd, $\left.J=17.4,10.0,4.7 \mathrm{~Hz}, 1 \mathrm{H}, 2-\mathrm{H}_{\mathrm{a}}\right), 2.35\left(\mathrm{ddd}, J=17.4,10.0,5.7 \mathrm{~Hz}, 1 \mathrm{H}, 2-\mathrm{H}_{\mathrm{b}}\right), 2.90$ (dd, $J=13.5,5.7 \mathrm{~Hz}, 1 \mathrm{H}, 2^{\prime \prime}-\mathrm{H}_{\mathrm{a}}$ ), 2.98 (dd, $J=13.5,8.0 \mathrm{~Hz}, 1 \mathrm{H}, 2^{\prime \prime}-\mathrm{H}_{\mathrm{b}}$ ), 4.04 (s br., $1 \mathrm{H}, \mathrm{OH}), 4.61-4.66$ (m, $\left.2 \mathrm{H}, 1^{\prime \prime}-\mathrm{H}, 6-\mathrm{H}\right), 5.16$ (dsep, $\left.J=8.3,1.2 \mathrm{~Hz}, 1 \mathrm{H}, 7-\mathrm{H}\right), 6.49$ $\left(\mathrm{dd}, J=1.8,0.8 \mathrm{~Hz}, 1 \mathrm{H}, 4^{\prime}-\mathrm{H}\right), 7.07-7.13,7.16-7.25(2 \times \mathrm{m}, 10 \mathrm{H}, 2 \times \mathrm{Ph}), 7.30$ (t, $\left.J=1.6 \mathrm{~Hz}, 1 \mathrm{H}, 5^{\prime}-\mathrm{H}\right), 7.44\left(\mathrm{dd}, J=1.4,0.8 \mathrm{~Hz}, 1 \mathrm{H}, 2^{\prime}-\mathrm{H}\right)$.

${ }^{13}$ C NMR (126 MHz, $\left.\mathrm{CDCl}_{3}\right): \delta 18.2$ ppm (C-9a), $23.1\left(4-\mathrm{CH}_{3}\right), 25.7(\mathrm{C}-9 \mathrm{~b}), 32.7$ (C-3), 34.3 (C-2), 46.2 (C-5), 46.8 (C-2"), 65.4 (C-6), 76.3 (C-1"), 80.0 (C-4), 108.3 (C-4'), 126.4 (C-Ph), 126.5 (2×C-Ph), 127.1 (C-3'), 127.3 (C-Ph), 127.8 (C-7), 128.1

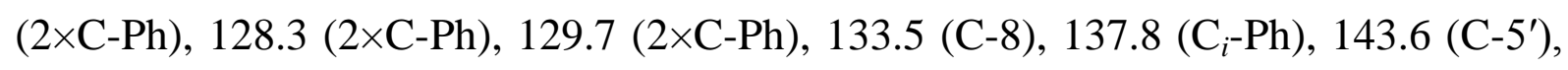
$143.7\left(\mathrm{C}_{i}-\mathrm{Ph}\right), 146.7\left(\mathrm{C}-2^{\prime}\right), 194.0(\mathrm{C}=\mathrm{O})$.

IR (ATR): $\tilde{v} 3474 \mathrm{~cm}^{-1}(\mathrm{OH}), 2921,1676(\mathrm{C}=\mathrm{O}), 1562,1509,1496,1453,1377,1154$, 1030, 935, 873, 811, 758, 699.

UV $\left(\mathrm{CH}_{3} \mathrm{CN}\right): \lambda_{\max }(\lg \varepsilon) 249$ (3.4469).

MS (ESI, MeOH): $m / z(\%) 469.2(100)[\mathrm{M}+\mathrm{Na}]^{+}, 915.4(94)[2 \mathrm{M}+\mathrm{Na}]^{+} ; 445.3$ (27) $[\mathrm{M}-\mathrm{H}]^{-}, 481.2(17)[\mathrm{M}+\mathrm{Cl}]^{-}, 491.3(100)\left[\mathrm{M}+\mathrm{HCO}_{2}\right]^{-}$.

$\mathrm{C}_{29} \mathrm{H}_{34} \mathrm{O}_{4}(446.58)$ ber.: 445.2384, gef.: $445.2379[\mathrm{M}-\mathrm{H}]^{-}$(ESI-HRMS). ber.: 469.2349, gef.: $469.2350[\mathrm{M}+\mathrm{Na}]^{+}$(ESI-HRMS). 


\section{$6.6(+)-(4 R, 6 S, 1 " R)-4,$,8 -Dimethyl-4-(1,2-diphenylethoxy)-1-(3-furyl)- non-7-en-1-on (112b)}

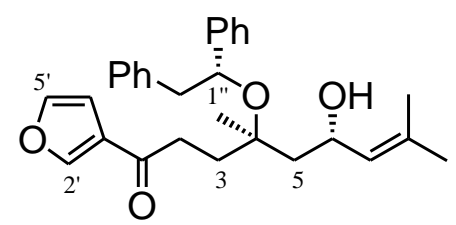

$\mathrm{Zu}$ einer Lösung von 3-Bromfuran $(\mathbf{1 0 3})(14 \mu \mathrm{L}, 140 \mu \mathrm{mol})$ in trockenem THF $(1.5 \mathrm{~mL})$ wurde bei $-78{ }^{\circ} \mathrm{C} n$-BuLi $(0.24 \mathrm{~mL}$ einer $0.25 \mathrm{M} \mathrm{Lsg}$. in THF/Hexan 9:1, $60 \mu \mathrm{mol})$ getropft und $10 \mathrm{~min}$ bei dieser Temperatur gerührt. Nach Abkühlung der Lösung auf $-100{ }^{\circ} \mathrm{C}\left(\mathrm{EtOH} / \mathrm{N}_{2(1)}\right)$ wurde das $\varepsilon$-Lacton 111b $(25 \mathrm{mg}, 66 \mu \mathrm{mol})$ in THF $(0.6 \mathrm{~mL})$ über $30 \mathrm{~min}$ mit einer Spritzenpumpe zugetropft. Anschließend wurde die Reaktionslösung über $1 \mathrm{~h}$ auf $-60^{\circ} \mathrm{C}$ erwärmt und die Reaktion durch Zugabe von ges. $\mathrm{NH}_{4}$ Cl-Lsg. $(0.5 \mathrm{~mL})$ beendet. Nach Die wässrige Phase wurde mit MTBE $(3 \times 5 \mathrm{~mL})$ extrahiert, die vereinigten organischen Phasen mit ges. NaCl-Lsg. gewaschen, über $\mathrm{Na}_{2} \mathrm{SO}_{4}$ getrocknet und das Lösungsmittel unter reduziertem Druck entfernt. Säulenchromatographische Reinigung an Kieselgel (PE/EtOAc 5:1) lieferte das Acylfuran 112b (13 mg, $29 \mu \mathrm{mol}, 44 \%)$ sowie das $\varepsilon$-Lacton 111b $(9.1 \mathrm{mg}, 24 \mu \mathrm{mol}$, $36 \%)$ jeweils als farblose Öle.

Drehwert: $[\alpha]_{D}^{24}=+0.5^{\circ}\left(c 1, \mathrm{CHCl}_{3}\right)$.

R-Wert: 0.17 (PE/EtOAc 4:1).

${ }^{1} \mathbf{H}$ NMR $\left(600 \mathrm{MHz}, \mathrm{CDCl}_{3}\right): \delta 0.88 \mathrm{ppm}\left(\mathrm{s}, 3 \mathrm{H}, 4-\mathrm{CH}_{3}\right), 1.46(\mathrm{dd}, J=14.7,2.9 \mathrm{~Hz}$, $\left.1 \mathrm{H}, 5-\mathrm{H}_{\mathrm{a}}\right), 1.66\left(\mathrm{~d}, J=1.0 \mathrm{~Hz}, 3 \mathrm{H}, 9 \mathrm{a}-\mathrm{H}_{3}\right), 1.70\left(\mathrm{~d}, J=1.0 \mathrm{~Hz}, 3 \mathrm{H}, 9 \mathrm{~b}-\mathrm{H}_{3}\right), 1.70$ (dd, $J=14.7,9.4 \mathrm{~Hz}, 1 \mathrm{H}, 5-\mathrm{H}_{\mathrm{b}}$ ), $1.78\left(\mathrm{ddd}, J=14.3,10.2,5.4 \mathrm{~Hz}, 1 \mathrm{H}, 3-\mathrm{H}_{\mathrm{a}}\right.$ ), 1.92 (ddd, $\left.J=14.3,10.1,5.5 \mathrm{~Hz}, 1 \mathrm{H}, 3-\mathrm{H}_{\mathrm{b}}\right), 2.46\left(\mathrm{ddd}, J=16.5,10.1,5.4 \mathrm{~Hz}, 1 \mathrm{H}, 2-\mathrm{H}_{\mathrm{a}}\right), 2.56$ (ddd, $\left.J=16.5,10.2,5.5 \mathrm{~Hz}, 1 \mathrm{H}, 2-\mathrm{H}_{\mathrm{b}}\right), 2.88\left(\mathrm{dd}, J=13.4,5.7 \mathrm{~Hz}, 1 \mathrm{H}, 2^{\prime \prime}-\mathrm{H}_{\mathrm{a}}\right), 2.99$ (dd, $\left.J=13.4,7.6 \mathrm{~Hz}, 1 \mathrm{H}, 2^{\prime \prime}-\mathrm{H}_{\mathrm{b}}\right), 4.59$ (dt, $\left.J=8.9,2.9 \mathrm{~Hz}, 1 \mathrm{H}, 6-\mathrm{H}\right), 4.70$ (dd, $\left.J=7.5,5.9 \mathrm{~Hz}, 1 \mathrm{H}, 1^{\prime \prime}-\mathrm{H}\right), 5.17$ (dsep, $\left.J=8.5,1.2 \mathrm{~Hz}, 1 \mathrm{H}, 7-\mathrm{H}\right), 6.63$ (d, $J=1.7 \mathrm{~Hz}$, $\left.1 \mathrm{H}, 4^{\prime}-\mathrm{H}\right), 7.04-7.07,7.15-7.30(2 \times \mathrm{m}, 10 \mathrm{H}, 2 \times \mathrm{Ph}), 7.37$ (t, $\left.J=1.6 \mathrm{~Hz}, 1 \mathrm{H}, 5^{\prime}-\mathrm{H}\right)$, $7.72\left(\mathrm{~s}, 1 \mathrm{H}, 2^{\prime}-\mathrm{H}\right)$. 
${ }^{13} \mathbf{C}$ NMR $\left(126 \mathrm{MHz}, \mathrm{CDCl}_{3}\right): \delta 18.3 \mathrm{ppm}(\mathrm{C}-9 \mathrm{a}), 24.4\left(4-\mathrm{CH}_{3}\right), 25.7$ (C-9b), 32.8 (C-2), 35.5 (C-3), 46.0 (C-5), 46.7 (C-2"), 65.5 (C-6), 76.1 (C-1"), 79.3 (C-4), 108.4 (C-4'), 126.2 (C-Ph), 126.3 (2×C-Ph), 127.1 (C-Ph), 127.3 (C-3'), 127.9 (2×C-Ph), $128.2(2 \times \mathrm{C}-\mathrm{Ph}), 128.3(\mathrm{C}-\mathrm{Ph}), 129.8(2 \times \mathrm{C}-\mathrm{Ph}), 133.7(\mathrm{C}-8), 138.1\left(\mathrm{C}_{i}-\mathrm{Ph}\right), 143.9$ $\left(\mathrm{C}-5^{\prime}\right), 144.4\left(\mathrm{C}_{i}-\mathrm{Ph}\right), 146.8\left(\mathrm{C}-2^{\prime}\right), 194.3(\mathrm{C}=\mathrm{O})$.

IR (ATR): $\tilde{v} 3459 \mathrm{~cm}^{-1}(\mathrm{OH}), 2922,1674(\mathrm{C}=\mathrm{O}), 1561,1509,1496,1453,1376,1154$, $1029,932,873,803,758,699$.

UV $\left(\mathrm{CH}_{3} \mathrm{CN}\right): \lambda_{\max }(\lg \varepsilon) 248 \mathrm{~nm}(3.3597)$.

MS (ESI, MeOH): $m / z(\%) 469.2(100)[\mathrm{M}+\mathrm{Na}]^{+}, 915.4$ (70) $[2 \mathrm{M}+\mathrm{Na}]^{+} ; 445.3$ (3)

$[\mathrm{M}-\mathrm{H}]^{-}, 481.2(17)[\mathrm{M}+\mathrm{Cl}]^{-}, 491.3(100)\left[\mathrm{M}+\mathrm{HCO}_{2}\right]^{-}$.

$\mathbf{C}_{29} \mathbf{H}_{34} \mathbf{O}_{4}(446.58)$

ber.: 469.2349 , gef.: $469.2347[\mathrm{M}+\mathrm{Na}]^{+}$(ESI-HRMS). 


\section{ANHANG}

\section{Röntgenstrukturdaten}

Kristalldaten

Formel

$\mathrm{C}_{29} \mathrm{H}_{30} \mathrm{~N}_{2} \mathrm{O}_{9}$

Molekulargewicht

550.55

Kristallsystem

monoklin

Raumgruppe

$\mathrm{P} 2{ }_{1}$

a, b, c [^]

6.8209(2), 27.0063(8), 7.7986(2)

$\alpha, \beta, \gamma\left[{ }^{\circ}\right]$

90, 111.561(1), 90

Volumen $V\left[\AA^{3}\right]$, Formeleinheiten $Z$

1336.04(7), 2

Kristallmaße [mm]

$0.18 \times 0.09 \times 0.07$

Dichte (ber.) $\rho\left[\mathrm{g} / \mathrm{cm}^{3}\right]$

1.369

Absorptionskoeffizient $\mu\left[\mathrm{mm}^{-1}\right]$

0.855

$\mathrm{F}(000)$

580

Messdaten

Temperatur (K)

100

Strahlung, $\lambda[\AA]$

$\mathrm{Cu}-\mathrm{K}_{\alpha}, 1.54178$

$\theta_{\min }, \theta_{\max }\left[{ }^{\circ}\right]$

$3.3,73.7$

Dataset

$-8: 8 ;-33: 33 ;-9: 7$

Tot., Uniq. Data, R(int)

42964, 5177, 0.034

Observed data $[\mathrm{I}>2.0 \sigma(\mathrm{I})]$

5109

Strukturverfeinerung

$\mathrm{N}_{\text {ref }}, \mathrm{N}_{\text {par }}$

5177,360

R, wR2, S

0.0396, 0.1173, 1.13

$\mathrm{W}=1 /\left[\sigma^{2}\left(\mathrm{~F}_{0}{ }^{2}\right)+(0.0493 \mathrm{P})^{2}+0.7950 \mathrm{P}\right]$ mit $\mathrm{P}=\left(\mathrm{F}_{0}{ }^{2}+2 \mathrm{~F}_{\mathrm{c}}{ }^{2}\right) / 3$

Max. and Av. Shift/Error

$0.00,0.00$

Flack x (konventionell)

$0.11(18)$

Parsons' Q-Werte Flack x

$-0.02(3)$

Min./Max. Restelektronendichte $\left[\mathrm{e} / \AA^{3}\right]$

$-0.30,0.35$ 


\section{Abkürzungen und Akronyme}

$\begin{array}{ll}\text { Ac } & \text { Acetyl } \\ \text { Äq } & \text { Äquivalent } \\ \text { aq. } & \text { wäßrig } \\ \text { atm } & \text { Atmosphäre } \\ \text { ADP } & \text { Adenosindiphosphat } \\ \text { AMP } & \text { Adenosinmonophosphat } \\ \text { ATP } & \text { Adenosintriphosphat } \\ \text { ber. } & \text { berechnet } \\ \text { brsm } & \text { based on recoverd starting material } \\ \text { BINOL } & 2,2 \text { '-Binaphthol } \\ \text { Bn } & \text { Benzyl } \\ \text { BuLi } & \text { Butyllithium } \\ c & \text { cyclo } \\ \text { CH }{ }_{2} \mathrm{Cl}{ }_{2} & \text { Dichlormethan } \\ \text { CDP } & \text { Cytidindiphophat } \\ \text { CMP } & \text { Cytidinmonophosphat } \\ \text { CoA } & \text { Coenzym A } \\ \text { COSY } & \text { correlated spectroscopy } \\ \text { CTP } & \text { Cytidintriphosphat } \\ \text { DBU } & 1,8 \text {-Diazabicyclo[5.4.0]undec-7-en } \\ \text { DC } & \text { Dünnschichtchromatographie } \\ \text { DMF } & \text { h,N-Dimethylformamid } \\ \text { DMSO } & \text { Dimethylsulfoxid } \\ \text { DuPHOS } & \text { vom Chemieunternehmen DuPont entwickeltes Phospholan } \\ e e & \text { enantiomeric excess } \\ \text { ESI } & \text { Elektrospray-Ionisation } \\ \text { Et } & \text { Ethyl } \\ \text { EtOH } & \text { Essigsäureethylester } \\ \text { EtOAc } & \text { gefunden } \\ \text { gef. } & \text { ges. } \\ \text { HMBC } & \end{array}$


HPLC

HRMS

HSQC

$\mathrm{Hz}$

$i$

$i$-Pr

IR

$m$

kat.

konz.

MCAR

$\mathrm{Me}$

$\mathrm{MeOH}$

$\mathrm{MgSO}_{4}$

MS

MTBE

MVA

$\mathrm{NaCl}$

$\mathrm{NaClO}_{2}$

$\mathrm{NADP}^{+}$

$\mathrm{Na}_{2} \mathrm{SO}_{4}$

$\mathrm{NaHCO}_{3}$

$\mathrm{NaH}_{2} \mathrm{PO}_{4}$

$\mathrm{NH}_{4} \mathrm{Cl}$

NMR

$o$

$\mathrm{OMe}$

$p$

p. a.

Pent

$\mathrm{Ph}$

$\mathrm{P}_{i}$

$\mathrm{PP}_{i}$

PS-DBU high performance (pressure) liquid chromatography

high resolution mass spectrometry

heteronuclear single-quantum correlation

Hertz

ipso

iso-Propyl

Infrarot-Spektroskopie

meta

katalytische Mengen

konzentriert

multi-component allylation reaction

Methyl

Methanol

Magnesiumsulfat

Molekularsieb / Massenspektrometrie

Methyl-tert-butylether

mevalunic acid

Natriumchlorid

Natriumchlorit

Nicotinamidadenindinucleotidphosphat

Natriumsulfat

Natriumhydrogencarbonat

Natriumdihydrogenphosphat

Ammoniumchlorid

nuclear magnetic resonance

ortho

Methoxy

para

für die Analyse geeigneter Reinheitsgrad (lat.:pro analysi)

$n$-Pentyl

Phenyl

inorganic phosphate

inorganic pyrophosphate

polymer-supported DBU 


$\begin{array}{ll}\mathrm{R}_{f} & \text { Retentionswert für DC } \\ t_{\mathrm{R}} & \text { Retentionszeit für HPLC } \\ \mathrm{RP} & \text { reversed-phase (Umkehrphase) } \\ \mathrm{RT} & \text { Umgebungstemperatur } \\ \mathrm{Std} . & \text { Standard } \\ \mathrm{Ti}(\mathrm{O} i \text {-Pr) })_{4} & \text { Titan(IV)-iso-propoxid } \\ \mathrm{TBAF} & \text { Tetrabutylammoniumfluorid } \\ t \text {-Bu } & \text { tert-Butyl } \\ t e r t & \text { tertiär } \\ \mathrm{TfOH} & \text { Trifluormethansulfonsäure } \\ \mathrm{THF} & \text { Tetrahydrofuran } \\ \mathrm{TMS} & \text { Trimethylsilyl } \\ \text { Tol } & \text { Tolyl } \\ \mathrm{Tr} & \text { Trityl bzw. Triphenylmethyl } \\ \mathrm{Ts} & \text { Tosyl ( } p \text {-Toluolsulfonyl) } \\ \mathrm{UV} & \text { Ultraviolett-Spektroskopie }\end{array}$




\section{Literatur}

[1] a) L. Ruzicka Experientia 1953, 9, 357-396. b) O. Wallach, Liebigs Ann. Chem. 1887, 129, 1-54.

[2] Reviews zur Terpenbiosynthese: a) E. Oldfield, F.-Y. Lin Angew. Chem. Int. Ed. 2012, 51, 1124-1137. b) T. Gräwert, M. Groll, F. Rohdich, A. Bacher, W. Eisenreich Cell. Mol. Life Sci. 2011, 68, 3797-3814. c) M. El-Sayed, R. Verpoorte Phytochem. Rev. 2007, 6, 277-305. d) V. M. Loyola-Vargas, R. M. Galaz-Ávalos, R. Kú-Cauich Phytochem. Rev. 2007, 6, 307-339.

[3] MEP-Biosyntheseweg: a) G. Flesch, M. Rohmer Eur. J. Biochem. 1988, 175, 405-411. b) M. Rohmer, M. Seemann, S. Horbach, S. Bringer-Meyer, H. Sahm J. Am. Chem. Soc. 1996, 118, 2564-2566. c) W.-C. Chang, Y. Xiao, H.-W. Liu, P. Liu Angew. Chem. Int. Ed. 2011, 50, 12304-12307.

[4] a) J. W. Cornforth, K. Clifford, R. Mallaby, G. T. Phillips J. Chem. Soc, Chem. Comm. 1971, 1599-1600. b) J. W. Cornforth, R. H. Cornforth, C. Donniger, G. Popják Proc. Roy. Soc., Lond. B 1966, 163, 452-514.

[5] C. Zdero, L. Lehmann, F. Bohlmann Phytochemistry 1991, 30, 1161-1163.

[6] Reviews zur asymmetrischen Allylierung: a) S. E. Denmark, J. Fu Chem. Rev. 2003, 103, 2763-2793. b) Y. Yamamoto, N.Asao Chem. Rev. 1993, 93, 2207-2293.

[7] a) AllylB(Ipc) $)_{2}$ : i) H. C. Brown, P. K. Jadhav J. Am. Chem. Soc. 1983, 105, 2092-2093. ii) H. C. Brown, K. S. Bhat J. Am. Chem. Soc. 1986, 108, 293-294. b) AllylB(2-Icr $)_{2}$ : H. C. Brown, R. S. Randad, K. S. Bhat, M. Zaidlewicz, U. S. Racherla J. Am. Chem. Soc. 1990, 112, 2389-2392. c) AllylB(4-Icr) 2 : H. C. Brown, P. K. Jadhav J. Org. Chem. 1984, 49, 4089-4091.

[8] Beispiele für Totalsynthesen mit Verwendung von Brown's AllylB(Ipc) $)_{2}$ :

a) G. Sabitha, S. S. S. Reddy, A. Raju, J. S. Yadav Synthesis 2011, 1279-1282.

b) B. Das, B. Veeranjaneyulu, P. Balasubramanyam, M. Srilatha Tetrahedron: Asymmetry 2010, 21, 2762-2767. c) T. Shigeyama, K. Katakawa, N. Kogure, M. Kitajima, H. Takayama Org. Lett. 2007, 9, 4069-4072. d) J. Zhang, Y. Li, W. Wang, X. She, X. Pan J. Org. Chem. 2006, 71, 2918-2921. 
[9] $\operatorname{AllylB(DIPT):~a)~W.~R.~Roush,~A.~E.~Walts,~L.~K.~Hoong~J.~Am.~Chem.~Soc.~}$ 1985, 107, 8186-8190. b) W. R. Roush, K. Ando, D. B. Powers, A. D. Palkowitz, R. L. Halterman J. Am. Chem. Soc. 1990, 112, 6339-6348.

[10] Beispiele für Totalsynthesen mit Verwendung von Roush's AllylB(DIPT):

a) S. Liu, Y. Fan, X. Peng, W. Wang, W. Hua, H. Akber, L. Liao Tetrahedron Lett. 2006, 47, 7681-7684. b) N. Kohyama, Y. Yamamoto Synlett 2001, 5, 694-696.

[11] a) R. Wada, K. Oisaki, M. Kanai, M. Shibasaki J. Am. Chem. Soc. 2004, 126, 8910-8911. b) M. Kanai, R. Wada, T. Shibuguchi, M. Shibasaki Pure Appl. Chem. 2008, 80, 1055-1062.

[12] S. Casolari, D. D`Addario, E. Tagliavini Org. Lett. 1999, 1, 1061-1063.

[13] a) K. M. Waltz, J. Gavenonis, P. J. Walsh Angew. Chem. Int. Ed. 2002, 41, 3697-3699. b) J. G. Kim, K. M. Waltz, I. F. Garcia, D. Kwiatkowski, P. J. Walsh J. Am. Chem. Soc. 2004, 126, 12580-12585. c) A. J. Wooten, J. G. Kim, P. J. Walsh Org. Lett. 2007, 9, 381-384.

[14] a) S. Kii, K. Maruoka Tetrahedron Lett. 2001, 42, 1935-1939. b) S. Kii, K. Maruoka Chirality 2003, 15, 68-70.

[15] Totalsynthesen mit Maruoka's asymmetrischer Allylierung von Aldehyden:

a) J. S. Yadav, A. Raju, K. Ravindar, B. V. S. Reddy Synthesis 2010, 797-802.

b) G. Sabitha, S. Nayak, M. Bhikshapathie, J. S. Yadav Tetrahedron Lett. 2009, 50, 5428-5429.

[16] a) M. Wadamoto, H. Yamamoto J. Am. Chem. Soc. 2005, 127, 14556-14557.

b) H. Yamamoto, M. Wadamoto Chem. Asian. J. 2007, 2, 692-698.

[17] a) Y. Okude, S. Hirano, T. Hiyama, H. Nozaki J. Am. Chem. Soc. 1977, 99, 3179-3181. b) T. Hiyama, Y. Okude, K. Kimura, H. Nozaki Bull. Chem. Soc. Jpn. 1982, 55, 561-568. c) K. Takai, K. Kimura, T. Kuroda, T. Hiyama, H. Nozaki Tetrahedron Lett. 1983, 24, 5281-5284. d) T. Hiyama, K. Kimura, H. Nozaki Tetrahedron Lett. 1981, 22, 1037-1040. e) K. Takai, T. Kuroda, S. Nakatsukasa, K. Oshima, H. Nozaki Tetrahedron Lett. 1985, 26, 5585-5588.

[18] a) katalytische NHK-Reaktion: A. Fürstner, N. Shi J. Am. Chem. Soc. 1996, 118, 12349-12357. b) Review: A. Fürstner Chem. Rev. 1999, 99, 991-1045. 
[19] a) J.-Y. Lee, J. J. Miller, S. S. Hamilton, M. S. Sigman Org. Lett. 2005, 7, 1837-1839. b) J. J. Miller, M. S. Sigman J. Am. Chem. Soc. 2007, 129, 2752-2753. c) K. C. Harper, M. S. Sigman Proc. Nat. Acad. Sci. 2011, 108, 2179-2183.

[20] a) S. Lou, P. N. Moquist, S. E. Schaus J. Am. Chem. Soc. 2006, 128, 12660-12661. b) D. S. Barnett, P. N. Moquist, S. E. Schaus Angew. Chem. Int. Ed. 2009, 48, 8679-8682. c) Anwendung auf cyclische Enone: D. F. Taber, D. A. Gerstenhaber, J. F. Berry J. Org. Chem. 2011, 76, 7614-7617.

[21] M. Nakamura, A. Hirai, M. Sogi, E. Nakamura J. Am. Chem. Soc. 1998, 120, 5846-5847.

[22] Beispiele für asymmetrische Allylierung von Aldehyden in der Totalsynthese:

i) Brown: a) J. S. Yadav, S. S. Mandal Tetrahedron Lett. 2011, 52, 5747-5749.

b) R. K. Prasad, K. Penchalaiah Tetrahedron: Asymmetry 2010, 21, 2853-2858.

c) T. K. Chakraborty, A. K. Chattopadhyay J. Org. Chem. 2008, 73, 3578-3581. ii) Roush: a) R. A. Fernandes, P. Kattanguru Tetrahedron Lett. 2011, 52, 1788-1790. b) S. Liu, Y. Fan, X. Peng, W. Wang, W. Hua, H. Akber, L. Liao Tetrahedron Lett. 2006, 47, 7681-7684. c) N. Kohyama, Y. Yamamoto Synlett 2001, 694-696.

[23] Beispiel für asymmetrische Allylierung von Enonen mit Nakamura-Reagenz:

a) F. Li, S. S. Tartakoff, S. L. Castle J. Am. Chem. Soc. 2009, 131, 6674-6675.

b) D. K. Nielson, L. L. Nielson, S. B. Jones, L .Toll, M. C. Asplund, S. L. Castle J. Org. Chem. 2009, 74, 1187-1199.

[24] a) A. Hosomi, H. Sakurai Tetrahedron Lett. 1976, 17, 1295-1298.

b) H. Sakurai Pure Appl. Chem. 1982, 54, 1-22. c) H. Sakurai, K. Sasaki, J. Hayashi, A. Hosomi J. Org. Chem. 1984, 49, 2808-2809.

[25] a) T. Mukaiyama, M. Ohshima, N. Miyoshi Chem. Lett. 1987, 16, 1121-1124.

b) A. Mekhalfia, I. E. Markó Tetrahedron Lett. 1991, 32, 4779-4782.

[26] J. R. Huckins, S. D. Rychnovsky J. Org. Chem. 2003, 68, 10135-10145.

[27] a) L. F. Tietze, L. Völkel, C. Wulff, B. Weigand, C. Bittner, P. McGrath, K. Johnson, M. Schäfer Chem. Eur. J. 2001, 7, 1304-1308. b) L. F. Tietze, K. Schiemann, C. Wegner, C. Wulff Chem. Eur. J. 1996, 2, 1164-1172. 
c) L. F. Tietze, C. Wegner, C. Wulff Synlett 1996, 471-472. d) L. F. Tietze, A. Dölle, K. Schiemann Angew. Chem. Int. Ed. 1992, 31, 1372-1373.

[28] a) L. F. Tietze, B. Weigand, L. Völkel, C. Bittner Chem. Eur. J. 2001, 7, 161-168. b) L. F. Tietze, K. Schiemann, C. Wegner, C. Wulff Chem. Eur. J. 1998, 4, 1862-1869. c) L. F. Tietze, C. Wegner, C. Wulff Eur. J. Org. Chem. 1998, 1639-1644. d) L. F. Tietze, K. Schiemann, C. Wegner J. Am. Chem. Soc. 1995, 117, 5851-5852.

[29] a) A. J. Birch J. Chem. Soc. 1944, 430-436. b) P. W. Rabideau, Z. Marcinow, Org. React. 1992, 42, 1-334. c) A. J. Birch Pure Appl. Chem. 1996, 68, $553-556$.

[30] Ammoniak-frei (LiDBBP): a) P. K. Freeman, L. L. Hutchinson J. Org. Chem. 1980, 45, 1924-1930. b) T. J. Donohoe, D. House J. Org. Chem. 2002, 67, 5015-5018.

[31] L. F. Tietze, S. Hölsken, J. Adrio, T. Kinzel, C. Wegner Synthesis 2004, 13, 2236-2239.

[32] C. C. Brazel Dissertation, Georg-August-Universität, Göttingen 2008.

[33] a) L. F. Tietze, T. Kinzel, T. Wolfram Chem. Eur. J. 2009, 15, 6199-6210;

b) L. F. Tietze, T. Kinzel, S. Schmatz J. Am. Chem. Soc. 2008, 130, 4386-4395.

c) L. F. Tietze, T. Kinzel, S. Schmatz J. Am. Chem. Soc. 2006, 128, 11483-11495.

[34] a) J. Robertson, A. Mallinger, B. Wu Org. Lett. 2010, 12, 2818-2821.

b) L. F. Tietze, S. Biller, T. Wolfram Synlett 2010, 14, 2130-2132.

[35] K. Manju, S. Trehan Chem. Comm. 1999, 1929-1930.

[36] Review zu Silicium in der organischen Chemie: I. Fleming, A. Barbero, D. Walter Chem. Rev. 1997, 97, 2063-2192.

[37] a) S. Itsuno, K. Ito, A. Hirao, S. Nakahama J. Chem. Soc., Chem. Comm. 1983, 469-470. b) E. J. Corey, R. K. Bakshi, S. J. Shibata J. Am. Chem. Soc. 1987, 109, 5551-5553. c) Y. H. Kim, D. H. Park, I. S. Byun, I. K. Yoon, C. S. Park J. Org. Chem. 1993, 58, 4511-4512. d) D. J. Mathre, A. S. Thompson, A. W. Douglas, K. Hoogsteen, J. D. Carroll, E. G. Corley, E. J. J. Grabowski J. Org. Chem. 1993, 58, 2880-2888. 
[38] Reviews zur Corey-Itsuno-Reduktion: a) E. J. Corey Pure Appl. Chem. 1990, 62, 1209-1216. b) S. Wallbaum, J. Martens Tetrahedron: Asymmetry 1992, 3, 1475-1504. c) G. B. Stone Tetrahedron: Asymmetry 1994, 5, 465-472.

[39] J. V. B. Kanth, M. Periasamy Tetrahedron 1993, 49, 5127-5132.

[40] a) D. B. Dess, J. C. Martin J. Org. Chem. 1983, 48, 4155-4156. b) D. B. Dess, J. C. Martin J. Am. Chem. Soc. 1991, 113, 7277-7287. c) R. E. Ireland, L. Liu J. Org. Chem. 1993, 58, 2899. d) R. K. Boeckman, Jr., P. Shao Org. Synth. 2000, 77, 141-146.

[41] a) B. S. Bal, W. E. Childers, H. W. Pinnick Tetrahedron 1981, 37, 2091-2096.

b) E. Dalcanale, F. Montanari J. Org. Chem. 1986, 51, 567-569. c) A. Raach, O. Reiser J. Prak. Chem. 2000, 342, 605-608.

[42] a) T. Kitaura, H. Endo, H. Nakamoto, M. Ishihara, T. Kawai, J. Nokami Flavour Fragr. J. 2004, 19, 221-224. b) Reaktionen mit Sulfoxoniumyliden: E. J. Corey, M. Chaykovsky J. Am. Chem. Soc. 1965, 87, 1353-1364.

[43] Allylische Oxidation: a) $\mathrm{SeO}_{2}$ : M. A. Umbreit, K. B. Sharpless J. Am. Chem. Soc. 1977, 99, 5526-5528. b) PDC: N. Chidambaram, S. Chandrasekaran J. Org. Chem. 1987, 52, 5048-5051. c) $M n(O A c)_{3}$ : T. K. M. Shing, Y.-Y. Yeung, P. L. Su Org. Lett. 2006, 8, 3149-3151. d) Review: M. B. Andrus, J. C. Lashley Tetrahedron 2002, 58, 845-866.

[44] Online-Tabellewerke von $p K_{a}$-Werten organischer Verbindungen:

a) F. G. Bordwell: http://www.chem.wisc.edu/areas/reich/pkatable/index.htm, Stand 1997. b) D. A. Evans: http://evans.harvard.edu/pdf/evans_pka_table.pdf, Stand 2005.

[45] L. Schröder, C. Schmitz, P. Bachert J. Mag. Res. 2005, 174, 68-77.

[46] a) S. Parsons, H. Flack In Acta Cryst., Band 60, 2004. b) S. Parsons, T. Wagner, O. Presly, P. A. Wood, R. I. Cooper J. Appl. Cryst. 2012, 45, 417-429.

[47] H. D. Flack Acta Cryst. A 1983, 39, 876-881.

[48] a) R. Lenz, S. V. Ley J. Chem. Soc., Perkin Trans. 1 1997, 3291-3292.

b) S. V. Ley, J. Norman, W. P. Griffith, S. P. Marsden Synthesis 1994, 639-666.

[49] http://www.chem.wisc.edu/areas/reich/handouts/nmr-c13/cdata.htm, Stand 10.02.2012. 
[50] a) K. Mori, N. Suzuki Liebigs Ann. Chem. 1990, 3, 287-292. b) I. Margaros, G. Vassilikogiannakis J. Org. Chem. 2007, 72, 4826-4831.

[51] D. D. Perrin, W. L. F. Amarego Purification of Laboratory Chemicals, $3^{\text {rd }}$ Ed., Pergamon Press, Oxford, 1988. 


\section{Danksagung}

$\mathrm{Zu}$ allererst möchte ich mich bei allen Mitarbeitern der analytischen Abteilungen des Instituts für Organische und Biomolekulare Chemie bedanken, ohne deren professionelle Arbeit die Anfertigung meiner Dissertation in dieser Form nicht möglich gewesen wäre. Für die Aufnahme der Massenspektren danke ich Frau Györgyi Sommer-Udvarnoki, Frau Gabriele Krökel und besonders Herrn Dr. Holm Frauendorf. Für die Aufnahme der Kernresonanzspektren möchte ich mich bei der NMR-Abteilung unseres Instituts bedanken, deren Mitarbeiter mir auch unter Einsatz geringster Substanzmengen immer traumhafte Spektren aufnahmen und jeden Extrawunsch erfüllten. Vielen Dank gilt daher Herrn Ulrich Leonhardt, Herrn Reinhard Machinek, Frau Christiane Siebert, Herrn Martin Weitemeyer und Frau Carola Zolke.

Des Weiteren danke ich Herrn Olaf Senge für die Unterstützung im Zusammenhang mit der HPLC sowie Herrn Frank Hambloch für die Überwachung unserer Sicherheit. Ein großes Dankeschön geht auch an Frau Evelyn Pfeil für das Messen zahlreicher UV- und IR-Spektren sowie der Drehwerte.

Großer Dank gilt Martina Pretor, die immer ein offenes Ohr und Ratschläge bei Computerproblemen jeglicher Art hatte, sowie „unserer“ Sekretärin Sabine Schacht für die effiziente Koordination unserer Anliegen. Ein Dank gebührt auch unseren Hausmeistern Frank Peters und Wolfram Matthies für ihre stete Hilfsbereitschaft. Den Mitarbeitern der Chemikalienausgabe, Herrn Holger Tucholla und Herrn Rupert Schrommek, danke ich für die vorbildliche Versorgung mit den benötigten Chemikalien und den Mitarbeitern der Werkstätten für die schnelle Reparatur jeglicher Laborgeräte.

Für das schnelle und sorgfältige Korrekturlesen und die konstruktiven Kritiken möchte ich mich ganz herzlich bei Stefan Jackenkroll, Galina Pestel und Simon Biller bedanken.

Der größte Dank gebührt jedoch meiner lieben Frau, und meiner Familie, die mir stets den nötigen Rückhalt gegeben haben. Danke für Eure Unterstützung und Liebe. Ohne Euch wäre ich heute bestimmt nicht der, der ich bin! 


\section{Lebenslauf}

\section{Persönliche Daten}

Name:

Thomas Wolfram

Geburtsdatum:

06.02.1984

Geburtsort:

Sangerhausen

Eltern:

Helmut Wolfram und Cordula Wolfram, geb. Thon

Staatsangehörigkeit:

deutsch

Familienstand:

verheiratet

\section{Schulausbildung und Wehrdienst}

1990-1994

1995-2003

28.06.2003

07/2003-03/2004

\section{Hochschulausbildung}

04/2004-09/2007

$11 / 2007-05 / 2008$

25.07.2008

$10 / 2008-03 / 2012$
Grundschule Heinrich Heine, Sangerhausen

Gymnasium Geschwister Scholl, Sangerhausen

Allgemeine Hochschulreife

Wehrdienst

Chemiestudium an der Georg-August Universität Göttingen

Diplomarbeit am Institut für Organische und Biomolekulare Chemie der Georg-August Universität Göttingen unter der Leitung von Prof. Dr. Dr. h.c. Lutz F. Tietze über das Thema: „Enantioselektive Synthese von tertiären Homoallylalkoholen ".

Diplomprüfung

Dissertation am Institut für Organische und Biomolekulare Chemie der Georg-August Universität Göttingen unter der Leitung von Prof. Dr. Dr. h.c. Lutz F. Tietze über das Thema „Asymmetrische Allylierung von prochiralen Ketonen und deren Anwendung in der Synthese von Pinnatolid und Furopinnatin“. 